\title{
Taming acoustic cavitation
}

David Fernández Rivas

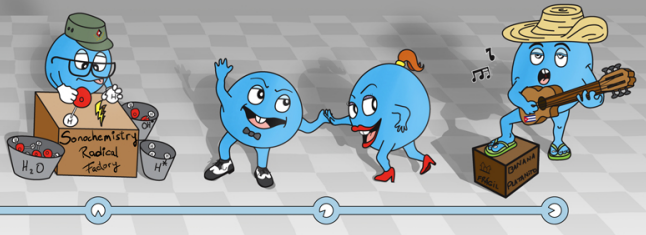




\section{Propositions}

accompanying the thesis of David Fernandez Rivas:

TAMING ACOUSTIC CAVITATION

1. Cavitating bubbles can behave in a "social fashion": they are "narcissist" as they like to collapse close to a wall reaching for their mirror image; when in a group or cluster, bubbles tend to shield each other, and if there is a close-by cluster and they have the right size or "age", all bubbles will rush to the "city-center" to have "fun" collapsing, jetting and emitting shock waves (screaming or making the roof shake) altogether making a mess of the floor after heavy-partying (erosion).

This thesis, Chapters 6, $7 \& 8$ and Thesis Cover.

2. Bubbles, as people, can behave in a strange way, deforming and collapsing sometimes alone, towards a wall, or even a neighbor bubble (quarreling). In these processes they can also split into other tinier bubbles (offspring), seeding the environment with bubbles that will oscillate in a subsequent acoustic cycle.

This thesis, Chapter 5 \& 6 and Thesis Cover.

3. Some bubbles have the ability to emit light (enligthened-gurus) by themselves while other produce radicals; sometimes the population of both type of bubbles overlap, and most of them (when in the right mood) are able to remove dirt; providing a good example of a collaborative society. At the same time, some less interesting bubbles just dissolve.

This thesis, Chapter 4 and Thesis Cover.

4. Happiness, fun, or even democracy cannot, or should not be imposed. One should try to facilitate its occurrence and wait for the best.

5. In scientific activities related to research, one must find the way to cope with those who are not that motivated or not willing to enjoy the process of learning or discovering new things; preventing them to spoil your curiosity and inspiration is crucial.

6. "Practicing Science" can keep you fit and sharp no matter how many hours we work or travel around the world.

Personal communication with a professor studying bubbles and cavitation.

7. If the weather conditions in southern Europe would be as "nice" as in northern Europe; (perhaps) there would be no "crisis". It would be very hard to spend hours enjoying outdoor activities (specially for bankers and politicians).

8. When analyzing scientific results, jumping to conclusions too soon can be dangerous; but it might be better to keep trying to explain a phenomenon than being defeated by its complexity.

9. Scientific activity holds the key to the future of Humanity; the moment it loses its human dimension, we humans will be doomed. 


\section{Taming acoustic cavitation}

MSc. Nucl. Eng. David Fernandez Rivas 


\section{Samenstelling promotiecommissie:}

Prof. dr. ir. R.G.H. Lammertink (voorzitter) prof. dr. J.G.E. Gardeniers (promotor)

Prof. dr. rer. nat. D. Lohse

prof. dr. M. Ashokkumar

prof. dr. K. Seshan

prof. dr. J.T.F. Keurentjes

dr. ir. N.E. Benes

dr. T.C. Bor
Universiteit Twente, TNW

Universiteit Twente, TNW

Universiteit Twente, TNW

University of Melbourne, Australia

Universiteit Twente, TNW

TU Eindhoven

Universiteit Twente, TNW

Universiteit Twente, CTW

\section{बTW' MESA+}

The work in this thesis was carried out at the Mesoscale Chemical Systems group of the Faculty of Science and Technology, and the MESA+ Institute for Nanotechnology, both at the University of Twente. This project is financially supported through STW project 07391 .

Nederlandse titel:

Het temmen van akoestische cavitatie.

\section{Publisher:}

David Fernández Rivas, Mesoscale Chemical Systems, University of Twente, P.O.Box 217, 7500AE Enschede, The Netherlands

http://mcs.tnw.utwente.nl

d.fernandezrivas@utwente.nl

dfrivascu@gmail.com

Cover design: Drawings and design, David Fernandez Rivas;

Coloring and composition, Sander Huisman.

Print: Gildeprint, Enschede

Cover legend:

(D) A bubble is born from a hosPITal.

(3) Sonoluminescent ("illuminated") bubble.

(B) "Narcissist" bubble attracted by its "image bubble".

(Q) "Super"-bubble able to clean and travel at high speed.

(Q) "Sonochemist" bubble splitting water molecules (making radicals).

(2) "Dancing" bubbles breaking the floor (erosion).

(2) Bubble playing a "Tres" making inaudible ultrasonic music (frequency higher than $20 \mathrm{kHz}$ ).

(C) David Fernandez Rivas, Enschede, The Netherlands, 2012.

No part of this work may be reproduced by print, photocopy or any other means without the permission in writing from the publisher.

ISBN: 978-90-365-3419-2, 


\title{
TAMING ACOUSTIC CAVITATION
}

\author{
PROEFSCHRIFT
}

ter verkrijging van

de graad van doctor aan de Universiteit Twente,

op gezag van de rector magnificus,

Prof. dr. H. Brinksma,

volgens besluit van het College voor Promoties

in het openbaar te verdedigen

op vrijdag 26 oktober 2012 om 12.45 uur

door

David Fernandez Rivas

geboren op 15 augustus 1981

te Havana, Cuba 
Dit proefschrift is goedgekeurd door de promotor:

Prof. dr. J.G.E. Gardeniers 
"Ciencia y libertad son llaves maestras que han abierto las puertas por donde entran los hombres a torrentes, enamorados del mundo venidero."

Science and freedom are the master keys that have opened the doors through which men pour in, falling in love with the upcoming world.

José Martí, "Respeto a Nuestra América”, La América, Nueva York, 1883.

“... qué es la música sino la compañera y guía del espíritu en su viaje por los espacios?”

... what is music but the companion and guide of the spirit in its journey through spaces?

José Martí, Amistad funesta, Nueva York, 1885.

"De todas las congojas de la vida premian los hijos buenos, y no tiene el mundo aplausos que valgan lo que el beso de vuelta en una frente pura..."

Of all grievances in life, the good sons ease us, and the world has no applauses worth a returned kiss on a pure forehead.

José Martí, “En casa”, Patria, Nueva York, 1892.

A mi tía Mabel, por su ejemplo, su confianza y amor infinitos.

A Gaby. 


\section{Contents}

1 Introduction 1

2 Merging Microfluidics and Sonochemistry: towards greener and more efficient micro-sono-reactors

3 Efficient Sonochemistry through Microbubbles Generated with Micromachined Surfaces 35

4 Sonoluminescence and Sonochemiluminescence from a Microreactor 47

5 Enhancing Acoustic Cavitation using Artificial Crevice Bubbles 67

6 Ultrasound Artificially Nucleated Bubbles and their Sonochemical Radical Production

7 Localized Removal of Layers of Metal, Polymer, or Biomaterial by Ultrasound Cavitation Bubbles

8 Erosion Evolution in Mono-crystalline Silicon Surfaces Caused by Acoustic Cavitation Bubbles

9 Closing Remarks and Outlook

Summary

Samenvatting

List of Publications during PhD

Acknowledgements

About the author... 
CONTENTS 


\section{1 \\ Introduction}

This chapter is intended to give the framework over which this Thesis was founded.

Each subsequent Chapter will contain its own introduction part. 


\subsection{Research Project Plan}

The Project 07391 of the Dutch Technology Foundation STW, with the title Efficient Sonochemical Microreactors, financed the research covered in this Thesis. The word "sonochemical" stands for the use of ultrasound to generate chemical and physical effects. The research goal was to significantly improve the energy efficiency of existing sonochemical reactors (by at least one order of magnitude). Its main objective was to design, develop and test energy efficient sonochemical microreactors. The plan was to miniaturize existing reactors or invent new ones, in order to gain full control over the appearance and collapse of bubbles (cavitation process), and its energetic aspects.

Industrial large scale sonochemical applications have been limited by the inefficiency of transferring electrical energy into a chemical product, leaving most of the applications to laboratory or small batch reactors. Converting electrical power into another output power is done with an ultrasonic transducer. During this process electrical and heat losses occur, and as the output power is transferred into a liquid phase where cavitating bubbles will appear, coupling losses exist as well. During cavitation, additional heat and energy losses may occur. More important, bubbles in a cluster shield each other diminishing the potential energy of collapse. Additionally, conventional ultrasound transducers have a diverging pressure-wave field that is also affected by the presence of bubbles.

Defining the sonochemical yield of a given process as the ratio of measured chemical effect and the energy injected to the system, typical values are on the order of $10^{-6}$. Most of the energy loss is known to be associated with bubble cavitation phenomena; the electrical transfer has been already optimized. During the project, attention was paid to a better understanding of the cavitation process, and the development of methods to direct the energy towards a given chemical desired effect. To achieve these goals it was necessary to improve the energy focussing effect of cavitating bubbles, a reduction of energy losses by secondary flows and interference effects, resonance, frequency optimization among others.

The main challenges in obtaining more efficient sonochemical reactors are based on the control and knowledge on:

- Location and appearance of bubbles,

- Reactor geometry and driving element features,

- Other parameters: liquid properties and volume, saturating gas, etc.,

- Specific application or desired goal; for example water treatment to reduce contaminants needs different ultrasonic conditions than the synthesis of nanoparticles. 
We present a concept for the generation of cavitation that can be adapted to many applications and combined with existing ultrasonic equipment. It is perhaps a solution for most sonochemical applications, in which controlled and tailored cavitation conditions are required; hence the title of this Thesis is Taming Acoustic Cavitation.

The path to obtain a working device to tame bubbles was not linear nor straightforward. To illustrate its complexity, in Figure 1.1 a Roadmap is outlined containing several phenomena and aspects that were addressed during the last five years. To understand in detail this Roadmap we point out that a corresponding introduction section will be given in each Chapter of this Thesis.

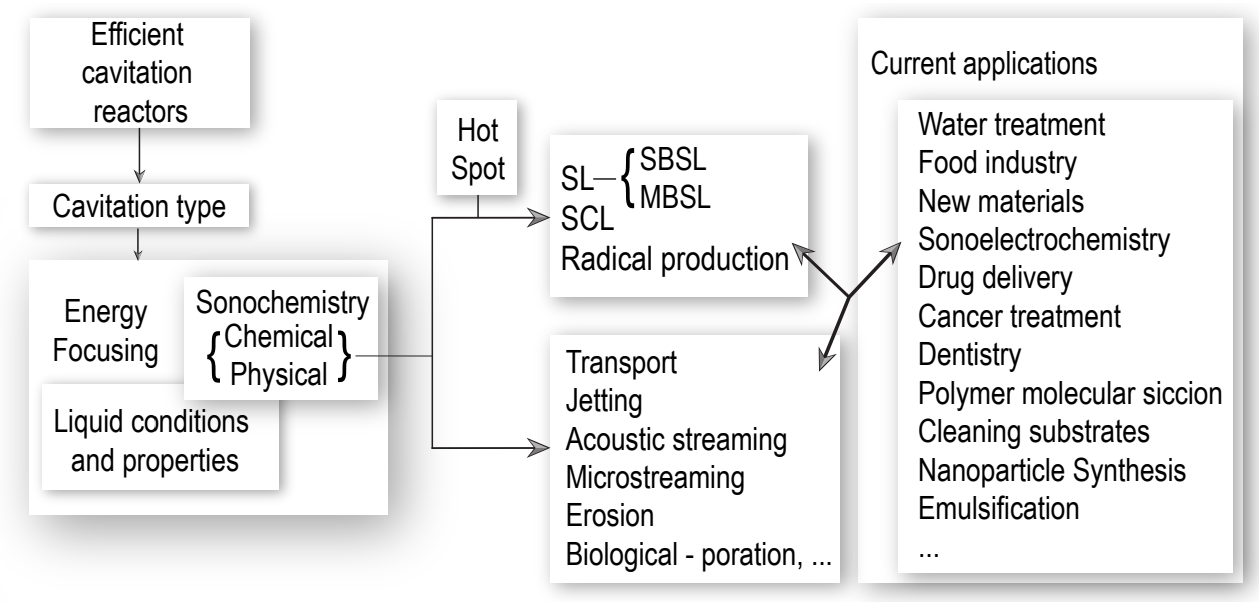

Figure 1.1: Roadmap to assist in understanding several aspects of sonochemistry and this Thesis.

To explain the Roadmap we start by defining that a bubble is a gas cavity surrounded by liquid. The relation between cavitation and chemistry can be understood in a simplified way by considering each oscillating bubble (growing and collapsing in a fast and adiabatic way) as individual high parameter reactors. The contents of the bubble will be heated and pressurized in a dramatic way, leading to an energy focusing effect.

The way the energy is distributed in the liquid and surrounding media will be determined mainly by the type of liquid and geometrical characteristics of the given system. Among the several explanations on why and how this Ultrasound (US) focusing effect takes place we make use of the Hot Spot Theory. The temperatures and pressures reached inside each cavitating bubble are high enough as to allow the formation of plasma and the lysis (sonolysis) of molecules, such as water molecule. Due to these unique conditions, a phenomenon known as Sonoluminescence (SL) takes place, which is light emitted from the interior of the bubble. Depending on the number of bubbles, it can be termed Single Bubble SL (SBSL) or Multi-Bubble 
SL (MBSL). If there are molecules dissolved in the liquid that can react with radicals produced in the bubbles and that emit light, it is termed Sonochemiluminescence (SCL).

The definition of what is Sonochemistry is expanded as we study in detail effects and applications useful in several fields of science and technology. Three main regions can be identified on which physicochemical phenomena occur: the gaseous interior of the bubble, the interphase between the interior of the bubble and the surrounding liquid, and the liquid bulk being sonicated. Among popular effects and applications of cavitation and sonochemistry we can mention the cleaning of teeth with ultrasonic dental tools [1], or surfaces such as eye contact lenses, and medical materials in US baths. Other non sonochemical applications of ultrasound techniques are the imaging of fetuses or the use of contrast agents for detecting heart problems. Not so known are the treatment of foodstuff like milk, drug delivery techniques that are less invasive for cancer treatment of tumors, water treatment for pharmaceutical purposes, mixing in complex reactions and others. The main feature found in this research field is that all the mentioned phenomena are connected in complex ways, and isolating one parameter without affecting another is virtually impossible.

The starting point of this research was planned to be the achievement of a better control over the generation or nucleation of bubbles. Once this control would be achieved, the bubbles could be set into cavitation with specific conditions. Our initial attempts to attain this control were by using a laser to heat a small volume of liquid in a micro-channel[2], afterwards by using a surface with small bubbles stabilized in artificial crevices or defects exposed to shockwaves (based on previous work [3]); but none yielded any measurable chemical effect. An alternative still under study is the use of monodisperse formation of stabilized microbubbles by flow-focusing devices [4] (see also Chapter 9).

The most successful reactor configuration studied was the continuous US irradiation of smooth surfaces with artificial crevices. The way it works is similar to what we can see in a beer glass, in which bubbles appear from certain places in the inner surface of the glass. These bubbles are nucleated from bubbles stabilized in natural defects. Artificial crevices micromachined in silicon substrates surfaces (which we call pits) can also stabilize a bubble when submerged in a liquid.

When applying continuous US to a small water volume, in which the substrates were submerged, we saw at relatively low pressure amplitudes interesting effects on particles dissolved in the liquid. Particles that accumulated on top of the oscillating bubble in the pit were ejected in a way resembling the eruption of a volcano. As the pressure amplitude increased, even more interesting things took place: the appearance of micro bubbles that travelled in strange trajectories that resembled some fireworks or scenes of "Star Wars" like in Fig. 1.2, left image.

Our imagination went even further in organizing pits in popular shapes, like mu- 
sical instruments (see Fig. 1.2, right image).
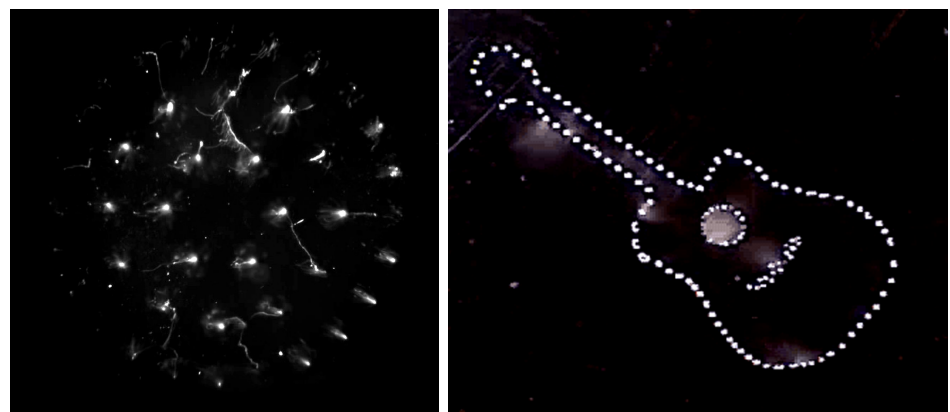

Figure 1.2: Left: Bubbles nucleating from a 42 pits array arranged in a concentric way and a guitar shape (Right). The white regions are the nucleated bubbles contrasting with the dark background.

\subsection{Thesis outline}

In Chapter 2 a review on the converging fields of microfluidics and sonochemistry is given with a "greener" and improved efficiency approach. Chapter 3 holds the first results in which an increased efficiency of a micro-sono-reactor was achieved with micromachined pits on its bottom surface. The SL and SCL emission from this micro-sono-reactor are described in Chapter 4 . A deeper study on the pinch-off which results in the enhanced acoustic cavitation provided by the presence of the pits is detailed in Chapter 5. In Chapter 6, the size distribution of bubbles ejected from the pits for different pressure amplitudes are correlated with sonochemical $\mathrm{OH}$ radical production. These results are also compared with numerical simulations of sonochemical conversion using the empirical bubble size distributions. A practical application in which the pits are useful is presented in Chapter 7, where the localized cleaning of several surfaces is demonstrated. Erosion effects on three different types of silicon substrates as a result of cavitating bubbles are studied in Chapter 8 , Chapter 9 serves as a general conclusion and future experimental ideas.

\section{References}

[1] R. Macedo, B. Verhaagen, D. Fernandez Rivas, J. G.E. Gardeniers, L. van der Sluis, P. Wesselink, and M. Versluis, "Sonochemical and visual characterization of cavitation generated by ultrasound in root canal models", Ultson. Sonochem to be submitted (2012).

[2] D. F. Rivas and J. G. E. Gardeniers, "On the resilience of PDMS microchannels after violent optical breakdown microbubble cavitation”, ASME Conference Proceedings 1939-1942 (2008).

[3] N. Bremond, M. Arora, C. D. Ohl, and D. Lohse, "Controlled multi-bubble surface cavitation", Phys. Rev. Lett. 96, 224501 (2006). 
[4] T. Segers, W. van Hoeve, D. Fernandez Rivas, J. G.E. Gardeniers, and M. Versluis, "Acoustic bubble sorting: manipulation of microbubbles by primary radiation forces", to be submitted to Lab-on-Chip (2012). 


\title{
2
}

\section{Merging Microfluidics and Sonochemistry: towards greener and more efficient micro-sono-reactors ${ }^{*}$}

\begin{abstract}
Microfluidics enable the manipulation of chemical reactions using very amounts of fluid, in channels with dimensions of tens to hundreds of micrometers. The immediate attraction of microfluidics lies in its greenness: use of small quantities of reagents and solvents, and hence less waste, precise control of reaction conditions, integration of functionality for process intensification, safer and often faster protocols, reliable scale-up, and possibility of performing multiphase reactions. Among the limitations found in microfluidics the facile formation of precipitating products should be highlighted, and in this context, the search for efficient mass and energy transfers is a must. Such limitations have been partially overcome with the aid of ultrasound in conventional flow systems, and can now be successfully used in microreactors, which provide new capabilities. Novel applications and a better understanding of the physical and chemical aspects of sonochemistry can certainly be achieved by combining microfluidics and ultrasound. We review this nascent area of research, paying attention to the latest developments and showing future directions, which benefit both from the existing microfluidic technology and sonochemistry itself.
\end{abstract}

${ }^{*}$ David Fernandez Rivas, Pedro Cintas, Han J.G.E. Gardeniers, Merging microfluidics and sonochemistry: towards greener and more efficient micro-sono-reactors, Feature Article in typesetting in Chem. Commun., DOI: 10.1039/C2CC33920J (2012).

†This article is dedicated to the late Dr. José González-García, a sonochemist colleague and friend. 


\subsection{Introduction and scope}

A few years ago chemists wondered whether traditional round-bottomed flasks would become replaced by microreactors [1]. Certainly, the use of microdevices now represents a realistic option that is becoming increasingly exploited in many laboratories. At first sight, the idea of using microfluidic technology in areas such as chemical synthesis seems rather unpractical and goes against the large-volume toolbox required by process research or the pharmaceutical industry. Motivations for the development of microfluidics have been, to a significant extent a triumph of imagination, by recognizing that the advantages of microelectronics could be extrapolated to chemical systems, cell biology, and many other fields [2-5]; and perhaps in part inspired by Feynman's remark: "There is plenty of room at the bottom" [6]. Counter-intuitively, miniaturized reactors have become instrumental in the kilogram-scale production of pharmaceuticals and chemicals involving potentially dangerous steps and more expensive production than for bulk chemicals and requiring additionally a higher research investment per unit weight produced [7-10].

Although continuous processes based on flow chemistry are also at their height, microreactors comprising small channel sizes and high surface area-to-volume ratios are usually more efficient by several orders of magnitude than their equivalent non-miniaturized counterparts [11]. In addition, there are fundamental differences between the physical properties of fluids in microchannels relative to those moving through large channels $[12-16]$. These physical features will not be detailed in this article, but we highlight a few distinctive characteristics associated with fluid streams in small dimensions. Notably in microfluidics the flow conditions are at low Reynolds numbers (i.e., laminar flow), which indicates that diffusion has a significant role rather than convection; the lack of inertial forces, and the existence of interfacial forces [17], and electro-osmotic flow [18], all of which are ultimately crucial, for instance, in enhanced mixing and particle separations.

There is an ongoing debate as to which dimensions the term microreactor should be employed [12]. Strictly speaking, the typical dimensions of fluid channels range from the sub-micrometer to the sub-millimeter level [19], with typical low flow rates not exceeding $\sim 1 \mathrm{~mL} \mathrm{~min}^{-1}$ ). Sometimes, small reactors claimed to involve microstructures have internal volumes of several milliliters, which are markedly different from chip-based reactors. Through this article, we will use the term microreactor and report channel dimensions whenever available. The puzzling terms nanoreactors, milli- or minireactors have been omitted for the sake of clarity. Microreactors can also be classified according to the operation mode, either continuous-flow or batch-type. While the former is usually preferred in chemical synthesis, other applications often require batch reactors.

The advent of ultrasound-driven chemical processes, in large or bench scale applications allows minimisation of problems like fouling and clogging, in continuous 
operation reactors. Thus, flow reactors benefit from mechanochemical effects such as efficient agitation and mixing after exposure to sound waves [20-23]. To avoid ambiguities with the use of ultrasound irradiation (to be distinguished from radiological activities), we prefer the term sonication.

Chemical and most physical effects of ultrasound arise from the cavitation collapse of bubbles in a sonicated liquid. Three regions of sonochemistry have been identified: bubble interior, bubble gas-liquid interface and liquid bulk [24-27]. Suslick and Price have defined three areas of chemical effects of ultrasound: homogeneous sonochemistry of liquids, heterogeneous sonochemistry of liquid-liquid or liquid-solid systems, and sonocatalysis overlaping the first two [20]. It is worthwhile to mention that the chemical effects of high-intensity ultrasound are driven by the appearance of bubbles and not due to the interaction of acoustic waves and matter at a molecular or atomic level.

Many sonochemical reactions can be rationalized assuming that volatile substances will undergo homolytic fragmentation and/or thermal excitation inside the bubbles (hot spot theory, which predicts temperatures and pressures in the range of $5000{ }^{\circ} \mathrm{C}$ and $\left.2000 \mathrm{~atm}\right)[20,24,28,29]$, thus producing radical or radical-ion intermediates that can react with species adsorbed in the gas-liquid interface or diffuse into the liquid bulk [30]. Some other known phenomena are related to the light emission from the interior of the bubbles known as sonoluminescence (SL), either from a single bubble (SBSL) or multibubbles (MBSL) [31-33]. These effects can be understood as particular examples of energy-focusing phenomena.

Bubble collapses are also accompanied by shock waves and shear forces which contribute to mixing and particle fragmentation when solids are present. A plethora of mechanical effects account for enhanced mass and energy transfer, resulting in acceleration, high conversions, rapid emulsification, polymer scission, and even supramolecular organization [24, 34-37]. The mechanical effects that can be pernicious sometimes (e.g. cavitation erosion caused by jetting), when controlled and well understood can be used in a productive way like in surface cleaning, drug delivery, cell poration, etc. [20, 38-40]. Figure 2.1 depicts an overview of the multiple phenomena induced by acoustic cavitation.

The combination of microstructures and sonication is a new and emerging area. Such a combination is technically feasible and often leads to synergic results. Although sonication has been largely employed to generate liquid flow and prevent the deposition of solids, it can do much more. Current research focuses on exploring sonochemical processes themselves with microfluidics. Under such conditions, cavitation collapse and associated phenomena like SL and radical production among others can be optimized and fine-tuned. One should bear in mind that numerous ultrasound-assisted reactions lack reproducibility, partly because cavitational energies depend on external parameters (frequency, intensity, dissolved gas, etc.) and 


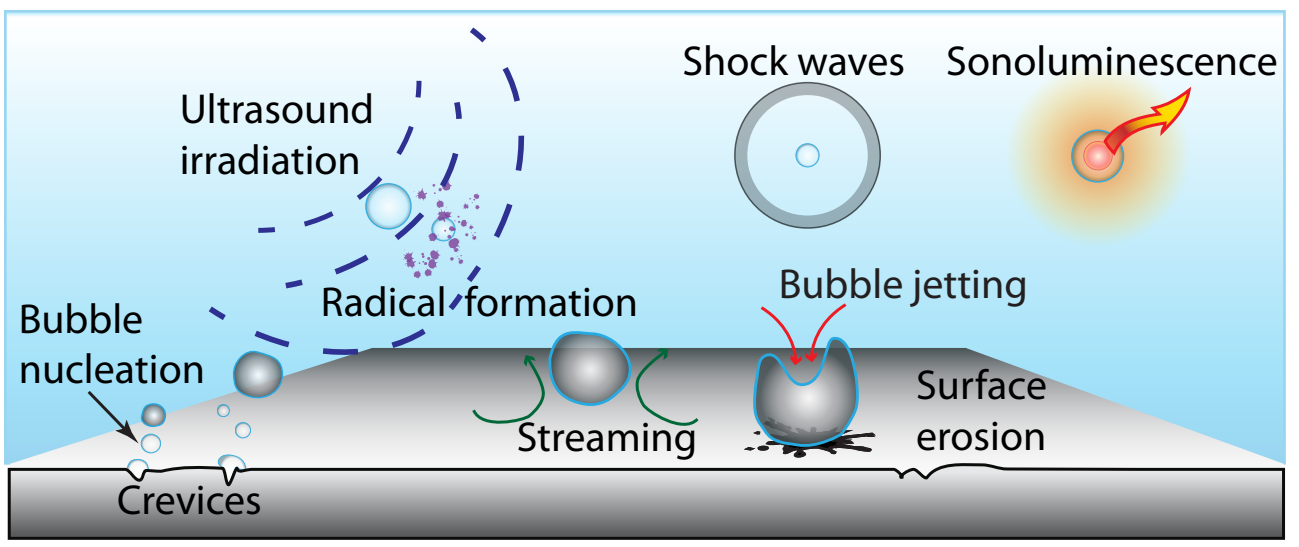

Figure 2.1: Schematic representation of the main effects of cavitation induced by ultrasound irradiation (sonication). Original artwork from the authors.

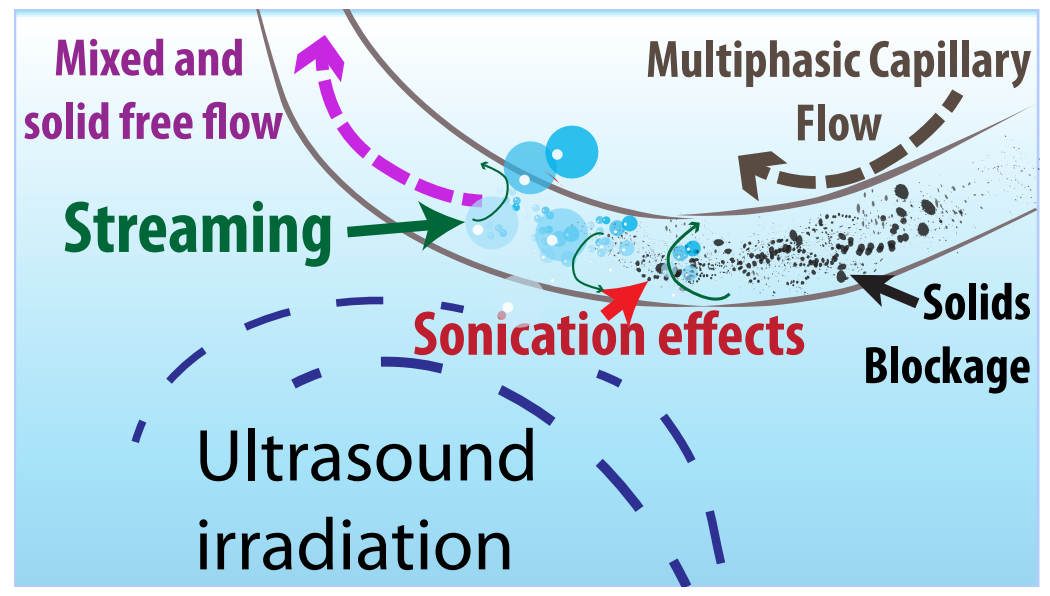

Figure 2.2: Schematic representation of sonication effects on a capillary through which a multiphasic (heterogeneous) mixture is pumped. The effects provided either by mixing or cavitation bubbles enhance transport and thereby reaction rates. Original artwork from the authors. 
solvent properties. Microfluidics devices are amenable for a precise control of temperature as well as frequency and pressure amplitudes combined with efficient mixing and short residence times. In addition, microfluidic systems allow the study of reactions at extreme conditions with reduced operation risks (explosive or run-away reactions, etc.). Moreover, large-volume applications only need scaling out or numbering up, which require a design in parallel of multiple reactors. Certain areas of chemical engineering can benefit already from the advantages of microfluidics, mainly those involving the production of pharmaceuticals or fine chemicals [7, 10, 11, 41-43].

Acoustic energy can be supplied in different forms, as transducers and piezoelectrical microdevices with different sizes and geometries are usually employed. These considerations are especially important regarding sound propagation and attenuation. Sound, unlike electromagnetic radiation, is not quantum energy and therefore parameters other than frequency may have a crucial influence; there is no direct relationship between energy and frequency. Power/intensity and above all fluid properties, will influence the outcome to a larger extent. This overview excludes an in-depth treatment of droplet-based surface acoustic waves (SAWs), which is also a versatile and promising technology, but not directly related to cavitational phenomena in liquids [44]. The approach of SAWs is based on shock waves with amplitudes of a few nanometers, which are generated by a piezoelectric inter-digital transducer (IDT). Liquid droplets placed on a surface are moved by the acoustic pressure. The technology is often called flat fluidics because no cover or slit is usually required [5]. SAWs and other ultrasonic applications, mainly in medicine, use frequencies in the $\mathrm{MHz}$ region, where a high intensity would be required to induce cavitation (in fact $\mathrm{MHz}$ frequencies are employed in medical imaging to avoid tissue damage) [21, 4547]. As we shall see later an important advantage of this miniaturized technology is the facile integration of piezoelectric actuators into microchips, which has been exploited in particle separations [48, 49]. SAWs have also found applications in synthetic chemistry [50].

It is convenient to highlight that some ultrasound-assisted microstructures do not necessarily involve cavitation, the core of sonochemistry -a fact not always mentioned explicitly in the literature. Therefore, interpretations of putative effects should be assessed with caution, ranging from efficient micromixing to temperature increases, reactor geometries and manufacturers themselves. It is difficult to cover all the current or potential applications of US cavitation in combination with microfluidics, among which we can mention: water treatment, fine chemical synthesis, food industry, sonoelectrochemistry, nanomaterials design and synthesis, drug delivery, dentistry, etc. As this article aims to point out, the combination of both fields can certainly yield new applications and improve the existing ones. 

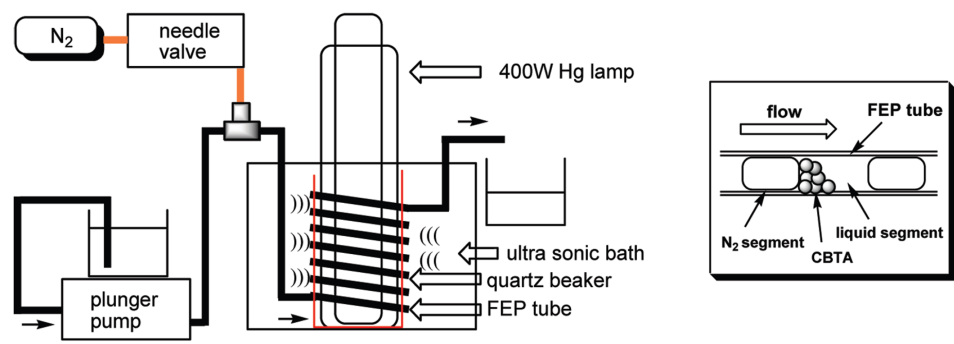

Figure 2.3: Clogging averted in a photochemical reaction with the aid of sonication. Inset: magnified microchannel made of fluorinated ethylene propylene (FEP) under flow. CTBA denotes a cyclobutane derivative, the photodimerization product of maleic anhydride. Reprinted with permission from ref. [55]. Copyright 2010 American Chemical Society.

\subsection{Managing heterogeneous reactions in microreactors}

As mentioned above, the use of acoustic radiation in capillary reactors and chipbased microstructures represents a solution for managing solids and overcomes the limitations of bridging and constriction; thereby enabling the use of high reagent concentrations. A simplified sketch in Figure 2.2 illustrates some of these effects.

Some reactors cannot be considered microreactors in the strict sense, as they use a capillary format of millimeter dimensions and pumps that give rise to flow rates in the $\mathrm{mL} \mathrm{min}^{-1}$ range. Such devices have become commercially available and have certainly revolutionized chemical synthesis. Herein, ultrasound can easily be applied by immersing the loops and tubing into an ultrasonic bath. Recent examples involve the oxidation of alcohols and aldehydes to carboxylic acids, as well as the Nef oxidation of nitroalkenes to carbonyls and acids, which can be conducted in a flow reactor using $\mathrm{KMnO}_{4}$ as oxidant and pulsed sonication.

Sonication prevents the blocking of the reactor and affords products in high yields and purity [51]. Buchwald and associates have employed the same strategy for Pdcatalyzed amination reactions in flow. The experimental set-up consisted of syringe pumps to deliver reagents to a microreactor made of polytetrafluoroethylene (PTFE) that was placed in an ultrasonic bath [52, 53]. Likewise, other synthetic transformations have been reported [54]. The versatility of these ultrasound-based modifications was further illustrated by the photodimerization of maleic anhydride [55]. The reaction solution was passed through a mm-sized tube, which was simultaneously irradiated with a high-pressure Hg lamp and an ultrasonic bath to prevent sedimentation and adhesion of solids (Figure 2.3.

Biphasic reactions in capillary reactors clearly benefit from emulsification effects provided by sonication, such as in the case of phase-transfer (PT) reactions [56]. Aqueous and organic fluids are fed by two separate lines and then mixed through a Tshaped connector. Sonication is supplied by a bath where a constant temperature can 


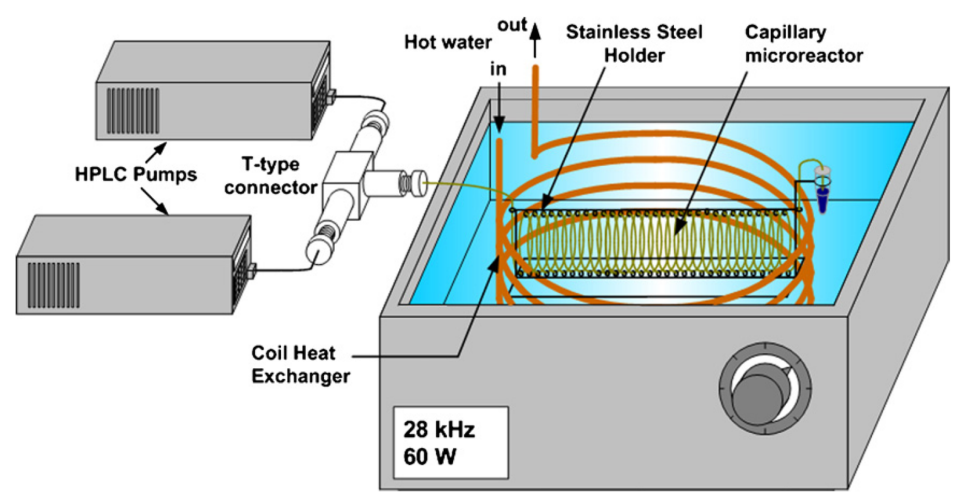

Figure 2.4: Schematic representation of an ultrasound-assisted capillary microreactor for the PT catalysis of benzyl chloride with sodium sulfide in the presence of tetrahexylammonium bromide. Reprinted with permission from ref. [56]. Copyright 2009 Elsevier B.V.

be maintained by an immersed coil-heat exchanger (Figure 2.4. In that study, sonication enhanced the conversion due to an efficient agitation that not only improved the mixing within one phase, but also helped in rupturing the interface between both phases, increasing the contact area. This purely mechanical effect could also be corroborated by a combination of mechanical stirring and sonication, which enhanced yields still further.

A model reaction involving the hydrolysis of p-nitrophenyl acetate was studied by Wirth et al. using segmented flow (rather than parallel flow) in a T-shaped geometry [41]. Fluid segments are stirred by internal vortices generated by the interaction of the liquid with the channel wall. By immersing the microchannel tubing (internal diameter ID $=300 \mu \mathrm{m}, \mathrm{L}=400 \mathrm{~mm}$ ) in an ultrasonic bath at $20 \mathrm{C}$, higher conversions with respect to conventional PT-catalysis and even under microwaves at $50{ }^{\circ} \mathrm{C}$ were observed. A slight enhancement could also be obtained by running the reaction with sonication plus a PT-catalyst.

The same hydrolysis has been re-investigated recently, using again segmented flow in a microstructured device (IDs ranging from 300 to $2400 \mu \mathrm{m}$; wall thicknesses of 400-800 $\mu \mathrm{m})$. Ultrasound was indirectly transmitted to the chemical system through a pressurized vessel filled with water (ca. $4.5 \mathrm{bar}$ ). The sonotrode (operating at $20 \mathrm{kHz}$ ) was placed axially at a distance of ca. $35 \mathrm{~mm}$ below the microdevice, which resulted in optimal intensity without causing material damage. The slug flow of the biphasic system could be monitored with a high-speed camera, showing that sonication leads to an apparently complete emulsification, thus increasing the interfacial area [57]. High yields (up to 86\%) were obtained with sonication, although they were also influenced by the channel diameter and material. Best results were achieved with PTFE (versus glass and PEEK = polyether ether ketone) tubing. Unfortunately, 
the set-up did not permit an accurate control of the temperature, especially for large IDs, for which the greatest yield enhancements were observed (with respect to silent conditions).

The above-mentioned design is based on a previous ultrasonic flow-through cell setup, consisting of a cylindrical steel jacket with an inner glass tube (ID $=2 \mathrm{~mm}$ ) for conducting the fluid, which was sonicated by a piezoelectric transducer $(24 \mathrm{kHz})$. Between the glass tube and the jacket, pressurized water (4.5-5.5 bar) was passed for sound transmission (Figure 2.5) [43]. Accordingly the apparatus is well suited for aseptic processing, often required in pharmaceutical and food industries. Application

(A)

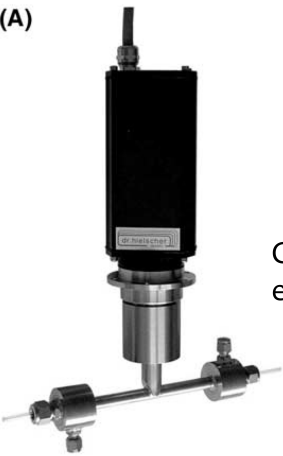

(B)

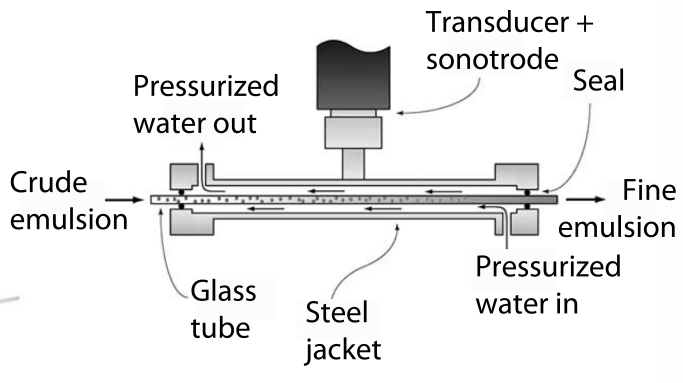

Figure 2.5: Ultrasonic flow cell (A: photograph, B: design) in which sound waves (24 kHz) are indirectly transmitted via pressurized water between the steel jacket and the glass tube containing the emulsion. Reproduced with permission from ref. [43]. Copyright 2006 Elsevier B.V.

to rapid formation of vegetable oil-in-water emulsions revealed the influence of both the acoustic power and residence times. Pre-emulsions had oil droplets ranging from 50 to $200 \mu \mathrm{m}$ while ultrasonic processing at $25 \mathrm{~W}$ gave rise to mean diameters of less than $1 \mu \mathrm{m}$, and eventually droplets of $5-10 \mu \mathrm{m}$. At $32 \mathrm{~W}$, no droplets were microscopically visible and the result was reproducible enough irrespective of the residence time (Figure 2.6). Below $17 \mathrm{~W}$ no appreciable emulsification could be observed.

The relationship between the applied pressure and cavitation conditions is very complex and will be affected by the ultrasound frequency and several other parameters (temperature, gas content, etc.) [58]. Intuitively one would expect that the larger the bubble before the collapse, the more energy is available for mechanical or chemical effects. The pressure applied influences the resonance between the acoustic field and the vibration frequency of the bubbles, whose radii are also a function of the pressure. The distance between bubbles and other objects will also have a significant effect on the types of collapses. A complete and thorough explanation is not trivial and has been discussed elsewhere [59-61]. 

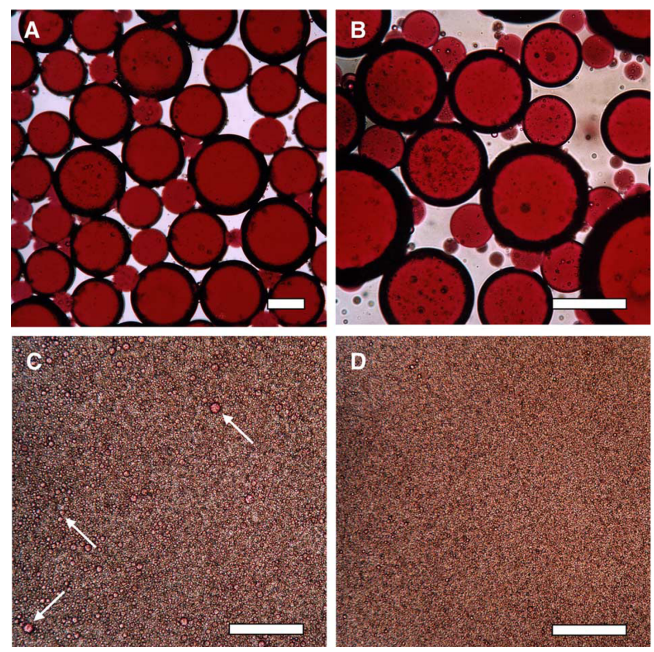

Figure 2.6: Light microscope micrographs of pre-mixed emulsions (A: 50- $\mu m$ droplets; B: 200- $\mu \mathrm{m}$ droplets) and sonicated emulsions at different acoustic power (C: $25 \mathrm{~W} ; \mathrm{D}: 32 \mathrm{~W}$ ) prepared with $20 \%$ (v/v) olive oil (stained with Fat Red 7B). Residence time: 13 s; bars denote $100 \mu \mathrm{m}$. Arrows in $C$ show single large droplets $(5-10 \mu \mathrm{m})$. Reproduced with permission from ref. [43]. Copyright 2006 Elsevier B.V.

\subsection{Miniaturized actuators}

Integration of small piezoelectric transducers into microfluidic channels can be accomplished through a variety of designs. As noted in the introductory remarks, an important application of standing waves in microfluidic chips is the continuous separation of mixed particle suspensions according to their properties in an acoustic field [62, 63].

The interaction of ultrasound with matter is a very complex one, in terms of the lengthscales and timescales involved, material properties, temperature and several other factors. For instance, a standing ultrasonic wave generates stationary pressure gradients, and if in a liquid medium, these gradients exert forces on particles with different density and compressibility; more details on the associated radiation and interparticle forces, heating, etc. are reported in literature [21, 50, 64].

A relatively simple device that showed no significant heating of the fluid was introduced by Yaralioglu et al. [65]. It consists of a series of transducers made of $\mathrm{ZnO}$ thin films (operating at ca. $450 \mathrm{MHz}$ ) placed at the bottom surface of a quartz substrate. The microfluidic channel, made of poly(dimethylsiloxane) (PDMS) is aligned to the transducers on the top surface. The transducers produce acoustic streaming perpendicular to the flow direction, thus enhancing mixing. Flow rates of 1-100 $\mu \mathrm{m} \mathrm{min}^{-1}$ were used for mixtures of phenolphthalein and $\mathrm{NaOH}$ solutions in ethanol. No bubble formation was observed at the MHz-region, which is in contrast 

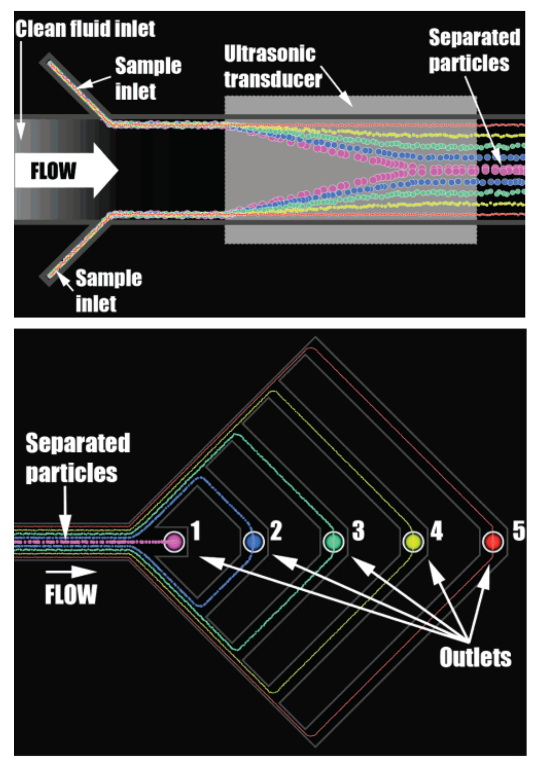

Figure 2.7: Scheme showing the action of acoustic standing waves on a particle suspension passing with laminar flow (top). Particles can be fractionated at the end of the channel (350 $\mu \mathrm{m}$ width) through consecutive outlets (bottom). Reproduced with permission from ref. [66]. Copyright 2007 American Chemical Society.

to the case when using power ultrasound.

The so-called free flow acoustophoresis enables for instance the fractionation of red cells, platelets and leukocytes using three outlets, provided that the density of the medium is altered with a salt [66]. The microtransducer worked at ca. $2 \mathrm{MHz}$ and the authors noted that the rise in temperature was less than $10{ }^{\circ} \mathrm{C}$ (Figure 2.7).

Acoustic microstreaming using air bubbles in microchannels is also an efficient protocol for micromixing [67]. The functioning of such a microdevice relies on a piezoelectric disk glued to a microchamber where air bubbles are trapped. The bubble oscillations induce a circulatory flow that results in convection and rapid mixing (Figure 2.8). In fact, sonication caused no motion of the liquid if air bubbles were not present, as was visualized with a red dye solution. The bubble-induced streaming is dependent on frequency for a given radius and, conversely on bubble radius for a given frequency. The microsystem worked at $5 \mathrm{kHz}$, below the ultrasonic threshold of ca. $20 \mathrm{kHz}$, although the low voltage also ensured a low power consumption (ca. 2 $\mathrm{mW}$ ), which makes the apparatus suitable for biological applications as inferred from a $E$. coli bacterial assay in red cells.

Another example of this type of actuation with intrinsic flow directionality without the need of using microchannels was presented by Marmottant, et.al. [68]. They used a range of frequencies to make different bubble sizes resonate $(20-200 \mathrm{kHz})$. 


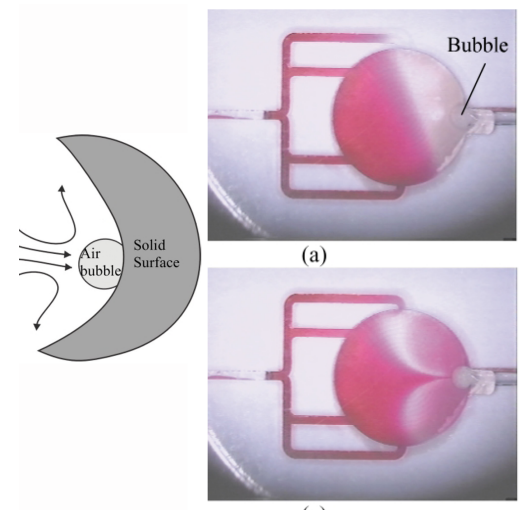

(c)

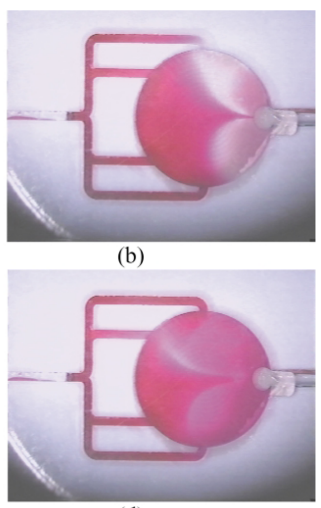

(d)

Figure 2.8: Action of acoustic microstreaming induced by an air bubble on a surface (left). Photographs showing microstreaming around a single air bubble in a chamber $(300 \mu \mathrm{m}$ depth, ID = $15 \mathrm{~mm})$ at 0, 15, 35 and $70 \mathrm{~s}(a, b, c$ and $d$, respectively). A piezoelectric disk attached to that chamber is not shown for clarity. Reproduced with permission from ref. [67]. Copyright 2002 The Royal Society of Chemistry.

Doublet arrays featuring pits with radii between 15 and $50 \mu \mathrm{m}$, combined with cylindrical bumps with a radius of $60 \mu \mathrm{m}$ and $30 \mu \mathrm{m}$ height. The distance between the centers of pit and bump within one doublet was of $95 \mu \mathrm{m}$, and the doublets interdistance $500 \mu \mathrm{m}$. The linear and small oscillations of the bubbles trapped in the pits resulted in vigorous agitation but no cavitation was observed in the liquid. The low ultrasonic amplitudes prevented heating the working fluid. Microfluidic transport was accomplished without pressure gradients and as stated above, without microchannels; making it a good alternative where clogging of channels in two-phase applications is undesired and unavoidable.

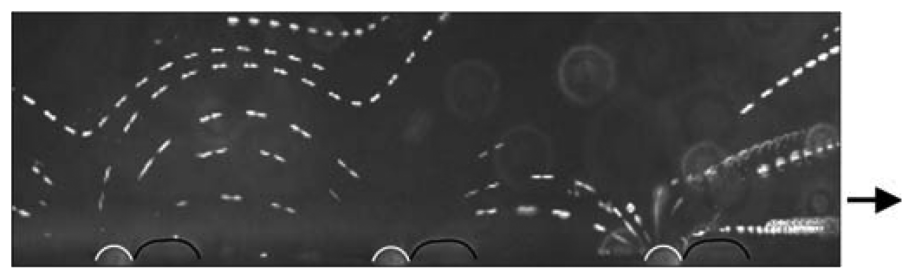

(i)

(ii)

(iii)

Figure 2.9: Side view of three bubble/bump doublets in experiment when bubbles are vibrating in response to ultrasound, with long-time exposure showing the transport of uorescent beads in the indicated direction (bubble radius $a=30 \mu \mathrm{m}$ ). Trajectories in the plane perdicular to the wall are visible. Reproduced with permission from ref. [68]. Copyright 2006 Cambridge University Press. 
A different design by Huang and associates, also harnesses single-bubble acoustic streaming by trapping an air bubble within a "horse-shoe" microstructure located between two laminar flows in a PDMS-based microchannel $(240-\mu \mathrm{m}$ width, $\mathrm{L}=1$ $\mathrm{cm})$. A piezoelectric transducer $(70 \mathrm{kHz})$ was bonded to the microfluidic device. Homogenized mixing could be attained within the millisecond range whereas no effect was observed in the absence of sonication [69]. Related experiments involve the use of oscillating bubbles excited at different $\mathrm{kHz}$-frequencies for particle trapping based on both size and density [70].

Iida et al. were able to observe microbubble related phenomena, synchronized with the ultrasonic wave by a stroboscopic technique [71]. Bubble oscillation, diffusion, the onset of cavitation, or microstreaming led to different spatial patterns depending on the input power. Figure 2.10 provides a schematic representation of the stroboscopic observation along with the geometry of a microreactor made of PDMS with a microchannel of $200 \mu \mathrm{m}$ width and $50 \mu \mathrm{m}$ depth. A larger microchannel (400 $\mu \mathrm{m}$ width and $200 \mu \mathrm{m}$ depth) was used for microstreaming observations. Sonication was provided via a miniaturized horn-type oscillator $(D=15 \mathrm{~mm}$, tip diameter $=10$ $\mathrm{mm}$ ) operating at ca. $60 \mathrm{kHz}$ in contact with the PDMS microreactor. Mechanical effects caused by microstreaming in the above mentioned reactor are also depicted in Figure 2.10. Yeast cells were added as tracer particles. On increasing the power density from 0.01 to $0.05 \mathrm{~W} \mathrm{~cm}^{-2}$, the streaming around the bubble increased to the half width of the channel. At $0.10 \mathrm{Wcm}^{-2}$ yeast cells moved violently, which may cause cell wall rupture. The direction of microstreaming was not apparently uniform and temporary changes in flow were observed, either toward or from the bubble.

The effect of surface-active solutes on the dynamic behavior of acoustic bubbles (coalescence, clustering, and fragmentation), at $60 \mathrm{kHz}$ under microconfinement, was reported by Lee et al. [72]. It was observed that bubbles coalesced instantly in water; however, in the presence of surface-active solutes (n-propanol and sodium dodecyl sulfate, SDS) the coalescence was hindered. The collective oscillation of a bubble cluster consisting of differently sized bubbles and images of bubbles emitting a fountain of microbubbles were reported.

Tandiono and collaborators [73] performed different studies in PDMS microchannels. A transducer was operated at $100 \mathrm{kHz}$ with pressure and vibration amplitudes of up to $20 \mathrm{bar}$ and $400 \mathrm{~nm}$ measured close to the covering top glass surface. The strong forcing resulted in the excitation of nonlinear surface waves when gas-liquid interfaces are present in the microfluidic channels. Nuclei leading to intense inertial cavitation were generated by the entrapment of gas pockets within the interfaces. Cavitation bubble clusters were recorded with high-speed photography at up to 250000 frames/s [73].

Whereas the above experiments illustrate analytical applications, the synthetic scenario is invariably constrained by solid-forming reactions. This concern has so 

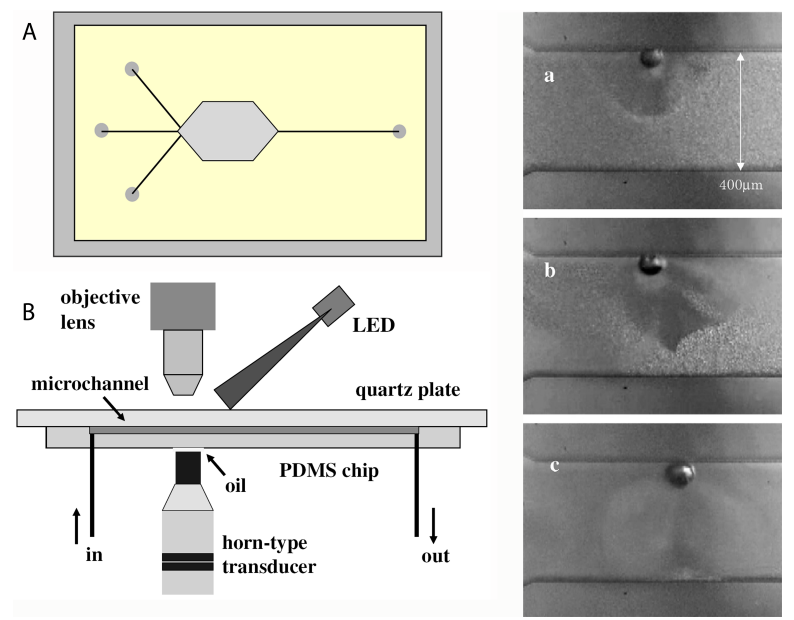

Figure 2.10: $A$ : The microreactor geometry is shown with bold lines and an hexagon representing the channels and pool, respectively. B: Stroboscopic observation of bubble motions in a micro-sono-reactor. Microstreaming induced by a bubble in a microchannel at different ultrasonic powers: (a) 0.01, (b) 0.05 and (c) $0.10 \mathrm{~W} \mathrm{cm-2.} \mathrm{Tracer} \mathrm{particles} \mathrm{are} \mathrm{yeast} \mathrm{cells.}$ Reproduced with permission from ref. [71]. Copyright 2007 Elsevier B.V.

far been solved by combining microfluidic devices with an ultrasonic bath, a strategy that is energetically inefficient with minuses associated with both temperature and power control. Home-made micro-sono-reactors have been developed by assembling PTFE plates $(70 \times 70 \mathrm{~mm}$, channel width $=600 \mu \mathrm{m})$ with a piezoelectric actuator (1-mm thickness), the latter being driven at different frequencies by a wave generator and amplification to an optimal power of $30 \mathrm{~W}$ (Figure 2.11] [74]. The resulting microreactor was used in Pd-catalyzed couplings of anilines and aryl halides, which led to precipitates $(\mathrm{NaCl}$ or $\mathrm{NaBr}$ ) in apolar solvents. Sonication at $50 \mathrm{kHz}$ prevented the microreactor from clogging (most particles were smaller than $20 \mu \mathrm{m}$ ), and excellent yields ( $>95 \%$ ) could be obtained within 60-90 s. Microchannel monitoring with an inverted microscope indicated that before sonication solid particles were present, which were subsequently broken up after sonication accompanied by bubble formation. This suggests that solvent degassing and cavitation are largely responsible for mechanical effects for that particular experiment.

\subsection{Sonochemical studies in micro-sono-reactors}

The conditions created after the implosion of cavitation bubbles in a liquid has been shown to enhance numerous chemical reactions [30, 42, 75,-77] and sometimes with the addition of particles to the liquid [78] or the modification of the surfaces of a sono-reactor [79]. Each cavitation bubble can be regarded as a microreactor possess- 


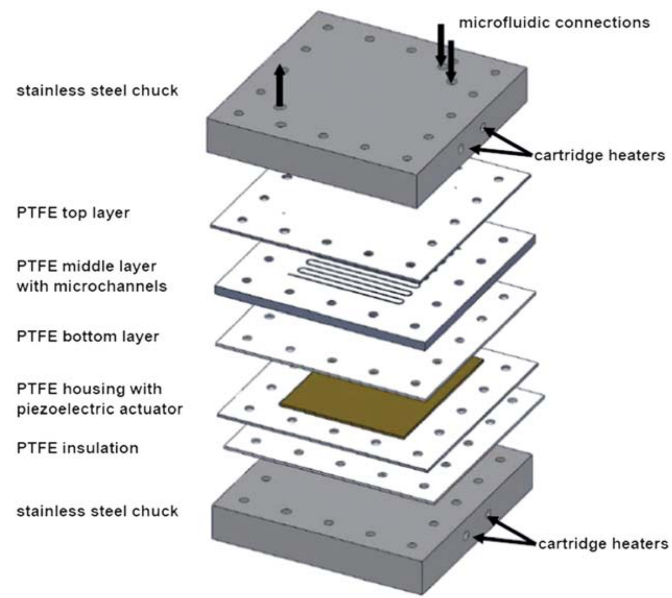

Figure 2.11: Representation of a microreactor assembly used for synthetic purposes, which includes three PTFE plates, the middle providing the microchannel structure. A piezoelectric actuator is integrated underneath the pile of PTFE plates and electrically insulated with a Teflon plate. The plates are compressed using two stainless steel chucks with holes for the insertion of cartridge heaters. Reproduced with permission from ref. [74]. Copyright 2011 The Royal Society of Chemistry.

ing a high-energy environment (as mentioned before, temperatures of $5000{ }^{0} \mathrm{C}$ and pressures of about $2000 \mathrm{~atm}$ ) that produces a plethora of excited chemical species [80, 81]. A single bubble collapsing in a reproducible way could be taken as the idealized reactor; like in experiments of SBSL [82-84]. Unfortunately, the amount of reagent inside a bubble is so tiny that these conditions have no practical value, though give theoretical support to SL and hot spot theoretical studies [28, 85]. The conditions for the appearance of bubbles and their subsequent cavitation are still not well understood, rendering the reproducibility of a given experiment a challenge defined by several physical-chemical factors. It has been concluded that in any case, each reaction-reactor system has to be optimized to fit a specific application [22, 86, -90].

Sonochemists regularly conduct experiments in vessels larger than the active zone where cavitation occurs (either using horns or baths). The problem of matching the ultrasonic field with the reactor dimensions can be partially solved with micromachined surfaces (modified surfaces or microchannels), as cavitation is then confined to small volumes and the nucleation sites for bubble appearance can be better controlled. Controlling cavitation events and a better understanding of such complex systems contributed to an increase in the energy efficiency of an ultrasonic microreactor (sono-micro-reactor) of the bath type [79].

To our knowledge, the first micro-sono-reactor study was presented by Iida and collaborators, who measured the production of $\mathrm{OH}^{\prime}$ radicals in microchannels under 
cavitation [91]. For comparative purposes, both the cavitational threshold and the efficiency to produce $\mathrm{OH}^{*}$ radicals by water sonolysis (see Equation 6.3) were evaluated in 1D- (a microchannel), 2D- (a thin layer) and 3D-microreactors, the latter being a glass tube (20-mm height and 25-mm diameter).

$$
\mathrm{H}_{2} \mathrm{O} \stackrel{\Delta \mathrm{H}=-5.1 \mathrm{eV}}{\rightarrow} \mathrm{OH}+\mathrm{H}^{\cdot}
$$

The corresponding components were tightly glued to a piezoelectric oscillator. The working frequencies were 162, 166 and $158 \mathrm{kHz}$ for 1D, 2D and 3D sonoreactors, respectively. The generation of $\mathrm{OH}^{\cdot}$ radicals produced by cavitation was monitored by their reaction with terephthalate anion giving rise to fluorescent hydroxyterephthalate, which represents a common chemical dosimeter in determining the cavitation threshold (Scheme 1). The detection limit of this dosimeter according to the experimental set-up of this study was $2.1 \times 10^{-13} \mathrm{~mol} \mathrm{~mL}^{-1}$ (equivalent to 1.2 $\times 10^{11}$ molecules $\mathrm{mL}^{-1}$ ).

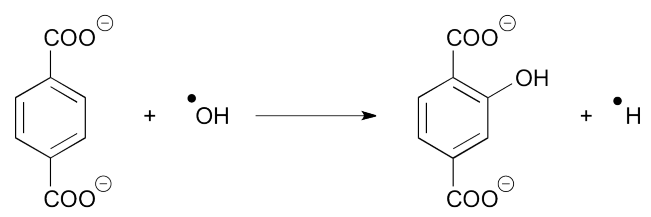

Scheme 1: Terephthalate anions as a trap for hydroxy radicals; the corresponding product can be monitored by fluorescence measurements.

The threshold value for the emergence of cavitation in the cases of 1D and 2D microreactors was ca. $0.7 \mathrm{~W} \mathrm{~cm}^{-2}$, much higher than that determined for the 3D case $\left(0.02 \mathrm{~W} \mathrm{~cm}^{-2}\right)$. The requirement of a high power density to induce cavitation in confined spaces can be rationalized assuming that bubble dynamics and hence the extension of collapse is constrained by the microspace. This could also account for the fact that cavitation in human tissues requires sound pressures considerably higher than those used in sonochemical reactors [92]. As expected, these experiments confirmed that the production rates of hydroxyterephthalate anions increased from 1D to $3 \mathrm{D}$ sono-reactors. However, taking into account the increase in reaction volumes (by 57 times from 2D to 3D), the efficiency of the three-dimensional reactor was poorer (reaction rate increased by 10 times).

Ohl's team, who had done work studying the effect of sonication of PDMS channels [73], looked in more detail at SL and sonochemiluminescence (SCL) in the same micro-system [93]. The bubbles form a planar/pancake shape in the microfluidics channels; and since the chemical reactions are closely confined to gas-liquid interfaces, they concluded that this spatial control of sonochemical reactions would prove useful in lab-on-a-chip devices (see Figure 2.12). The chemiluminescent reaction 
of luminol arises from ultrasonic generation of active radical species after cavitational collapse. Recombination of $\mathrm{OH}$ radicals produces hydrogen peroxide and this reagent is usually employed in laboratory demonstrations (Scheme 2).

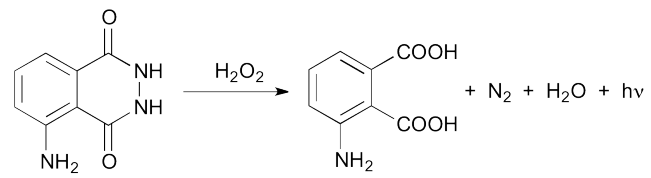

Scheme 2: Free radical species react with luminol producing bright sparks of light in the presence of sonic waves.

\subsubsection{Novel concept for micro-sono-reactors}

Further improvements on sonochemical efficiency in microreactors have been reported recently using pits micromachined on a silicon surface [79]. The application is based on the fundamental idea that, stable and monodisperse cavitation nuclei can be generated by trapping gas in such pits [94], which produce high yields at ultrasound powers that otherwise would not lead to a noticeable sonochemical effect. The main advantage of this method is that the location of cavitation occurrence is known a priori, which allows a detailed study of several relevant aspects of sonochemistry, such as SL, SCL and other effects such as erosion, cleaning action of bubbles, shock waves, jetting, streaming, etc. [38, 95, 96]; all of which have been difficult to address in the past, given the rather randomness of cavitating bubbles associated phenomena. Though the studies have been carried in the microscale, it has been proposed that this strategy of modifying the walls of a large scale sono-reactor, could lead to improvement in efficiency and versatility of already existent systems.

The experimental set-up is shown in Figure 2.13. The cylindrical pits $(30 \mu \mathrm{m}$ in diameter and depth of $10 \mu \mathrm{m}$ ) were obtained by using a plasma dry-etching machine and were arranged in three different configurations: single pits, two pits separated by a 1-mm distance, and three pits at the corners of an equilateral triangle with a side of $1 \mathrm{~mm}$. The pitted silicon chips were placed at the bottom of a glass vessel $(\mathrm{V}=300$ $\mu \mathrm{L}$ ) in which water sonolysis was investigated at $200 \mathrm{kHz}$ (an ultrasonic transducer was attached to the bottom of the chip).

Three different acoustic powers: $0.074 \mathrm{~W}$ (low), $0.182 \mathrm{~W}$ (medium) and 0.629 $\mathrm{W}$ (high); produce microbubbles ejected from the pits, though their trajectories and patterns are different as a result of Bjerknes forces and the geometry of the microsono-reactor as depicted in Figure 2.14. A single pit at low and medium powers ejects microbubbles along the axis of the pit that follow an ascending trajectory. This trend was also observed for two and three pits at low power. On increasing power, the microbubbles moved away from the symmetry axis of the pit and towards one 

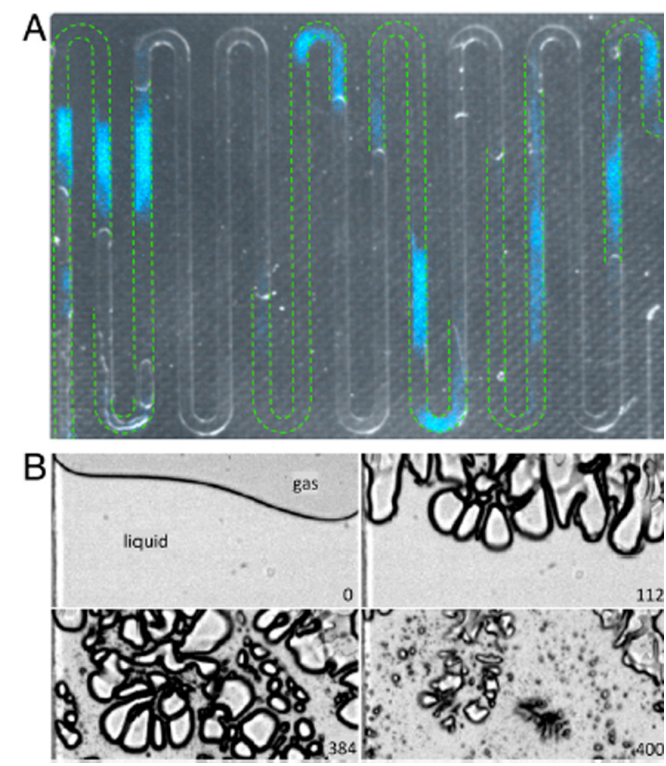

Figure 2.12: Luminol chemiluminescence from cavitation bubbles. (A) Composed picture of the channel geometry (gray background) showing the distribution of liquid and gas phases. The green dashed lines indicate the positions of liquid slugs just before the ultrasound is switched on. The bluish patches are taken with an intensified EMCCD camera and capture the chemiluminescence from the oxidation of luminol. (B) Sequence of images taken with a high-speed recording of the bubble distribution near a gas-liquid interface. The number in each frame is the time in microseconds after turning on the ultrasound. They show consecutively the initial gas-liquid interface leading to the severe surface instabilities, large bubble expansions, and finally the collapses. The interface was exposed to a harmonic driving at an amplitude of $230 \mathrm{~V}$ at its resonance frequency of $103.6 \mathrm{kHz}$. The width of each frame (channel) is $500 \mu \mathrm{m}$. Reproduced with permission from ref. [93]. Copyright 2007 the American Association for the Advancement of Science (AAAS).

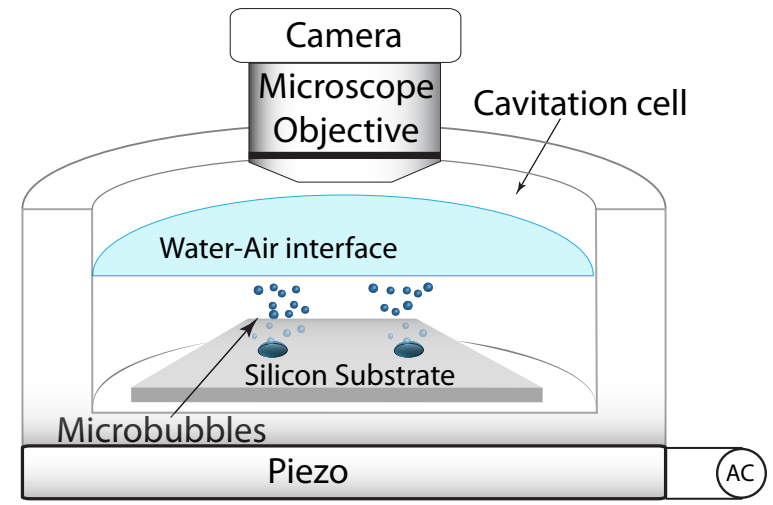

Figure 2.13: Experimental set-up showing the components to generate cavitation on pitcontaining micromachined silicon substrates. The piezo is controlled by an amplified wave generator signal. This system can provide top and side views of the cavitation events provided by the nucleation of bubbles from artificial crevices etched into a smooth silicon surface. 
another, thus creating a dense cloud around the midpoint of the pit arrangement.
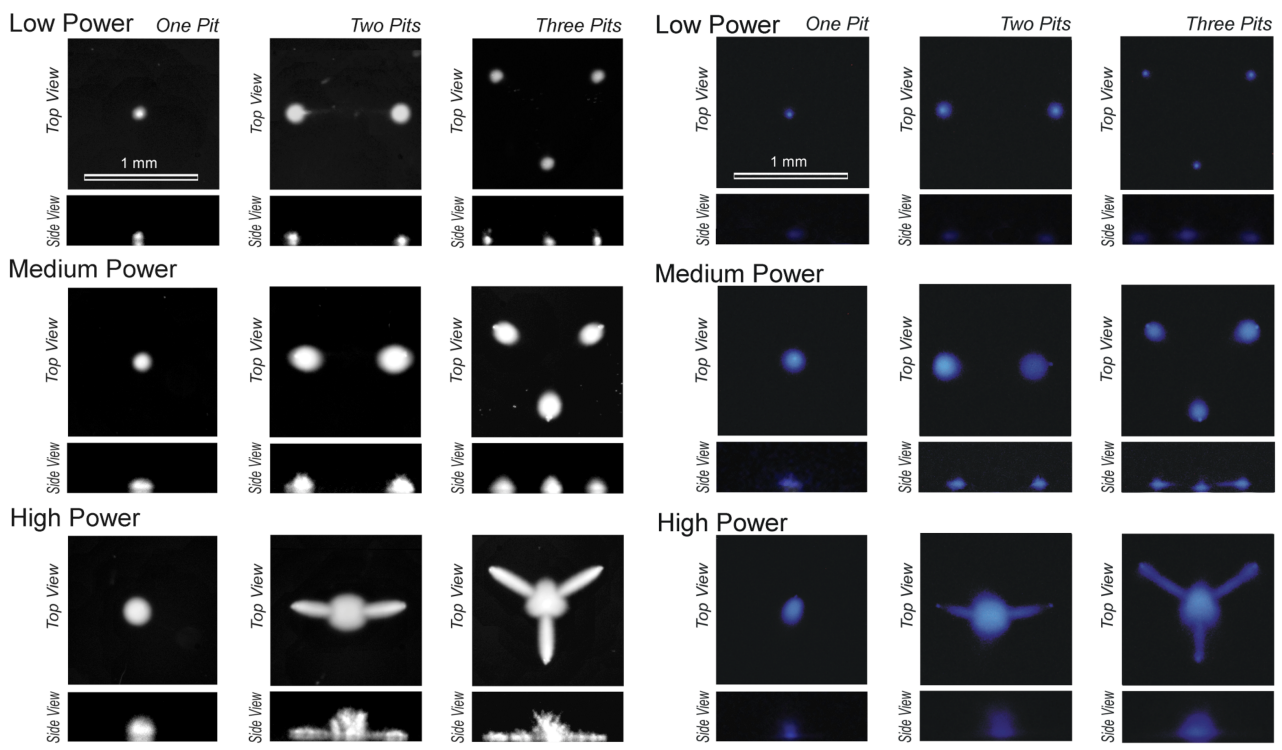

Figure 2.14: Bubble patterns generated from one, two and three pit arrangements for increasing acoustic powers: low $(0.074 \mathrm{~W})$, medium $(0.182 \mathrm{~W})$, and high $(0.629 \mathrm{~W})$. The left figure is the visible bubble streamers nucleated from the pits. The right figure comprises long exposure photographs in dark conditions of the same bubbles in luminol solution. Reproduced with permission from ref. [79]. Copyright 2010 Wiley-VCH Verlag GmbH \& Co. $K G a A$.

It is interesting to compare the power values of this type of reactor (see Table 2.1) with other reported values [91]. For the calorimetric values, the low power is comparable $\left(0.7 \mathrm{~W} \mathrm{~cm}^{-2}\right)$, whereas for larger power, this approach of gluing the piezo to the glass container seems to be able to inject more energy into the system $\left(2.59 \mathrm{~W} \mathrm{~cm}^{-2}\right)$.

Table 2.1: Electrical and calorimetric powers and their corresponding density values used in the micropits based sono-reactor.

\begin{tabular}{lcccc}
\hline Power & $\begin{array}{c}\mathrm{P}_{\text {Electr. }} \\
\mathrm{W}\end{array}$ & $\begin{array}{c}\mathrm{P}_{\text {Electr. }} / \text { Area } \\
\mathrm{Wcm}^{-2}\end{array}$ & $\begin{array}{c}\mathrm{P}_{\text {Calorim. }} \\
\mathrm{W}\end{array}$ & $\begin{array}{c}\mathrm{P}_{\text {Calorim. }} / \text { Area } \\
\mathrm{Wcm}^{-2}\end{array}$ \\
\hline Low & 0.074 & 0.047 & 3.32 & 2.11 \\
High & 0.629 & 0.400 & 12.7 & 8.09 \\
\hline
\end{tabular}

The evidence of the chemical activity provided by these cavitation bubbles were visualized by SCL of aqueous solutions of luminol (see Figure 2.14). Quantitative estimations of $\mathrm{OH} \cdot$ radicals by reaction with terephthalate anions clearly proved that 

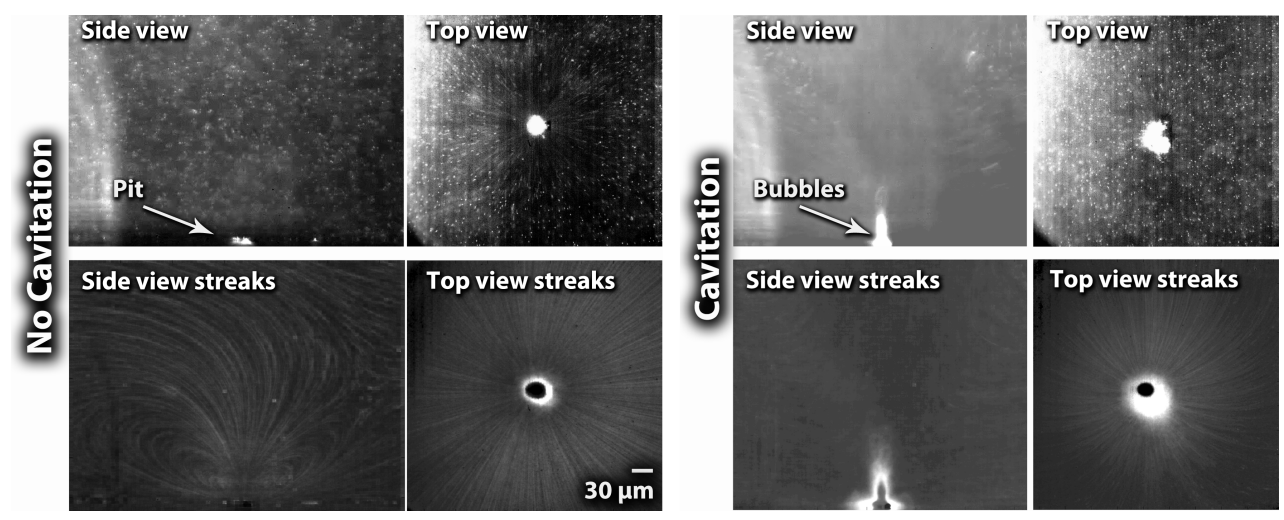

Figure 2.15: Streaming effects at $256 \mathrm{kHz}$ induced by the presence of a pit on a silicon surface when there are no bubbles ejected at $70 \mathrm{kPa}$ (left). The streak lines are changed and an increase in the liquid circulation takes place when bubbles are nucleated from the pit at $80 \mathrm{kPa}$ applied pressure (right).

the presence of pits enhanced significantly the radical formation rate, which was proportional to the number of pits and power setting. Though, the slope of the reaction rate curve shows a descending trend as the number of pits and power applied increased, which could be attributed to bubble-bubble and bubble cluster-cluster interactions [95, 96]. In contrast, non-pitted silicon substrates showed little evidence of ultrasonic effect. One could figure out that linear extrapolation to multiple-pit configurations in a sono-reactor will largely increase its efficiency. The nucleation of microbubble from the pits, and the subsequent cavitation of the gas seeded by the collapse of the initial nucleated bubbles, and perhaps other effects, as enhanced microstreaming, are certainly important [96].

How the presence of the pits can influence the micro-sono-reactor flow conditions is shown in Figure 2.15 (recorded at 250 frames per second), where the liquid was seeded with gold particles and the superposition of the recorded images allows the visualization of streaklines.

When amplitudes are kept lower than $80 \mathrm{kPa}$ for one pit, there is no cavitation and the bubble trapped on the pit induces the streaming. When bubbles nucleate from the pit at higher amplitudes, the streak lines are changed and an increase in the liquid circulation takes place.

More recent studies based on such a micro-sono-reactor also revealed that SL and SCL (assessed in aqueous luminol solutions) are directly related to the number of pits, which produce bubble clouds and cause a streaming effect. By monitoring $\mathrm{SL}$ in water and aqueous propanol, evidence of transient cavitation bubbles could be inferred from the latter. It is also noteworthy that SL and SCL in aqueous solutions gave different light intensities trends, which suggest that bubble populations respon- 
sible for light emission are different from the number of chemically-active bubbles [95].

The control of nucleation sites also allows to know with certain precission what bubble size distribution exists under given conditions, and a direct relation between the chemical effects can be obtained. This advantage has not only practical uses, but numerical modeling can be brought into play, in order to find optimum operation conditions that normally are not achievable with conventional sono-reactors. Among the other phenomena which can be recorded with such a reproducible and stable system are shockwaves, jetting and surface erosion [96].

Furthermore, the control of bubble nucleation sites represents an effective tool for the optimization of a given reaction; since different frequencies can be obtained with one system (contrary to most conventional sono-reactors which operate at a fixed frequency) and different amplitudes provide the desired bubble sizes and numbers. Figure 2.16 shows the same micro-sono-reactor system monitoring zones of $\mathrm{OH}$ radical production in the presence of luminol and SL signal at different frequencies.

Bubble cavitation close to surfaces is sometimes regarded as a negative effect (for instance, erosion of ship propellers). This phenomenon has been extensively used in conventional US cleaning baths. Traditionally when a surface needs to be cleaned, it is completely immersed in a liquid (e.g. water, acetone, ethanol or mixtures thereof) contained in a beaker and then sonicated. The cleaning effect takes place as bubbles nucleate randomly and cavitate close to the target surface. This means that sonication times can be quite long (more than $15 \mathrm{~min}$ ) and there is little control on the cleaning areas and rates. Recently, using the micro-sono-reactor with micromachined pits described above, it was demonstrated that a localized and faster cleaning of surfaces occurs with very small reaction volumes around $200 \mu \mathrm{L}$ [38]. The removal of metals, polymers and biomaterials (including cells and biofilms) may certainly find applications in surface science and biomedicine. Figure 2.17 shows facile removal of gold films deposited on glass slides when subjected to low- and high-power cavitation from three pits. Results were compared with conventional US bath cleaning effects, showing the advantages of small volumes and sonication times needed for the micro-sono-reactor (in less than $1 \mathrm{~min}$ ).

Since micromachined-pitted surfaces can be used to form microbubble clusters capable of producing mechanical effects (microstreaming, shock waves), other insightful mechanochemical reactions achieved recently in conventional sonochemical flasks could likewise be miniaturized. In this context, we highlight ultrasound-aided polymer scission, which is not a random process and takes place preferentially at weakened positions of the chain (mechanophores) [37]. Preliminary experiments have been conducted on ring-closing metathesis (RCM) of diallyl malonate using a Ru-based heterocyclic catalyst [97] in the same type of micro-sono-reactor with micropits [98]. As reported by Sijbesma and co-workers, a polymeric catalyst with a 

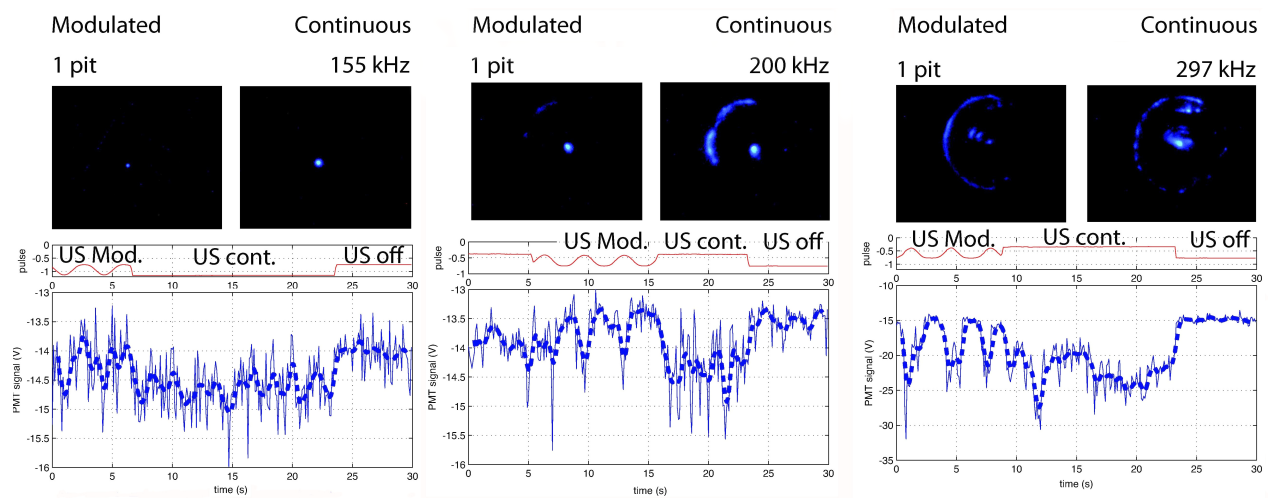

Figure 2.16: Top row shows areas of OH radical production via enhanced SCL (sonochemiluminescence of luminol). The bubbles were nucleated from one pit. For comparative purposes, continuous and modulated $(0.3 \mathrm{~Hz})$ oscillations are given for $S L$ signals at the bottom charts.

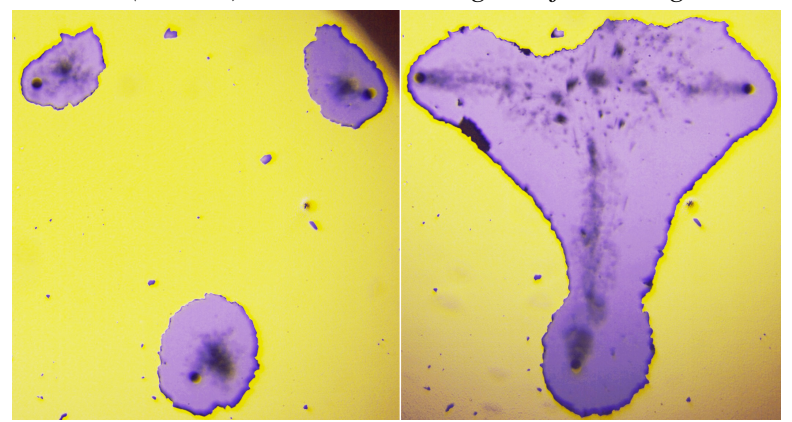

Figure 2.17: When a glass slide with deposited material is placed vertically close to three pits, cavitation bubbles emerge as the system is sonicated at low (left) and high power (right), removing gold (yellow) deposited on a glass slide. The pits are separated by a 1-mm distance. For more details see ref. [38].

molecular weight of ca. $34 \mathrm{kDa} \mathrm{mol}^{-1}$ should feel the forces generated by ultrasound, while this mechanical tension is negligible in the case of a control catalyst having small side chains (Scheme 3) [99].

Experiments using similar operation conditions as described above, using one and two pits, have been performed at different frequencies (ca. 170 and $230 \mathrm{kHz}$ ). Using the same concentration of reactants (malonate: $200 \mathrm{mM}$; catalyst: $0.2 \mathrm{mM}$ ), cavitation from the micromachined system (one pit, 0.04-005 W, V $=0.5 \mathrm{~mL}$ ) gave a conversion of only $1.14 \%$ after 30 -min sonication; which contrasts with the conventional sono-reactor (horn: $10-15 \mathrm{~W}, \mathrm{~V}=5 \mathrm{~mL}$ ) yielding ca. $30 \%$-product within the same time. Clearly this batch micro-sono-reactor needs to be improved, although greener approaches have already been formulated in microreactor technology as scaling-up is not usually required [100]. The latter could be accomplished either by increasing the 


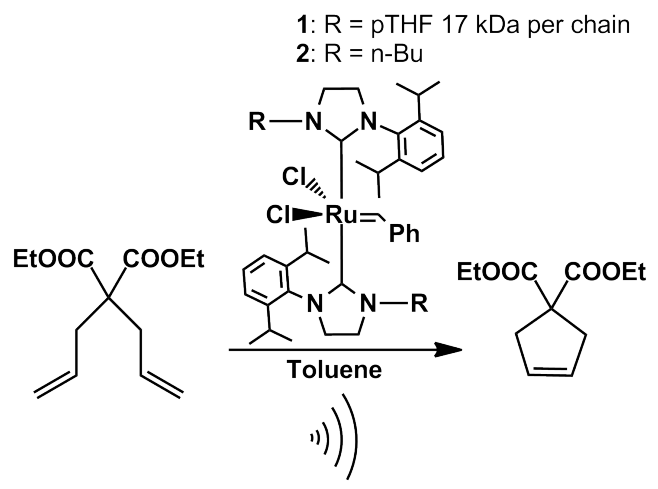

Scheme 3: Ring-closing metathesis reaction conducted with a polymer-based catalyst, which undergoes selective breaking under sonication.

number of pits or using a continuous protocol [101] (a 30-times recirculation would probably afford the conventional 30\%-conversion). Finally, and bearing in mind the above-mentioned results with micromachined silicon, it should not be difficult to find the optimal frequency-pressure conditions to increase efficiency, a difficult task with conventional sono-reators.

\subsection{Conclusions}

Microreactor technology has become a valuable and convenient approach to fulfil the criteria of green chemistry. While microfluidics was initially confined to analytical methods such as HPLC or capillary electrophoresis, miniaturization of continuousflow reactors offers a series of inherent pluses in chemical synthesis, high-throughput analysis, and the development of sensors that can be tailored for multiple applications. One cannot of course apply microfluidics to every conceivable chemical scenario, and numerous hurdles need to be considered. In order to improve both mass and energy transfer reactions, and avoiding formation of solid by-products, microreactors can now be equiped with piezoelectric transducers of miniature size as well. This innovative area is still in its infancy, though it should afford a new generation of lab-on-chip devices. The resulting micro-sono-reactors are capable of managing small reaction volumes in a reproducible and efficient way, whereas other parameters such as acoustic power, frequency, and sonication times can be fine-tuned. In addition, processes are scalable and enable flow recirculation. Last, but not least, sonochemical reactions conducted on micro-sono-reactors prove that there is a considerable enhancement of radical production and sonoluminescence, which can be harnessed in synthetic transformations in the near future. 


\subsection{Acknowledgements}

The authors would like to express their gratitude to A.G. Zijlstra for supplying particle flow videos and B. Verhagen with assistance in the streak images processing. R. Groote and R.T.M. Jakobs are acknowledged for their collaboration with RCM experiments. This research was supported by the Technology Foundation STW, Applied Science Division of NWO and the Technology Programme of the Ministry of Economic Affairs, The Netherlands.

\section{References}

[1] K. Geyer, J. D. C. Codée, and P. H. Seeberger, "Microreactors as tools for synthetic chemists-the chemists' round-bottomed flask of the 21st century?", Chem. Eur. J. 12, 8434-8442 (2006).

[2] G. M. Whitesides, "The origins and the future of microfluidics”, Nature 442, 368-373 (2006).

[3] J. M. Ottino and S. Wiggins, "Introduction: mixing in microfluidics", Phil. Trans. R. Soc. London. Ser. A 362, 923-935 (2004).

[4] D. Chiu, "Cellular manipulations in microvortices", Anal. Bioanal. Chem. 387, 17-20 (2007).

[5] D. Mark, S. Haeberle, G. Roth, F. von Stetten, and R. Zengerle, "Microfluidic lab-on-a-chip platforms: requirements, characteristics and applications", Chem. Soc. Rev. 39, 1153-1182 (2010).

[6] R. Feynman, "There is plenty room at the bottom", Engineering and Science 23 (5), 22-36 (1960).

[7] J.-C. Charpentier, "Four main objectives for the future of chemical and process engineering mainly concerned by the science and technologies of new materials production”, Chem. Eng. J. 107, 3 - 17 (2005).

[8] A. Thayer, "Harnessing microreactors", Chem. Eng. News 83 (22), 43-52 (2005).

[9] P. S. Dittrich and A. Manz, "Lab-on-a-chip: microfluidics in drug discovery", Nat. Rev. Drug Discov. 5, 210-218 (2006).

[10] A. Bennett, "Fine chemicals: Membrane technology in the fine chemicals industry", Filtration \& Separation 47, 16 - 19 (2010).

[11] P. L. Mills, D. J. Quiram, and J. F. Ryley, "Microreactor technology and process miniaturization for catalytic reactions: A perspective on recent developments and emerging technologies", Chem. Eng. Sci. 62, $6992-$ 7010 (2007).

[12] W. Ehrfeld, V. Hessel, and H. Löwe, Microreactors: New Technology for Modern Chemistry, chapter 1, 5-8 (Wiley-VCH Verlag GmbH \& Co. KGaA) (2000).

[13] T. M. Squires and S. R. Quake, "Microfluidics: Fluid physics at the nanoliter scale", Rev. Mod. Phys. 77, 977-1026 (2005).

[14] J. Edel and A. De Mello, Nanofluidics: Nanoscience and Nanotechnology, chapter 3, 47-63 (Royal Society of Chemistry) (2009).

[15] D. Fernandez Rivas, "Microfluidos: cuánto hay de nuevo?”, Rev. Cuban. Fis. 25, 142-149 (2008).

[16] D. Fernandez Rivas, "Microfluidos: Nuevas fronteras", Rev. Cuban. Fis. 28, pdf 60 (2011).

[17] D. Di Carlo, "Inertial microfluidics", Lab Chip 9, 3038-3046 (2009). 
[18] J. G. Santiago, "Electroosmotic flows in microchannels with finite inertial and pressure forces", Anal. Chem. 73, 2353-2365 (2001).

[19] H. A. Stone and S. Kim, "Microfluidics: Basic issues, applications, and challenges", AIChE J. 47, 1250-1254 (2001).

[20] K. S. Suslick and G. J. Price, “Applications of ultrasound to materials chemistry”, Annu. Rev. Mater. Sci. 29, 295-326 (1999).

[21] T. G. Leighton, "What is ultrasound?", Prog. Biophys. Mol. Biol. 93, 3 - 83 (2007).

[22] P. Cintas, S. Mantegna, E. C. Gaudino, and G. Cravotto, "A new pilot flow reactor for high-intensity ultrasound irradiation. application to the synthesis of biodiesel”, Ultrason. Sonochem. 17, 985 - 989 (2010).

[23] G. Cravotto, W. Bonrath, S. Tagliapietra, C. Speranza, E. C. Gaudino, and A. Barge, "Intensification of organic reactions with hybrid flow reactors", Chem. Eng. Process. 49, 930 - 935 (2010).

[24] K. Suslick, S. Doktycz, and E. Flint, "On the origin of sonoluminescence and sonochemistry", Ultrasonics 28, $280-290$ (1990).

[25] T. J. Mason and J. P. Lorimer, Applied Sonochemistry. The Uses of Power Ultrasound in Chemistry and Processing (Wiley-VCH Weinheim) (2002).

[26] C. Leonelli and T. J. Mason, "Microwave and ultrasonic processing: Now a realistic option for industry", Chem. Eng. Process. 49, 885 - 900 (2010).

[27] J. H. Bang and K. S. Suslick, "Applications of ultrasound to the synthesis of nanostructured materials", Adv. Mat. 22, 1039-1059 (2010).

[28] K. S. Suslick, D. A. Hammerton, and R. E. Cline, "Sonochemical hot spot”, J. Am. Chem. Soc. 108, 56415642 (1986).

[29] E. J. Hart, C. H. Fischer, and A. Henglein, "Pyrolysis of acetylene in sonolytic cavitation bubbles in aqueous solution", J. Phys. Chem. 94, 284-290 (1990).

[30] G. Cravotto and P. Cintas, "Power ultrasound in organic synthesis: moving cavitational chemistry from academia to innovative and large-scale applications", Chem. Soc. Rev. 35, 180-196 (2006).

[31] M. Margulis, "On the mechanism of multi-bubble sonoluminescence", Russ. J. Phys. Chem. 80, 1698-1702 (2006).

[32] K. S. Suslick, N. C. Eddingsaas, D. J. Flannigan, S. D. Hopkins, and H. Xu, "Extreme conditions during multibubble cavitation: Sonoluminescence as a spectroscopic probe", Ultrason. Sonochem. 18, $842-846$ (2011).

[33] H. Xu and K. S. Suslick, "Molecular emission and temperature measurements from single-bubble sonoluminescence”, Phys. Rev. Lett. 104, 244301 (2010).

[34] M. M. Caruso, D. A. Davis, Q. Shen, S. A. Odom, N. R. Sottos, S. R. White, and J. S. Moore, "Mechanicallyinduced chemical changes in polymeric materials", Chem. Rev. 109, 5755-5798 (2009).

[35] G. Cravotto and P. Cintas, "Molecular self-assembly and patterning induced by sound waves. the case of gelation”, Chem. Soc. Rev. 38, 2684-2697 (2009).

[36] G. Cravotto and P. Cintas, "Sonication-assisted fabrication and post-synthetic modifications of graphene-like materials”, Chem. Eur. J. 16, 5246-5259 (2010).

[37] G. Cravotto and P. Cintas, "Harnessing mechanochemical effects with ultrasound-induced reactions", Chem. Sci. 3, 295-307 (2012). 
[38] D. Fernandez Rivas, B. Verhaagen, J. Seddon, A. Zijlstra, L. Jiang, L. van der Sluis, M. Versluis, D. Lohse, and J. G. E. Gardeniers, "Localized removal of layers of metal, polymer or biomaterial by cavitating microbubbles", Biomicrofluidics 6, 034114 (2012).

[39] R. Karshafian, P. D. Bevan, R. Williams, S. Samac, and P. N. Burns, "Sonoporation by ultrasound-activated microbubble contrast agents: Effect of acoustic exposure parameters on cell membrane permeability and cell viability", Ultrasound Med. Biol. 35, 847 - 860 (2009).

[40] P. Riesz and T. Kondo, "Free radical formation induced by ultrasound and its biological implications", Free Radical Biol. Med. 13, 247-270 (1992).

[41] B. Ahmed-Omer, D. Barrow, and T. Wirth, "Effect of segmented fluid flow, sonication and phase transfer catalysis on biphasic reactions in capillary microreactors", Chem. Eng. J. 135, Supplement 1, S280 - S283 (2008).

[42] K. Suslick, Y. Didenko, M. Fang, T. Hyeon, K. Kolbeck, W. McNamara, M. Mdleleni, and M. Wong, "Acoustic cavitation and its chemical consequences", Philos. Trans. R. Soc. London, Ser. A 357, 335 (1999).

[43] S. Freitas, G. Hielscher, H. P. Merkle, and B. Gander, "Cone-like bubble formation in ultrasonic cavitation field", Ultrason. Sonochem. 13, 76 - 85 (2006).

[44] J. Friend and L. Y. Yeo, "Microscale acoustofluidics: Microfluidics driven via acoustics and ultrasonics", Rev. Mod. Phys. 83, 647-704 (2011).

[45] C. A. Speed, "Therapeutic ultrasound in soft tissue lesions”, Rheumatology 40, 1331-1336 (2001).

[46] B. Dollet, S. M. van der Meer, V. Garbin, N. de Jong, D. Lohse, and M. Versluis, "Nonspherical oscillations of ultrasound contrast agent microbubbles", Ultrasound Med. Biol. 34, 1465 - 1473 (2008).

[47] S. Song, Z. Shen, L. Chen, A. A. Brayman, and C. H. Miao, "Explorations of high-intensity therapeutic ultrasound and microbubble-mediated gene delivery in mouse liver", Gene Ther. 18, 1006-1014 (2011).

[48] T. Franke, A. R. Abate, D. A. Weitz, and A. Wixforth, "Surface acoustic wave (saw) directed droplet flow in microfluidics for pdms devices”, Lab Chip 9, 2625-2627 (2009).

[49] Z. Wang and J. Zhe, "Recent advances in particle and droplet manipulation for lab-on-a-chip devices based on surface acoustic waves", Lab Chip 11, 1280-1285 (2011).

[50] K. Kulkarni, J. Friend, L. Yeo, and P. Perlmutter, "Surface acoustic waves as an energy source for drop scale synthetic chemistry", Lab Chip 9, 754-755 (2009).

[51] J. Sedelmeier, S. V. Ley, I. R. Baxendale, and M. Baumann, "KMnO4-mediated oxidation as a continuous flow process", Org. Lett. 12, 3618-3621 (2010).

[52] R. L. Hartman, J. R. Naber, N. Zaborenko, S. L. Buchwald, and K. F. Jensen, "Overcoming the challenges of solid bridging and constriction during pd-catalyzed càín bond formation in microreactors", Org. Process Res. Dev. 14, 1347-1357 (2010).

[53] T. Noel, J. R. Naber, R. L. Hartman, J. P. McMullen, K. F. Jensen, and S. L. Buchwald, "Palladium-catalyzed amination reactions in flow: overcoming the challenges of clogging via acoustic irradiation", Chem. Sci. 2 , 287-290 (2011).

[54] R. L. Hartman, "Managing solids in microreactors for the upstream continuous processing of fine chemicals", Org. Process Res. Dev. 16, 870-887 (2012).

[55] T. Horie, M. Sumino, T. Tanaka, Y. Matsushita, T. Ichimura, and J.-i. Yoshida, "Photodimerization of maleic anhydride in a microreactor without clogging", Org. Process Res. Dev. 14, 405-410 (2010).

[56] S. Aljbour, H. Yamada, and T. Tagawa, "Ultrasound-assisted phase transfer catalysis in a capillary microreactor", Chem. Eng. Process. 48, 1167 - 1172 (2009). 
[57] S. Hübner, S. Kressirer, D. Kralisch, C. Bludszuweit-Philipp, K. Lukow, I. Jänich, A. Schilling, H. Hieronymus, C. Liebner, and K. Jähnisch, "Ultrasound and microstructures-a promising combination?", ChemSusChem 5, 279-288 (2012).

[58] J. Rooze, E. V. Rebrov, J. C. Schouten, and J. T. Keurentjes, "Effect of resonance frequency, power input, and saturation gas type on the oxidation efficiency of an ultrasound horn", Ultrason. Sonochem. 18, $209-215$ (2011).

[59] M. Plesset and A. Prosperetti, "Bubble dynamics and cavitation”, Annu. Rev. Fluid Mech. 9, 145-185 (1977).

[60] A. Prosperetti, "Bubble phenomena in sound fields: part one", Ultrasonics 22, 69 - 77 (1984).

[61] T. J. Mason, Practical Sonochemistry, A User's Guide to Applications in Chemistry and Chemical Engineering (Ellis Horwood Publishers, Chichester) (1991).

[62] T. Laurell, F. Petersson, and A. Nilsson, "Chip integrated strategies for acoustic separation and manipulation of cells and particles", Chem. Soc. Rev. 36, 492-506 (2007).

[63] F. Petersson, A. Nilsson, C. Holm, H. Jonsson, and T. Laurell, "Continuous separation of lipid particles from erythrocytes by means of laminar flow and acoustic standing wave forces”, Lab Chip 5, 20-22 (2005).

[64] J. Nilsson, M. Evander, B. Hammarström, and T. Laurell, "Review of cell and particle trapping in microfluidic systems", Anal. Chim. Acta 649, 141 - 157 (2009).

[65] G. G. Yaralioglu, I. O. Wygant, T. C. Marentis, and B. T. Khuri-Yakub, "Ultrasonic mixing in microfluidic channels using integrated transducers”, Anal. Chem. 76, 3694-3698 (2004), pMID: 15228343.

[66] F. Petersson, L. Åberg, A.-M. Swård-Nilsson, and T. Laurell, "Free flow acoustophoresis: Microfluidic-based mode of particle and cell separation", Anal. Chem. 79, 5117-5123 (2007), pMID: 17569501.

[67] R. Liu, J. Yang, M. Z. Pindera, M. Athavale, and P. Grodzinski, "Bubble-induced acoustic micromixing", Lab Chip 2, 151-157 (2002).

[68] P. Marmottant, J. Raven, H. Gardeniers, J. Bomer, and S. Hilgenfeldt, "Microfluidics with ultrasound-driven bubbles", J. Fluid Mech. 568, 109-118 (2006).

[69] D. Ahmed, X. Mao, J. Shi, B. K. Juluri, and T. J. Huang, “A millisecond micromixer via single-bubble-based acoustic streaming", Lab Chip 9, 2738-2741 (2009).

[70] P. Rogers and A. Neild, "Selective particle trapping using an oscillating microbubble", Lab Chip 11, 3710 3715 (2011).

[71] Y. Iida, T. Tuziuti, K. Yasui, A. Towata, and T. Kozuka, "Bubble motions confined in a microspace observed with stroboscopic technique”, Ultrason. Sonochem. 14, 621 - 626 (2007).

[72] J. Lee, T. Tuziuti, K. Yasui, S. Kentish, F. Grieser, M. Ashokkumar, and Y. Iida, "Influence of surface-active solutes on the coalescence, clustering, and fragmentation of acoustic bubbles confined in a microspace", J. Phys Chem. C 111, 19015-19023 (2007).

[73] Tandiono, S.-W. Ohl, D. S.-W. Ow, E. Klaseboer, V. V. T. Wong, A. Camattari, and C.-D. Ohl, "Creation of cavitation activity in a microfluidic device through acoustically driven capillary waves", Lab Chip 10, 1848-1855 (2010).

[74] S. Kuhn, T. Noël, L. Gu, P. L. Heider, and K. F. Jensen, "A teflon microreactor with integrated piezoelectric actuator to handle solid forming reactions", Lab Chip 11, 2488-2492 (2011).

[75] J.-L. Luche, Synthetic Organic Sonochemistry (Plenum Press, New York) (1998). 
[76] K. Yasuda, T. Torii, K. Yasui, Y. Iida, T. Tuziuti, M. Nakamura, and Y. Asakura, "Enhancement of sonochemical reaction of terephthalate ion by superposition of ultrasonic fields of various frequencies", Ultrason. Sonochem. 14, 699-704 (2007).

[77] M. Ashokkumar, D. Sunartio, S. Kentish, R. Mawson, L. Simons, K. Vilkhu, and C. Versteeg, "Modification of food ingredients by ultrasound to improve functionality: A preliminary study on a model system", Innov. Food Sci. Emerg, Technol. 9, 155-160 (2008).

[78] T. Tuziuti, K. Yasui, T. Kozuka, A. Towata, and Y. Iida, "Enhancement of sonochemical reaction rate by addition of micrometer-sized air bubbles", J. Phys. Chem. A 110, 10720-10724 (2006).

[79] D. Fernandez Rivas, A. Prosperetti, A. G. Zijlstra, D. Lohse, and H. J. G. E. Gardeniers, "Efficient sonochemistry through microbubbles generated with micromachined surfaces", Angew. Chem. Int. Ed. 49, 9699-9701 (2010).

[80] D. Flannigan and K. Suslick, "Plasma formation and temperature measurement during single-bubble cavitation”, Nature 434, 52-55 (2005).

[81] R. Pflieger, H. Brau, and S. Nikitenko, "Sonoluminescence from $\mathrm{OH}(\mathrm{C} 2 \Sigma+)$ and $\mathrm{OH}(\mathrm{A} 2 \Sigma+)$ Radicals in Water: Evidence for Plasma Formation during Multibubble Cavitation”, Chem. Eur. J. 16, 11801-11803 (2010).

[82] Y. T. Didenko and K. S. Suslick, "The energy efficiency of formation of photons, radicals and ions during single-bubble cavitation", Nature 418, 394-397 (2002).

[83] S. Hatanaka, H. Mitome, K. Yasui, and S. Hayashi, "Single-bubble sonochemiluminescence in aqueous luminol solutions", J. Am. Chem. Soc. 124, 10250-10251 (2002).

[84] M. P. Brenner, S. Hilgenfeldt, and D. Lohse, "Single bubble sonoluminescence”, Rev. Mod. Phys. 74, 425484 (2002).

[85] D. Lohse, "Sonoluminescence-cavitation hots up", Nature 434, 33-34 (2005).

[86] M. Ashokkumar, "The characterization of acoustic cavitation bubbles: An overview", Ultrason. Sonochem. 18, $864-872(2011)$.

[87] M. Ashokkumar, "The optimization of sonochemical reactions - facts and factors", in Proceedings of the Meeting of the Japan Society of Sonochemistry (JSS) and The International Workshop on Advanced Sonochemistry. November, Nagoya, Japan, S3 (2011).

[88] G. Andaluri, E. V. Rokhina, and R. P. Suri, "Evaluation of relative importance of ultrasound reactor parameters for the removal of estrogen hormones in water", Ultrason. Sonochem. 19, 953 - 958 (2012).

[89] N. Golash and P. R. Gogate, "Degradation of dichlorvos containing wastewaters using sonochemical reactors”, Ultrason. Sonochem. 19, 1051-1060 (2012).

[90] Y. Son, M. Lim, J. Khim, and M. Ashokkumar, "Acoustic emission spectra and sonochemical activity in a 36 khz sonoreactor”, Ultrason. Sonochem. 19, 16 - 21 (2012).

[91] Y. Iida, K. Yasui, T. Tuziuti, M. Sivakumar, and Y. Endo, "Ultrasonic cavitation in microspace", Chem. Commun. 2280-2281 (2004).

[92] A. Coleman, T. Kodama, M. Choi, T. Adams, and J. Saunders, "The cavitation threshold of human tissue exposed to 0.2-mhz pulsed ultrasound: Preliminary measurements based on a study of clinical lithotripsy", Ultrasound Med. Biol. 21, 405 - 417 (1995).

[93] Tandiono, S. Ohl, D. Ow, E. Klaseboer, V. Wong, R. Dumke, and C. Ohl, "Sonochemistry and sonoluminescence in microfluidics", Proc. Natl. Acad. Sci. USA 108, 5996-5998 (2011). 
[94] N. Bremond, M. Arora, C. D. Ohl, and D. Lohse, "Controlled multi-bubble surface cavitation", Phys. Rev. Lett. 96, 224501 (2006).

[95] D. Fernandez Rivas, M. Ashokkumar, T. Leong, K. Yasui, T. Tuziuti, S. Kentish, D. Lohse, and H. J. G. E. Gardeniers, "Sonoluminescence and sonochemiluminescence from a microreactor", Ultrason. Sonochem. 19, 1252-1259 (2012).

[96] D. Fernandez Rivas, L. Stricker, A. G. Zijlstra, H. J. G. E. Gardeniers, D. Lohse, and A. Prosperetti, "Ultrasound artificially nucleated microbubbles and their sonochemical radical production", Ultrason. Sonochem. in press, - (2012).

[97] R. Groote, B. M. Szyja, E. A. Pidko, E. J. M. Hensen, and R. P. Sijbesma, "Unfolding and mechanochemical scission of supramolecular polymers containing a metaläiligand coordination bond", Macromolecules 44, 9187-9195 (2011).

[98] D. Fernandez Rivas, R. Groote, and R. Jakobs, "Ring closing metathesis in a microsonoreactor", Unpublished results.

[99] A. Piermattei, S. Karthikeyan, and R. P. Sijbesma, "Activating catalysts with mechanical force", Nat. Chem. 1, 133-137 (2009).

[100] B. P. Mason, K. E. Price, J. L. Steinbacher, A. R. Bogdan, and D. T. McQuade, "Greener approaches to organic synthesis using microreactor technology", ChemInform 38(36) (2007).

[101] N. G. Anderson, "Using continuous processes to increase production”, Org. Process Res. Dev. 16, 852-869 (2012). 


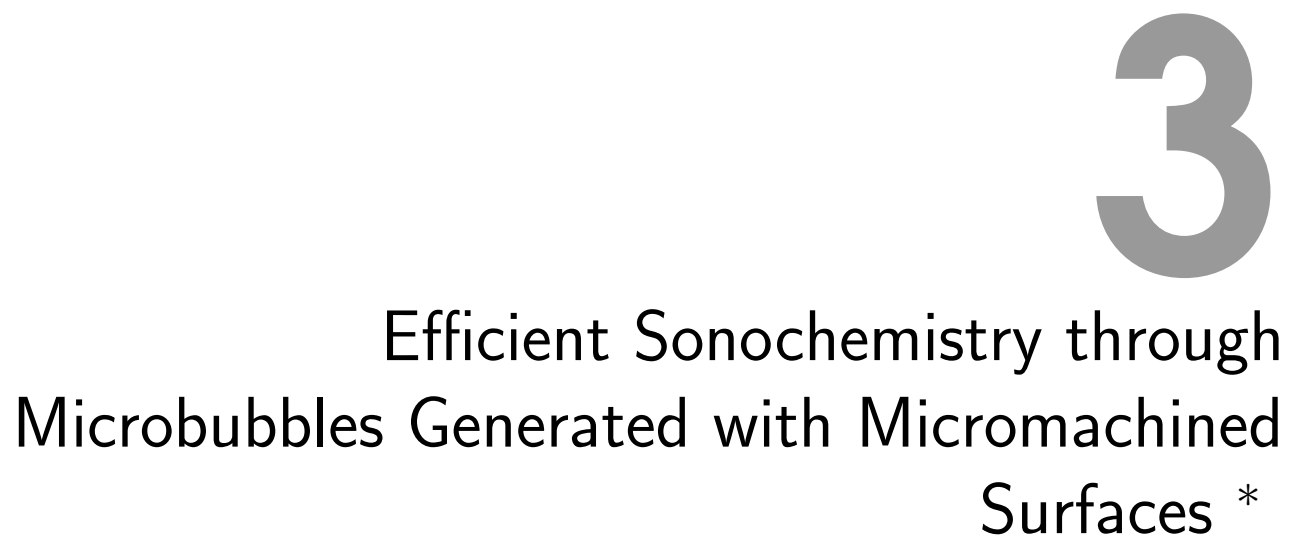

\begin{abstract}
Sonochemical reactors are used in water treatment, the synthesis of fine chemicals, pharmaceutics and others. The low efficiency of sonoreactors have prevented its massive usage at industrial scales. Controlling the appearance of bubbles in place and time is the most limiting factor. A novel type of sonochemical reactor was designed making use of micro-fabrication techniques to control the nucleation sites of micro-bubbles. The efficiency was increased first by locating the nucleation sites in the most active region of a micro-chamber; additionally the desired chemical effect was significantly higher at the same powers than when not controlled. Silicon substrates were micromachined with "artificial nucleation sites" or pits, and placed at the bottom of the micro-chamber. The pits entrap gas which, upon ultrasonic excitation, sheds off a stream of microbubbles. The gas content of the pits is not depleted but is replenished by diffusion and the emission of microbubbles can continue for hours.
\end{abstract}

*Published as: David Fernandez Rivas, Andrea Prosperetti, Aaldert G. Zijlstra, Detlef Lohse and Han J. G. E. Gardeniers, Efficient Sonochemistry through Microbubbles Generated with Micromachined Surfaces, Angewandte Chemie International Edition, 49:50, (2010), 9699-9701 


\subsection{Introduction}

The phenomenon of cavitation, that is, the growth and implosion of gas/vapor bubbles in a liquid, is a process which can locally generate extreme temperatures of several thousand Kelvin [1] and, for this reason, is exploited in sonochemistry to enhance chemical conversion. This feature opens the perspective of high-temperature, highpressure large scale systems and therefore holds the promise of constituting a "green chemistry" with a multitude of possible applications in water treatment [2], material synthesis, the food industry [3] and others. The "ideal" sonochemical reactor from a theoretical point of view is a single bubble trapped in an acoustically driven flask, such as in single bubble sonoluminescence [4]. There the bubble, in which argon accumulates [5], collapses periodically and in a reproducible way, as the collapse does not desintegrate the bubble. Reactants are sucked into the bubble at expansion and reaction products leave the bubble at collapse; typical temperatures achieved are $15000 \mathrm{~K}$ [6, 7]. Another advantage is that thanks to these ideal conditions the problem is accessible to a thorough theoretical treatment whose results are in good agreement with the experimental findings [4, 6]. The downside is that the absolute chemical yields are only tiny, as the ambient size of such bubbles is in the micrometer regime. For applications, typical sonochemical reactors, such as ultrasonic baths or vessels with ultrasound horns attached to their walls, are considerably larger than the active region in which cavitation occurs, which is defined by the ultrasound field that the transducer generates in the reactor. The difficulty of matching the acoustic cavitation activity with the reactor dimensions so that the complete volume of reactant can be stimulated adds to the low efficiency of most sonochemical processes. Attempts aimed at configuration optimization have mostly had limited success [8-11].

In this chapter a new approach is presented to address this problem based on the premise that a significant gain in efficiency may be obtained if the location of the nucleation of bubbles, which subsequently will cavitate due to ultrasound, can be accurately controlled. Achieving this objective will give us control over the spatial distribution of cavitation events, and therewith also over the actual volume of liquid that can be exposed to sonochemical effects.

The nucleation and formation of bubbles due to ultrasound is mostly heterogeneous, that is, it relies on pockets of gas trapped and stabilized inside randomly existing crevices in container walls or particles suspended in the bulk fluid rendering it extremely difficult to control and predict. The theory developed for bubble nucleation from crevices [12, 13] leads the way to control nucleation. As shown in recent work [13-16], stable and monodisperse cavitation nuclei can be formed by trapping gas in pits micromachined in a silicon surface. We use such pits which serve as artificial crevices for the inception of cavitation to achieve higher sonochemical yields at ultrasound powers which would otherwise not produce a significant chemical effect. 


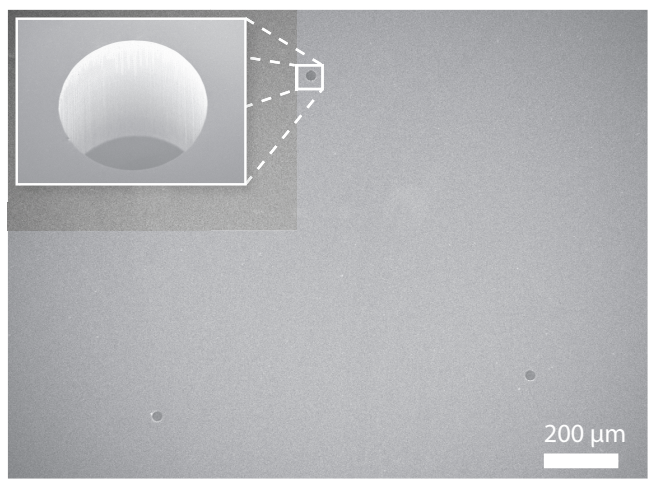

Figure 3.1: Pits etched in a silicon substrate. Top view of the 3-pit set with $30 \mu \mathrm{m}$ diameter micropits.

\subsection{Experimental methods and materials}

\subsubsection{Silicon micromachining:}

Three different configurations of pits were used. The pits had the same diameter (30 $\mu \mathrm{m}$ ) and were arranged in sets of one, two (in a line) or three (in a triangle, see Figure 3.1) at a distance of $1000 \mu \mathrm{m}$ from each other.

The substrates were micromachined under clean room conditions on double-side polished silicon wafers and spin coated with the photosensitive substance Olin 12, on which the designed pattern was transferred with a mask aligner EV620 (photolitography). After development the pit pattern was open and with a plasma dry-etching machine Adixen AMS 100 SE (Alcatel) process BHARS, the holes were etched into the silicon substrate at the desired depth. The machined diced silicon square pieces of $1 \mathrm{~cm}$-side were mounted to the bottom of a small glass container which contained a liquid volume of $300 \mu \ell$, to the bottom of which a piezo element was attached.

\subsubsection{The acoustic field}

The reaction chamber was a glass container of $2.5 \mathrm{~cm}$ outer diameter, $1.5 \mathrm{~cm}$ inner diameter and depth of $3 \mathrm{~mm}$, and bottom thickness of $2 \mathrm{~mm}$. the bottom thickness matched the quarter-wavelength vibration imparted by a piezo Ferroperm PZ27 $6 \mathrm{~mm}$ thick with diameter of $2.5 \mathrm{~cm}$, glued to the bottom of the reaction chamber. The top of the glass container was capped with a rubber ring and a glass slide to avoid evaporation of the sample during the experiments (see Figure 3.2). A sinusoidal standing acoustic field of $200 \mathrm{kHz}$ was generated by a Hewlett Packard model 33120A arbitrary waveform generator, amplified with a SONY TA-FB74OR QS amplifier. The voltage, current and phase difference provided to the piezoelectric element were mea- 


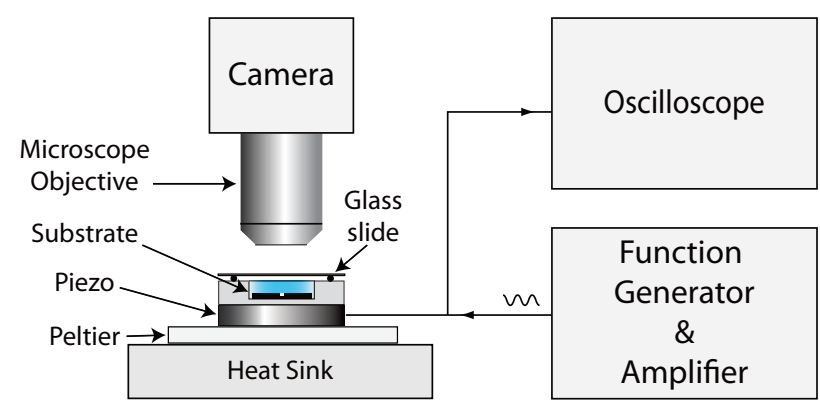

Figure 3.2: Experimental setup

sured with a Tektronix DPO 4034 oscilloscope and Tektronix current probe CTA-2.

Three different power settings were used: low $(74 \mathrm{~mW})$, medium $(182 \mathrm{~mW})$ and high $(629 \mathrm{~mW})$. During the experiments, the bottom surface of the piezoelectric element was in contact with a Peltier element (Marlow Industrial) to keep the temperature at a constant value of approximately $25^{\circ} \mathrm{C}$.

\subsubsection{Luminol visualization and determination of the radical formation rate}

The homolytic cleavage of $\mathrm{H}_{2} \mathrm{O}$ by sonolysis was investigated using Luminol and terephthalic acid. Luminol is a well-established indicator for the visualization of active sonochemical regions, because it reacts with $\mathrm{OH}$ radicals, whereupon reaction gives luminescence with an intensity proportional to the amount of radicals produced [17]. A solution of $1 \times 10^{-3} \mathrm{~mol} / \ell$ Luminol and $1 \times 10^{-4} \mathrm{~mol} / \ell$ hydrogen peroxide was prepared with adjusted $\mathrm{pH}=12$ by adding $\mathrm{Na}_{2} \mathrm{CO}_{3}$, as described in [18].

Conversion of terephthalic acid to 2-hydroxyterephthalic acid (HTA) was taken as a quantitative measure for the concentration of $\mathrm{OH}$ radicals formed by the ultrasound induced bubble activity. A calibration graph for the fluorescence intensity as a function of HTA concentration was obtained following steps similar to those described in [19]. Fluorescence was measured using a spectrofluorometer (TECAN Sapphire) with an excitation wavelength of $310 \mathrm{~nm}$ and an emission wavelength of $429 \mathrm{~nm}$. A graph of fluorescence intensity against HTA concentration was plotted and gave a straight line of positive slope for concentrations. The Terephthalic acid solution to be used as dosimeter was prepared by mixing $0.332 \mathrm{~g}$ of Terephthalic acid (Sigma-Aldrich, $\left.2.0 \times 10^{-3} \mathrm{~mol} / \ell\right), 0.200 \mathrm{~g}$ of $\mathrm{NaOH}\left(5.0 \times 10^{-3} \mathrm{~mol} / \ell\right)$, and phosphate buffer $(\mathrm{pH}$ 7.4), prepared from $0.589 \mathrm{~g}$ of $\mathrm{KH}_{2} \mathrm{PO}_{4}\left(4.4 \times 10^{-3} \mathrm{~mol} / \ell\right)$ and $0.981 \mathrm{~g}$ of $\mathrm{Na}_{2} \mathrm{HPO}_{4}$ $\left(7.0 \times 10^{-3} \mathrm{~mol} / \ell\right.$, all from Riedel - de Haën). The resulting solution was then made up to $1 \ell$ with water [20]. For each experiment run, the same amount of terephthalic acid solution $300 \mu \ell$ measured with Eppendorf micropipettes was used. 
At the end of the experimental run, the solution was pipetted out of the reaction chamber and stored in the dark in sterilized vials (manufactured by Brand) for ultrapure chemical analysis for further spectroscopic analysis. Later, $200 \mu \ell$ taken from the samples were pipetted into the wells of an assay plate (Corning Inc.) to be analyzed with the spectrofluorometer described above. The conditions for sample analysis were: gain 40, height from the well, $9000 \mu \mathrm{m}$, integration time $100 \mu \mathrm{s}, 10$ reads per well. Excitation was at $310 \mathrm{~nm}$ and the emission scan was from 350 to 600 $\mathrm{nm}$. Each experiment was repeated at least 6 times showing acceptable consistency.

Calculation of the radical formation rate was done according to:

$$
\frac{d N_{\text {rad }}}{d t}=\frac{\left(c_{\text {end }}-c_{\text {initial }}\right) N_{A} V}{\Delta t}
$$

Here $c_{\text {end }}$ and $c_{\text {initial }}$ are the final and initial concentration in moles per volume, $N_{A}=6.02 \times 10^{23} \mathrm{~mol}^{-1}$ is Avogadro's number and $V$ is the liquid volume exposed to the ultrasound $(300 \mu \ell)$. With $c_{\text {initial }}=0$ this relation provides an estimate of the rate of radical formation for all pits and power combinations.

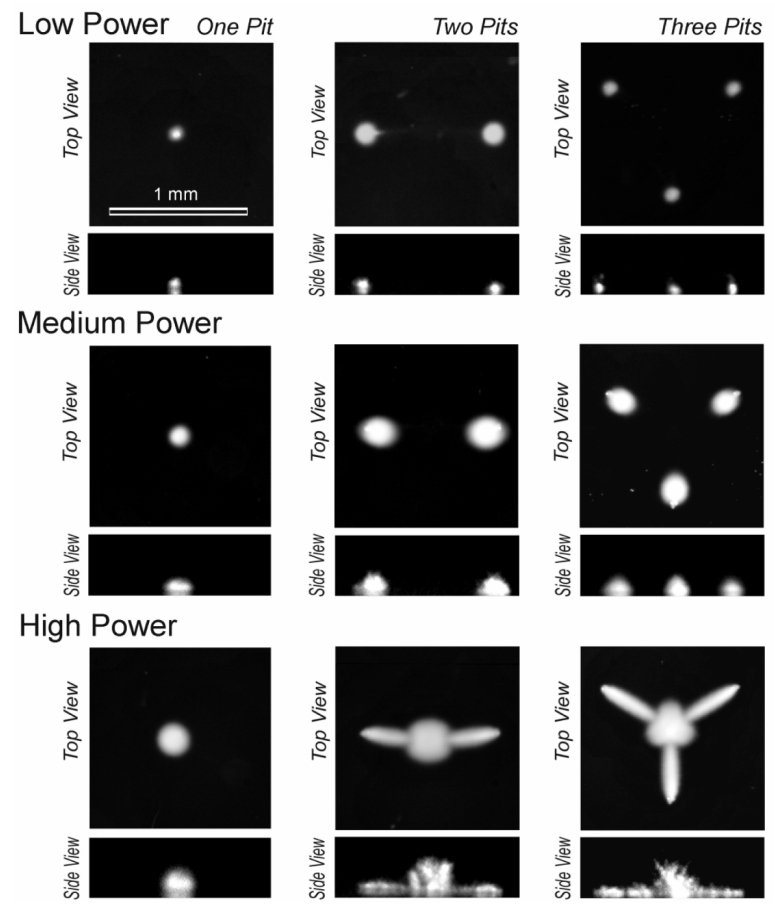

Figure 3.3: Images showing both the top and side view of the bubble structures generated from the micropits for different configurations (1,2 and 3 pits) and for increasing power level. Low power corresponds to $74 \mathrm{~mW}$, medium power to $182 \mathrm{~mW}$, and high power to $629 \mathrm{~mW}$. 


\subsection{Results}

The micropit bubble ejects streams of microbubbles, which resemble previously reported streamers [21, 22]. The observed trajectories in Figure 3.3 are the result of the complex interplay of primary and secondary Bjerknes forces and microstreaming. For a single pit, at low and medium ultrasound powers, the microbubbles are ejected and oscillate several cycles until they dissolve or are recaptured by the micropit bubble. For low power the situation is similar for the configurations with two and three pits. With increasing power a drastic transition in the bubble flow pattern is observed. Beyond the transition the microbubbles move away from the symmetry axis of their respective pits and towards one another (see intermediate row in Figure 3.3). The microbubbles then form a dense bubble cloud traveling towards the midpoint between the two-pit or to the midpoint of the three-pit arrangement. For the three-pit configuration the microstreamers point to the center of the triangular array and form a triangular cloud of bubbles, as seen in top view.

These experiments were repeated with the water solution of luminol. In Figure 3.4 the resulting images can be seen. Imaging of the low intensity of the emitted luminescence required the use of long camera exposure times (typically 30 seconds). A detailed comparison of the features in Figure 3.4 with those in Figure 3.3 shows a perfect match. Clearly, the regions of cavitation activity correspond to the regions where light is emitted due to sonochemiluminescence [17].

For a quantitative measure of radical formation, the terephthalic acid dosimetry method was used. The fluorescence intensity emission of HTA at $429 \mathrm{~nm}$ (measured ex-situ) allows us to calculate the amount of radicals generated by ultrasound induced microbubble cavitation. This measurement was performed for the three pit configurations, each at low, medium and high power and for 15 and 30 minutes process durations. In addition, experiments with the same conditions were done with a silicon chip without micropits. These measurements showed no significant evidence of radical formation. The radical generation rates (equation 6.1) resulting from off-line fluorescence measurements are shown in Figure 3.5 .

The data show that the presence of pits gives a significant enhancement of the radical formation rate. The rate is stable over time and is an increasing power of the ultrasound field as expected. Radical formation increases with the number of pits, but the slope of the lines decreases as more pits are added, presumably due to the onset of interactions with the bubble clouds generated by the other pits. At high power, the bubble pattern changes dramatically (Figures 3.3 and 3.4) and is expected to lead to a different radical generation distribution over the reactor volume. The quantitative interpretation of these data is complex. Generally speaking, smaller bubbles are stiffer due to a surface tension contribution and may not grow as large during expansion, with a consequent weaker compression and lower maximum temperatures. Large bubbles, on the other hand, may not collapse spherically, especially when close to 


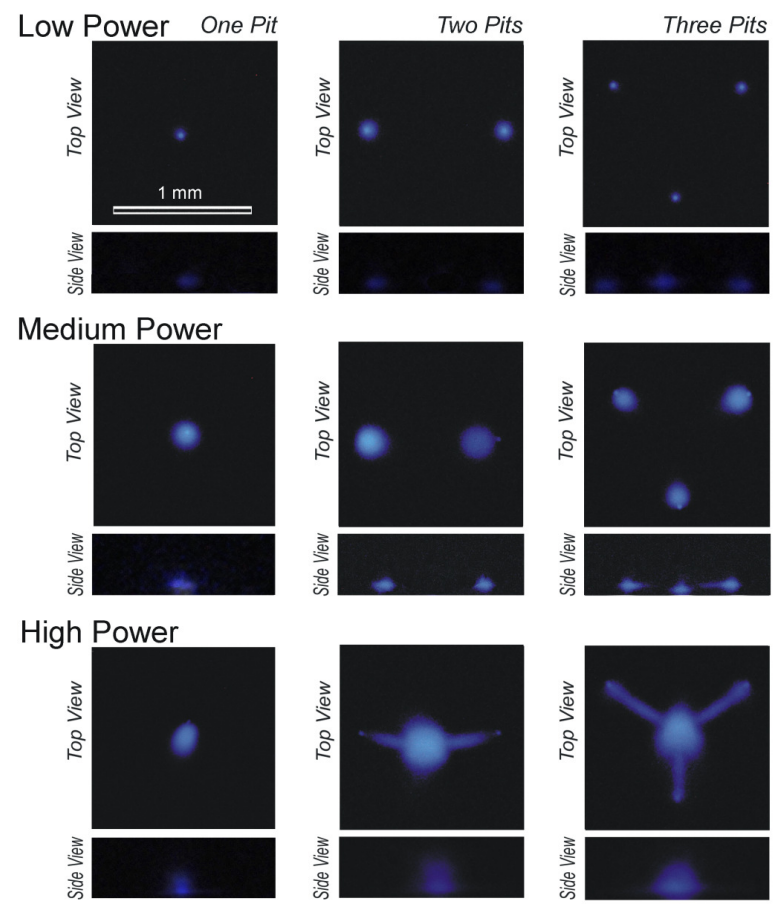

Figure 3.4: Luminol luminescence in dark room conditions. Images showing both the top and side view of the bubble structures generated from the micropits for different configurations (1,2 and 3 pits) and for increasing power level. Compare with Figure 3.3

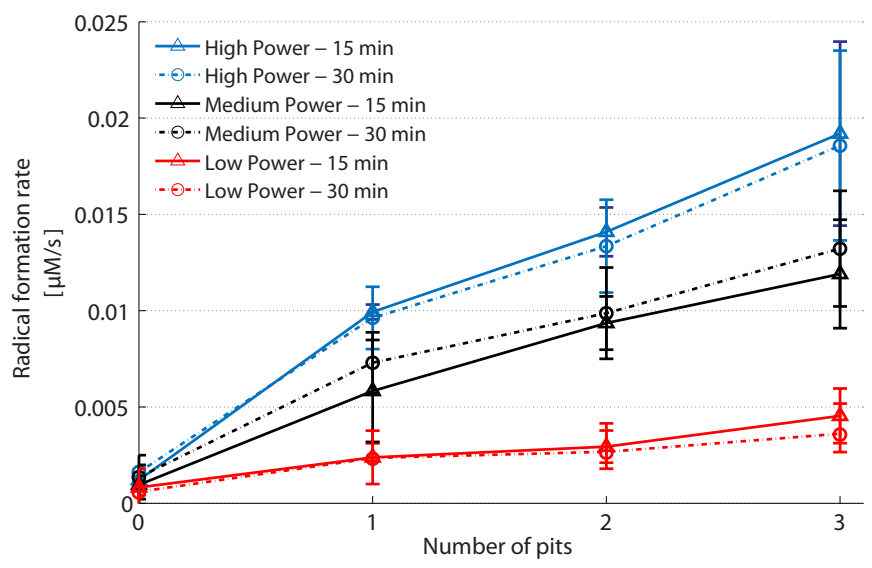

Figure 3.5: Radical formation rate vs. pits. Low power $=74 \mathrm{~mW}$, medium power $=182 \mathrm{~mW}$ and high power $=629 \mathrm{~mW}$. The bars show the range of the experimental data 
a solid surface, which would also limit their maximum compression. It is not clear, however, to what extent these general trends are relevant in the experimental situation of present concern.

The most important parameter for the evaluation of our results and comparison with the work of others is the energy efficiency defined as:

$$
X_{U S}=\frac{\Delta H}{P_{U S}} \frac{d N_{r a d}}{d t}
$$

where $\frac{d N_{\text {rad }}}{d t}$ is the radical formation rate in moles per second, $\Delta H$ is the energy required for the formation of $\mathrm{OH} \cdot$ radicals, which is equal to the enthalpy of formation associated with the following chemical reaction:

$$
\mathrm{H}_{2} \mathrm{O} \stackrel{\Delta H=-5.1 \mathrm{eV}}{\rightarrow} \mathrm{OH}+\mathrm{H}^{\cdot}
$$

This enthalpy has a value of $5.1 \mathrm{eV}$ per molecule [23]. $P_{U S}$ is the electric power absorbed by the transducer which can be obtained from the measured voltage, current and their phase difference. The efficiency as defined in Equation 6.2 is shown in Table 3.1 for each of the configurations studied.

Table 3.1: Efficiency $\times 10^{6}$ as defined in equation 6.2

\begin{tabular}{cllll}
\hline $\begin{array}{c}\text { High power } \\
629 \mathrm{~mW}\end{array}$ & 3 pits & 2 pits & 1 pit & 0 pit \\
\hline $15 \mathrm{~min}$ & 4.5 & 3.3 & 2.3 & 0.3 \\
$30 \mathrm{~min}$ & 4.4 & 3.1 & 2.3 & 0.4 \\
\hline $\begin{array}{c}\text { Medium power } \\
182 \mathrm{~mW}\end{array}$ & 3 pits & 2 pits & 1 pit & 0 pit \\
\hline $15 \mathrm{~min}$ & 9.7 & 7.6 & 4.7 & 0.8 \\
$30 \mathrm{~min}$ & 11 & 8.0 & 5.9 & 0.1 \\
\hline Low power & & & & \\
$74 \mathrm{~mW}$ & 3 pits & 2 pits & 1 pit & 0 pit \\
\hline $15 \mathrm{~min}$ & 9.1 & 5.9 & 4.8 & 1.6 \\
$30 \mathrm{~min}$ & 7.1 & 5.2 & 4.6 & 1.1 \\
\hline
\end{tabular}

\subsection{Discussion and Conclusions}

From the values in Table 3.1 we can draw the following conclusions: (i) Comparing the efficiency values of the chips with one or several pits with those of the chip with 
no pits, there is an efficiency increase by an order of magnitude, which demonstrates that the pits on the reactor wall give a considerable enhancement. (ii) The efficiency obtained even with such a low number of pits is close to the highest efficiencies reported in the literature with conventional sonochemical reactors [20, 24,-30]. A detailed comparison is hardly possible due to the use of different frequencies, chemical dosimeters and other physical parameters.

From the values of Table 3.1 it can be seen that the most efficient setting is medium power in all pit configurations. Several factors may explain this observation. At high power, the microbubble collapse is more catastrophic which results in a smaller compression and heating of the gas. In the case of two- and three-pit configuration, the microbubbles stay longer in the most active zone close to the substrate while traveling parallel to the surface; but the collapse of microbubbles close to the surface is highly non spherical, again reducing the maximum compression and heating of the gas. The microbubbles in the dense cloud at the midpoint may also have a weaker collapse due to the shielding by the outer microbubbles.

It has to be stressed that our results were obtained with only a very small active region, with state-of-the-art microfabrication procedures it is straightforward to increase the number of pits (see Figure 3.6. Based on our results in Table 3.1 and Figure 3.5, increasing the number of pits by a factor of ten should give an increase in energy efficiency by a factor of 100 or more. This estimate is based on a linear extrapolation of the one-, two- and three-pit configuration at medium power settings.
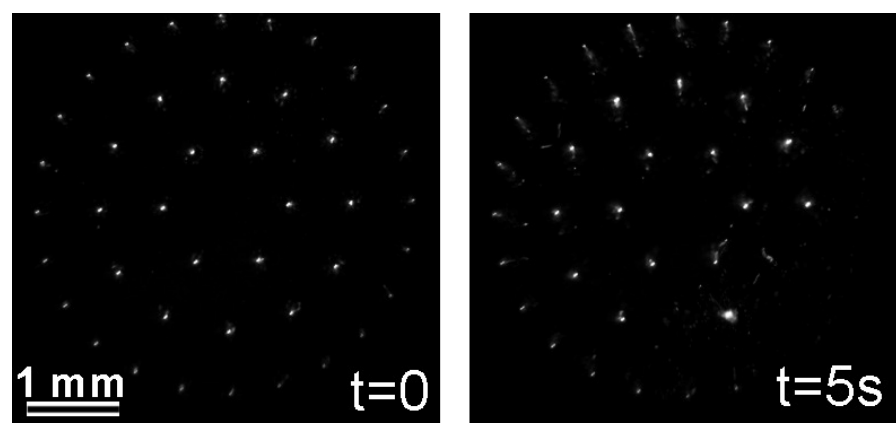

Figure 3.6: Microscope image under normal light conditions showing an arrangement with a higher number of pits (42). Here similar ejections of microbubbles from the micropit bubbles occur as described in this chapter.

Our results indicate that the introduction of micromachined pits on the surface of a reactor wall attached to an ultrasound transducer can boost sonochemical efficiency. The underlying mechanism is the production of microbubble cavitation clouds from the stable gas pockets that form in these pits. 


\section{References}

[1] K. S. Suslick and D. J. Flannigan, "Inside a collapsing bubble: Sonoluminescence and the conditions during cavitation”, Annu. Rev. Phys. Chem. 59, 659-683 (2008).

[2] P. R. Gogate, "Application of cavitational reactors for water disinfection: Current status and path forward", J. Environ. Manage. 85, 801-815 (2007).

[3] M. Ashokkumar, D. Sunartio, S. Kentish, R. Mawson, L. Simons, K. Vilkhu, and C. Versteeg, "Modification of food ingredients by ultrasound to improve functionality: A preliminary study on a model system", Innov. Food Sci. Emerg, Technol. 9, 155-160 (2008).

[4] M. P. Brenner, S. Hilgenfeldt, and D. Lohse, "Single bubble sonoluminescence”, Rev. Mod. Phys. 74, 425-484 (2002).

[5] D. Lohse, M. P. Brenner, T. F. Dupont, S. Hilgenfeldt, and B. Johnston, "Sonoluminescing air bubbles rectify argon”, Phys. Rev. Lett. 78, 1359-1362 (1997).

[6] R. Toegel and D. Lohse, "Phase diagrams for sonoluminescing bubbles: A comparison between experiment and theory", J. Chem. Phys. 118, 1863 (2003).

[7] D. J. Flannigan and K. S. Suslick, "Plasma formation and temperature measurement during single-bubble cavitation", Nature 434, 52-55 (2005).

[8] K. Yasuda, T. Torii, K. Yasui, Y. Iida, T. Tuziuti, M. Nakamura, and Y. Asakura, "Enhancement of sonochemical reaction of terephthalate ion by superposition of ultrasonic fields of various frequencies", Ultrason. Sonochem. 14, 699-704 (2007).

[9] Z. Xu, C. Y. Ma, J. Y. Xu, and X. J. Liu, "Dynamical properties of iodine release in potassium iodide solution under combination of ultrasound and light irradiations”, Ultrason. Sonochem. 16, 475-480 (2009).

[10] S. Koda, K. Tanaka, H. Sakamoto, T. Matsuoka, and H. Nomura, "Sonochemical efficiency during singlebubble cavitation in water”, J. Phys. Chem. A 108, 11609-11612 (2004).

[11] T. Tuziuti, K. Yasui, T. Kozuka, A. Towata, and Y. Iida, "Enhancement of sonochemical reaction rate by addition of micrometer-sized air bubbles”, J. Phys. Chem. A 110, 10720-10724 (2006).

[12] A. A. Atchley and A. Prosperetti, "The crevice model of bubble nucleation”, J. Acoust. Soc. Am. 86, 10651084 (1989)

[13] B. M. Borkent, S. Gekle, A. Prosperetti, and D. Lohse, "Nucleation threshold and deactivation mechanisms of nanoscopic cavitation nuclei”, Phys. Fluids 21 (2009).

[14] N. Bremond, M. Arora, C. D. Ohl, and D. Lohse, "Controlled multi-bubble surface cavitation", Phys. Rev. Lett. 96, 224501 (2006).

[15] N. Bremond, M. Arora, S. M. Dammer, and D. Lohse, "Interaction of cavitation bubbles on a wall", Phys. Fluids 18 (2006).

[16] P. Marmottant, J. P. Raven, H. Gardeniers, J. G. Bomer, and S. Hilgenfeldt, "Microfluidics with ultrasounddriven bubbles", J. Fluid Mech. 568, 109-118 (2006).

[17] A. Henglein, R. Ulrich, and J. Lilie, "Luminescence and chemical action by pulsed ultrasound", J. Am. Chem. Soc. 111, 1974-1979 (1989).

[18] B. Felver, D. C. King, S. C. Lea, G. J. Price, and A. D. Walmsey, "Cavitation occurrence around ultrasonic dental scalers", Ultrason. Sonochem. 16, 692-697 (2009).

[19] T. J. Mason, J. P. Lorimer, D. M. Bates, and Y. Zhao, "Dosimetry in sonochemistry - the use of aqueous terephthalate ion as a fluorescence monitor", Ultrason. Sonochem. 1, S91-S95 (1994). 
[20] Y. Iida, K. Yasui, T. Tuziuti, and M. Sivakumar, "Sonochemistry and its dosimetry", Microchem. J. 80, 159164 (2005).

[21] T. G. Leighton, "Bubble population phenomena in acoustic cavitation", Ultrason. Sonochem. 2, S123-S136 (1995).

[22] E. A. Neppiras, “Acoustic cavitation”, Phys. Rep. 61, 159-251 (1980).

[23] R. Toegel, S. Hilgenfeldt, and D. Lohse, "Suppressing dissociation in sonoluminescing bubbles: The effect of excluded volume”, Phys. Rev. Lett. 88 (2002).

[24] Y. T. Didenko and K. S. Suslick, "The energy efficiency of formation of photons, radicals and ions during single-bubble cavitation”, Nature 418, 394-397 (2002).

[25] M. W. A. Kuijpers, M. F. Kemmere, and J. T. F. Keurentjes, "Calorimetric study of the energy efficiency for ultrasound-induced radical formation”, Ultrasonics 40, 675-678 (2002).

[26] A. Rochebrochard, J. Blais, and E. Naffrechoux, "Comparison of characterization methods in high frequency sonochemical reactors of differing configurations.”, Ultrason. Sonochem. 17, 547-554 (2010).

[27] V. S. Sutkar and P. R. Gogate, "Design aspects of sonochemical reactors: Techniques for understanding cavitational activity distribution and effect of operating parameters", Chem. Eng. J. 155, 26-36 (2009).

[28] L. Hallez, F. Touyeras, J. Y. Hihn, J. Klima, J. L. Guey, M. Spajer, and Y. Bailly, "Characterization of hifu transducers designed for sonochemistry application: Cavitation distribution and quantification", Ultrasonics 50, 310-317 (2010).

[29] G. Mark, A. Tauber, L. A. Rudiger, H. P. Schuchmann, D. Schulz, A. Mues, and C. von Sonntag, "OH-radical formation by ultrasound in aqueous solution - Part II: Terephthalate and Fricke dosimetry and the influence of various conditions on the sonolytic yield", Ultrason. Sonochem. 5, 41-52 (1998).

[30] A. Mandroyan, R. Viennet, Y. Bailly, M. L. Doche, and J. Y. Hihn, "Modification of the ultrasound induced activity by the presence of an electrode in a sonoreactor working at two low frequencies (20 and $40 \mathrm{kHz}$ ). Part I: Active zone visualization by laser tomography", Ultrason. Sonochem. 16, 88-96 (2009). 


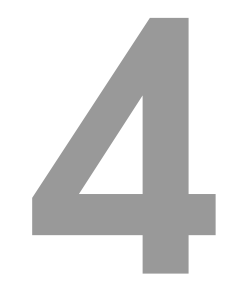

\title{
Sonoluminescence and Sonochemiluminescence from a Microreactor ${ }^{*}$
}

\begin{abstract}
Under suitable conditions, the collapse of bubbles nucleated from micromachined pits can result in light emission (sonoluminescence, SL). Hydroxyl radicals $(\mathrm{OH} \cdot$ ) generated during bubble collapse can react with luminol to produce light (sonochemiluminescence, SCL). SL and SCL intensities were recorded for several regimes related to the pressure amplitude (low and high acoustic power levels) at a given ultrasonic frequency ( $200 \mathrm{kHz}$ ) for pure water, and aqueous luminol and propanol solutions. Various arrangements of pits were studied, with the number of pits ranging from no pits (comparable to a classic ultrasound reactor), to three-pits. Where there was more than one pit present, in the high pressure regime the ejected microbubbles combined into linear (two-pits) or triangular (three-pits) bubble clouds (streamers). In all situations where a pit was present on the substrate, the SL was intensified and increased with the number of pits at both low and high power levels. For imaging SL emitting regions, Argon (Ar) saturated water was used under similar conditions. SL emission from aqueous propanol solution $(50 \mathrm{mM})$ provided evidence of transient bubble cavitation. Solutions containing $0.1 \mathrm{mM}$ luminol were also used to demonstrate the radical production by attaining the SCL emission regions.
\end{abstract}

*Published as: David Fernández Rivas, Muthupandian Ashokkumar, Thomas Leong, Kyuichi Yasui, Toru Tuziuti, Sandra Kentish, Detlef Lohse, Han J.G.E. Gardeniers, Sonoluminescence and sonochemiluminescence from a microreactor, Ultrasonics Sonochemistry, 6, 19, (2012), 1252-1259. 


\subsection{Introduction}

A well known effect of ultrasonic irradiation in a liquid is acoustic cavitation, which is the nucleation and consequent collapse of bubbles [1]. Sometimes, bubbles can cavitate in phase with the applied sound frequency. These bubbles behave as "individual micro-reactors", as they are often accompanied by a violent collapse that leads to high pressures and temperatures within and in the local vicinity of the bubbles [24]. Among the events generated during such collapses, some can be identified as plasma formation, lysis of molecules yielding radicals (water molecule sonolysis is a well known example), strong pressure shockwaves, liquid jetting and surface erosion. Although the driving condition is constant in most cases, the cavitation bubbles are never exactly the same due to the complexity of the phenomena involved [5], rendering it difficult to obtain a deterministic relation between driving conditions and actual bubble population. To complement direct determination of bubble size distributions which require fast imaging and intensive image processing, a good alternative is the use of indirect methods like measuring the light emitted by these bubbles.

Most researchers agree that the studies of Marinesco and Trillat [6] and Frenzel and Shultes [7] were the first in which light emission could be detected as a result of ultrasound irradiation in a liquid sample. After several studies to determine the actual mechanism behind this remarkable effect, sonoluminescence (SL) has been described as the light emitted by cavitation bubbles driven by an ultrasonic driving pressure field. Sonochemiluminescence (SCL) is defined in this study as the light emission when luminol reacts with $\mathrm{OH}^{*}$ radicals. There is a large difference between the SL emission from a single bubble (SBSL) and SL from a cluster of bubbles (MBSL, i.e. multibubble SL). For SBSL the collapse is nearly spherically symmetric and highly reproducible [4]; and for MBSL the more frequent non repeatable asymmetric collapse produces liquid jets penetrating the hot bubble contents [8-11]. These differences are evident in the emission profile and spectra produced from both types of SL. The spectra collected from MBSL contain many peaks and features, whereas the spectra emanating from SBSL are normally featureless [12, 13]. The effect of power and frequency on bubble-size distributions of MBSL in acoustic cavitation has been studied previously using pulsed ultrasound [14]. The main conclusion was that the mean bubble size increased with increasing acoustic power and the same time decreased with increasing ultrasound frequency. Additionally, the mean bubble size distribution of bubbles emitting SL was larger and narrower than SCL producing bubbles (centred at smaller bubble sizes and broader) meaning that the two processes result from different bubble sizes [15] and the physical locations in which these bubbles exist can differ [16]. MBSL has also a defined phase window of the driving pressure oscillation, meaning that in a phase window of about $30^{\circ}$ from the full $360^{\circ}$ SL can be detected [5]. Other researchers have reported singular dependences of SL and capillary pressure in small gaps with an implicit advantage in not using a 
light-proof box to quantify multibubble intertial cavitation thresholds [17].

Sonochemistry performed in microfluidic devices has received some attention in the last decade [18-21]. In this work an extended study on an ultrasonic microreactor described before [18] is presented. The working principle of that sonoreactor is based on the ability of small predefined crevices (pits etched in silicon substrate surface [22-25]) to stabilize small gas nuclei. When the ultrasound is turned on, a characteristic microbubble cloud appears, that would not be present in the absence of pits. In this way a continuous locally controlled generation of cavitating microbubbles is achieved. The chemical activity of these microbubbles was previously verified by luminol SCL imaging and $\mathrm{OH}$ radical dosimetry by using terephthalic acid [18].

The aim of the present work was to study the changes of SL and SCL intensities emanating from different solutions as the population of microbubbles nucleated from the pits on the silicon substrate varies. This is influenced by altering the power input to the system and the number of pits. The areas of potential application for these findings are many; to name a few we consider the ultrapurification of water for fine chemicals or pharmaceutical uses, mechanochemistry and the selective cleaning of circuit boards in which localized cavitation can avoid the damage of certain components with conventional sonication systems [26, 27]. Our results can be of importance to existing non destructive testing and inspection of surfaces with localized fluorescent dye penetration which are improved by the action of localized cavitation[28].

Additionally for biological applications where a localized source of radicals, light and streaming forces are required, this system might be beneficial [29, 30].

\subsection{Material and Methods}

This work focused on the measurement of the SL and SCL intensities emitted from three different systems. The first was air-saturated Milli-Q water, the second was air saturated propanol solution $(50 \mathrm{mM})$ in water and the third was air saturated aqueous luminol $(0.1 \mathrm{mM}$ luminol in $0.1 \mathrm{M} \mathrm{NaOH})$ solution. SL intensities were obtained for water and propanol, whereas SCL intensities were measured for luminol. Additionally SL images were recorded in argon saturated water.

In most cavitating systems, there exist populations of SL active and SCL active bubbles [31]. These populations strongly overlap: SL active bubbles can be SCL active, and vice versa. SL active bubbles correspond to bubbles that satisfy suitable conditions (pressure and temperature) inside the bubble that allow for ionization and the subsequent light emission [32]. SCL active bubbles produce radicals $\left(\mathrm{OH} \cdot{ }^{-}\right.$radicals in this case):

$$
\mathrm{H}_{2} \mathrm{O} \stackrel{\Delta H=-5.1 e V}{\rightarrow} \mathrm{OH}+\mathrm{H}^{\cdot}
$$

Recording the light emission from luminol molecules reacting with $\mathrm{OH}^{\cdot}$ is a 
widely used method to quantify the chemical activity and map active zones in a sonoreactor. Evidently knowing the exact bubble size distribution and their spatial localization is very difficult ([33-35] ), and most studies are based on bubble dissolution when US is turned off [14, 36, 37]. By virtue of dissolved propanol in water, information about the presence of transient cavitating bubbles can be obtained [38, 39]. It has been shown that alcohols do not quench SL arising from transient cavitation (i.e., MBSL), as the alcohol molecules do not have enough time to accumulate on the interface of the transiently cavitating bubbles [39]. That is in vast contrast to stable SBSL, where alcohols strongly quench the light emission [40].

\subsubsection{Set-up for US experiments}

A scheme of the experimental setup used is shown in Figure 6.2. The reaction chamber was a glass container of $25 \mathrm{~mm}$ outer diameter, $15 \mathrm{~mm}$ inner diameter and depth of $2 \mathrm{~mm}$, and bottom thickness of $2 \mathrm{~mm}$. The bottom thickness matched the quarterwavelength vibration imparted by a piezo Ferroperm PZ27 $6 \mathrm{~mm}$ thick with a diameter of $25 \mathrm{~mm}$, glued to the bottom of the reaction chamber.

The ultrasonic wave was generated by a Hameg HM 8131-2 arbitrary waveform generator and amplified by a Krohn-Hite Model 7500 amplifier for the sonochemical reaction experiments and a LeCroy WaveSurfer 452 oscilloscope to read-out PMT measurements.

The powers used for the experiments were calculated from calorimetric measurements with a Hanna K-type Thermocouple leading to $12.7 \mathrm{~W}$ for the high power and $3.32 \mathrm{~W}$ for the low power settings. Since control over the heating of the liquid volume was not available, temperature measurements were carried out before and after irradiation times of 3 minutes at similar conditions at which the SL and SCL signals were recorded. The thermocouple was removed from the liquid chamber during sonication to avoid damaging the thermocouple tip. At low power $(3.32 \mathrm{~W})$ the temperature did not increase by more than $3 \mathrm{~K}$. For high power $(12.7 \mathrm{~W})$ the temperature increase was of $10 \mathrm{~K}$. The $10 \mathrm{~s}$ period would generate an increase in temperature of around $0.6 \mathrm{~K}$. We overestimate the temperature increase to be at least $1 \mathrm{~K}(2 \mathrm{~K}$ maximum) to be conservative. For this reason, the PMT measurements lasted in general no longer than $10 \mathrm{~s}$ to avoid large temperature variations.

The continuously applied acoustic field generated a standing wave depending on the height of the liquid column. For liquid heights close to one-quarter or threequarters of the acoustic wavelength (approximately 250 or $300 \mu \ell$ volume of liquid, respectively) a pressure antinode is expected to be located on the substrate and a node at the free liquid-air interface. In this study frequencies of about $200 \mathrm{kHz}$ with a corresponding water height of approximately $5 \mathrm{~mm}$ were used $(250 \mu \ell)$.

A Hamamatsu E849-35 PMT (2.5 ns risetime), with $15 \mathrm{~mm}$ diameter glass window, amplified by a Canberra H.V. Supply Model 3002 was placed to capture the 
light emitted by the sonicated liquid on top of the chamber.

The experiments were conducted with different liquids but the same volumes $(250 \mu \ell)$ at ambient conditions and open to the atmosphere. The voltage readout of the PMT corresponded to the SL and SCL emission, where applicable, from a certain population of bubbles.

The variations to the bottom surface (square silicon substrate of $10 \mathrm{~mm}$ width) of the micro-sonoreactor (the same as presented in [18]) were: blank with no pit (equivalent to a conventional US reactor of the bath type), one, two or three pits (small predefined cylindrical crevices on the silicon surface). The pits acted as nucleation sites for microbubble streamers that would otherwise not be present at the conditions studied. Details of the manufacturing process of the silicon substrate can be found in Chapter 3, Section 3.2.1.

Two different power settings were chosen out of the three presented in [18], corresponding to lower and higher power levels. The main reason to select these two settings is that they evidence a clear difference in the bubble pattern and the ultrasonic power being supplied to the whole system. Hence, we expect to see differences in the bubble populations capable of emitting light and producing radicals in all cases.

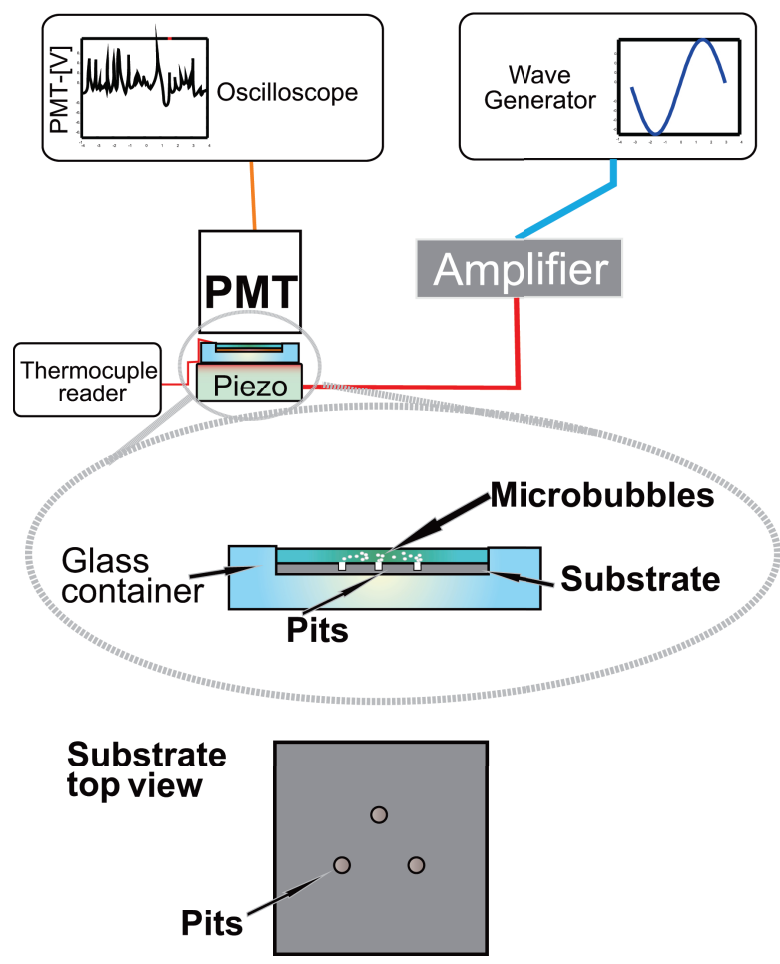

Figure 4.1: Experimental setup (not to scale) showing the different components used. The zoomed inset shows the microreactor and a top view of a three-pit substrate. 
The controlled and localized acoustic microbubble generation can be sustained for at least several hours due to dissolved gas in the liquid transported into the pit by a process similar to rectified diffusion [4, 41-43]. Since temperature and gas escaping the microchamber could not be controlled in this particular case, all experiments were carried out within 5 to 10 minutes.

For the SCL imaging, a digital SLR camera (Nikon D90) with 18-55 mm AFS zoom lens using the settings ISO1250, f 5.0 and a $60 \mathrm{~s}$ exposure time.

For SL imaging a NF Multifunction synthesizer WF-1946A with a NF HSA-4014 amplifier, and a fan to cool the microreactor were used to get similar conditions as described before. The exposure time for experimental imaging and dark conditions subtraction was 10 minutes with a BitranBS-41L cooled CCD camera coupled to a Nikkor $35 \mathrm{~mm}$ lens and a magnifying glass.

\subsection{Results and Discussion}

Contrary to the highly reproducible characteristics of single bubble cavitation, multiple bubbles are difficult to characterize since the bubble size distribution is constantly changing and bubbles do not cavitate always in phase with the driving frequency (period doubling and chaotic behaviors are reported in the literature [44, 45]). However, the overall multiple bubble activity can be quantified by measuring the total SL intensity. Figure 4.2 shows PMT output recorded for $10 \mathrm{~s}$ for the three systems studied. Despite the appearance of emission spikes (common for these systems [21, 46]), the average intensity of each system was used for comparison in the following discussion.

The emission spikes along a constant low intensity emission are short pulses that originate from specific bubbles that, upon reaching an appropriate size in the expansion phase, collapse and emit a strong light pulse. Micro-shocks occurring within the bubble during final collapse stages are reported elsewhere both for water and luminol solutions [46, 47].

It can be seen in Figure 4.2 that the emission spikes mainly appear for the water and luminol system and not that frequently for the aqueous propanol system. Despite the less frequent presence of these spikes in the propanol system, the average intensity was higher than that observed for water. This behavior provides evidence that the bubble population is largely transient as there is no SL quenching [39, 48, 49]. The presence of propanol can increase the bubble population by lowering the surface tension and facilitaing the pinch-off events of microbubbles from the pit. The white space in between the signal and the "x-axis" for propanol and luminol, not present in plain water, is due to a higher overall SL and SCL intensity. A more detailed analysis for each system is presented in the following sections. 

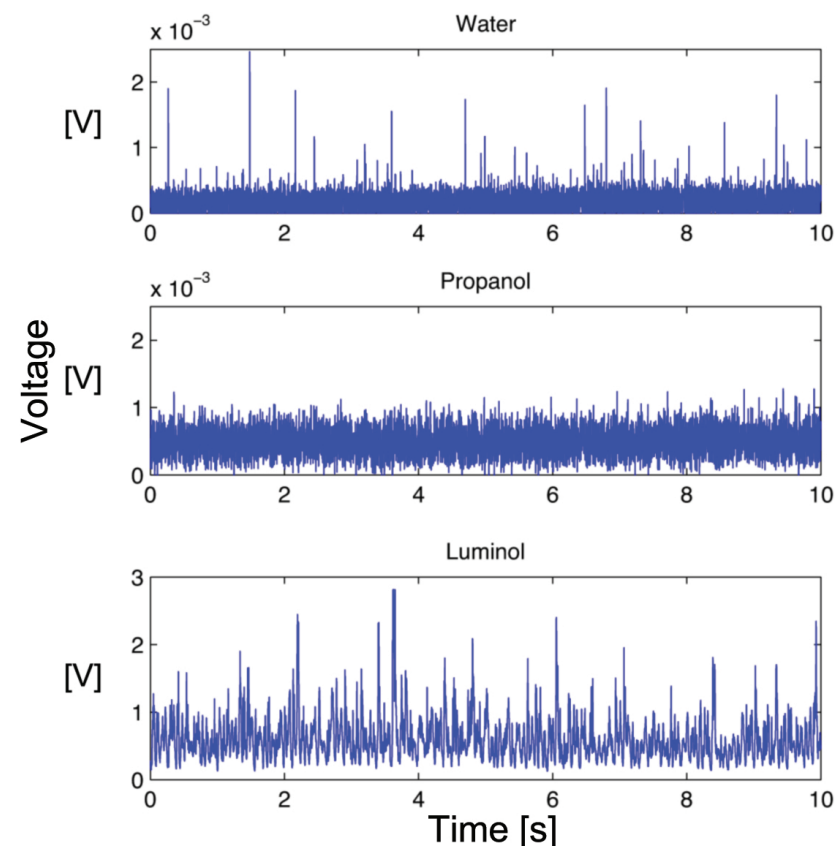

Figure 4.2: Typical emission profiles recorded with the PMT for the different studied experimental conditions.

\subsubsection{Water}

When water is poured over the silicon substrate and US is switched on, a cloud of bubbles appears from the micropits at low power levels. An interesting behavior is observed when there are multiple pits driven at high power: the ejected bubbles travel to a common center point (see Figure 4.3 and supporting videos) due to a complex interplay of primary and secondary Bjerknes forces [18].

Figure 4.4 shows a clear trend of increasing SL intensity with increasing number of pits, both at the low and high power regimes.

As the number of pits is increased, the number of SL active bubbles increases. The increase is almost double in the case of one-pit compared with the blank substrate. For two-pits, the SL enhancement is tripled, whilst for three-pits the SL is almost four times that of the blank substrate at high power. While a similar increase is observed at the higher power level, the relative increase with three-pits compared to two-pits is low. This might be due to stronger bubble clustering effects which are known to reduce the maximum expansion radius and shorten the bubble collapse duration [50].

When trying to image SL in the experiments with air-saturated Milli-Q water and the conditions described up to this point, not enough signal-to-noise images could be 


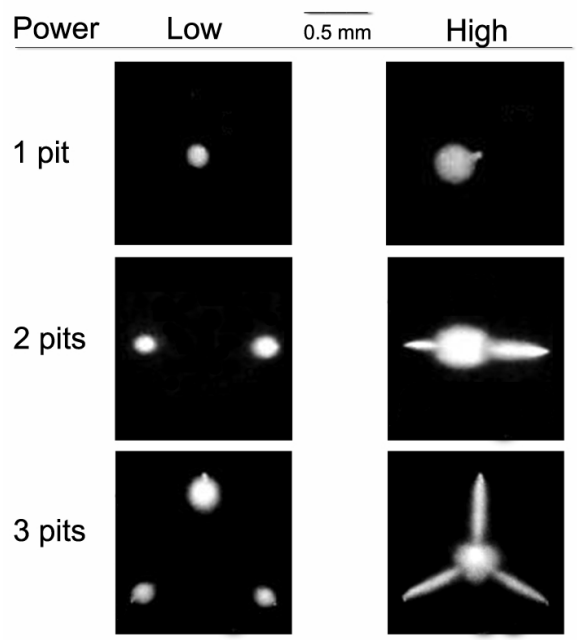

Figure 4.3: Top view of the visible bubble streamers for the different scenarios studied. See Supporting videos

obtained. For this reason Milli-Q water was saturated with argon (Ar) and a glass slide was placed on top of the microreactor to avoid evaporation (see supporting video). During the imaging period the cooling of the piezo was carried out with a fan. The long exposure times required to obtain images like the ones presented in Figure 4.5 made it very difficult to cover the same experimental conditions as for the rest of this work.

Nevertheless, these results allow us to conclude that the SL signal detected with the PMT was primarily due to light emission from the bubbles ejected from the micropits and not from random cavitation events in the bulk liquid.

\subsubsection{Aqueous propanol solution}

The visible bubble pattern for aqueous propanol solutions had no evident change when compared to water; however, as presented in Figure 4.2. spikes present in the water emission profile are not as frequent in propanol solutions and the spike height is on average lower. The average intensities for propanol solutions are presented in Figure 4.6 .

Propanol can cause two effects in sonicated liquids: by adsorbing to the bubble solution interface it hinders bubble coalescence. And due to its volatile nature, it evaporates into the bubble and lowers the polytropic exponent, resulting in less heating inside the bubble [49]. These two effects can affect the SL in two ways. The hindrance to bubble coalescence has been shown to increase the number of transient cavitation bubbles [39]. The volatile nature leads to significant SL quenching in sta- 


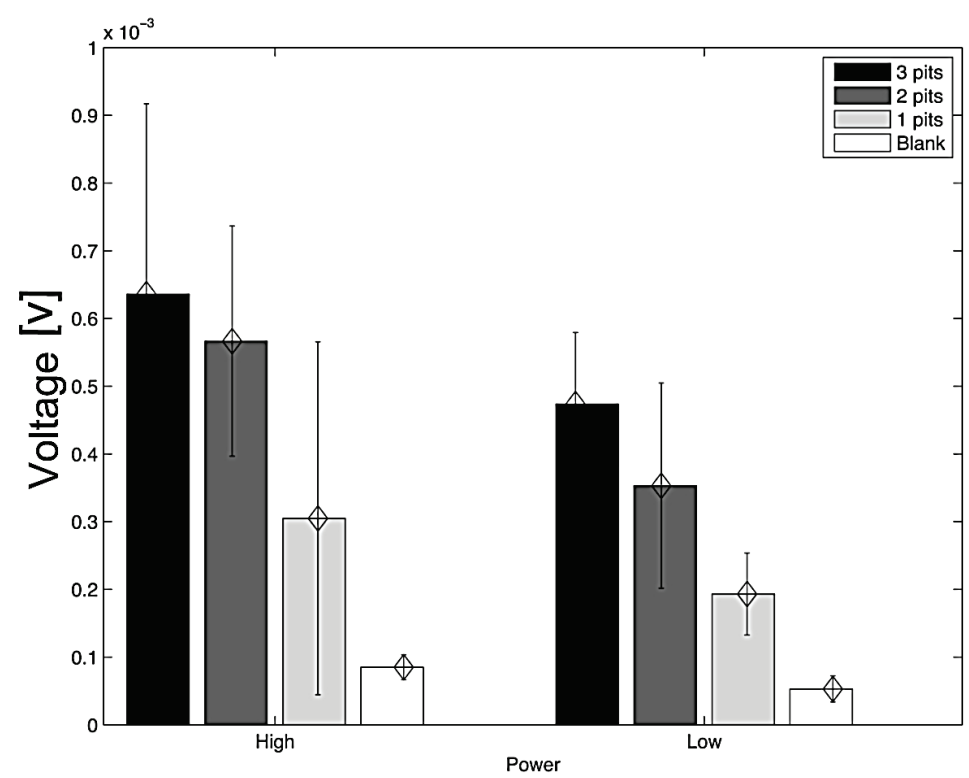

Figure 4.4: Averaged SL intensities for the different studied experimental conditions in water.

ble cavitation bubbles [51]. However, both processes need sufficient time for the propanol to accumulate at the bubble interface. Here, under the conditions of transient cavitation this time is not given. The observation that the average SL intensity for the propanol system instead of quenching the SL signal is higher than that observed in water is in agreement with the fact that the cavitation bubbles generated in the microreactor are transient in nature. As suggested earlier, the lower surface tension of the bubble stabilized on the pit due to the presence of propanol might yield a higher number of bubble streamers generated that in turn contribute to the increase in SL signal. This needs to be supported by future experiments with fast imaging of all these conditions.

\subsubsection{Luminol solution}

Similar bubble streamer patterns from the luminol emission photographs are obtained (Figure 4.7) to those observed in Figure 4.3. The light emitted was bright enough to be seen with the naked eye in adjusted dark conditions.

As can be seen in these images, the SCL and hence radical formation is intensified at the location of the micropits. The average SCL intensities for the case of luminol solutions were several orders of magnitude higher than those from the SL in water as presented in Figure 4.8. It is surprising then that the total emissions arising from the substrates containing pits are more or less identical to those arising from the 


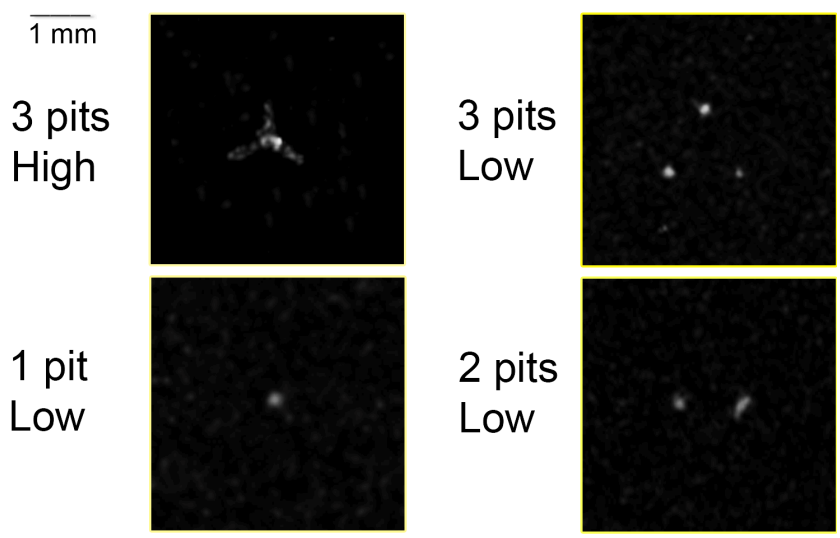

Figure 4.5: SL image from bubble streamers for different scenarios. The top row corresponds to three-pit cases with similar pattern as observed under visible conditions for high and low power. The remaining figures correspond to one-, two-pit cases at low power

blank substrate at low power. For the higher power, the two and three pits systems actually produce a lower yield than the blank substrate. It has been reported before that luminol SCL can have a unusual dependence with increasing power, sometimes reaching saturation and complete fading of intensity [46].

An explanation for our observations is that by driving the systems with two or more pits at high power, the shape of the bubbles in the cluster become deformed. This, combined with liquid flow inside the microreactor chamber, mixing, free liquidair interface oscillation and temperature increase in less than $5 \mathrm{~min}$ can bring a change in the SCL.

We speculate that the observation of different SCL signal trends when compared to our previous study measuring $\mathrm{OH}$ radicals [18] are due to the surface oscillation taking place at the liquid-air interface of the microreactor at the higher power conditions as reported before [52]. More details will be provided in the coming section 4.3.4 and also in the supplementary videos.

The pixel intensities of the luminol photographs were also averaged in time and are presented in Figure 4.9. It can be seen that the two different experimental techniques (PMT and photographic imaging) produced similar trends (compare Figure 4.8 and 4.9 . From the photographic images taken, it can be seen that luminol emission is intensified not just at the location of the pits, but also at the edges of the substrate and other locations like cracks on the silicon substrate edge or the corners of the microchamber which can act as nucleation sites. Such effects occur across all the experiments and will be additive to the luminol emission from the pits. The PMT used to measure the SCL yield picks up light not just from the pits but also from the other places described. 


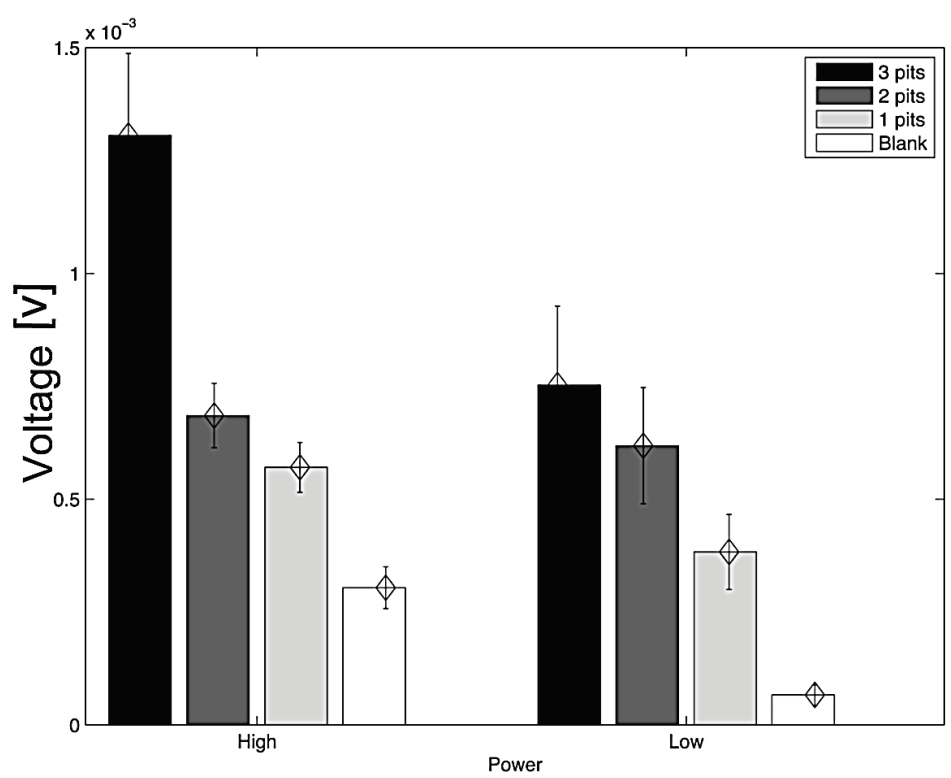

Figure 4.6: Averaged SL intensities for the different studied experimental conditions with aqueous propanol solutions.

\subsubsection{Comparing SL and SCL}

In Figure 4.10 the relative increase of the SL intensity in water and propanol solutions is compared. Note that for all situations there is an increase in SL intensity of propanol over water as described before. For two- and one-pit cases the low power shows a higher relative value than at high power (when the bubble streamers travel parallel to the wall and towards the center point) where the bubble population vary as the bubble clouds change their shape.

When comparing the relative increase in SCL intensity of luminol over the SL signal from water, Figure 4.11 is obtained. Interestingly again the relative intensity is higher for the low power than for high power (now for the two- and three-pit systems) in line with our previous findings that higher power can be detrimental to $\mathrm{OH}$ radical formation [18]. This can be linked with the fact that at low power the population of smaller and more spherical bubbles is larger, and it is expected that these are the ones contributing the most to radical production. Another possibility according to numerical simulations is that $\mathrm{OH}$ production rate decreases at a too high temperature inside an air bubble as $\mathrm{OH}$ is consumed by oxidizing nitrogen inside a bubble [53]. This may suggest that on average, the temperature inside the bubbles is higher with pits compared to the blank conditions. Additionally, the bubble temperature is sometimes increased by the bubble-bubble interaction with smaller bubbles [54]. In the situation with pits, smaller and larger bubbles may result in higher bubble tem- 


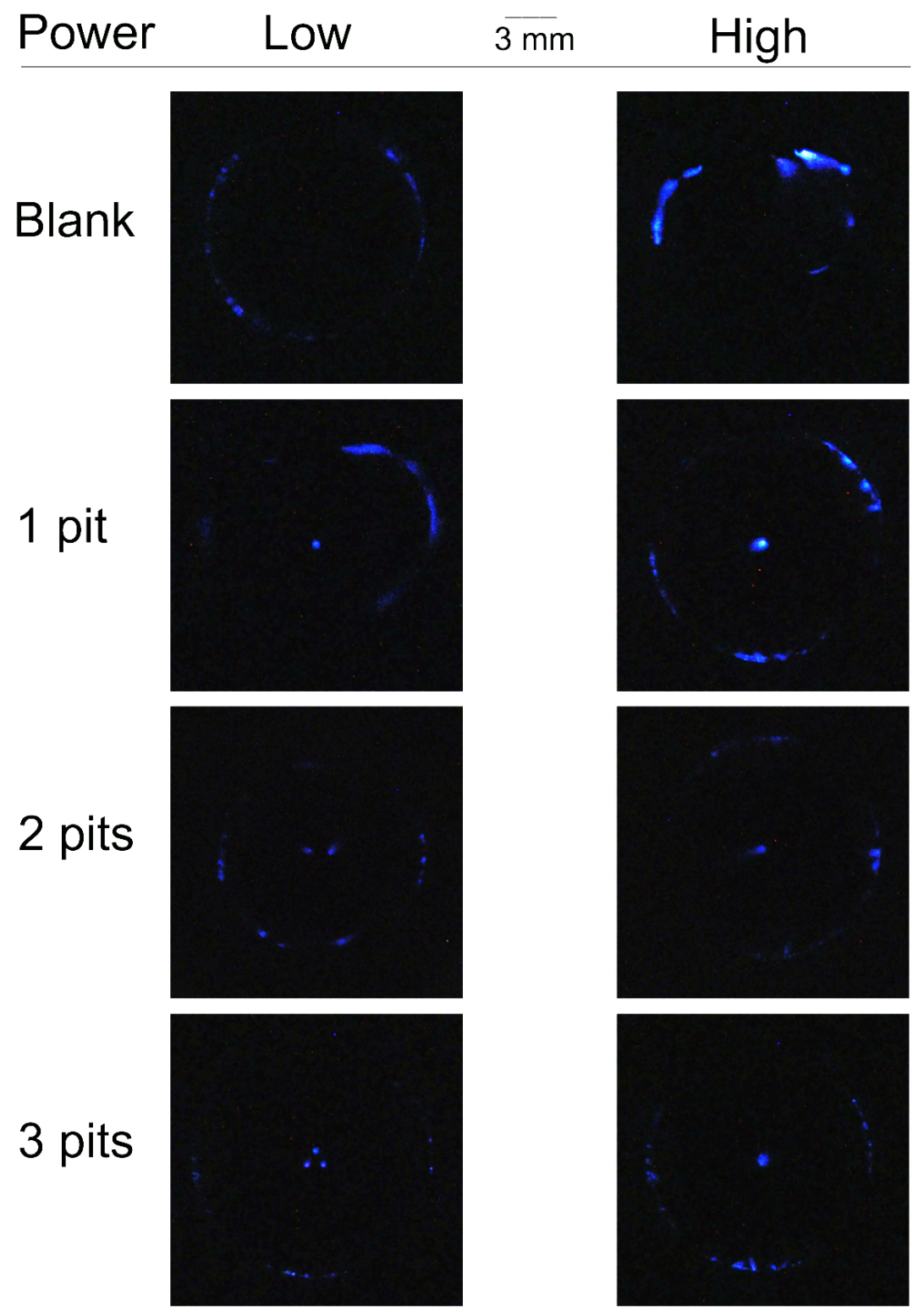

Figure 4.7: Luminol solution images showing sonochemical active regions in white for the different scenarios studied. 


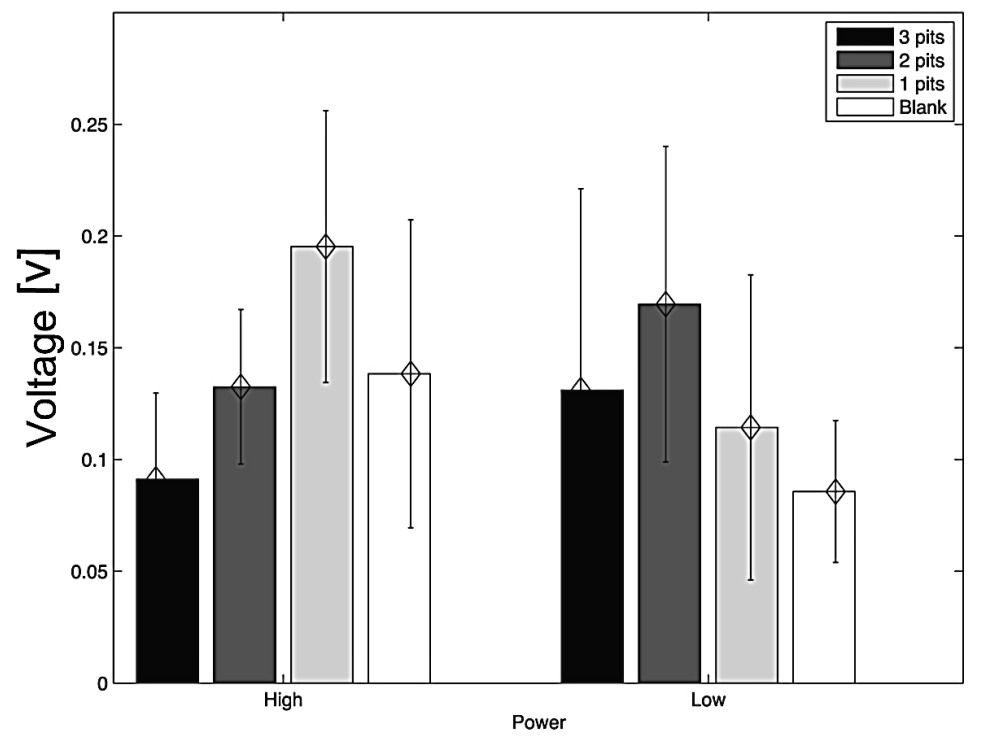

Figure 4.8: Averaged SCL intensities for the different experimental conditions for aqueous luminol solution.

perature inside larger bubbles compared to the case of no pit (blank) when there are mainly tiny bubbles (their size is too small to be recorded by the cameras).

Comparing Figures 4.10 and 4.11 it can be seen that the blank case is a clear indication of the strong influence of the contribution from the bubbles generated at the pits. For the case of one pit it can be observed that there is almost no change in the relative intensity value for both the propanol and luminol when increasing power. This strongly evidences that the interaction of the microbubble streamers generated by more than one pit is an important factor in the trends observed (cluster-cluster interactions through shockwave emission).

To partially illustrate the complexity of this system the influence of bubble-bubble and bubble-boundary interactions on the observed SL should be considered. When microbubbles are exposed to an acoustic field, if smaller than resonant size, they tend to travel to the pressure antinodes and cluster by virtue of Bjerknes forces. This behavior may influence the generated SL. An example is the case of high power for the two- and three-pit systems, where there is a change in the microbubble pattern when compared to the one-pit and blank configuration. A bubble cloud reflects and absorbs the sound field such that a lower intensity will be experienced by bubbles inside the cloud due to shielding. This reduces the intensity of the bubble collapse and the active bubble population, leading to a reduced SL intensity when compared to conventional multibubble cavitation [55]. As mentioned above bubble collapse in 


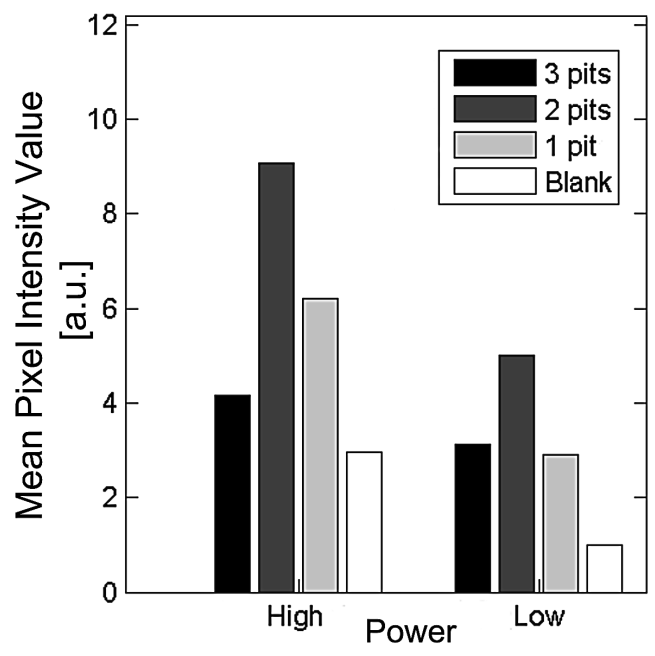

Figure 4.9: Averaged pixel intensity values from the luminol images recorded (SCL) for the different experimental conditions for aqueous luminol solutions. Besides the blue light coming from the center of the silicon substrate (microbubbles generated from the pits) in some cases some SCL signal is seen coming from the edges of the substrate; presumably from crevices existing in the glass reaction chamber or substrate edges.

clusters also results in smaller expansion maximum radius and shorter collapse times [50].

It has been modeled and experimentally confirmed for a single bubble that the strength of the bubble collapse is affected by its translational movement (accelerated due to added mass forces while the driving pressure increases), and that the strength of the bubble collapse and its sphericity (i.e., the focusing power) are key ingredients determining the SL and SCL intensity [4, 56-58]. Indeed, in our experiments higher $\mathrm{SL}$ is observed for higher power.

Referring back to Figure 4.3. At high power, for the two- and three-pit cases (two last figures in the right column of Figure 4.3, we see bubble clustering in between the pits, leading to a depletion of bubbles directly above the respective pits. Therefore, in these cases, the bubbles are actually only cavitating in a thin liquid layer of a width of about $200 \mu \mathrm{m}$ above the surface [35]. Consequently, there is then less mutual shielding of the bubbles as compared to the other four cases (one-pit case at both powers and two- and three-pit cases at low powers). Additionally, at higher power there will be bubbles that expand to a larger size than at lower power, resulting in an increase in SL. We also expect that shockwave emissions from bubble clusters and cluster-cluster interactions (among microbubble streamers generated at each pit) influence the overall SL and SCL in terms of bubble maximum radius and collapse time as has been demonstrated before [50]. 


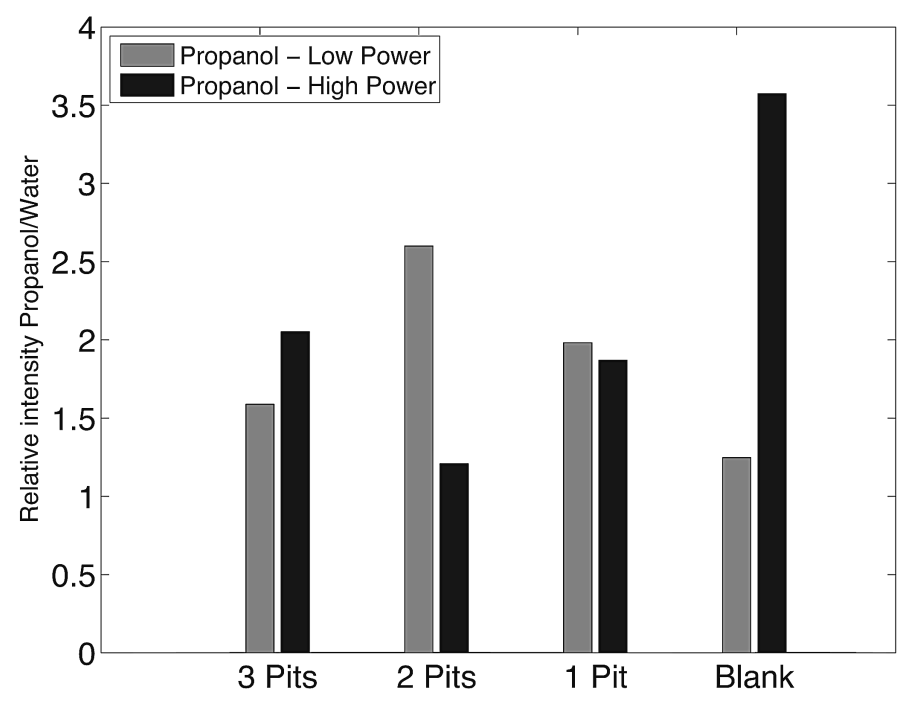

Figure 4.10: Averaged SL intensities for the different experimental conditions for propanol solutions relative to water.

Observations at the highest power give evidence that water can occasionally splash out from the liquid-air interface due to acoustic radiation force (see supplementary video). This has been reported to decrease the efficiency of sonochemical reactions [52], consistent with the reduced SCL signal at high power and regimes with more than one pit. As the meniscus shakes vigorously (a sizzling sound accompanies this process), the cavitation field changes considerably in a way difficult to quantify or predict. This can introduce sources of errors in the SL and SCL measurements. In addition, at the highest power heating and degassing of the liquid occur faster and this obviously changes the conditions for SL and SCL.

It would be interesting to correlate the observed SL profile measured in this study to the influence of fluid mechanics such as flow due to acoustic streaming, microstreaming by meniscus oscillation, microbubbles flowing at different regimes, the interplay of secondary Bjerknes forces and bubble cluster interaction with the overall flow. Other factors such as closing the system with the presence of a glass slide on top of the microreactor to minimize liquid splashing, are among several other conditions that could be investigated but the appearance of degassing bubbles on the glass surface might be an undesired side-effect (see supplementary video). The above mentioned factors would influence the light emission, both from SL and SCL, at the different powers. As a last effort, continuous recording of these bubble streamers (in water, propanol and luminol solutions) may provide further clues to better understand our findings. 


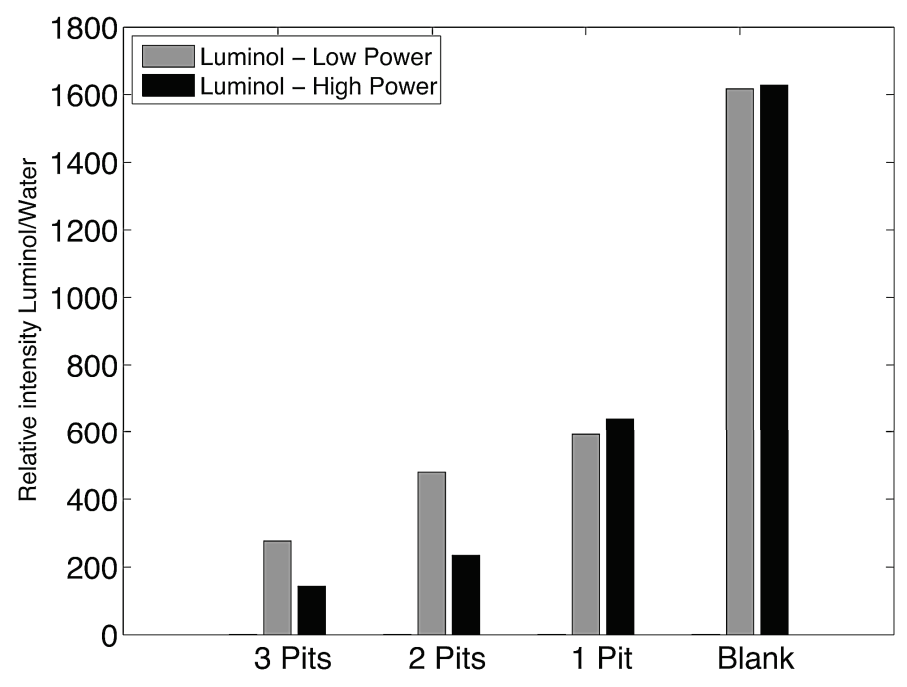

Figure 4.11: Averaged SCL and SL intensities for the different experimental conditions for luminol solutions relative to water.

\subsection{Conclusions}

We have measured light emission at a single frequency from different systems at two different power levels in the presence and absence of pits on surfaces. These pits promoted nucleation of microbubble streamers with distinctive streaming patterns at each power level. SL and SCL imaging provided evidence that the microbubbles arising from the pits are responsible for most of the light emission detected with PMT measurements. The presence of transient cavitation conditions was verified by measuring the SL intensity in propanol solutions. A difference in the light intensity of SL and SCL also led to the conclusion that there is a difference in the bubble population able to emit light and those which are chemically active. The multiple factors affecting the bubble streamers behavior are difficult to resolve individually. For that reason, there exists significant scope for future studies, particularly involving the control of the free surface liquid-air meniscus.

\section{Acknowledgements}

We thank Prof. Franz Grieser and Prof. Andrea Prosperetti for useful discussions and Stefan Schlautmann for technical assistance. This research was supported by the Technology Foundation STW, Applied Science Division of NWO and the Technology Programme of the Ministry of Economic Affairs, The Netherlands. The authors acknowledge financial support from the ARC, Australia. 


\section{References}

[1] M. Ashokkumar, J. Lee, S. Kentish, and F. Grieser, "Bubbles in an acoustic field: an overview", Ultrason. Sonochem., 14, 470-475 (2007).

[2] D. Lohse, "Sonoluminescence-Cavitation hots up", Nature 434, 33-34 (2005).

[3] Y. T. Didenko and K. S. Suslick, "The energy efficiency of formation of photons, radicals and ions during single-bubble cavitation”, Nature 418, 394-397 (2002).

[4] M. P. Brenner, S. Hilgenfeldt, and D. Lohse, "Single bubble sonoluminescence", Rev. Mod. Phys. 74, 425-484 (2002).

[5] W. Lauterborn and T. Kurz, "Physics of bubble oscillations”, Rep. Prog. Phys. 73, 106501 (2010).

[6] M. Marinesco and J. Trillat, “Action des ultrasons sur les plaques photographiques”, Compt. Rend. 196, 858860 (1933).

[7] H. Frenzel and H. Schultes, "Luminescenz im ultraschallbeschickten wasser”, Z. Phys. Chem. B2742 (1934).

[8] L. Crum, "Sonoluminescence, sonochemistry, and sonophysics”, J. Acoust. Soc. Am. 95, 559 (1994).

[9] E. Flint and K. Suslick, “The temperature of cavitation”, Science 253, 1397 (1991).

[10] T. Matula and R. Roy, "Comparisons of sonoluminescence from single-bubbles and cavitation fields: bridging the gap", Ultrason. Sonochem. 4, 61-64 (1997).

[11] K. S. W.B. McNamara III, Y. Didenko, "Effect of noble gases on sonoluminescence temperatures during multibubble cavitation”, Phys. Rev. Lett. 84, 777-780 (2000).

[12] K. Suslick, Y. Didenko, M. Fang, T. Hyeon, K. Kolbeck, W. McNamara, M. Mdleleni, and M. Wong, “Acoustic cavitation and its chemical consequences", Philos. T. R. Soc. A 357, 335 (1999).

[13] K. S. Suslick and D. J. Flannigan, "Inside a collapsing bubble: Sonoluminescence and the conditions during cavitation”, Annu. Rev. Phys. Chem. 59, 659-683 (2008).

[14] A. Brotchie, F. Grieser, and M. Ashokkumar, "Effect of power and frequency on bubble-size distributions in acoustic cavitation", Phys. Rev. Lett. 102, 84302 (2009).

[15] K. Yasui, T. Tuziuti, J. Lee, T. Kozuka, A. Towata, and Y. Iida, "The range of ambient radius for an active bubble in sonoluminescence and sonochemical reactions”, J. Chem. Phys. 128, 184705 (2008).

[16] D. Sunartio, K. Yasui, T. Tuziuti, T. Kozuka, Y. Iida, M. Ashokkumar, and F. Grieser, "Correlation between Na* emission and "chemically active" acoustic cavitation bubbles", ChemPhysChem 8, 2331-2335 (2007).

[17] T. L. N.V. Dezhkunov, "Study into correlation between ultrasonic capillary effect and sonoluminescence", J. Eng. Phys. Thermophys. 77, 53-51 (2004).

[18] D. Fernandez Rivas, A. Prosperetti, A. G. Zijlstra, D. Lohse, and H. J. G. E. Gardeniers, "Efficient sonochemistry through microbubbles generated with micromachined surfaces", Angew. Chem. Int. Ed 49, 9699-9701 (2010).

[19] Y. Iida, K. Yasui, T. Tuziuti, M. Sivakumar, and Y. Endo, "Ultrasonic cavitation in microspace", Chem. Commun. 20, 2280-2281 (2004).

[20] J. Lee, T. Tuziuti, K. Yasui, S. Kentish, F. Grieser, M. Ashokkumar, and Y. Iida, "Influence of surface-active solutes on the coalescence, clustering, and fragmentation of acoustic bubbles confined in a microspace", J. Phys Chem. C 111, 19015-19023 (2007). 
[21] Tandiono, S. Ohl, D. Ow, E. Klaseboer, V. Wong, R. Dumke, and C. Ohl, "Sonochemistry and sonoluminescence in microfluidics", Proc. Natl. Acad. Sci. USA 108, 5996-5998 (2011).

[22] N. Bremond, M. Arora, C. D. Ohl, and D. Lohse, “Controlled multi-bubble surface cavitation”, Phys. Rev. Lett. 96, 224501 (2006).

[23] N. Bremond, M. Arora, S. M. Dammer, and D. Lohse, "Interaction of cavitation bubbles on a wall", Phys. Fluids 18, 121505 (2006).

[24] P. Marmottant, J. P. Raven, H. Gardeniers, J. G. Bomer, and S. Hilgenfeldt, "Microfluidics with ultrasounddriven bubbles", J. Fluid Mech. 568, 109-118 (2006).

[25] B. M. Borkent, S. Gekle, A. Prosperetti, and D. Lohse, "Nucleation threshold and deactivation mechanisms of nanoscopic cavitation nuclei”, Phys. Fluids 21, 102003 (2009).

[26] P. C. G. Cravotto, "Harnessing mechanochemical effects with ultrasound-induced reactions", Chem. Sci. 2, 295-307 (2012).

[27] A. Cobley, L. Edgar, M. Goosey, R. Kellner, and T. J. Mason, "Initial studies into the use of ultrasound to reduce process temperatures and chemical usage in the pcb desmear process", Circuit World 1, 15-23 (2011).

[28] V. D. N.V. Dezhkunov, "Physical backgrounds for application of power ultrasound in fluorescent dye penetrant inspection", in Proc. of $4^{\text {th }}$ European Congress on Acoustics, L231-L234 (Budapest) (2005).

[29] C.-D. Ohl and B. Wolfrum, "Detachment and sonoporation of adherent hela-cells by shock wave-induced cavitation", Bba-Gen Subjects 1-3, 131-138 (2003).

[30] R. Dijkink, S. Le Gac, E. Nijhuis, A. van den Berg, A. Vermes, A. Poot, and C. D. Ohl, "Controlled cavitationcell interaction: trans-membrane transport and viability studies", Phys. Med. Biol. 2, 375 (2008).

[31] M. Ashokkumar, J. Lee, Y. Iida, K. Yasui, T. Kozuka, T. Tuziuti, and A. Towata, "Spatial distribution of acoustic cavitation bubbles at different ultrasound frequencies", ChemPhysChem 11, 1680-1684 (2010).

[32] S. Hilgenfeldt, S. Grossmann, and D. Lohse, "A simple explanation of light emission in sonoluminescence", Nature 398, 402-405 (1999).

[33] S. Luther, R. Mettin, P. Koch, and W. Lauterborn, "Observation of acoustic cavitation bubbles at 2250 frames per second”, Ultrason. Sonochem. 8, 159-162 (2001).

[34] N. Tsochatzidis, P. Guiraud, A. Wilhelm, and H. Delmas, "Determination of velocity, size and concentration of ultrasonic cavitation bubbles by the phase-doppler technique”, Chem. Eng. Sci. 56, 1831-1840 (2001).

[35] D. Fernandez Rivas, L. Stricker, A. G. Zijlstra, H. J. G. E. Gardeniers, D. Lohse, and A. Prosperetti, "U1trasound artificially nucleated bubbles and their sonochemical radical production", Ultrason. Sonochem. Accepted (2012).

[36] S. Labouret and J. Frohly, "Bubble size distribution estimation via void rate dissipation in gas saturated liquid. application to ultrasonic cavitation bubble fields", Eur. Phys. J. Appl. Phys. 19, 39-54 (2002).

[37] W. Chen, T. Matula, and L. Crum, "The disappearance of ultrasound contrast bubbles: observations of bubble dissolution and cavitation nucleation”, Ultrasound Med. Biol. 28, 793-803 (2002).

[38] M. Ashokkumar, J. Lee, Y. Iida, K. Yasui, T. Kozuka, T. Tuziuti, and A. Towata, "The detection and control of stable and transient acoustic cavitation bubbles", Phys. Chem. Chem. Phys. 11, 10118-10121 (2009).

[39] G. Price, M. Ashokkumar, and F. Grieser, "Sonoluminescence quenching of organic compounds in aqueous solution: Frequency effects and implications for sonochemistry", J. Am. Chem. Soc., 126, 2755-2762 (2004).

[40] R. Toegel, B. Gompf, R. Pecha, and D. Lohse, "Does water vapor prevent upscaling sonoluminescence?", Phys. Rev. Lett. 85, 3165-3168 (2000). 
[41] R. E. Apfel, "Role of impurities in cavitation threshold determination", J. Acoust. Soc. Am. 48, 1179-1186 (1970).

[42] L. Crum, "Nucleation and stabilization of microbubbles in liquids", Appl. Sci. Res. 38, 101-115 (1982).

[43] J. B. Fowlkes and L. A. Crum, "Cavitation threshold measurements for microsecond length pulses of ultrasound”, J. Acoust. Soc. Am. 83, 2190-2201 (1988).

[44] W. Lauterborn and E. Cramer, "Subharmonic route to chaos observed in acoustics", Phys. Rev. Lett. 47, 14451448 (1981)

[45] C. Cabeza, A. Sicardi-Schifino, C. Negreira, and G. Montaldo, "Experimental detection of a subharmonic route to chaos in acoustic cavitation through the tuning of a piezoelectric cavity", J. Acoust. Soc. Am. 103, 3227 (1998)

[46] K. Negishi, "Experimental studies on sonoluminescence and ultrasonic cavitation", J. Phys. Soc. Jpn. 16, 1450-1465 (1961).

[47] P. Jarman, "Sonoluminescence: a discussion”, J. Acoust. Soc. Am. 32, 1459 (1960).

[48] J. Lee, S. Kentish, and M. Ashokkumar, "The effect of surface-active solutes on bubble coalescence in the presence of ultrasound”, J. Phys. Chem. B 109, 5095-5099 (2005).

[49] R. Toegel, S. Hilgenfeldt, and D. Lohse, "Suppressing dissociation in sonoluminescing bubbles: The effect of excluded volume”, Phys. Rev. Lett. 88, 034301 (2002).

[50] K. Yasui, Y. Iida, T. Tuziuti, T. Kozuka, and A. Towata, "Strongly interacting bubbles under an ultrasonic horn", Phys. Rev. E 77, 016609 (2008).

[51] J. Guan and T. Matula, "Time scales for quenching single-bubble sonoluminescence in the presence of alcohols”, J. Phys. Chem. B 107, 8917-8921 (2003).

[52] T. Tuziuti, K. Yasui, T. Kozuka, and A. Towata, "Influence of liquid-surface vibration on sonochemiluminescence intensity", J. Phys. Chem. A 114, 7321-7325 (2010).

[53] K. Yasui, T. Tuziuti, and Y. Iida, "Optimum bubble temperature for the sonochemical production of oxidants", Ultrasonics 42, 579-584 (2004).

[54] K. Yasui, A. Towata, T. Tuziuti, T. Kozuka, and K. Kato, "Effect of static pressure on acoustic energy radiated by cavitation bubbles in viscous liquids under ultrasound”, J. Acoust. Soc. Am. 130, 3233-3242 (2011).

[55] Z. Zeravcic, D. Lohse, and W. van Saarloos, "Collective oscillations in bubble clouds", J. Fluid Mech. 680, 114-149 (2011).

[56] M. P. Brenner, D. Lohse, and T. F. Dupont, "Bubble shape oscillations and the onset of sonoluminescence", Phys. Rev. Lett. 75, 954-957 (1995).

[57] R. Sadighi-Bonabi, R. Rezaei-Nasirabad, and Z. Galavani, "The dependence of the moving sonoluminescing bubble trajectory on the driving pressure”, J. Acoust. Soc. Am. 126, 2266 (2009).

[58] S. Hatanaka, H. Mitome, K. Yasui, and S. Hayashi, "Single-bubble sonochemiluminescence in aqueous luminol solutions", J. Am. Chem. Soc. 124, 10250-10251 (2002). 


\title{
5 Enhancing Acoustic Cavitation using Artificial Crevice Bubbles *
}

\begin{abstract}
We explore the rich behavior displayed when pre-defined cavitation nuclei are driven continuously in the $\mathrm{kHz}$ regime $(80,99.5$ and $200 \mathrm{kHz})$. The nuclei consist of stabilized gaspockets in cylindrical micropits of $30 \mu \mathrm{m}$ diameter etched in silicon or glass substrates. It is found that above an acoustic pressure threshold the behavior of the liquid-gas meniscus switches from a stable drum-like vibration to expansion and deformation, frequently resulting in microbubble pinch-off. Just above this threshold small bubbles are continuously and intermittently ejected. At elevated acoustic pressures the pinch-off mechanism becomes more pronounced and cavitation bubble clouds are formed which remain in the vicinity of the micropit bubble. Surprisingly, the resulting loss of gas typically does not lead to deactivation of the pit which can be explained by gas rectified diffusion processes.
\end{abstract}

${ }^{*}$ To be submitted as: Aaldert Zijlstra, David Fernández Rivas, Han J.G.E. Gardeniers and Detlef Lohse, Enhancing acoustic cavitation using artificial crevice bubbles, to J. Ac. Soc. Am. 2012 


\subsection{Introduction}

Most applications based on acoustic cavitation, such as ultrasonic cleaners, emulsifiers or sonochemical reactors, rely on the spontaneous and seemingly random appearance of cavitation bubbles [1-3]. The subsequently generated cavitation state, meaning the bubble sizes, locations, dynamics and so on, are notoriously difficult to describe and even more difficult to accurately predict or control. This is mainly due to the inherent multiparameter nature of cavitation and its complex dependence on the liquid and acoustic parameters. Manufacturers and researchers therefore have to resort to empirical methods to optimize the desired mechanical or chemical effects induced by acoustic cavitation bubbles [4-6]. The resultant of these effects is not only determined by the magnitude of the response of each individual bubble, but also by the total number of bubbles and their locations. The increase of cavitation activity would seem just a matter of increasing the acoustic amplitude. However, due to effects such as acoustic shielding and bubble-bubble interactions, this may not necessarily lead to an increase in efficiency [7--9] (Chapters 3 and 4). On the other hand, there is also a frequency dependent lower acoustic power threshold for cavitation activity, usually referred to as the nucleation threshold [10]. Hence in the situations where lower acoustic amplitudes are required, e.g. to prevent heating or surface damage [11], it might not even be possible to generate or sustain a sufficient bubble population.

Enhancing cavitation bubble mediated effects may be achieved by actively regulating not only the acoustic amplitude, but also the bubble population. Therefore, an ideal solution is to introduce microbubbles of the required size into the liquid at the required locations. For example, various studies have shown that the presence of $u l$ trasound contrast agents - microbubbles stabilized by an external shell - significantly enhances cavitation related effects [12, 13]. Other methods such as bubble injection using gas flow through membranes [14], or flow focusing devices [15], might also be beneficial for increasing bubble populations in acoustic cavitation.

Here we present results of a different method to locally enhance acoustic cavitation activity in a continuous ultrasound pressure field. Our method consists of introducing microbubbles into the liquid which are entrapped and stabilized in micromachined well defined cylindrical crevices, an example of which is shown in Figure 5.1. These bubbles are essentially artificial versions of naturally occurring cavitation nuclei better known as "crevice bubbles" [16, 17]. Bubble formation from crevice bubbles occur, not only in cavitation inception, but also in supersaturated liquids such as carbonated beverages [18, 19], and in liquid pool boiling [20]. The use of artificial crevice bubbles was motivated by earlier studies of Bremond et al. [21, 22] and Borkent et al. [23] where the response of the entrapped bubble to a single shock wave was investigated. In this chapter we investigate the dynamics of stabilized cylindrical micropit bubbles under continuous ultrasound irradiation. It will be shown that the 
presence of stabilized microbubbles significantly enhances local cavitation activity by bubble pinch-off processes.

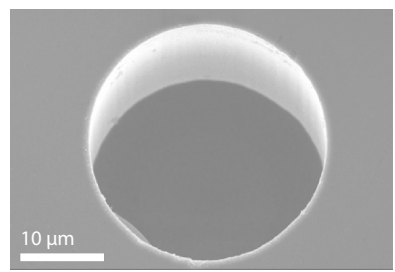

Figure 5.1: SEM image of a micromachined cylindrical cavity of $30 \mu \mathrm{m}$ diameter and $10 \mu \mathrm{m}$ depth in a silicon substrate.

Unlike a spherical bubble which will quickly dissolve under the excess ambient pressure and due to surface tension (also depending on the size and the gas concentration), an entrapped bubble can be in stable mechanical and diffusive equilibrium for an extended period of time [24]. The presence of a crevice bubble nucleus basically constitutes a "weak-spot" in the liquid. Any change in the pressure or gas saturation conditions will cause a volumetric response of the bubble as it seeks to re-establish its equilibrium state. Nucleation is the event where the equilibrium cannot be established and the bubble expands out of the micropit [17, 23]. There are three different but interrelated ways for this to occur. Nucleation can be realized by pressure reduction, but also by an increase in gas saturation or by a rise in temperature.

It is important to make a distinction between nucleation, i.e. the unstable growth of the bubble outside the pit, and a possible bubble pinch-off from the nucleus. Many authors use the term nucleation for the combined process, however the underlying mechanisms are different. Once nucleated, a bubble can detach from the micropit due to effects such as buoyancy, liquid drag or acoustic pressure gradients. Bubble detachment, or pinch-off, is a well studied effect in nucleate boiling [25] and particularly in supersaturated liquids [19], but surprisingly not in acoustic cavitation. Even though bubble detachment from entrapped surface bubbles is normally assumed to occur in acoustic cavitation, to the authors knowledge, there exists few studies in which this is actually reported. In 1962, Hughes \& Nyborg [26] drilled holes of $200 \mu \mathrm{m}$ diameter in a sonotrode tip in order to enhance cavitation effects at reduced acoustic amplitudes. They observed bubble growth, surface instabilities and bubbles leaving the holes but they remained rather speculative on the details of the process. Their method however did result in increased cavitation activity due to the presence of artificial crevice bubbles. Similarly, Howkins [27] studied the erosion on brass surfaces from bubbles nucleated at $20 \mathrm{kHz}$ from a bubble stabilized on a pit of $1 \mathrm{~mm}$ diameter and depth drilled on the sonotrode surface. In degassed water and in the absence of the drilled pit erosion effects were not observed.

Here we are interested in the response of artificial micropit bubbles to a continu- 
ous acoustic field, and how this response can contribute to enhancement of cavitation activity. In the following, the process of bubble nucleation and pinch-off in acoustic cavitation is investigated in more detail using high-speed imaging methods.

\subsection{Materials \& methods}

\subsubsection{Machining of micropits}

In order to obtain micropit bubbles which are comparable in size with naturally occurring crevice bubbles, but large enough to allow visual observations, we chose to create cylindrical pits of $30 \mu \mathrm{m}$ in diameter. The pits were micromachined under clean room conditions both in double-side polished silicon wafers as well as in fused silica glass. The holes were etched into the substrates at the desired depth using a plasma dry-etching machine (Adixen AMS 100 SE Alcatel, process BHARS). The depths were $\sim 30 \mu \mathrm{m}$ and $6 \mu \mathrm{m}$ for the silicon and fused silica glass respectively. The wafers were diced into square pieces of $1 \times 1 \mathrm{~cm}$. We found that a stable liquidgas micromeniscus was always present after immersion of the substrate even though no extra measures were taken to hydrophobize the micropit as was done by e.g. Bremond et al. [21, 22].

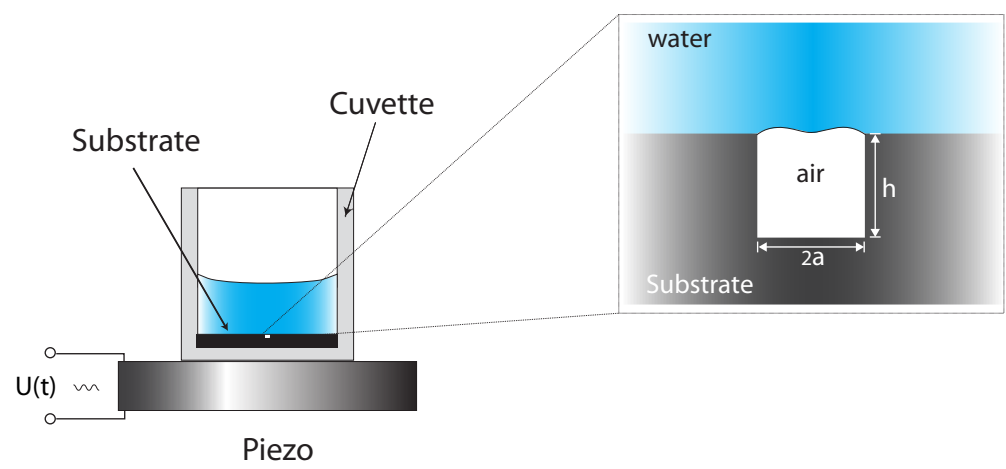

Figure 5.2: Schematic of the piezo-cuvette combination in side view.

\subsubsection{The acoustic field}

In this study, three types of setups were used. The silicon substrates were placed on the bottom of one of two small containers providing stability, liquid containment and convenient optical access. The first type of container was a cubic cuvette, made of optical quality quartz glass with inner dimensions $1 \times 1 \times 1 \mathrm{~cm}$. The second type was cylindrical with an inner diameter of $1.5 \mathrm{~cm}$ and a depth of $3 \mathrm{~mm}$. The acoustic 
field was introduced by means of a piezoelectric element (Ferroperm PZ27, $6 \mathrm{~mm}$ thickness and diameter of $2.5 \mathrm{~cm}$ ) glued to the containers (see Figure 5.2). The maximum response of the piezo and cuvette combination when filled with water was found to occur at frequencies close to $80 \mathrm{kHz}$ and $200 \mathrm{kHz}$ corresponding to radial and thickness vibration modes.

In order to take full advantage of the transparency of the glass substrates with pits, the system described by Tandiono et al. [28] was used. Here, the glass substrates together with four piezo elements were glued to a glass microscope slide. Upon adding demineralized water (left standing for one day to ensure full saturation) onto the substrates, a pocket of air was entrapped within the cylindrical micropits. The presence of the liquid-gas interface could easily be verified using dark-field illumination microscopy.

The piezoelectric elements of the cuvette systems were actuated using a continuous sinusoidal voltage generated by a function generator (model WW1072, Tabor Electronics Ltd.) which was amplified using a wide band amplifier (Krohn-Hite, model 7602). The glass system was actuated by an arbitrary wave generator Agilent 33522A and amplified with an AG series amplifier T\&C Power Conversion. The input voltage and current signal were measured using an oscilloscope (Tektronix, DPO 4043) and a current probe (Tektronix, CTA-2). The small liquid volume and the close proximity to the piezo element hampered reliable pressure measurements, therefore the input electrical power was taken as a relative measure of power -the acoustic power cannot be directly extracted with these values.

Since the applied acoustic field is continuous, a standing wave is formed in the container depending on the shape and dimensions of the liquid volume. It was found that the largest response of the micropit bubble occurred for liquid heights close to one-quarter or three-quarters of the acoustic wavelength. This situation corresponds to a pressure antinode situated on the substrate and a pressure node at the free waterair interface. For the two cuvette systems heights close to $\frac{\lambda}{4}=4.7 \mathrm{~mm}$ for $80 \mathrm{kHz}$ and $\frac{3 \lambda}{4}=5.6 \mathrm{~mm}$ for $200 \mathrm{kHz}$ were set by manual filling. For the transparent glass substrate system, a frequency of $99 \mathrm{kHz}$ was used corresponding to an effective liquid height of approximately $3.8 \mathrm{~mm}$.

\subsubsection{Imaging methods}

The timescale of acoustic cavitation is set by the period of the driving acoustic field. Hence, in order to resolve the dynamics of ultrasonically driven (micropit) bubbles and resulting bubble cloud, high-speed imaging techniques are required. Here we use two types of high-speed cameras which are the Photron, model SA1.1 with framerates up to $500 \mathrm{kfps}$ and the Brandaris 128 capable of frame rates up to $25 \mathrm{Mfps}$ [29]. These cameras are used to capture transient effects. High-speed cameras allow visualization of extremely fast events, however the limited spatial resolution of 
the produced images limits image analysis. Therefore an additional ultrashort single flash imaging method was applied which enabled recording of high resolution images without motion induced blur. The setup used for this particular imaging method is depicted in Figure 5.3. The illumination source consists of a pulsed laser (Nd:YAG, Litron, model Nano S 65-15 PIV, wavelength $532 \mathrm{~nm}$ ) with a pulse duration of $7 \mathrm{~ns}$ (full width at half maximum) and a maximum pulse energy of $65 \mathrm{~mJ}$.

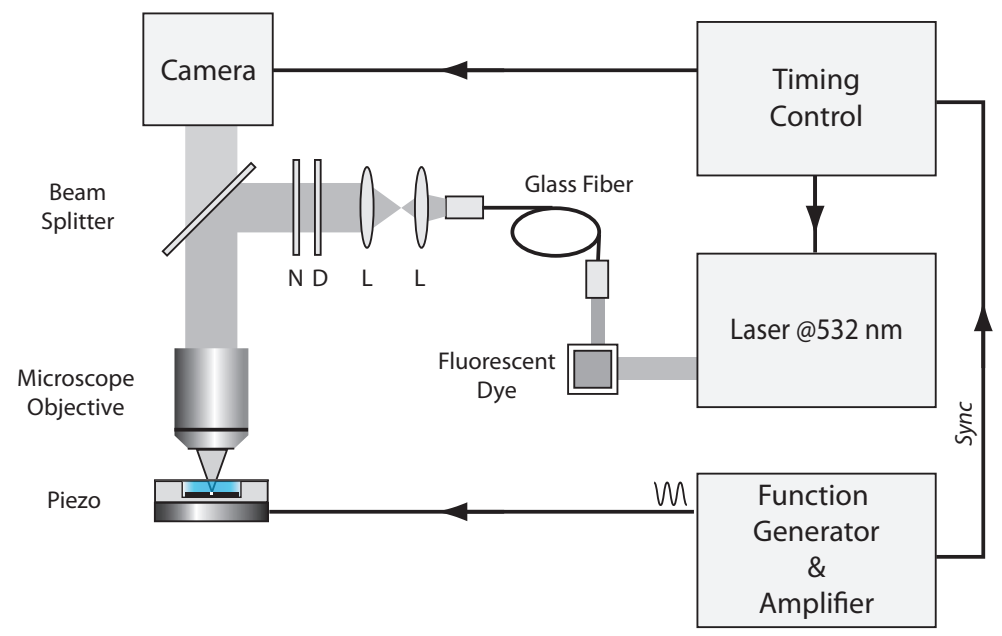

Figure 5.3: Schematic overview of the setup used for single flash imaging of micropit bubble pinch-off and cavitation in top-view. After exiting the fiber the beam is diffused (D) and filtered from any remaining laser light using a notch-filter (N) (Semrock, NF01-532U-25). Illumination of the full field of view is achieved by adjusting the angle of divergence of the beam using two positive lenses $(L)$.

The laser pulse is directed into a quartz cuvette (Hellma Quartz Suprasil with dimensions $10 \times 10 \times 40 \mathrm{~mm}$ ) containing fluorescent laser dye dissolved to saturation in ethanol (LDS 698, Exciton Inc., Dayton, U.S.). The geometry of the cuvette combined with the concentrated dye solution results in high-intensity fluorescence emerging at $90^{\circ}$ with the exciting laser beam [30]. More details of this method are described elsewhere [8, 31]. The fluorescent illumination can be used in back-lighting configuration for side view or, as shown in Figure 5.3, in reflective mode (top view) enabled by the reflectiveness of the silicon substrate. For this purpose the light pulse is relayed using a glass fiber and coupled into the microscope (Olympus). Image magnification was obtained with a long working distance objective (LMPLFLN $10 \times$, Olympus, WD $21 \mathrm{~mm} / \mathrm{NA}=0.25$ or the SMPLFLN $20 \times$, Olympus, WD $25 \mathrm{~mm} / \mathrm{NA}=0.25$ ). The illumination flash was synchronized with the single frame camera (Lumenera LM165, pixel size of $6.45 \times 6.45 \mu \mathrm{m}$, resolution $1392 \times 1040)$ using a pulse delay controller (Berkeley Nucleonics, model 575). Additionally, the delay controller was 
synchronized with the acoustic driving signal.

\subsection{Results}

\subsubsection{Driving at $80 \mathrm{kHz}$}

First we consider the micropit bubble driven by an $80 \mathrm{kHz}$ standing acoustic field. Here the cuvette system was used in order to enable side-view imaging using the microscope and high speed camera (Photron SA1.1) combination. For small driving amplitudes $(\sim 45 \mathrm{~mW})$ there was no visible response of the micropit bubble. For increased acoustic pressures $(>50 \mathrm{~mW})$, however, streams of microbubbles of various sizes could be observed which were ejected intermittently from the micropit bubble. This behavior was found to be reproducible. The character of the pinch-off could be one of two different types, both occurring under similar conditions. For the first type the liquid-gas interface did not extend visibly outside the pit while bursts of microbubbles were expelled upwards, seemingly originating from different locations on the interface (Figure 5.4(a)). The details of the pinch-off in this regime could not be resolved optically. This in contrast with the second type of pinch-off (Figure 5.4(b)), where the micropit bubble expands hemispherically outside the micropit during the low pressure phase of the acoustic field and withdraws again inside the pit when the pressure inverts.
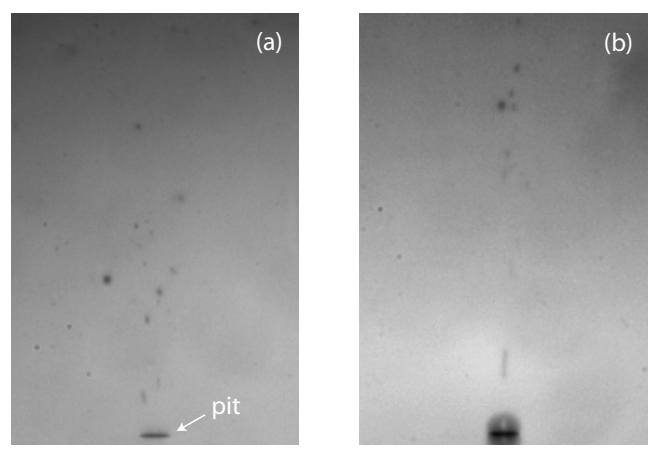

Figure 5.4: Selected frames of a micropit bubble expelling microbubbles. Image (a) shows irregular bubble pinch-off where microbubbles seen as black spots move upward in various angles with the vertical axis. Image (b) shows a regime where the micropit bubble extends outside the pit perimeter and the pinched off bubbles move upwards along the vertical axis.

A sequence of frames of a high-speed movie of a bubble pinch-off event in the second regime is shown in Figure 5.5. Here a microbubble of approximately $5 \mu \mathrm{m}$ in diameter is pinched-off during the collapse phase of the micromeniscus somewhere between $20 \mu \mathrm{s}$ and $22 \mu \mathrm{s}$. Though difficult to distinguish, the interface of the micromeniscus changes from its initial hemispherical shape to a bell-shape during the 
collapse phase. This indicates that the side wall of the meniscus is collapsing before the top wall. Occasionally this radial flow focusing is sufficient to overtake the top part of the interface thereby separating it from the rest of the meniscus. In all of the observed pinch-off events of this type, the bubble separates from the meniscus during the contraction phase, most likely corresponding to the high pressure phase of the acoustic cycle. The resulting stream of microbubbles is visible in Figure 5.4(b). Because of the axial symmetric character of the pinch-off also the trajectories of the bubbles are mainly along the vertical axis.
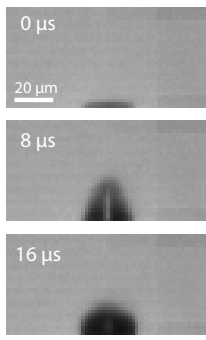

$24 \mu \mathrm{s}$
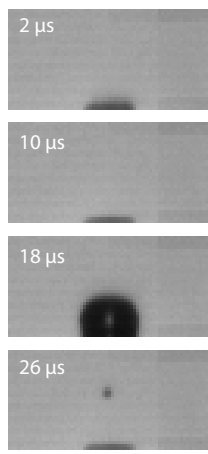
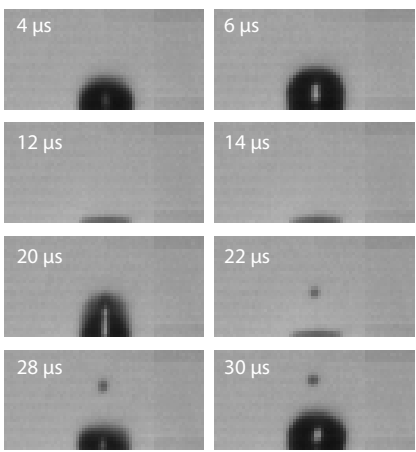

Figure 5.5: Sequence of frames from a $500 \mathrm{kfps}$ high-speed movie showing the response of the micropit bubble during 2.5 acoustic cycles. In the second cycle from 12 to $24 \mu$ s a $5 \mu \mathrm{m}$ diameter microbubble is pinched-off.

What causes these two types of bubble pinch-off mechanism? From the observations we may speculate that the bubbles are generated by a "folding" of the liquidgas interface resulting from large amplitude capillary waves. These closed capillary waves resemble the famous "Crapper wave" solutions [32]. The regime shown in Figure 5.4(a) where bubbles are pinched-off from different locations on the interface would then correspond to a shorter capillary wavelengths compared to the regime shown in Figure 5.4(b) and Figure 5.5.

Estimates of the bubble sizes and pinch-off rates were obtained using image processing techniques implemented in Matlab R2009bß). Two such obtained time traces are shown in Figure 5.6, one for the irregular type pinch-off (a) and one for the regular type pinch-off (b). These traces reveal that the bubble pinch-off processes are quite erratic, both in pinch-off rate, as well as the ejected bubble sizes. At some instances (e.g. near $60 \mathrm{~ms}$ in Figure 5.6(a)) bursts of multiple bubbles (typically with radii less than $5 \mu \mathrm{m}$ ) are expelled while at other moments it can take several milliseconds before the next pinch-off event takes place. The average rate of bubble pinch-off for these particular traces was approximately $2 \mathrm{kHz}$ or once every 40 acoustic cycles. The bubble size distributions (shown in the insets in (a) and (b)) reveal that the majority of the ejected bubbles had radii less than $7 \mu \mathrm{m}$ where the larger bubbles 
typically resulted from bubble coalescence. Surprisingly, the irregular type pinch-off process appears to result in bubbles having radii mostly near $2 \mu \mathrm{m}$ while the regular type shows a broader distribution. Bubble coalescence was however more prevalent in the first type giving rise to larger bubble radii compared to that of the second type, which is also reflected in the bubble distributions. It should be noted that smaller bubbles $(<1.5 \mu \mathrm{m}$ radius) also were observed but limited image resolution prohibited estimation of their radii.
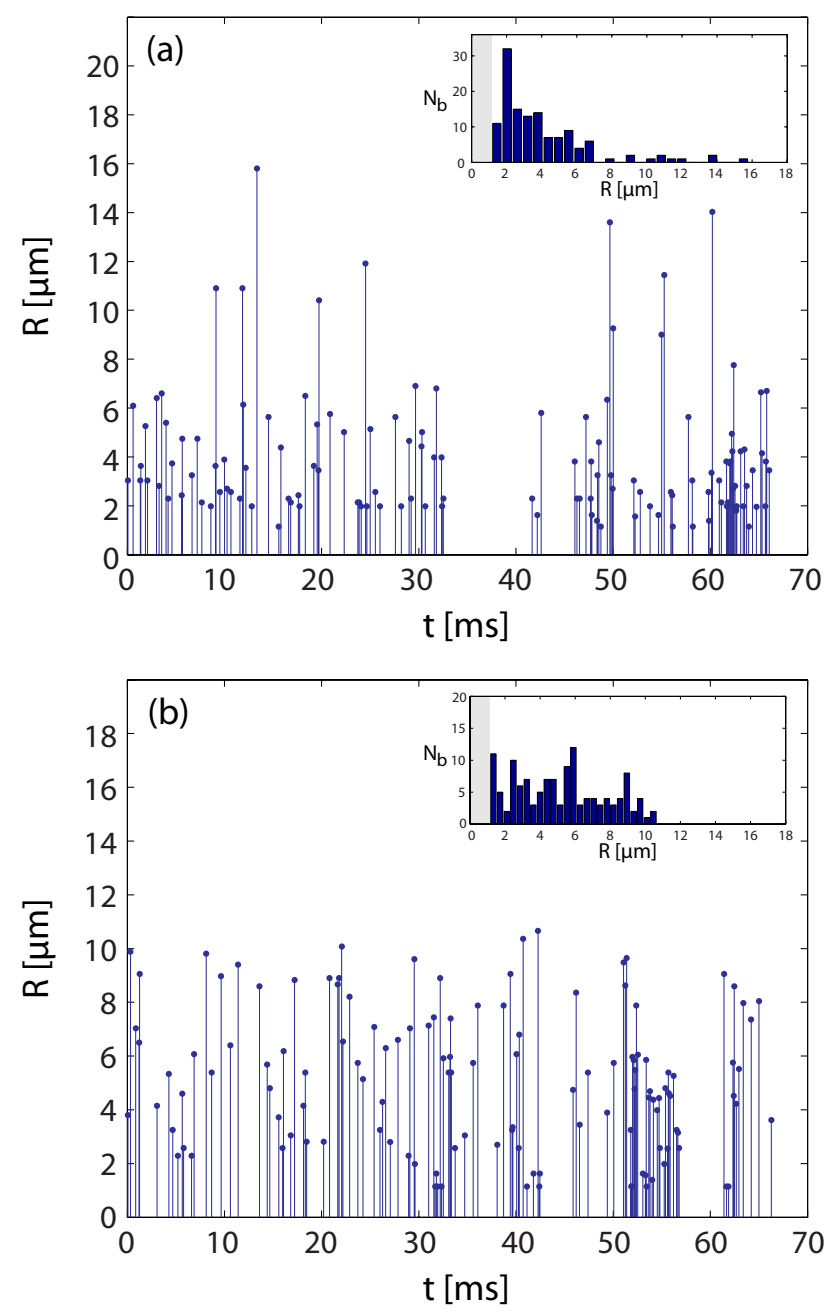

Figure 5.6: Ejected bubble events marked by bubble radius in time for the irregular type pinch-off $(a)$ and regular type pinch-off $(b)$. The insets show the histograms with $N_{b}$ the number of bubbles. The data was obtained from $15 \mathrm{kfps}$ high-speed movie recordings. The image resolution did not allow bubble sizing for radii smaller than $1.3 \mu \mathrm{m}$ (indicated by the gray areas in the distributions). 
All of the observed ejected bubbles have radii smaller than the linear resonance radius of $38 \mu \mathrm{m}[33]$ for this frequency. The size of the micropit bubble on the other hand is closer to its linear resonance radius for this frequency $(98 \mathrm{kHz})[34]$ which may explain the difference in oscillation amplitude. For example, from the RayleighPlesset equation [33] it follows that a spherical bubble with an equilibrium radius of $3 \mu \mathrm{m}$ would expand to a maximum radius of $4 \mu \mathrm{m}$ for an acoustic amplitude of approximately $90 \mathrm{kPa}$. Since such bubble expansions were not observed it follows that this pressure value is a conservative estimate for the lower driving amplitude required for bubble pinch-off as shown in Figures 5.4 and 5.5 .

From the recordings, estimates for the bubble velocities could be obtained. For the regular type of pinch-off it was found that the velocity of the bubbles immediately after pinch-off was typically as high as $2 \mathrm{~m} / \mathrm{s}$ and slowing down to about $50 \mathrm{~mm} / \mathrm{s}$ some $200 \mu \mathrm{m}$ above the substrate. For the irregular type pinch-off the velocities were found to be an order of magnitude lower. The larger number of bubble coalescence events observed in this regime compared to the regular type may be related to these lower velocities.

The most remarkable observation however was that even though the micropit bubble continuously ejected microbubbles, the loss of gas did not result in diminishing or deactivation of the process. The pinch-off rate corresponds to a gas volume flux in the order of $100 \mu \mathrm{m}^{3} / \mathrm{s}$ away from the micropit which must be balanced by an influx of equal magnitude. This transport is most likely of diffusive nature, where a rectifying mechanism may take place, similar to the well known process of rectified diffusion of spherical bubbles [35]. Here, both the area effect and the shell effect may occur. In addition, it is possible that the oscillation-induced microstreaming (see e.g. Miller [36]) draws in liquid from the bulk where the concentration of dissolved air is larger, thereby sustaining the pinch-off process. This same microstreaming could also account for the observed bubble trajectories, possibly in combination with primary and secondary Bjerkness forces.

\subsubsection{Driving at $200 \mathrm{kHz}$}

We now consider the response of the micropit bubble driven at $200 \mathrm{kHz}$. Here the cuvette setup is used again but now imaged in top view (Brandaris 128 high-speed camera). The driving frequency is approximately twice the linear resonance frequency $(98 \mathrm{kHz})$ of the micropit and lies below the second resonance of $243 \mathrm{kHz}$. Similar to what was observed for the $80 \mathrm{kHz}$ driven micropit bubble, there is a threshold for the pressure above which bubble pinch-off occurs. Interestingly, in the regime below this threshold the liquid-gas interface exhibits alternating dark and bright rings which appear to move radially outward periodically with the driving frequency (see Figure 5.7(a)). 

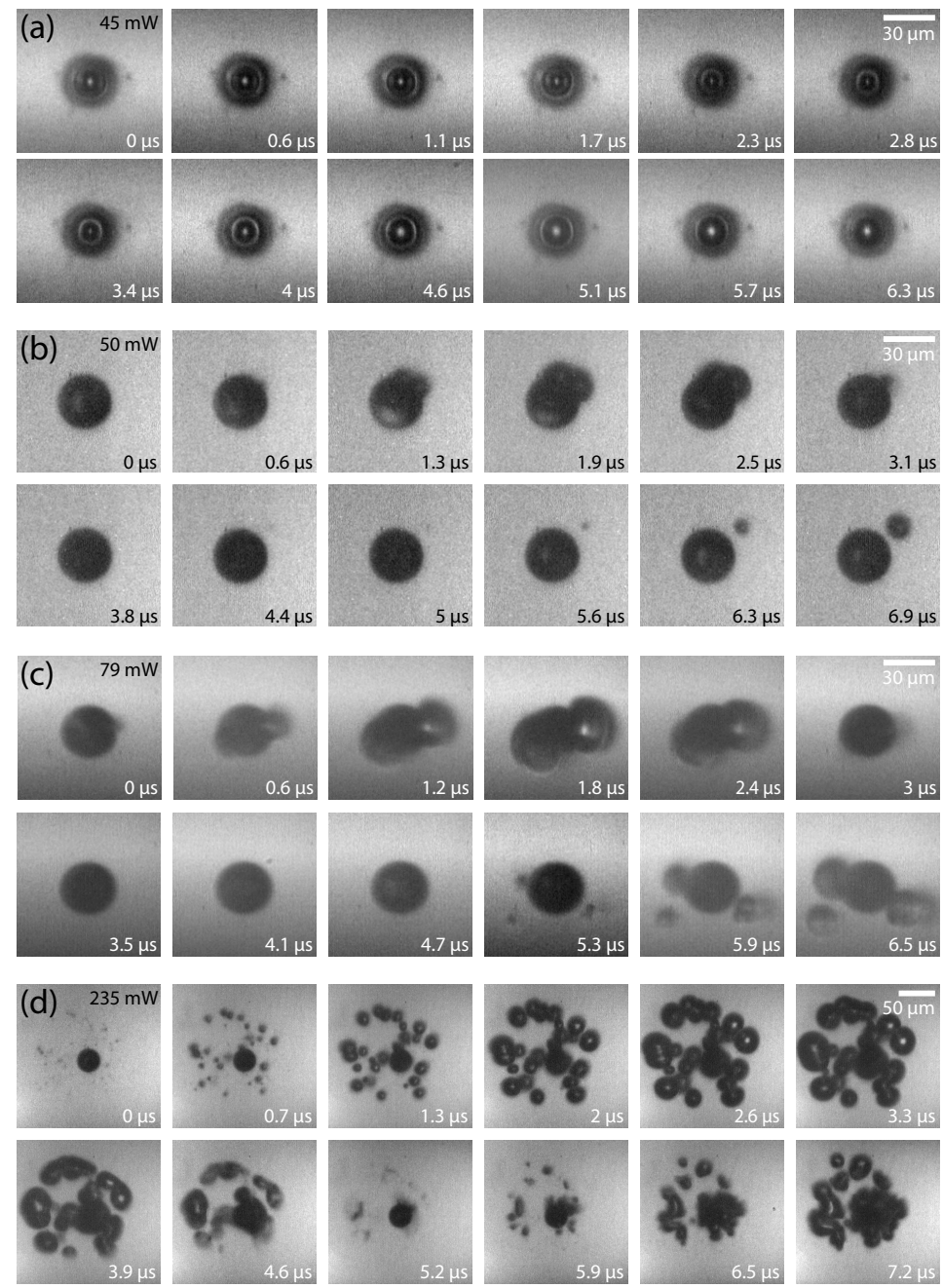

Figure 5.7: Four sequences of frames taken from high-speed recordings in reflective imaging mode (top-view) of the micropit for increasing driving amplitude. Sequence (a) is at approximately $45 \mathrm{~mW}$ showing ring-structures. Increasing the driving power to $50 \mathrm{~mW}$ induces interface deformation and pinch-off $(b)$. Further increase $(79 \mathrm{~mW}$ in $(c)$ and $235 \mathrm{~mW}$ in $(d))$ both increases the bubble number and maximum expansion. Note the different scale in $(d)$.

Since it is difficult to reconstruct the shape of the interface based on the intensity of the reflected illumination it cannot be concluded a priori that these rings are due to surface ripples. Nevertheless, from these images it can be inferred that the interface retains its axial symmetry and oscillates at the same frequency as the driving acoustic field. This symmetry is lost however when the threshold is exceeded. A sequence of frames taken from a high-speed movie is shown in Figure 5.7(b) with driving pressure 
slightly above the threshold, corresponding to an input power of $50 \mathrm{~mW}$. Here the rings are no longer visible and occasionally bubbles are pinched off which in turn respond to the acoustic field by expansion and collapse. In this regime the bubbles that are pinched-off generally are drawn back into the micropit bubble within at most 3 acoustic cycles, most likely due to mutual acoustic forces. In contrast to the $80 \mathrm{kHz}$ driven micropit bubble, the ejected microbubbles do not move upward but remain in close proximity of the substrate. From many similar recordings in this regime it appears that once a bubble is detached from the micropit bubble, a subsequent pinch-off event will not occur as long as the bubble is not recaptured. This may indicate a volume requirement for pinch-off. As shown in Figure 5.7 (c), increasing the amplitude enhances the deformation of the interface and bubbles are continuously pinched-off from the liquid gas interface. Also the number of free bubbles oscillating just outside the pit perimeter increases as well as their maximum expansion size. The bubbles still remain close to the micropit bubble and are observed to oscillate up to 3 or 4 cycles before breaking-up or coalescing with the micropit bubble or other bubbles.

The same trend is observed for larger driving amplitudes; more bubbles are pinched-off expanding to larger volumes with dimensions exceeding the diameter of the micropit. As illustrated in Figure 5.7(d) a cavitation bubble cloud is formed which is centered on the micropit. Here both bubble deformation, coalescence and break-up are more pronounced compared to lower driving conditions (Figure 5.7(b) and (c)). Due to these effects, the bubble population, meaning bubble locations and sizes, changes with every acoustic cycle. The response of an individual bubble in the cloud can therefore only be distinguished and followed in time during part of the expansion phase of the cycle (e.g. from $0.7 \mu \mathrm{s}$ to $3.3 \mu \mathrm{s}$ ).

The increase in the number of bubbles with increasing driving amplitude is shown in the graph of Figure 5.8. The estimates for the number of bubbles were obtained from high-speed recordings at different driving amplitudes. The number of bubbles varies considerably with each acoustic cycle (e.g. compare Figure 5.7 (d) at $t=0.7$ $\mu \mathrm{s}$ and $\mathrm{t}=6.5 \mu \mathrm{s}$ ) resulting in a large variance. Exact bubble counts were difficult to obtain due to limited resolution as well as bubbles which remained undetected when positioned just above the micropit bubble or in contact with other bubbles [8] and Chapter 6 .

The growth of the bubble cloud with increasing amplitude is illustrated in Figure 5.9. The frames in (a) show the scattered light from the bubbles in dark-field illumination mode giving an indication of the extent of the cloud. The corresponding frames in (b) obtained using flash-photography reveal the individual bubbles. From similar images the extent of the bubble cloud was measured (Figure 5.9 (c)). The flattening of this graph suggests that the bubble cloud does not grow indefinitely with increasing power and remains limited to approximately 10 times the pit diameter. 


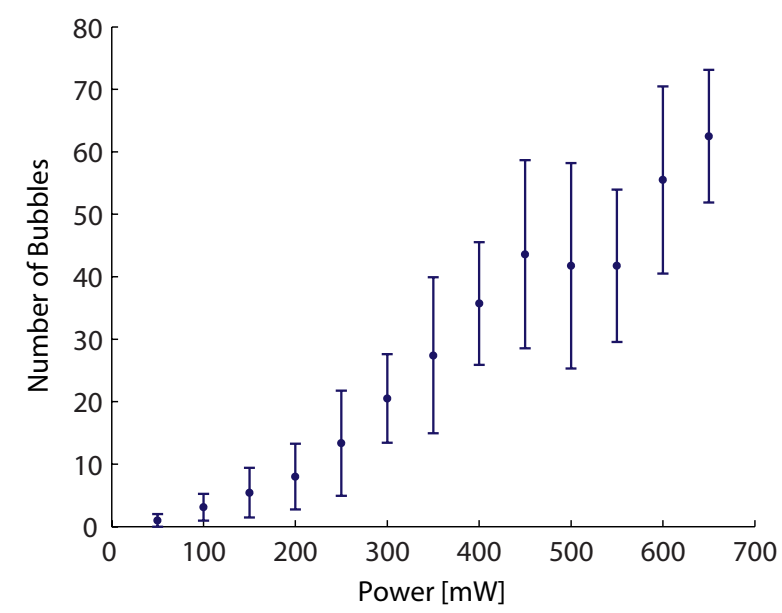

Figure 5.8: Average number of bubbles during one acoustic cycle for different driving amplitudes.

Similar to what was observed at $80 \mathrm{kHz}$, the pit is not deactivated by loss of gas due to pinch-off processes. It was found that the cavitation cloud can remain stable for at least more than an hour, only to disappear when the acoustic field became disrupted due to evaporation of the liquid.

\subsubsection{Driving at $99.5 \mathrm{kHz}$, glass substrates}

The transparency of the setup with the glass substrates enabled improved top-view imaging of the dynamics of the liquid-gas interface in the pit. A typical image sequence of the response of the interface to an $99.5 \mathrm{kHz}$ standing acoustic field obtained with this setup is shown in Fig. 5.10

Following the initiation of the acoustic field at $t=0$ the interface sequentially expands outward and back inward in a drum membrane-like fashion corresponding to the visual changes in brightness (first row). This oscillatory motion was observed to continue for many cycles but occasionally the motion was interrupted as in the 5th frame, during the inward motion, the center of the interface comes into contact with the bottom of the micropit which is shallower than in silicon. It appears that due to this contact the now toroidal interface becomes destabilized and detaches from the top right rim of the micropit $(t=33.3 \mu \mathrm{s})$. In the following cycles the contact-line of the gas volume retracts further until a circular shape is attained $(t=266.7 \mu \mathrm{s})$. Coinciding with this deformation a continuous stream of small bubbles is ejected from the bubble interface along the new axis of symmetry formed by the micropit and the bubble. The bubbles are estimated to have diameters between 1 and $2 \mu \mathrm{m}$ and appear to be ejected once every two acoustic cycles almost parallel to the substrate. 

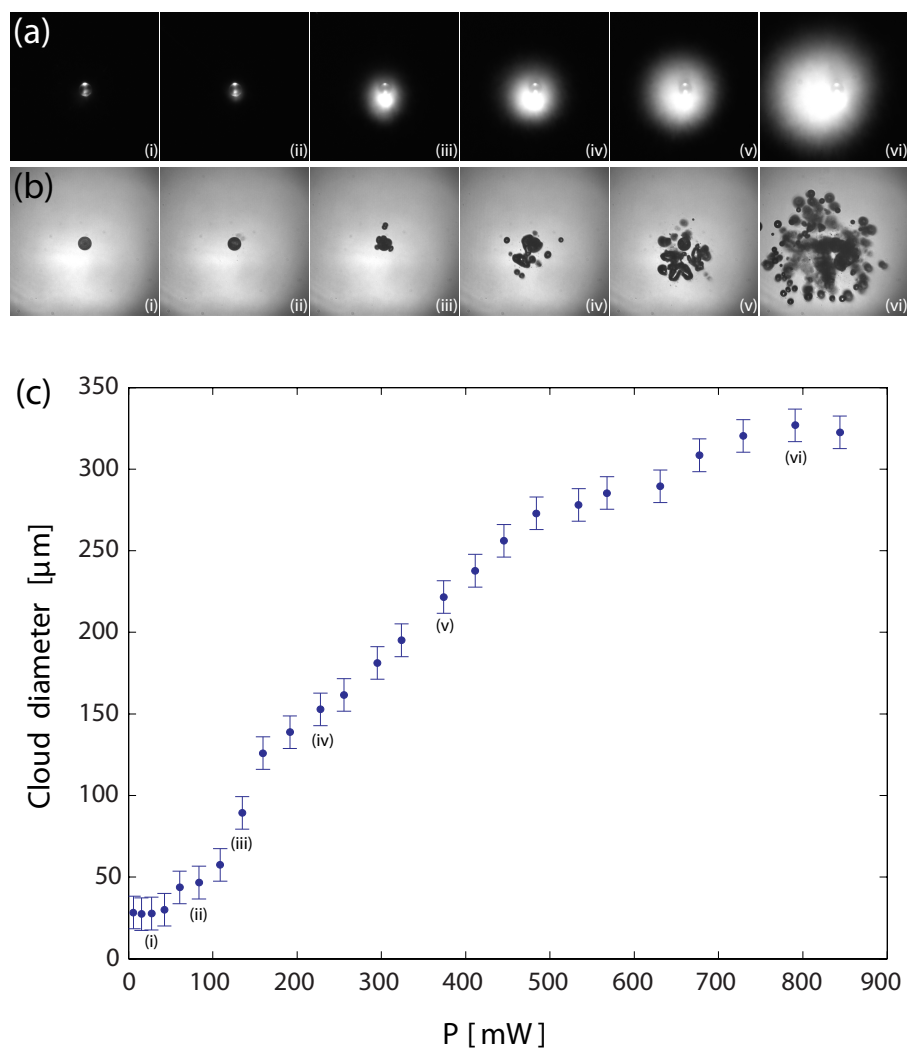

Figure 5.9: (a) Six frames from the $30 \mu \mathrm{m}$ diameter micropit bubble response for increasing driving amplitude obtained with dark field illumination. (b) The corresponding response for the single micropit bubble and its induced cavitation cloud obtained with ultrashort flash photography. (c) Graph of the diameter of the cloud for increasing driving power. The frames (i)-(vi) in (a) and (b) are indicated in the graph.

A clear pinch-off event could not be distinguished from these recordings, however similar to Figure 5.5 the detached bubbles emerge during the contraction phase of the liquid-gas interface.

Once the bubble attains its spherical shape it remains attached for an extended period of time to the lower left corner of the micropit while oscillating with a maximum diameter (in lateral dimensions) of about $20 \mu \mathrm{m}$ (see e.g. $t=288.9 \mu \mathrm{s}$ ). During the contraction phase, the bubble tends to adapt an ovoid or bean-like shape. Occasionally, the bubble contraction occurs faster than the translation of the liquid-gas contact line over the substrate resulting in semicircular bubble extensions (see e.g. $t=272.2 \mu \mathrm{s}$ or $t=2494 \mu \mathrm{s}$ ). At times, this lagging of the interface even resulted in a dual pinch-off from the edges of the bubble $(t=2506 \mu \mathrm{s})$. Another phenomena occurred when larger bubbles were formed either by pinch-off or coalescence $(t=9911$ 


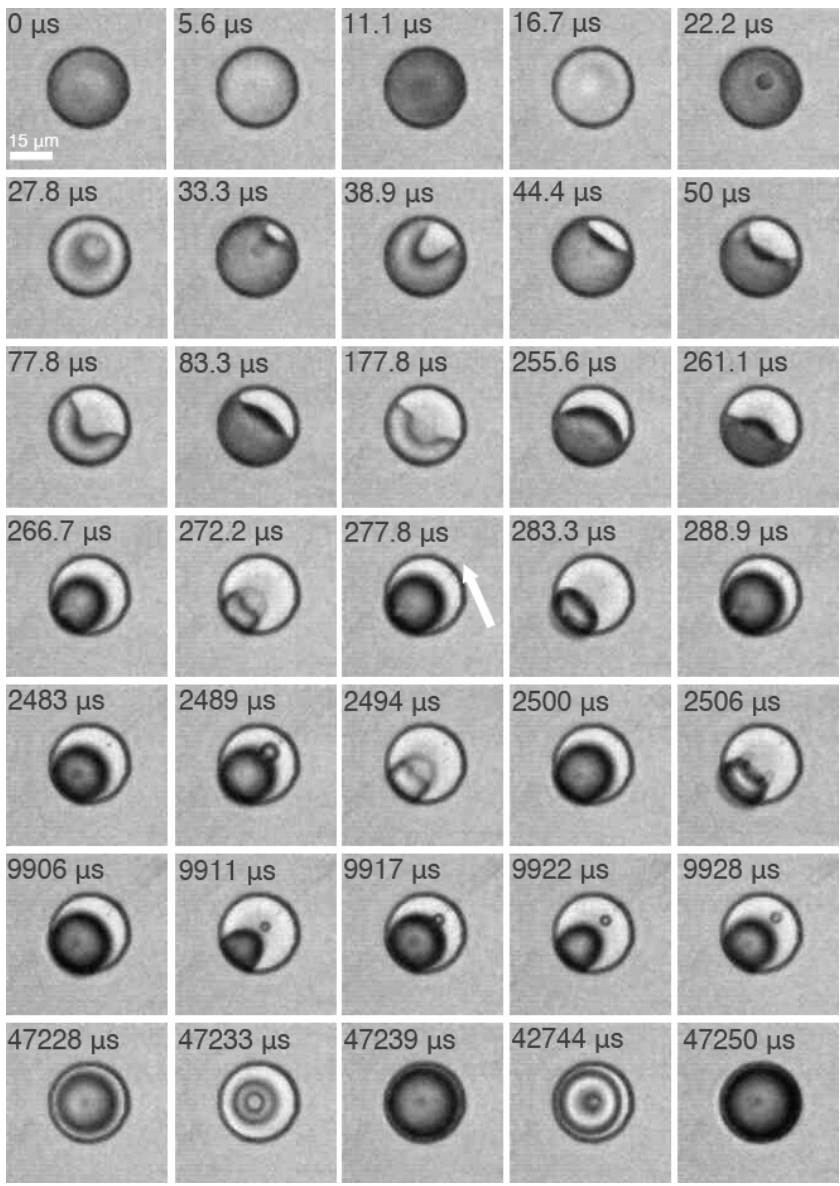

Figure 5.10: Series of frames taken from a high-speed recording (Photron, $180 \mathrm{kfps}, 1 \mu \mathrm{s}$ exposure time) of the glass micropit. The first oscillations occur as a drum membrane until $t=27.8 \mu \mathrm{s}$. The bubble detaches from the rim of the pit and attains a shape close to spherical and stabilizes at one corner of the pit. Subsequent oscillations produce interface deformation and microbubble pinch-off, from more than one location on the bubble surface.

$\mu \mathrm{s})$. These bubbles remained in proximity of the larger bubble, presumably due to secondary Bjerkness forces.

Similar to the previously discussed bubble pinch-off at $80 \mathrm{kHz}$ and $200 \mathrm{kHz}$ from the deeper micropit in silicon, the stabilized and close-to-spherical bubble continuously emits smaller bubbles, but its size does not decrease or dissolves all together. In this case the (horizontal) stream of bubbles ceased when the bubble detached from the lower left corner of the pit and relocated to the center of the micropit $(t=47228$ $\mu \mathrm{s})$. Interestingly the bubble started to oscillate, alternating from spherical to ring or donut shaped (last row of Figure 5.10). 


\subsubsection{Interaction of cloud cavitation}

In the previous section it was shown that in the presence of a single micropit bubble, cavitation activity is initiated and sustained which would not occur without a pit under similar conditions. A natural solution to augment this activity is to introduce additional micropits.

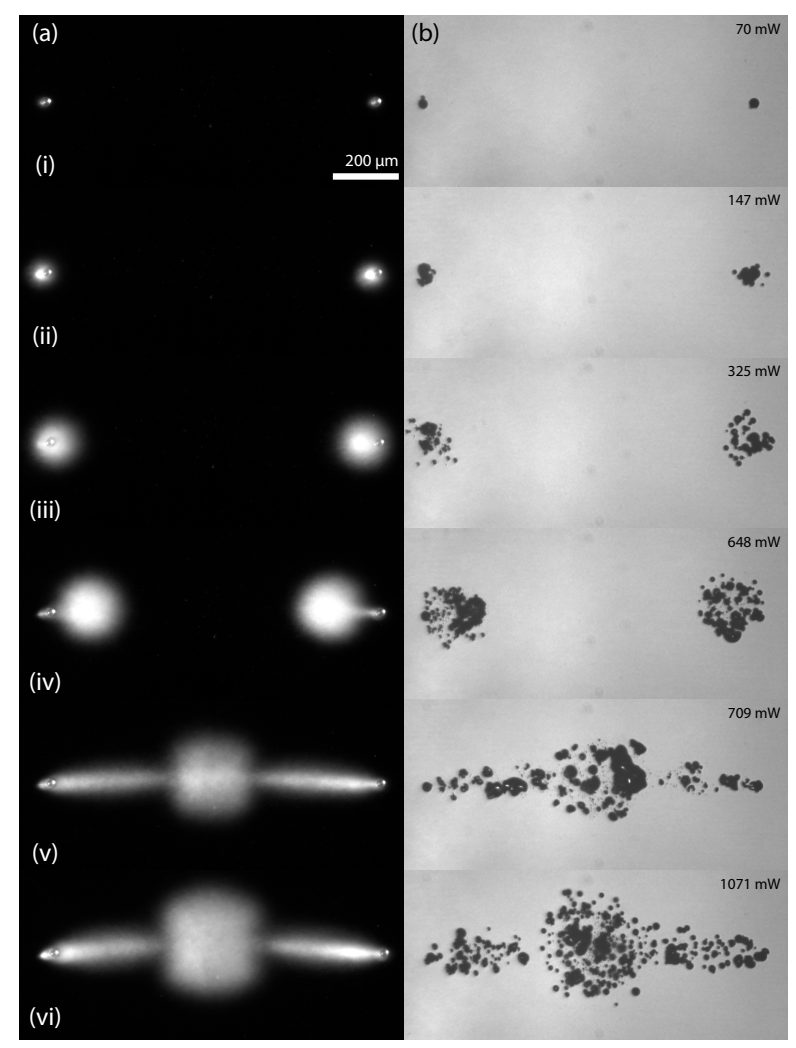

Figure 5.11: The development of the bubble cloud originating from two micropits of $30 \mu \mathrm{m}$ diameter, $1000 \mu \mathrm{m}$ apart. The 6 rows correspond to 6 steps of increasing acoustic amplitude. The left column illustrates the time averaged extent of the cavitation cloud. In the right column the individual bubbles can be distinguished, here taken at approximately maximum expansion.

Indeed, as shown in the images in Figure 5.11 another cavitation bubble cloud is generated from a second micropit. Here the two micropits with $30 \mu \mathrm{m}$ diameter are $1000 \mu \mathrm{m}$ apart. At low driving amplitude the individual clouds appear similar to the single pit system described in the previous section. At elevated powers $(648 \mathrm{~mW})$ however the center of mass of the clouds tends to deviate towards the neighboring micropit (Figure 5.11 (iv)). Above a second pressure threshold the clouds deviate to such an extent that they merge at the midpoint (Figure 5.11 (v) and (vi)). The tran- 
sition between the two states occurs just above $648 \mathrm{~mW}$ driving amplitude (iii) and is quite abrupt, meaning that the intermediate states between (iv) and (v) are either unstable or very sensitive to pressure. At higher amplitude the cloud broadens, similar to the case of a single micropit. Likewise, further increase of driving amplitude does not produce a noticeable expansion of the cavitation cloud. The joining of the clouds results in a peculiar ribbon-like structure with a wider section in the middle measuring $\sim 200 \mu \mathrm{m}$ in diameter.

In order to illustrate the dynamics of the bubbles in the clouds a sequence of frames from a high-speed recording (Brandaris, $1.48 \mathrm{Mfps}$ ) is shown in Figure 5.12. The micropits are located in the lower-right and upper-left corners. The first frame shows the bubble cloud with the bubbles at maximum expansion (similar to the frames (v) and (vi) in Figure 5.11 (b)). During the following cycles (5 $\mu$ s duration) the majority of the bubbles in the cloud collapse and expand again in phase. A few considerably larger bubbles can be observed which, due to their size, exhibit a phase difference with respect to the response of the other bubbles. For example the large bubble close to the lower-right corner continues its collapse until about $4 \mu \mathrm{s}$ (7th frame) while the other bubbles have already started expansion. Similar to the single micropit bubble cloud, the bubbles display considerable coalescence, break-up and deformation, in particular the larger bubbles, probably stimulated by the proximity to the substrate. It is therefore difficult to track individual bubbles in these images. However the recordings do reveal that, on average, bubbles translate after pinch-off from both micropits towards the center of the cloud. In this region a mist of small microbubbles can be observed during the high pressure phase of the acoustic cycle (e.g. at $2 \mu \mathrm{s}$ and $12.2 \mu \mathrm{s}$ ).

These small bubbles may result from increased bubble fragmentation taking place in this region combined with dissolution. Also in this case, the remarkable stability of the cloud suggests that the process is strongly dependent on diffusive transport of dissolved gas. In addition, the recordings shown in Figures 5.9 and 5.11 demonstrate the resilience of the micropit bubbles to dissolve. Evidently, even the strong pressure fluctuations associated with multiple bubble expansions and collapses are insufficient to destabilize the crevice bubble. A more detailed study of these type of bubble phenomena is given in Chapter 6 and elsewhere [8].

\subsubsection{Surface damage}

An interesting finding after several hours of US exposure was a pattern of surface damage on the substrate which was consistent with the region of cavitation activity (Figure 5.13). Cavitation damage is a well known phenomenon [37, 38] and is an illustration of the enormous forces which can be exerted by cavitation bubbles onto solid surfaces. The cumulative effect of multiple bubble collapses result in material fatigue and eventually damage. The SEM-images (done with a High Resolution 

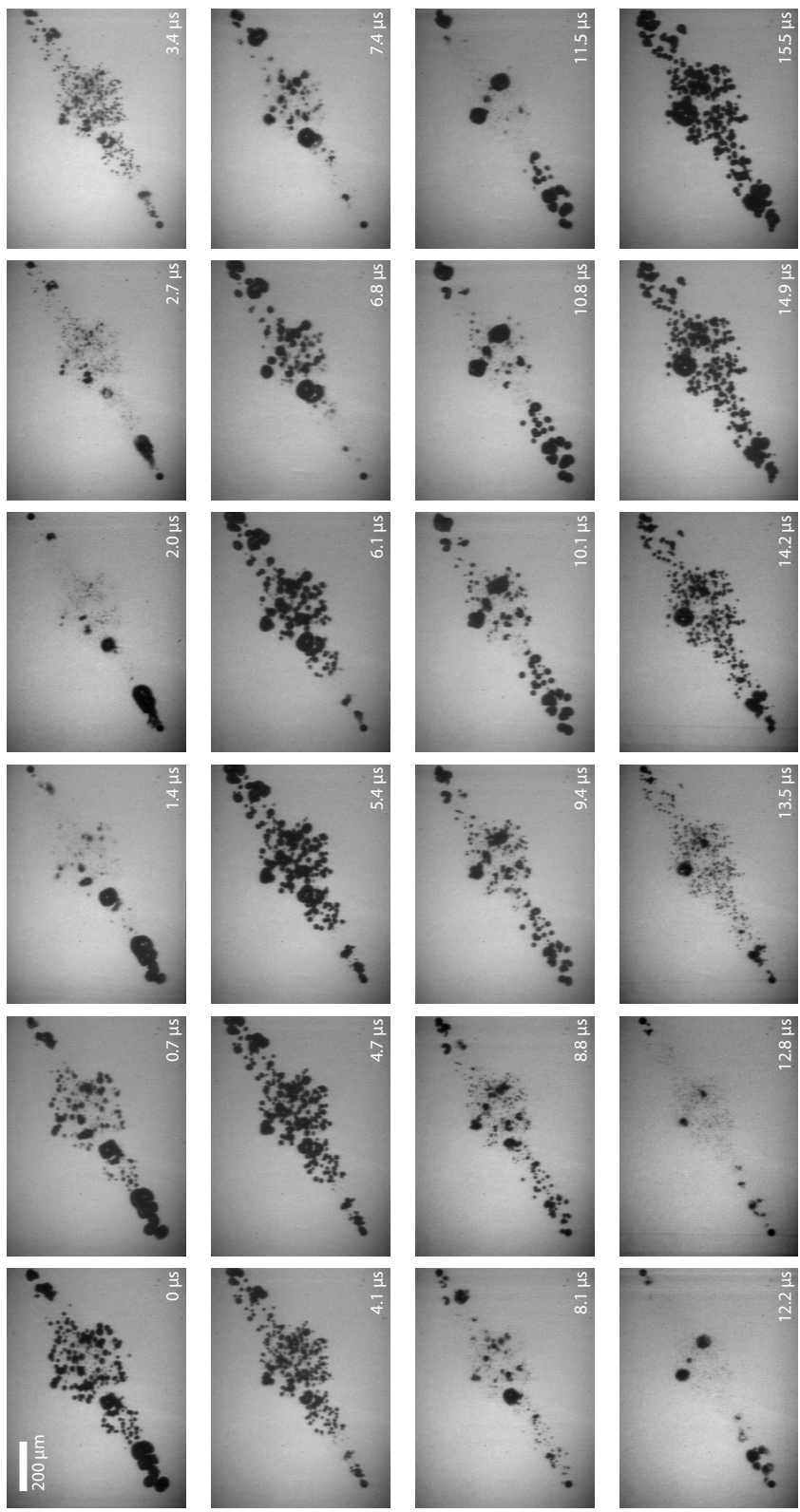

Figure 5.12: Frames from a high-speed recording of the dual micropit cavitation cloud at $810 \mathrm{~mW}$ input power and imaged at $1.4 \mathrm{Mfps}$.

SEM Zeiss 1550) shown in Figure 5.13 reveal that small segments with dimensions typically less than $1 \mu \mathrm{m}$ are chipped out of the surface. Note that the shapes of the damaged sections seem to coincide with the crystal orientation planes $\{111\}$ of the 
crystalline structure of the silicon (100) substrate.
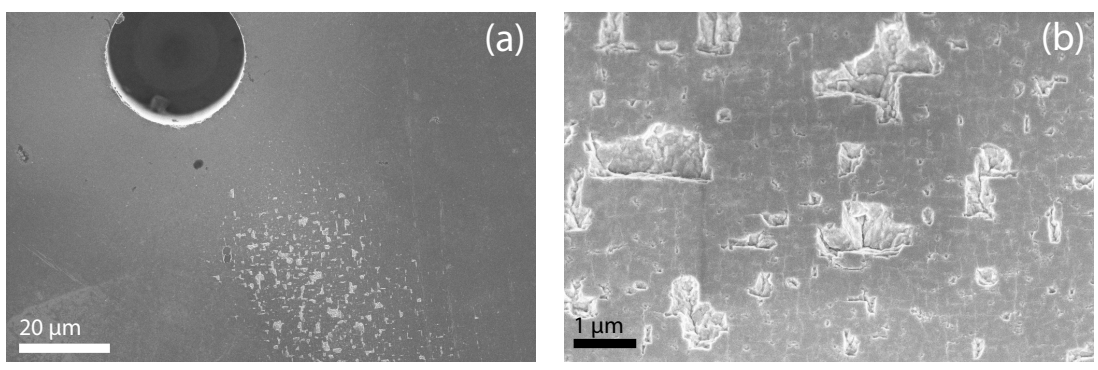

Figure 5.13: (a) Cavitation-induced surface damage next to the micropit and detailed in (b).

Another demonstration of the forces induced by the collapse of cavitation bubbles is the occurrence of shock waves. Here, the ultrashort flash photography method enabled shadowgraphy imaging of these waves (Figure 5.14). The shock wave shown in the image originates from the collapse of the bubble cluster indicated by the arrow. Assuming the wave to move at a velocity equal to the speed of sound in water (approximately $1500 \mathrm{~m} / \mathrm{s}$ ) the collapse occurred some $0.22 \mu$ s before this image was taken. Violent bubble collapse is also associated with the generation of surface damage, therefore it is possible that shock wave emission is an indication of such an event. Images similar to Figures 5.14 and 6.7 show circular shock wave emissions originating from many different locations within the cavitation cloud. Further details on the damage of different crystallographic silicon substrates are given elsewhere [39] and Chapter 8 .

\subsection{Discussion \& Conclusions}

The introduction of artificially created crevice bubbles into an acoustically insonified liquid can result in a myriad of acoustic cavitation related phenomena. Streams of microbubbles are released into the liquid and cavitation bubble clouds are formed at elevated acoustic pressures. The remarkable stability of these processes is enabled by the diffusion of gas back into the micropit bubble. The results of the exploratory study described here confirms the common assumption that cavitation activity can be initiated and enhanced by the presence of crevice bubbles. However, the mechanism with which bubble release transpires is different from bubble pinch-off occurring in e.g. nucleate boiling and supersaturated liquids. In these heat and gas diffusion driven processes, the bubble displays unstable growth outside the perimeter of the pit after which it detaches due to buoyancy or drag forces. The inception of a cavitation bubble due to pinch-off from a micropit bubble does not appear to involve such nucleation and subsequent detachment. Here, the released bubbles are typically smaller than the 


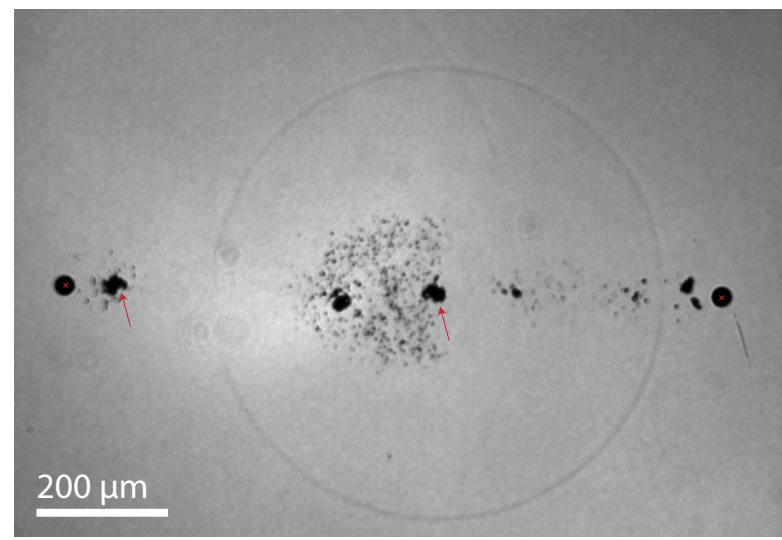

Figure 5.14: A shock wave originating from a cavitation bubble collapse close to the center of the dual-micropit bubble cloud (indicated with the red arrow in the center). The two micropits are at both extreme sides. Parts of another shock wave from an earlier event can also be distinguished, the origin of which is indicated with the left arrow.

micropit bubble and may result from large amplitude capillary waves which locally "fold" the liquid-gas interface into a singularities.

In this study three different frequencies were applied, each displaying distinct types of bubble generation and cavitation activity. Evidently for improved understanding a much larger parameter space should be explored. In particular the effect of driving frequency, amplitude, pit dimensions and numbers are of interest.

Clearly, the use of artificial crevice bubbles constitutes a new method (with advantages over conventional US horns or baths) to initiate and enhance cavitation at any desired location, provided the liquid and acoustic parameters are appropriate. The unique control over cavitation activity enables detailed investigation of acoustic cavitation and its effects such as shock wave emission, damage formation or sonochemistry, as was demonstrated in previous studies [7,-9, 39] (Chapters 34, 6, 7] and 8). Future studies on pit-bubble oscillation and pinch-off events are still needed to understand more about this phenomenon (see Chapter 9). The introduction of micropits into a system may also proof beneficial for many applications based on acoustic cavitation.

\section{Acknowledgements}

This study was funded by IMEC vzw. The authors would like to acknowledge the support in fabrication techniques provided by Stefan Schlautmann. The experiments in glass substrates were conducted at Nangyang University, Singapore with the support of Prof. Claus Dieter Ohl and Dr. Tandiono. 


\section{References}

[1] C. Brennen, Cavitation and Bubble Dynamics, Oxford engineering science series (Oxford University Press) (1995).

[2] K. Suslick, Y. Didenko, M. Fang, T. Hyeon, K. Kolbeck, W. McNamara, M. Mdleleni, and M. Wong, "Acoustic cavitation and its chemical consequences", Philos. Trans. R. Soc. London, Ser. A 357, 335 (1999).

[3] M. Ashokkumar, "The characterization of acoustic cavitation bubbles: An overview", Ultrason. Sonochem. 18, $864-872(2011)$.

[4] K. Yasuda, T. Torii, K. Yasui, Y. Iida, T. Tuziuti, M. Nakamura, and Y. Asakura, "Enhancement of sonochemical reaction of terephthalate ion by superposition of ultrasonic fields of various frequencies", Ultrason. Sonochem. 14, 699-704 (2007).

[5] S. Muthukumaran, K. Yang, A. Seuren, S. Kentish, M. Ashokkumar, G. W. Stevens, and F. Grieser, "The use of ultrasonic cleaning for ultrafiltration membranes in the dairy industry", Sep. Purif. Technol. 39, 99 - 107 (2004).

[6] T. J. Mason, L. Paniwnyk, and J. P. Lorimer, "The uses of ultrasound in food technology", Ultrason. Sonochem. 3, S253 - S260 (1996).

[7] D. Fernandez Rivas, A. Prosperetti, A. G. Zijlstra, D. Lohse, and J. G. E. Gardeniers, "Efficient sonochemistry through microbubbles generated with micromachined surfaces", Angew. Chem. Int. Ed. 49, 9699-9701 (2010).

[8] D. Fernandez Rivas, L. Stricker, A. G. Zijlstra, J. G. E. Gardeniers, D. Lohse, and A. Prosperetti, "Ultrasound artificially nucleated microbubbles and their sonochemical radical production", Ultrason. Sonochem. Accepted, - (2012).

[9] D. Fernandez Rivas, M. Ashokkumar, T. Leong, K. Yasui, T. Tuziuti, S. Kentish, D. Lohse, and J. G. E. Gardeniers, "Sonoluminescence and sonochemiluminescence from a microreactor", Ultrason. Sonochem. 19, 1252-1259 (2012).

[10] R. E. Apfel, “Acoustic cavitation inception”, Ultrasonics 22, 167 - 173 (1984).

[11] S. Helbig, S. Urban, E. Klein, and S. Singh, "Impact of megasonic process conditions on pre and sub-resolution assist feature damage", Photomask Technology 7122, 712210 (2008).

[12] W. J. Greenleaf, M. E. Bolander, G. Sarkar, M. B. Goldring, and J. F. Greenleaf, "Artificial cavitation nuclei significantly enhance acoustically induced cell transfection”, Ultrasound Med. Biol. 24, 587 - 595 (1998).

[13] D. M. Hallow, A. D. Mahajan, T. E. McCutchen, and M. R. Prausnitz, "Measurement and correlation of acoustic cavitation with cellular bioeffects", Ultrasound Med. Biol. 32, 1111 - 1122 (2006).

[14] K. M and M. Goto, "Size control of nanobubbles generated from shirasu-porous-glass (spg) membranes", J. Membr. Sci. 281, 386 - 396 (2006).

[15] B. Dollet, W. van Hoeve, J. P. Raven, P. Marmottant, and M. Versluis, "Role of the channel geometry on the bubble pinch-off in flow-focusing devices", Phys. Rev. Lett. 100, 034504 (2008).

[16] E. N. Harvey, D. K. Barnes, W. D. McElroy, A. H. Whiteley, D. C. Pease, and K. W. Cooper, "Bubble formation in animals. i. physical factors", J Cell Comp Physiol. 24, 1-22 (1944).

[17] A. A. Atchley and A. Prosperetti, "The crevice model of bubble nucleation", J. Acoust. Soc. Am. 86, 10651084 (1989)

[18] S. F. Jones, G. M. Evans, and K. P. Galvin, "Bubble nucleation from gas cavities - a review”, J. Coll. Interf. Sci. 80, 27 - 50 (1999). 
[19] G. Liger-Belair, M. Vignes-Adler, C. Voisin, B. Robillard, and P. Jeandet, "Kinetics of gas discharging in a glass of champagne: The role of nucleation sites", Langmuir 18, 1294-1301 (2002).

[20] C. Y. Han and P. Griffith, "The mechanism of heat transfer in nucleate pool boiling", Technical Report, Massachusetts Institute of Technology. Heat Transfer Laboratory (1962).

[21] N. Bremond, M. Arora, C. D. Ohl, and D. Lohse, "Cavitation on surfaces", Journal of Physics: Condensed Matter 17, S3603 (2005).

[22] N. Bremond, M. Arora, C. D. Ohl, and D. Lohse, "Controlled multibubble surface cavitation", Phys. Rev. Lett. 96, 224501 (2006).

[23] B. M. Borkent, S. Gekle, A. Prosperetti, and D. Lohse, "Nucleation threshold and deactivation mechanisms of nanoscopic cavitation nuclei”, Physics of Fluids 21, 102003 (2009).

[24] S. Kentish, J. Lee, M. Davidson, and M. Ashokkumar, "The dissolution of a stationary spherical bubble beneath a flat plate", Chemical Engineering Science 61, 7697 - 7705 (2006).

[25] C. H. Wang and V. K. Dhir, "Effect of surface wettability on active nucleation site density during pool boiling of water on a vertical surface", Journal of heat transfer 115, 659-669 (1993).

[26] D. E. Hughes and W. L. Nyborg, “Cell disruption by ultrasound”, Science 138, pp. 108-114 (1962).

[27] S.-D. Howkins, "Solid erosion in low-amplitude sound fields", The Journal of the Acoustical Society of America 39, 55 (1966).

[28] Tandiono, S. Ohl, D. Ow, E. Klaseboer, V. Wong, R. Dumke, and C. Ohl, "Sonochemistry and sonoluminescence in microfluidics", Proc. Natl. Acad. Sci. USA 108, 5996-5998 (2011).

[29] C. T. Chin, C. Lancée, J. Borsboom, F. Mastik, M. E. Frijlink, N. de Jong, M. Versluis, and D. Lohse, "Brandaris 128: A digital 25 million frames per second camera with 128 highly sensitive frames", Rev. Sci. Instr. 74, 5026-5034 (2003)

[30] F. P. Schäfer, W. Schmidt, and J. Volze, "Organic dye solution laser”, Applied Physics Letters 9, 306-309 (1966).

[31] A. van der Bos, A. Zijlstra, E. Gelderblom, and M. Versluis, "ilif: illumination by laser-induced fluorescence for single flash imaging on a nanoseconds timescale”, Exp. Fluids 51, 1283-1289 (2011).

[32] G. D. Crapper, "An exact solution for progressive capillary waves of arbitrary amplitude”, J. Fluid Mech. 2, 532-540 (1957).

[33] C. E. Brennen, Cavitation and bubble dynamics (Oxford University Press, New York) (1995).

[34] H. Gelderblom, A. Zijlstra, L. van Wijngaarden, and A. Prosperetti, "Oscillations of a gas pocket on a liquidcovered solid surface", Phys. Fluids (2012).

[35] A. Eller and H. G. Flynn, "Rectified diffusion during nonlinear pulsations of cavitation bubbles", J. Acoust. Soc. Am. 37, 493-503 (1965).

[36] D. L. Miller, "Particle gathering and microstreaming near ultrasonically activated gas-filled micropores", J. Acoust. Soc. Am. 84, 1378-1387 (1988).

[37] D. Krefting, R. Mettin, and W. Lauterborn, "High-speed observation of acoustic cavitation erosion in multibubble systems", Ultrason. Sonochem. 11, 119 - 123 (2004).

[38] M. Dular and A. Osterman, "Pit clustering in cavitation erosion”, Wear 265, 811 - 820 (2008).

[39] D. Fernandez Rivas, J. Betjes, B. Verhaagen, W. Bouwhuis, T.-C. Bor, D. Lohse, and J. Gardeniers, "Erosion evolution in mono-crystalline silicon surfaces caused by acoustic cavitation bubbles", J. Appl. Phys. to be submitted (2012). 


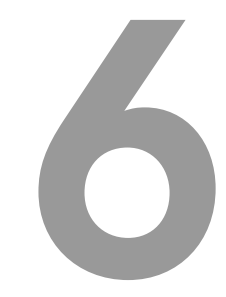

\title{
Ultrasound Artificially Nucleated Bubbles and their Sonochemical Radical Production *
}

\begin{abstract}
We describe the ejection of bubbles from air-filled pits micromachined on a silicon surface when exposed to ultrasound at a frequency of approximately $200 \mathrm{kHz}$. As the pressure amplitude is increased the bubbles ejected from the micropits tend to be larger and they interact in complex ways. With more than one pit, there is a threshold pressure beyond which the bubbles follow a trajectory parallel to the substrate surface and converge at the center point of the pit array. We have determined the size distribution of bubbles ejected from one, two and three pits, for three different pressure amplitudes and correlated them with sonochemical $\mathrm{OH}$ radical production. Experimental evidence of shock wave emission from the bubble clusters, deformed bubble shapes and jetting events that might lead to surface erosion are presented. We describe numerical simulations of sonochemical conversion using the empirical bubble size distributions, and compare the calculated values with experimental results.
\end{abstract}

${ }^{*}$ David Fernandez Rivas, Laura Stricker, Aaldert G. Zijlstra, Han J. G. E. Gardeniers, Detlef Lohse and Andrea Prosperetti, Ultrasound artificially nucleated bubbles and their sonochemical radical production, in press in Ultrasonic Sonochemistry, 2012. doi:10.1016/j.ultsonch.2012.07.024 


\subsection{Introduction}

Sonochemistry, the use of ultrasound in chemistry and processing [1], is a very promising field with applications in e.g. nanomaterials synthesis, degradation of contaminants in water treatment and the food industry [2-4]. In a sonochemical reactor each bubble acts as a single reactor in itself [5, 6], but the spatial distribution of these bubbles is normally not homogeneous, forming filamentary patterns or clusters [7, 8]. Establishing the correlation between the sonochemical yield and the size, location, and dynamics of the bubbles in these clusters, may provide the knowledge to improve the efficiency of sonochemical reactors.

There is a considerable amount of experimental and theoretical work on single bubbles [5, 9, 10] but, for practical applications, where the interest normally resides in multibubble systems, it turns out to be very difficult to extract bubble sizes and spatial distributions [11, 12]. So far only holographic or laser techniques were successful in providing such information while also valuable information was obtained from bubble dissolution measurement [7, 13-19]. No study combining bubble distribution, sonochemical conversion and corresponding numerical simulations has been published.

At the root of this problem lies the fact that cavitation and its inception are extremely complex and very difficult to control. Once the conditions for cavitation inception exist, many phenomena have an influence on the functioning of the sonochemical reactor: bubble-bubble interaction, coalescence, surfactants or impurities dissolved in the liquid altering the bubble population and liquid properties, recirculation of the liquid inside the reactor, energy losses due to viscous heating, degassing of the liquid, energy conversion losses (e.g., electrical-to-mechanical) among others [20-28]. In this work we present a continuation of our first efforts to increase the efficiency of sonochemical reactors [29] (Chapter 6). The concept is based on small predefined crevices in which stabilized gas pockets remain entrapped when introduced into the liquid [30-33]. When exposed to ultrasound, these gas pockets continuously generate chemically active cavitation bubbles at their location. These bubbles form peculiar and stable clouds in the reactor that do not occur in the absence of the pits.

A major advantage of this method is that the location of the bubbles is stable, known a priori, and coincides with the region of high intensity ultrasound. This feature is in contrast with the usual sonoreactors where bubbles occur randomly over large volumes. The reproducible cavitation structures generated in our system enable us to study the relation between the bubble size distribution, number of bubbles, spatial distributions, and chemical production rates. The latter are determined using dosimetry of $\mathrm{OH}$ radicals while the former are obtained using a nanosecond flashphotography technique. In this paper, the acquired rates in terms of radical production per bubble per acoustic cycle are discussed. Additionally, calculations provide further 
insight into some of the observed phenomena.

\subsection{Materials and Methods}

\subsubsection{Silicon micromachining}

The bubbles were generated from the gas entrapped in pits with a diameter of $30 \mu \mathrm{m}$ and a depth of $10 \mu \mathrm{m}$ etched in square silicon chips with a side of $10 \mathrm{~mm}$. These substrates were micromachined under clean room conditions on double-side polished silicon wafers with (100) crystallographic orientation. The pits were etched by means of a plasma dry-etching machine (Adixen AMS 100 SE, Alcatel). Pits arranged in three different configurations were used: single pits, two pits separated by a distance of $1 \mathrm{~mm}$, and three pits arranged at the corners of an equilateral triangle with sides of $1 \mathrm{~mm}$ (see Fig. 6.1).

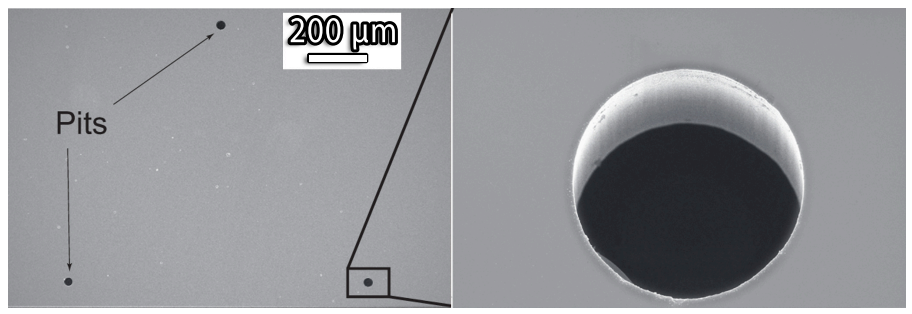

Figure 6.1: Pits micromachined on a silicon substrate by deep reactive ion etching. Top view (left) and a zoom-in perspective view (right). The diameter of the pit on the right is $30 \mu \mathrm{m}$.

\subsubsection{Set-up for US experiments and imaging technique}

A sketch of the experimental arrangement is shown in Fig. 6.2 .

The cavitation cell was a glass container of $25 \mathrm{~mm}$ outer diameter, $15 \mathrm{~mm}$ inner diameter and $6 \mathrm{~mm}$ depth. The thickness of the cell bottom was $6 \mathrm{~mm}$ and matched one quarter of the wavelength at the operation frequency of $200 \pm 5 \mathrm{kHz}$ generated by a piezo Ferroperm PZ27 $6 \mathrm{~mm}$ thick and $25 \mathrm{~mm}$ in diameter glued to the cell bottom. To prevent loss of water by evaporation and gas by acoustic degassing the cell was covered by a glass slide resting on a rubber ring.

Two types of experiments were conducted, the measurement of sonochemical reaction rates and the imaging of the bubbles and their size distribution. For the first type, the acoustic excitation was a sinusoidal signal provided by a Hewlett Packard 33120A arbitrary waveform generator amplified by means of a SONY TA-FB740R QS amplifier. The voltage and current provided to the piezo were measured with a Tektronix DPO 4034 oscilloscope and Tektronix current probe CTA2. The bottom 


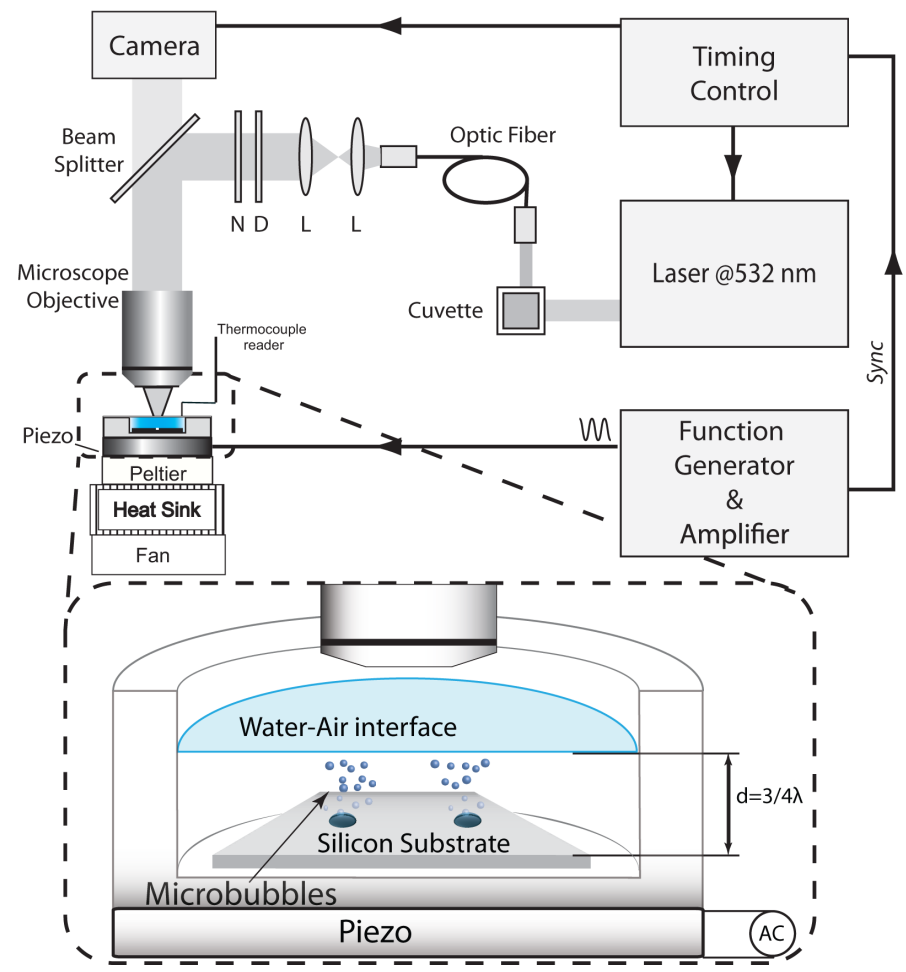

Figure 6.2: Experimental setup used for short exposure imaging and inset showing a detailed sketch of the cavitation cell.

surface of the piezo was kept in contact with a Peltier element (Marlow Industrial) to maintain the temperature at a constant value of $25^{\circ} \mathrm{C}$. At low and medium power the temperature increase during each experiment was measured to be no more than $1 \mathrm{~K}$, while in the high-power case the temperature increased no more than $3.8 \mathrm{~K}$, mainly during the first minute, and then remained constant. To study the radical production three different power settings were used with all three pit arrangements: low $(0.074$ $\mathrm{W})$, medium $(0.182 \mathrm{~W})$ and high $(0.629 \mathrm{~W})$.

The arrangement was similar for the bubble imaging experiments except for the use of a Krohn-Hite model 7602 wide-band amplifier and a Tabor Electronics Ltd. model WW1072 function generator. The different amplifiers resulted in differences in the electrical power supplied to the piezo element, which were $0.194 \mathrm{~W}$ (low), 0.654 (medium) and $0.981 \mathrm{~W}$ (high). In this case space constraints prevented the use of the Peltier element.

Imaging was carried out with two cameras, one Photron model SA1.1 recording movies at normal speed ( $25 \mathrm{fps})$, the other one used for single snapshots was a Lumenera LM165 with a sensitive Sony EXview HAD CCD sensor. The Olym- 
pus microscope was equipped with two long working distance (WD) objectives, the LMPLFLN 10×, (WD $21 \mathrm{~mm} / \mathrm{NA}=0.25)$ and the SMPLFLN $20 \times(\mathrm{WD} 25 \mathrm{~mm} / \mathrm{NA}=$ 0.25 ) both Olympus. Illumination was provided by a bright laser induced fluorescence pulse of $7 \mathrm{~ns}$ duration (full width at half maximum) which, amounting to about one thousandth of the acoustic period, enabled us to obtain high-resolution images without motion blur. The minimum radius detectable in the experiment was $\sim 0.5$ $\mu \mathrm{m}$. Additional details on this method can be found elsewhere [34]. Examples of the images obtained in this way are shown in Figures 6.3 and 6.4

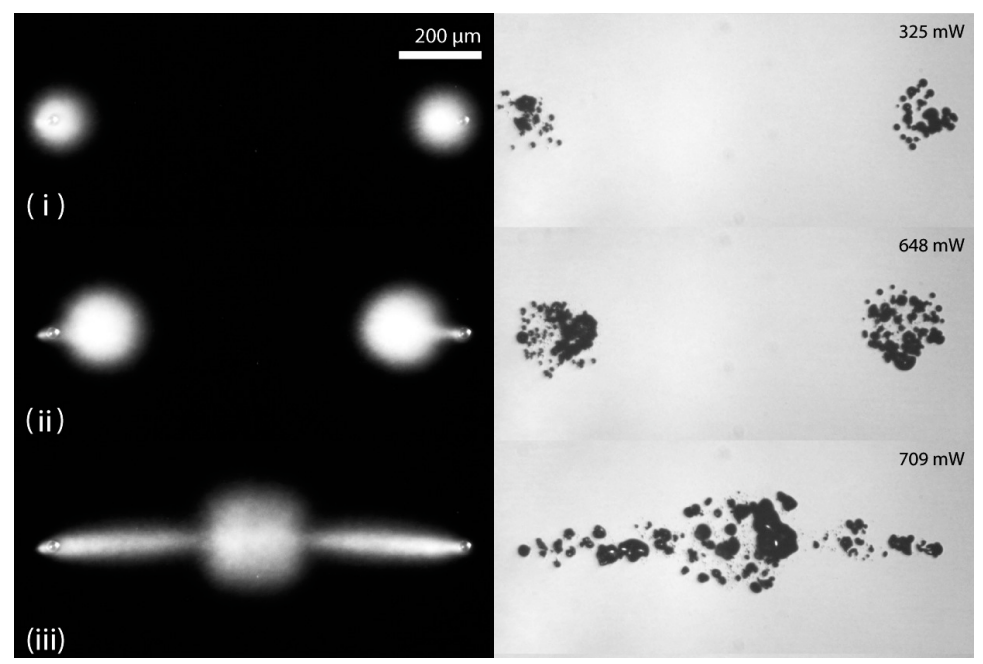

Figure 6.3: Bubble clouds originating from a two-pits substrate. Each row corresponds to a different acoustic amplitude increasing from top to bottom. The left column illustrates the time averaged extent of the cavitation cloud imaged at normal speed. The right column are single snapshots of 7 ns exposure time, in which individual bubbles can be distinguished at approximately maximum expansion.

The purpose of these images was to obtain information on the number and sizes of the bubbles at various phases of the sound excitation. The difficulty to overcome was that the bubble density was high so that in many cases the images of different bubbles overlapped. To identify the individual bubbles it was therefore necessary to develop a special Matlab-based software using the so-called watershed segmentation algorithm [35]. After identification, the bubbles were counted and the image of each bubble was processed to extract the projected area and, with the assumption of a spherical shape, the equivalent diameter.

\subsubsection{Pressure amplitudes}

The quantity of interest for bubble oscillations is the acoustic pressure amplitude rather than the electrical power provided to the transducer. Unfortunately the conver- 
sion from one to the other is a notoriously difficult step. We attempted to measure the acoustic pressure amplitude by using a hydrophone (Onda HNR-500) with the tip positioned $65 \mu \mathrm{m}$ above the center point of the chip. The estimated error affecting these values according to the manufacturer is $20 \%$. The values found in this way were $165 \mathrm{kPa}$ for $0.074 \mathrm{~W}, 225 \mathrm{kPa}$ for $0.182 \mathrm{~W}$, and $350 \mathrm{kPa}$ for $0.629 \mathrm{~W}$. These values are uncertain due to several possible factors, electrical interference, the presence of waves on the surface of the liquid, bubble formation at the hydrophone tip and others. Furthermore, bubbles detune the cell and shield each other, therefore these values are provided only for reference purposes. A better estimate will be derived in Section 6.3 .5 with the aid of numerical simulations.

\subsubsection{Radical dosimetry}

Conversion of terephthalic acid to 2-hydroxyterephthalic acid (HTA) was taken as a measure for the concentration of $\mathrm{OH}$ radicals. A calibration graph of fluorescence intensity as a function of HTA concentration was obtained following steps similar to those described in the literature [36]. Fluorescence was measured using a spectrofluorometer (TECAN Sapphire ${ }^{2 T M}$ ) with an excitation wavelength of 310 $\mathrm{nm}$. The fluorophore had a peak emission wavelength of $429 \mathrm{~nm}$, but the emission was scanned between 350 and $600 \mathrm{~nm}$. Following the method reported by Iida [37], the acid solution was prepared by mixing $2.0 \times 10^{-3} \mathrm{~mol} / \ell(0.332 \mathrm{~g})$ of terephthalic acid (Sigma-Aldrich) $5.0 \times 10^{-3} \mathrm{~mol} / \ell(0.200 \mathrm{~g})$ of $\mathrm{NaOH}$ and phosphate buffer (pH 7.4), prepared from $4.4 \times 10^{-3} \mathrm{~mol} / \ell(0.589 \mathrm{~g})$ of $\mathrm{KH}_{2} \mathrm{PO}_{4}$ and $\mathrm{Na}_{2} \mathrm{HPO}_{4}(7.0$ $\times 10^{-3} \mathrm{~mol} / \ell(0.981 \mathrm{~g})$ ) (all from Riedel - de Haën). The resulting solution was then made up to $1 \ell$ with milliQ water.

An amount of $300 \mu \ell$ of the solution measured with an Eppendorf micropipette was used in each experiment. At the end of the experimental run, the solution was pipetted out of the cell and stored in dark in sterilized vials (manufactured by Brand) for spectroscopic analysis. This step was conducted with $200 \mu \ell$ taken from the stored solution pipetted into the wells of a Corning Inc. well assay plate. The spectrophotometer settings for the analysis of the samples were: gain 40, height from the well, $9000 \mu \mathrm{m}$, integration time $100 \mu \mathrm{s}, 10$ reads per well.

Each experiment was repeated six to ten times and the mean concentration value was calculated. The largest standard deviation occurring for the case of three pits at maximum power, was about $30 \%$ of the average value.

Calculation of the radical formation rate was carried out according to:

$$
\frac{\Delta N_{\text {rad }}}{\Delta t}=\frac{\left(c_{\text {end }}-c_{\text {initial }}\right) N_{A} V}{\Delta t},
$$

in which $c_{\text {end }}$ and $c_{\text {initial }}=0$ are the final and initial concentrations of HTA in moles per volume, $N_{A}=6.02 \times 10^{23} \mathrm{~mol}^{-1}$ is Avogadro's number and $V$ is the liquid volume 
exposed to the US $(300 \mu \ell)$.

\subsubsection{Theoretical bubble dynamics and radical production calculation}

The model used to theoretically describe the bubble dynamics is based on an extended Rayleigh-Plesset-type equation, adopting the boundary layer approximation to estimate diffusive and thermal fluxes and assuming spherical symmetry for the bubble [38-41]. Temperature evolution over time is given by a global energy balance. The gas inside the bubble is taken as air with a van der Waals type equation of state. Changes in the transport parameters in accordance with compositional changes of the mixture are also included in the model. A driving frequency of $200 \mathrm{kHz}$ was taken, in agreement with experiments. Interaction between bubbles due to Bjerknes forces as well as coalescence effects were neglected.

\subsection{Results and Discussion}

\subsubsection{Description of the observed phenomena}

When the US is switched on, a continuous generation of bubbles is observed issuing from the gas stabilized in the pits on the silicon surface. These bubbles are likely caused by an instability of the liquid free surface at the pit mouth which undergoes large-amplitude oscillations under the action of the US. A similar process taking place at the surface of a free bubble has been described in the literature [7, 42, 43] and Chapter 5 .

The resonance frequency of the pits is of the order of $150 \mathrm{kHz}$ and the radius of bubbles resonating at the applied frequency of $200 \mathrm{kHz}$ is about $15 \mu \mathrm{m}$ [44, 45]. Thus one would expect that, under the action of Bjerknes forces, bubbles smaller than this size would be repelled by the pits while larger ones would be attracted. At low power, the observed behavior is compatible with this expectation as shown in Figures 6.3 and 6.4. A few bubbles are observed at any given time. They move some distance away from the pit and survive a few cycles before disappearing [44].

As the power is increased, the ejected bubbles become more numerous forming clouds of increasingly larger size around the pits. At still higher powers, with more than one pit, the clouds appear to be attracted to each other until, at a fairly reproducible power level, they travel towards the central area of the pit array where they collect forming a single cloud (see Figures 6.3 and 6.4). As shown in an earlier paper [29] (Chapter 3), and as can be seen in the last frame of Fig. 6.8 below, these clouds when observed from the side resemble the conical clouds formed at the tip of sonotrodes [46, 47]. These structures are very different from the filamentary structures, sometimes called acoustic Lichtenberg figures [48], which are common in normal acoustic cavitation at high acoustic amplitudes. 


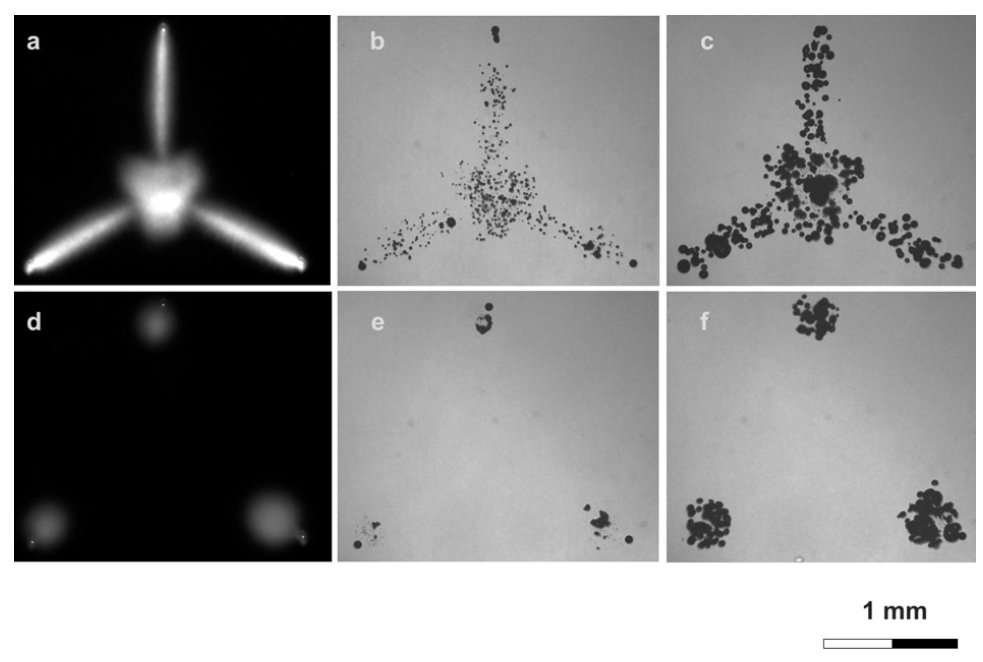

Figure 6.4: Images of a substrate with three pits at different powers and at two selected points in the acoustic cycle. The upper row corresponds to high power $(0.981 \mathrm{~W})$ and the lower row to low power $(0.194 \mathrm{~W})$. Visible with normal illumination conditions $(a$ and $d)$ and corresponding short exposure images: $b$ and $e$ in the compression; $c$ and $f$ in the expansion phase of the acoustic cycle.

The cloud is continually fed by new bubbles arriving from the pits under the action of the complex interplay of primary and secondary Bjerknes forces and microstreaming acting on the bubbles. The bubbles in the cloud are observed to break up seeding the liquid with their fragments which then grow into additional bubbles that join the cloud. The processes responsible for the accretion and loss of bubbles eventually reach a steady state and the cloud is not observed to grow further.

The transition from clouds localized near the pits to a central cloud exhibits hysteresis, with the pressure amplitude necessary to return to separated clouds lower than that needed to bring them together.

The three-pits arrangement of Fig. 6.4 shows transition to a pattern similar to that of Fig. 6.3 for two pits when one of the three pits is rendered inactive (see Fig. 6.5). This observation suggests that liquid flow does not play a major role in the observed cloud attraction, which therefore must be mostly due to Bjerknes forces.

At high power a region of damaged silicon substrate forms near the pits within 5 minutes of exposure to the US (see Fig. 6.6). The shape of the individual damage sites coincides with the crystal planes of the crystalline structure of the silicon wafers. Damage to other parts of the chip and, in particular, under the large cloud forming over the center of the pit array, is much more modest and takes a significantly longer time. For more details see Chapter 8

We have found that this localized acoustic bubble generation can be sustained 

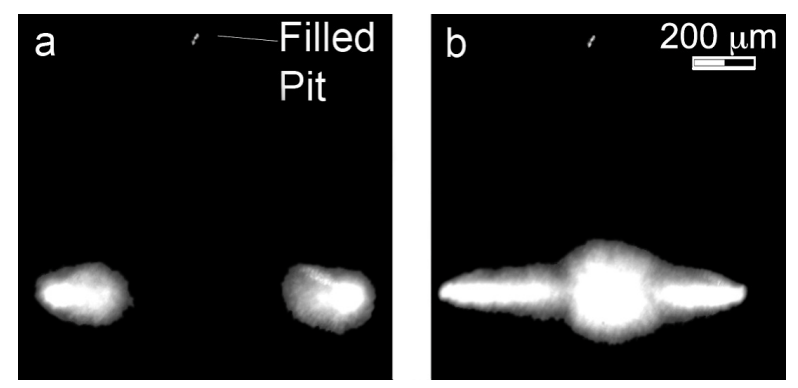

Figure 6.5: When a pit is filled with water in a three-pit substrate, the bubbles nucleated by the remaining active pits attain the same configuration as for a two-pit substrate: a, Medium power $(0.654 \mathrm{~W})$ and $b$, High power (0.981).

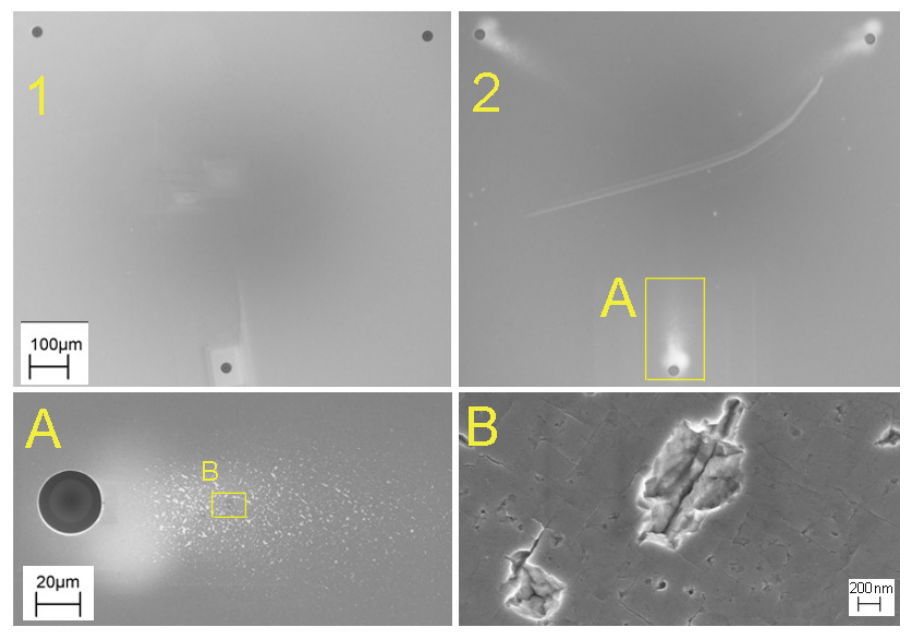

Figure 6.6: SEM image affording a comparison of a substrate before (1) and after (2) use in ultrasonic experiments. The damage pattern and zoom-in views of it, show that the eroded surface coincides with the $\{111\}$ silicon crystal planes.

for at least several hours if temperature is kept constant and gas loss is prevented by means of the glass slide mentioned before. Therefore we must conclude that the loss of gas associated to the bubble generation does not deactivate the pits, which is evidence of a process of rectified gas diffusion into the pits similar to the well-known process taking place with free bubbles.

The emission of shocks by collapsing bubbles is a known feature [49, 50]. An example in our case is shown in Fig. 6.7.

When bubbles are close to each other or to the solid surface, which preferentially occurs at higher power, they tend to deform and to interact. When bubbles are close enough, they develop liquid jets towards each other (see as an example [51]). Fig. 

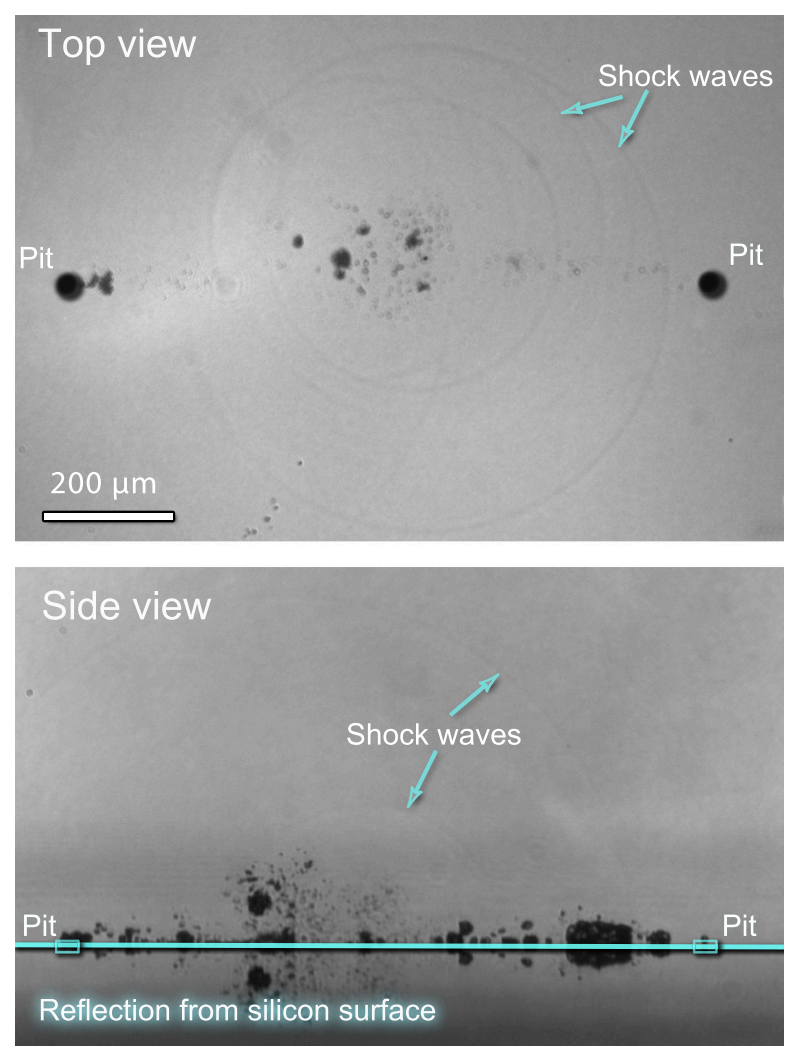

Figure 6.7: Shock waves emitted in the two-pit, high power situation $(0.981 \mathrm{~W})$. Several shock waves are observed in top and side views.

6.8 shows several examples of such deformations and interactions.

In interpreting these images it must be kept in mind that bubbles smaller than resonance size expand during the acoustic compression phase, while bubbles bigger than resonance size contract. At high powers these latter bubbles may become unstable and split, generating the significantly smaller bubbles seen in the figure. The actual stability of these bubbles will also be influenced by their distance from the solid substrate and the proximity of other bubbles. Some of the smaller bubbles may also become unstable and fragment during collapse, but this would happen during the acoustic expansion phase.

We have estimated the translational velocity of typical bubbles on the basis of some preliminary experiments by imaging the acoustic field at $1.4 \mathrm{Mfps}$. We found typical velocities of about $10 \mathrm{~m} / \mathrm{s}$ and corresponding lifetimes of $100 \mu \mathrm{s}$, which is of the same order as that reported for other typical sonoreactors at similar frequencies [18] where bubbles had a lifetime of the order of $350 \mu \mathrm{s}$. 


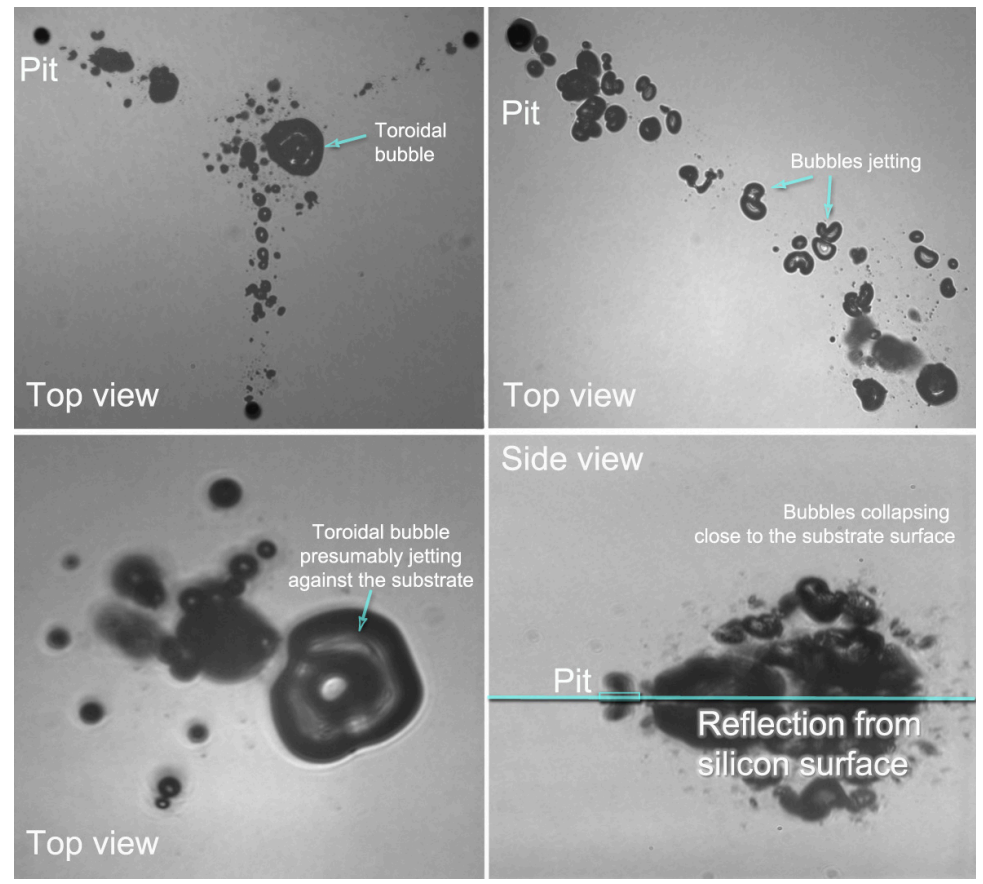

Figure 6.8: Deformed bubbles at high power settings $(0.981 \mathrm{~W})$. Note how bubbles tend to deform in all cases. When two bubbles are close, they develop liquid jets towards each other as in the upper right picture.

\subsubsection{Bubble size distribution}

As explained before, in order to characterize the bubble size distribution and its evolution in time over an acoustic period, we took snapshots of the cavitating region with an exposure of $7 \mathrm{~ns}$. The repetition time of the laser did not allow us to take consecutive images faster than about 1 per second, i.e., every 200,000 acoustic cycles. The laser timing was set so as to generate a flash at a fixed phase of the sound field. We selected 10 phases and took 10 images per phase to improve the statistics.

As shown in Table 6.1, at the low power settings the number of bubbles in each frame was relatively small, typically 3-5 per pit and sometimes even less. Furthermore, the occasional overlaps of the bubble and pit images made it difficult to accurately count these bubbles. The measured low-power bubble populations, therefore, are not very accurate but are presented for reference as a lower bound; an example is provided in Fig. 6.9 .

At the medium power settings the statistics improves since the bubbles tend to be more numerous, to grow larger and to move away from the pits. An example of a probability density function (PDF) for medium power is shown in Fig. 6.10 .

At high power $(0.981 \mathrm{~W})$ and one-pit configuration the expansion and collapse 


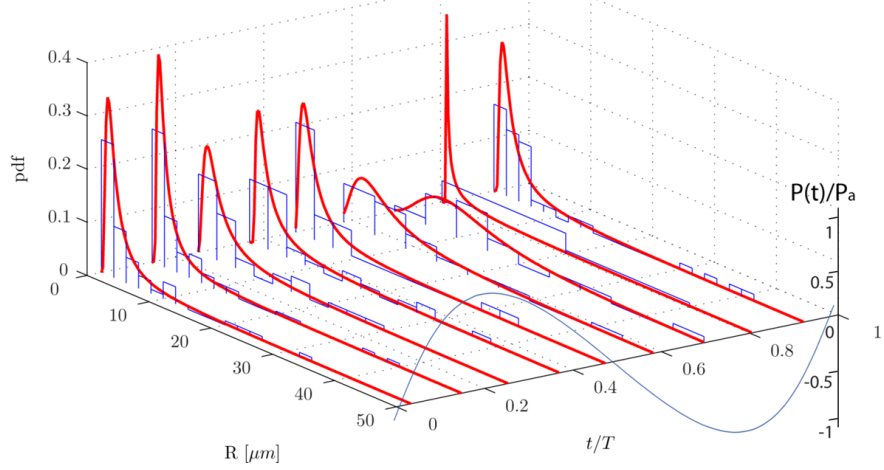

Figure 6.9: Bubble size distribution histograms at a power of $0.194 \mathrm{~W}$ for one pit. The axis to the extreme right represents the normalized pressure for the acoustic cycle.

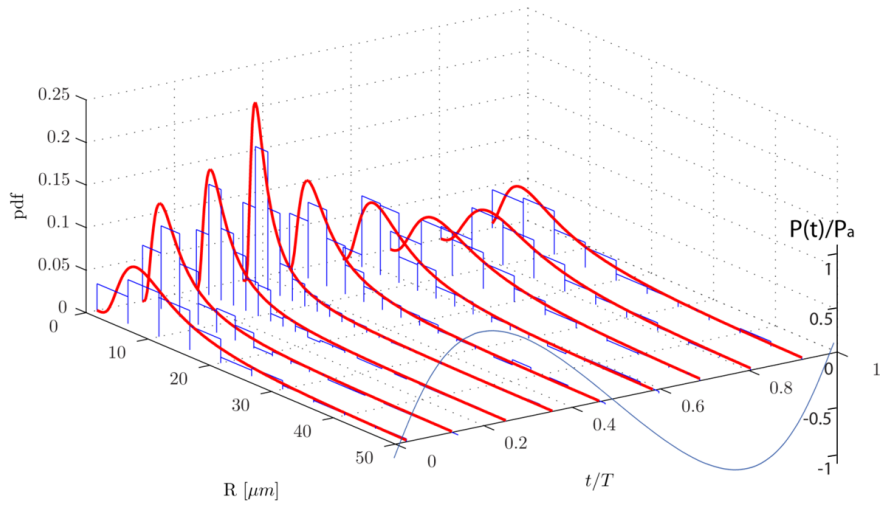

Figure 6.10: Bubble size distribution histograms for a power of $0.654 \mathrm{~W}$ for two pits. The axis to the extreme right represents the normalized pressure for the acoustic cycle.

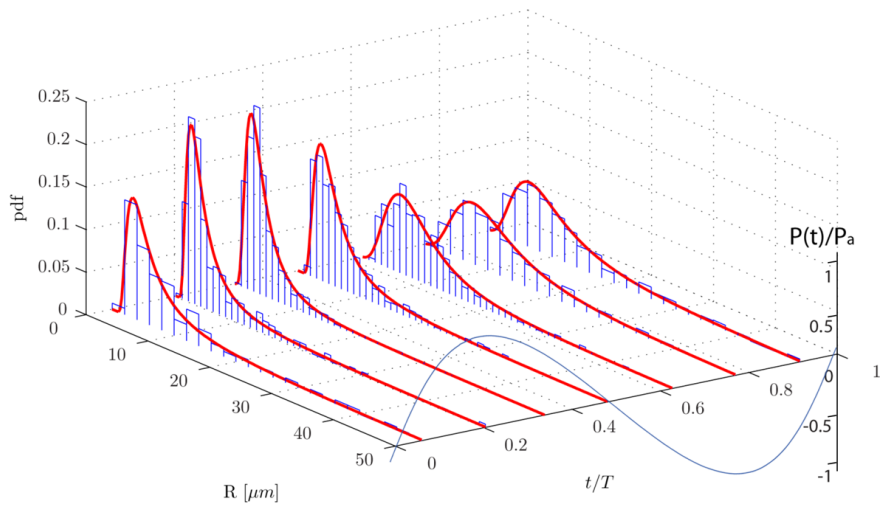

Figure 6.11: Bubble size distribution histograms at a power of $0.981 \mathrm{~W}$ for three pits. The axis to the extreme right represents the normalized pressure for the acoustic cycle. 
of the ejected bubble are very dramatic and their shape is highly deformed. The equivalent radii, therefore, are less representative of the actual size of a corresponding spherical bubble. The number of bubbles can also be under-estimated since some large bubbles overlap and the post-processing code can consider two or a blob of several bubbles as a single one. Nevertheless it provides a qualitative measure of their number. A typical PDF for high power is depicted in Fig. 6.11.

The bubble size distribution for a certain acoustic phase is the distribution of equivalent radii of the total number of bubbles over the 10 frames taken at that phase. The average radius is obtained by averaging the radii of all the bubbles in the 10 frames and the most probable radius is that with most occurrences at that phase. For example, a bubble size distribution such as that in Fig. 6.11 for $\mathrm{t} / \mathrm{T}=0$ is right-skewed i.e. more frequent smaller equivalent radii (R). The most probable radius is $\sim 2 \mu \mathrm{m}$, but the average radius is larger, as the distribution includes a range of radii up to 35 $\mu \mathrm{m}$. See Fig. 6.12 for more details.
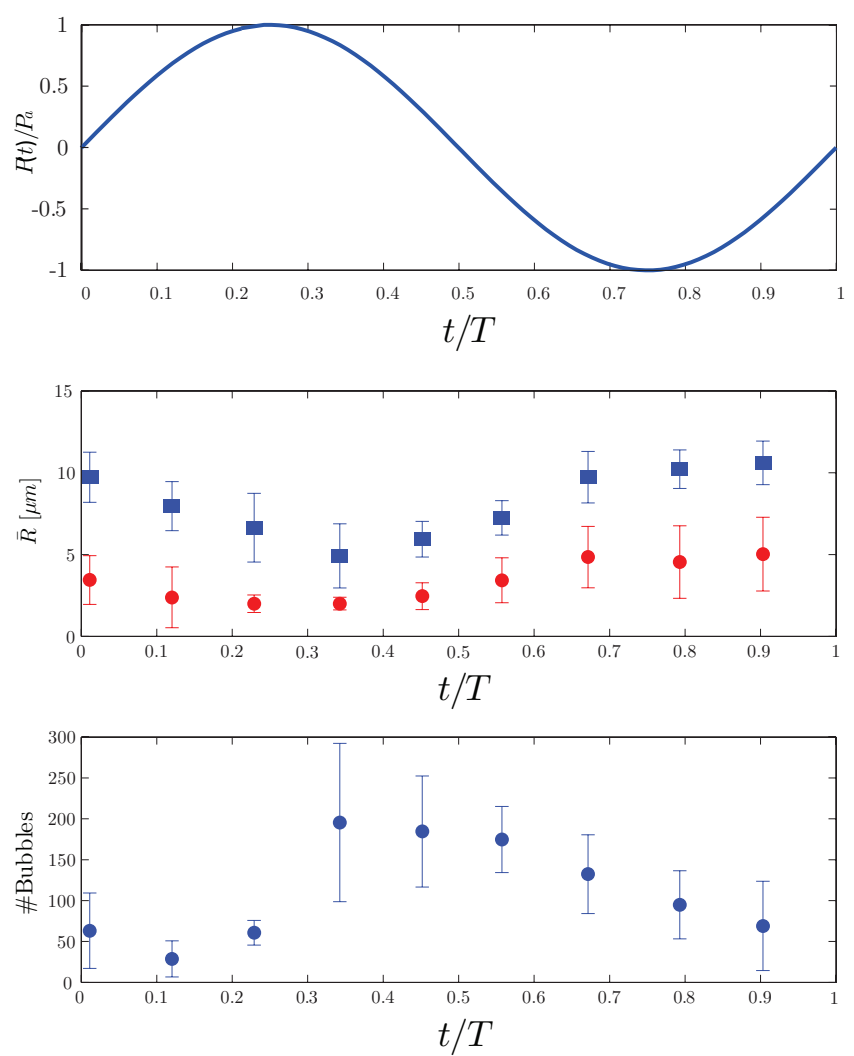

Figure 6.12: Top: Normalized pressure for the acoustic cycle. Center: Most probable radius (circles) and average radius (squares); Bottom: Average number of bubbles at different phases at a power of $0.981 \mathrm{~W}$ for three pits. 
Table 6.1 presents a compilation of the experimental data on several measures of bubble size and average number per cycle. As noted above, the radius of resonant bubbles at the experimental frequency is about $15 \mu \mathrm{m}$. The measured average bubble radius is of this order ( 8 to $16 \mu \mathrm{m}$ ) and the most probable radius is between 2 and 5 $\mu \mathrm{m}$. Figure 6.12 shows an example of the evolution of the most probable and average radii, together with the number of bubbles during one acoustic cycle at $0.981 \mathrm{~W}$ for three pits. It is seen in the bottom panel of this figure that the number of bubbles peaks shortly after the maximum pressure. This feature may reflect the expansion of the smaller bubbles which are below the optical resolution of our apparatus during the rest of the acoustic cycle.

Table 6.1: Bubble population characteristics in a cycle.

\begin{tabular}{cccc}
\hline Low power $(0.194 \mathrm{~W})$ & & & \\
& 1 pit & 2 pits & 3 pit \\
\hline Most probable radius $[\mu \mathrm{m}]$ & 17 & 3 & 2 \\
Average bubble radius $[\mu \mathrm{m}]$ & 16 & 10 & 12 \\
$N_{\text {bubble }}$ & 3 & 5 & 17 \\
\hline Medium power $(0.654 \mathrm{~W})$ & & & \\
& 1 pit & 2 pits & 3 pit \\
\hline Most probable radius $[\mu \mathrm{m}]$ & 2 & 3 & 3 \\
Average bubble radius $[\mu \mathrm{m}]$ & 9 & 12 & 8 \\
$N_{\text {bubble }}$ & 4 & 29 & 113 \\
\hline High power $(0.981 \mathrm{~W})$ & & & \\
& 1 pit & 2 pits & 3 pit \\
\hline Most probable radius $[\mu \mathrm{m}]$ & 2 & 3 & 5 \\
Average bubble radius $[\mu \mathrm{m}]$ & 8 & 9 & 10 \\
$N_{\text {bubble }}$ & 50 & 102 & 96 \\
\hline
\end{tabular}

The average number of bubbles instantaneously present is shown in Fig. 6.13 These data were obtained by summing the number of all bubbles visible in all frames (10 frames each for 10 phases) and dividing by a hundred. For the one-pit configuration, as the power is increased the number of bubbles increases as would be expected. The results shown for low and medium power correspond to isolated bubble clouds while for two and three pits at high power the clouds have merged as explained before. When this happens, the observed bubble number does not increase, possibly due to bubble-bubble interactions and the finite amount of gas available [52-54].

\subsubsection{Experimental radical production rates}

It was shown earlier [29] (Chapter 3) that the duration of the experiment does not seem to affect the reaction rates up to 30 minutes of operation. The radical produc- 


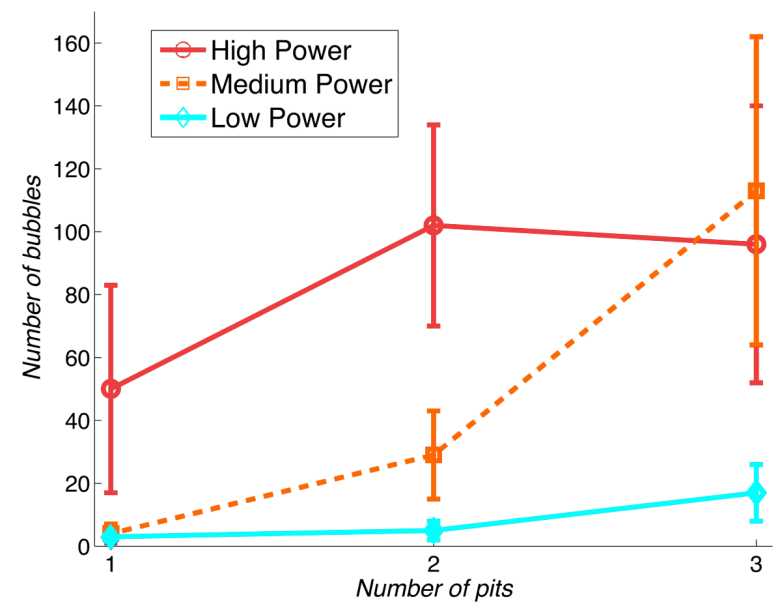

Figure 6.13: Average number of bubbles in the cavitation field for the different power settings: Low (0.194 W), Medium (0.654 W) and High $(0.981$ W).

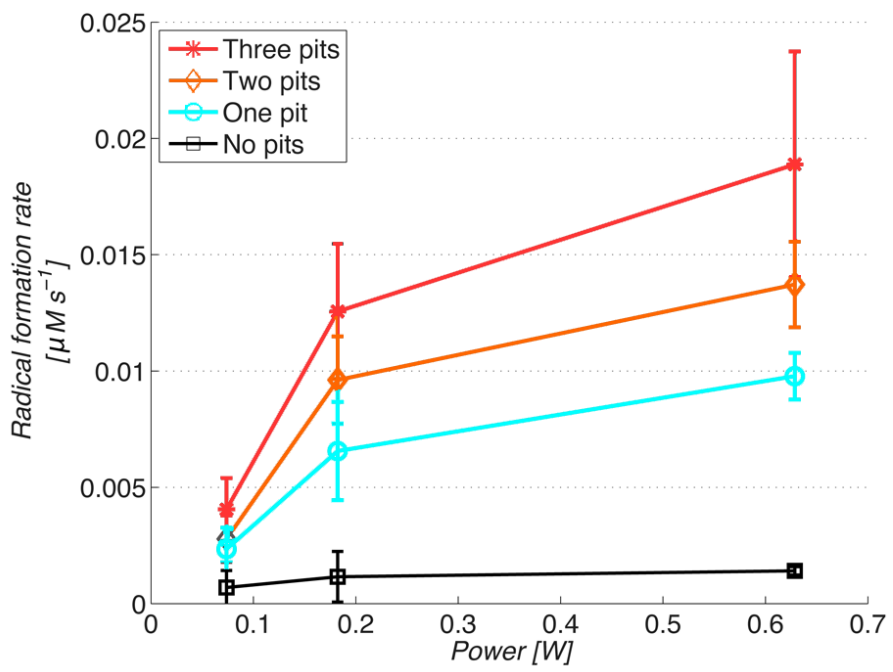

Figure 6.14: Radical production rates for the different power settings.

tion rate defined in Equation 6.1 is shown as a function of the ultrasonic power in Fig. 6.14. It is important to note that there is no significant effect of the US power when no pits are present. It is clear that the presence of the pits is essential for for the generation of sonochemically active bubbles: the pits enhance cavitation by facilitating the appearance of a larger number of bubbles, at a location in the reaction cell that otherwise would have no detectable bubbles or none at all.

For the lowest power the total radical production rates are around $5 \mathrm{nM} \mathrm{s}^{-1}$, but 
as the power is increased, the reaction rate increases as well. The maximum value corresponds to the three-pits configuration at high power.

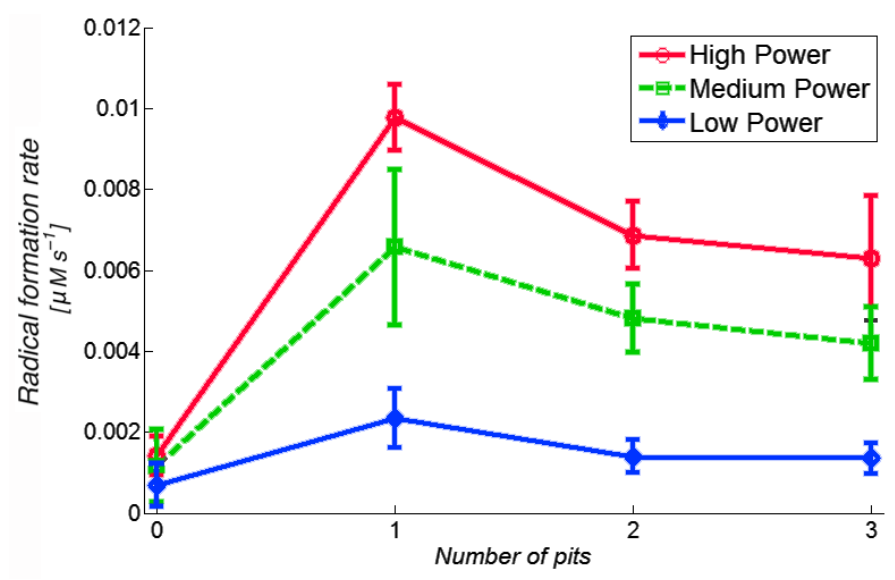

Figure 6.15: Radical production rates for the different power settings (Low 0.074 W, Medium $0.182 \mathrm{~W}$ and High $0.629 \mathrm{~W}$ ) divided by the number of pits.

When the amount of radicals formed per second is divided by the number of pits we see an interesting trend depicted in Fig. 6.15. For one pit the rate of radical formation is higher than for two- and three-pits in all cases. Possible factors contributing to this result are the mutual shielding of the bubbles, a detuning of the cell and change in the sound power distribution due to the larger gas volume fraction, the availability of dissolved gas in the liquid and possibly others.

If we calculate the radical production per cycle and divide it by the average number of bubbles per cycle, we can get an estimate to be compared with single bubble radical production measured by Didenko and Suslick [6] who reported data for a single bubble of maximum radius of $28.9 \mu \mathrm{m}$ driven at $52 \mathrm{kHz}$. They measured an $\mathrm{OH}$ radical generation rate of $6.6 \times 10^{5}$ per cycle. Our data, which are shown in Table 6.2, are of the same order of magnitude despite the differences in the experimental parameters. We expected lower rates due to the smaller volume of our bubbles (itself a consequence of the higher frequency), to mutual shielding and bubble deformation. A possible explanation for our results is radical production by bubbles smaller than $0.5 \mu \mathrm{m}$, which our experimental technique cannot resolve.

\subsubsection{Discussion of the calculated efficiencies}

In principle, a measure suitable for the quantification of the sonochemical conversion could be defined as:

$$
X_{U S}=\frac{\Delta H\left(\Delta N_{\text {rad }} / \Delta t\right)}{P_{U S}},
$$


Table 6.2: Radical production per detected bubble per cycle

\begin{tabular}{cccc}
\hline Low power & & & \\
$(0.074-0.194 \mathrm{~W})$ & 1 pit & 2 pits & 3 pit \\
& $7.06 \times 10^{5}$ & $5.03 \times 10^{5}$ & $2.15 \times 10^{5}$ \\
\hline Medium power & & & \\
$(0.182-0.654 \mathrm{~W})$ & 1 pit & 2 pits & 3 pit \\
& $1.48 \times 10^{6}$ & $2.99 \times 10^{5}$ & $1.00 \times 10^{5}$ \\
\hline High power & & & \\
$(0.629-0.981 \mathrm{~W})$ & 1 pit & 2 pits & 3 pit \\
& $1.76 \times 10^{5}$ & $1.21 \times 10^{5}$ & $1.78 \times 10^{5}$ \\
\hline
\end{tabular}

where $P_{U S}$ is the power absorbed by the transducer and $\Delta H$ is the energy required for the formation of $\mathrm{OH}$ radicals $\left(5.1 \mathrm{eV}=8.2 \times 10^{-19} \mathrm{~J}\right)$, which is equal to the enthalpy of formation associated with the following chemical reaction:

$$
\mathrm{H}_{2} \mathrm{O} \stackrel{\Delta H=-5.1 \mathrm{eV}}{\rightarrow} \mathrm{OH}+\mathrm{H}^{\cdot}
$$

The problem with this definition is that only a small fraction of the power provided to the piezo is responsible for the sonochemical effect [55]. As a mater of fact, by comparing the power absorbed with and without pits and, therefore, in the presence or absence of intense bubble activity, we have found very small differences. This leads us to conclude that the majority of the power absorbed by the system is lost in the electrical-to-mechanical conversion. Nevertheless we will use the definition (6.2) as a convenient dimensionless measure of the sonochemical conversion efficiency. The electric power absorbed by the transducer $P_{U S}$ was determined from the measured voltage and current, and their phase difference.

The efficiency values calculated with Eq. 6.2 are shown in Fig. 6.16. As presented before [29], the most efficient setting appears to correspond to medium power. However, for the reasons mentioned before, the efficiencies shown in the figure may not reflect purely acoustic features of the system. Nevertheless we present this data following the prevailing custom in the sonochemical literature [27, 55].

\subsubsection{Obtaining numerical effective bubble size distributions and effec- tive driving pressures}

As explained in Section 6.2.5, one can estimate the production of radicals in a single bubble on the basis of existing models. In order to apply these models to the complex bubble fields studied in the present experiments, it is necessary to know the distribution of bubble radii and the effective pressure amplitude to which each bubble is subjected. 


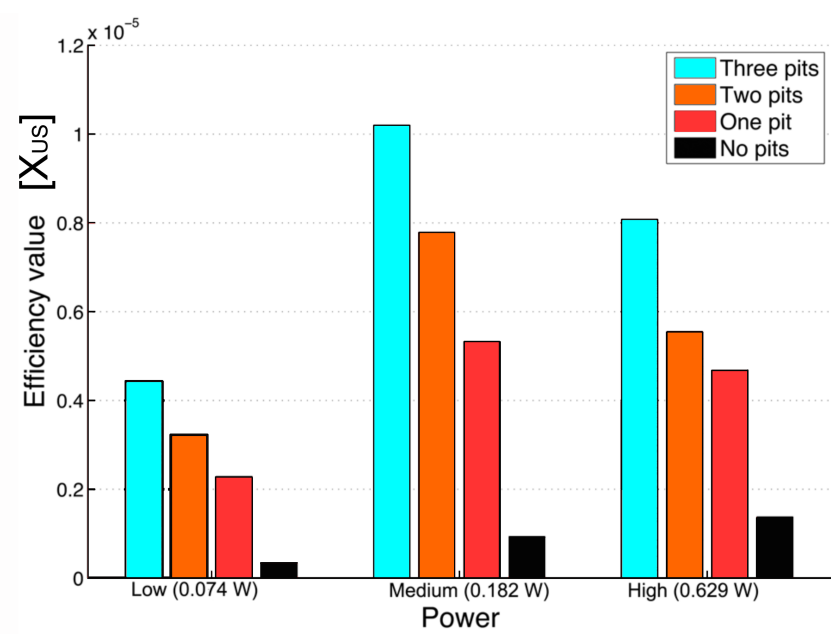

Figure 6.16: Nominal experimental efficiency values $\left(X_{U S}\right)$ for different number of pits and different US powers calculated from Eq. 6.2. The presence of pits for each power makes a clear difference with an increase in efficiency as the number of pits is increased. In the case of no pits, an increase in power produces a slow increase in efficiency. From low to medium power the trends increase for any number of pits. For the high power case, there appears to be a decrease in efficiency for any number of pits. It is not clear whether this result corresponds to true differences in the acoustic and sonochemical processes or to a decreased efficiency of conversion from electrical to mechanical power.

For this purpose we relied on measured probability distributions of bubble sizes at each phase of the sound field. These PDF were obtained by collecting 10 snapshots of the bubble field for each phase of the pressure. Each snapshot can be considered as statistically equivalent and independent from the others as the separation between successive ones was $2 \times 10^{5}$ cycles which is much longer than the expected lifetime of the individual bubbles. The idea of the simulation was to determine a pressure amplitude and bubble equilibrium radius distribution that matched the measured radii distribution at each phase.

We started from the measured distribution at a particular phase chosen so as to have a large number of bubbles, which generally coincided with a small average radius and small standard deviation. For each one of these bubble radii we integrated the extended Rayleigh-Plesset equation assuming a vanishing initial radial velocity and using different pressure amplitudes [38]. We found that the nominal pressure amplitudes applied in the experiment produced bubble size distributions quite different from the measured ones, as shown in Fig. 6.17.

In order to match the measured distributions (see Figs. 6.9, 6.10 and 6.11) it was necessary to adjust the pressure amplitudes used in the simulations, which resulted in much lower values than the nominal experimental ones. The adjustment was done by 

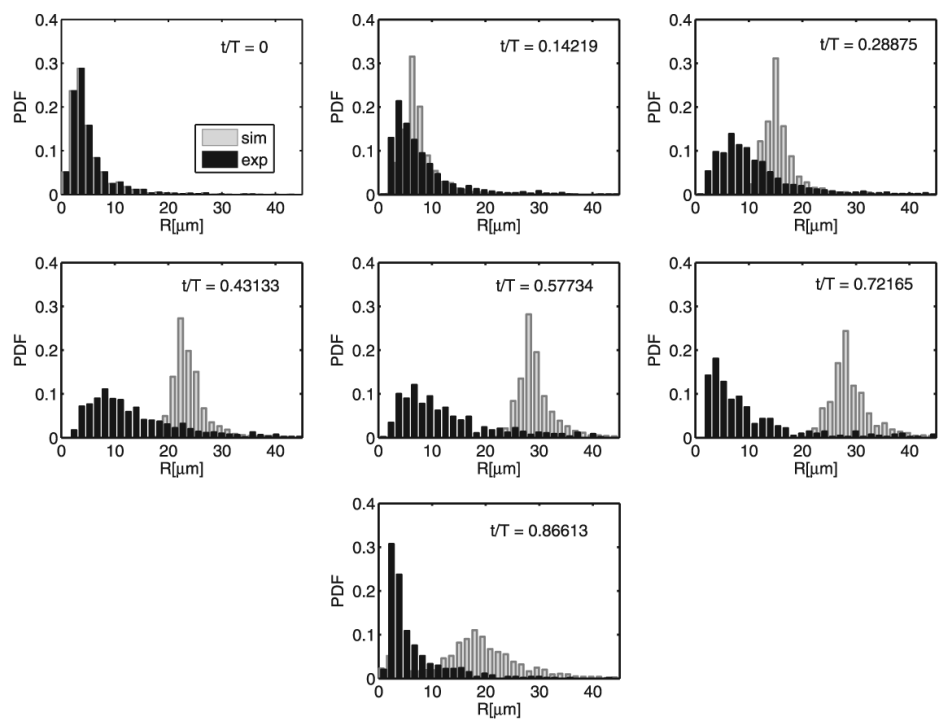

Figure 6.17: Bubble size distribution from experiment (black) and simulation (grey), for high power $(0.981 \mathrm{~W})$ and the three-pit configuration. It appears that the pressure amplitude measured with the hydrophone overestimated the effective value felt by the bubble population. Simulations carried out using these values were not able to describe the evolution in time of the radii distribution, as the average radius was overestimated during the whole cycle, the expansion phase was much longer than in experiments and the distributions were almost symmetric, instead of right-skewed.

minimizing the cumulative squared difference between experimental and calculated PDF for each phase (see Fig. 6.18).

The pressure levels deduced in this way were in the range between $110 \mathrm{kPa}$ and $170 \mathrm{kPa}$. Since the minimum radius detectable in the experiment was about $0.5 \mu \mathrm{m}$, a larger number of small bubbles appeared in simulations than in the experiment. The fit between experimental and calculated PDF was best for high applied power and more pits, i.e. for high numbers of nucleated bubbles (see Fig. 6.19 and 6.20).

At low applied power the number of bubbles detected in the experiments was too small to provide good statistics, especially in the one-pit configuration. Moreover, at low power, the standard deviations of both the experimental and the calculated PDF normalized by the average radii were higher during the whole oscillation cycle, even if the average radii themselves were larger. This means that the data were more scattered than in the high power case, both in absolute and relative terms.

In the one-pit case, if we increased the applied power, the effective pressure derived from simulations monotonically increased (see Fig. 6.21) together with the number of bubbles measured in the experiments, while their average radii and normalized standard deviations decreased. In the two- and three-pits cases, a similar 


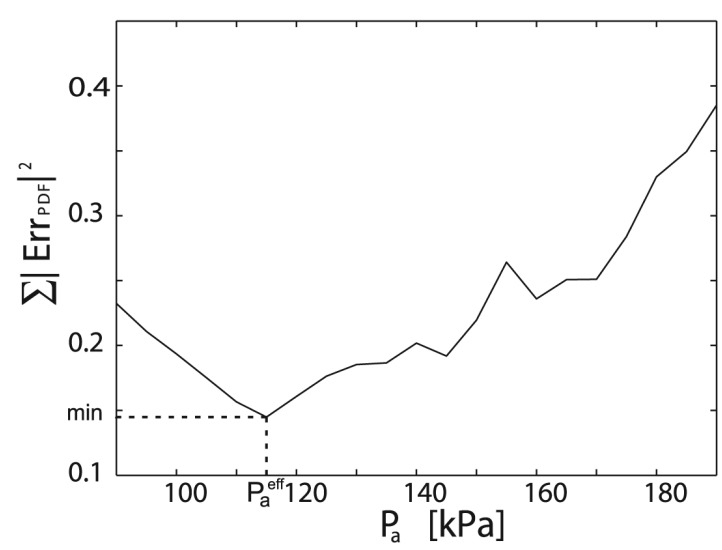

Figure 6.18: Cumulative squared error (over all bubble sizes and acoustic phases) between the PDF of the radii from experiments and from the simulations, as a function of the pressure values used in the simulations, for three-pit configuration, at high power $(0.981 \mathrm{~W})$. The effective driving pressure felt by the bubble population was $115 \mathrm{kPa}$ and was extracted by minimizing the cumulative squared error.

behavior was observed, with the effective pressure rising with the applied power and the average radius decreasing.

This trend, however, changed in correspondence of the pressure levels at which the observed bubble distribution switched from separate clouds surrounding each pit to the complex bubble field structure observed in Fig. 6.3 and Fig. 6.4. Beyond this threshold, the measured bubble size distribution changed significantly and the pressure necessary to fit the data abruptly decreased. This supports our view that the mutual shielding of the bubbles leads to a reduction in the effective pressure seen by the bubbles.

\subsubsection{Calculation of the radical production}

On the basis of the bubble sizes and pressure amplitudes determined in the way described in Subsection 6.3.5, we calculated then the number of $\mathrm{OH}$. radicals produced by singles bubble with the equilibrium radius corresponding to the experimental distribution.

The bubble population at the instant at which the average radius over the 10 frames was minimum was taken as the initial and equilibrium distribution for the simulations. This generally coincided with the instant when the highest number of bubbles was found.

For this calculation, one hundred equi-spaced bubble equilibrium radii were chosen for each $10 \mu \mathrm{m}$ size bin. For the pressure conditions given by the fitting pro- 

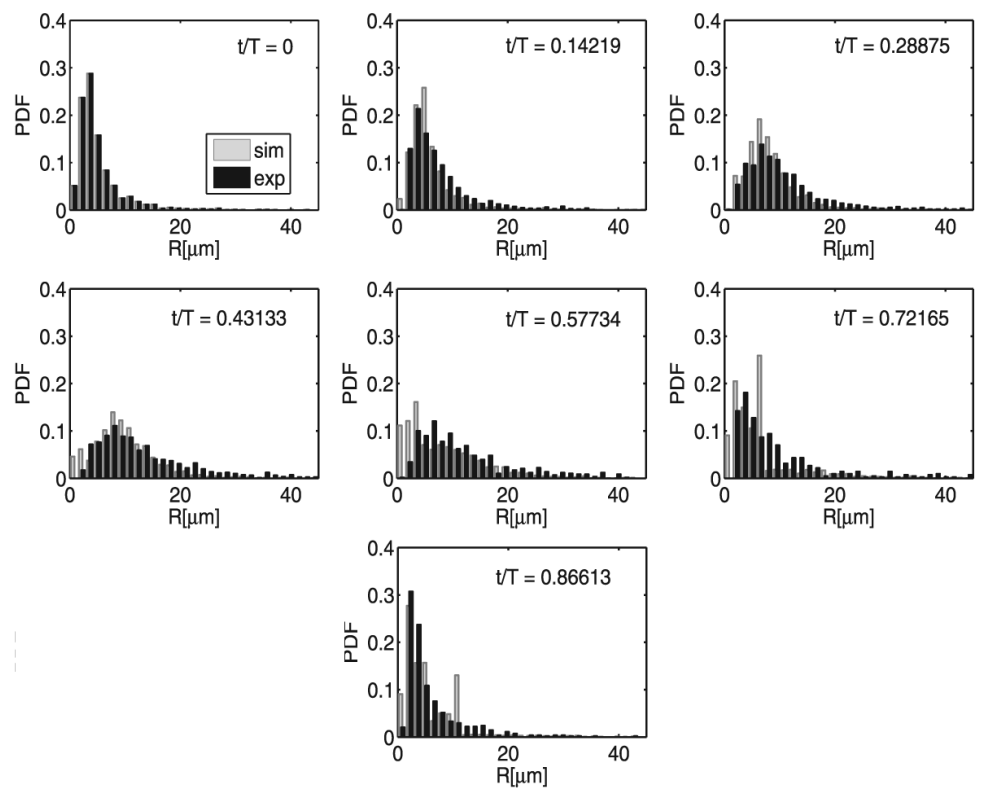

Figure 6.19: Bubble size distribution from experiment (black) and simulation (grey), for high power $(0.981 \mathrm{~W})$ and the three-pit configuration. The effective pressure amplitude extracted of $115 \mathrm{kPa}$ provided a good description of the dynamics of the system. The agreement between experiments and simulations was best for more pits and high applied power, i.e. for high number of nucleated bubbles. As expected, a higher number of small sized bubbles appeared in simulations than in experiments, as the minimum bubble radius that the experimental apparatus could detect was $0.5 \mu \mathrm{m}$.

cedure, the number of radicals diffusing inside the liquid over the first cycle was calculated for each value of the radius. The results were then averaged over all the radii belonging to the same bin.

As a general behavior, the calculated chemical output was higher at higher effective pressure but, in the two- and three-pits configurations, the radical production exhibited a significant dependence on the bubble size distribution, which was different before and after the transition from individual bubble clouds to complex patterns. Thus, a higher radical production was observed in the latter cases even for the same calculated effective pressure (see Fig. 6.22).

The transition to the complex bubbles patterns affects the acoustic field and the liquid flow in the cell, with possible consequences for the sonochemical activity as found in earlier studies [56].

In all the cases examined the whole $\mathrm{OH} \cdot$ radical production was found to come from bubbles smaller than resonance size, i.e. smaller than $15 \mu \mathrm{m}$, and mainly from those with a radius around $5 \mu \mathrm{m}$ (see Fig. 6.23, 6.24, 6.25). In particular, higher 

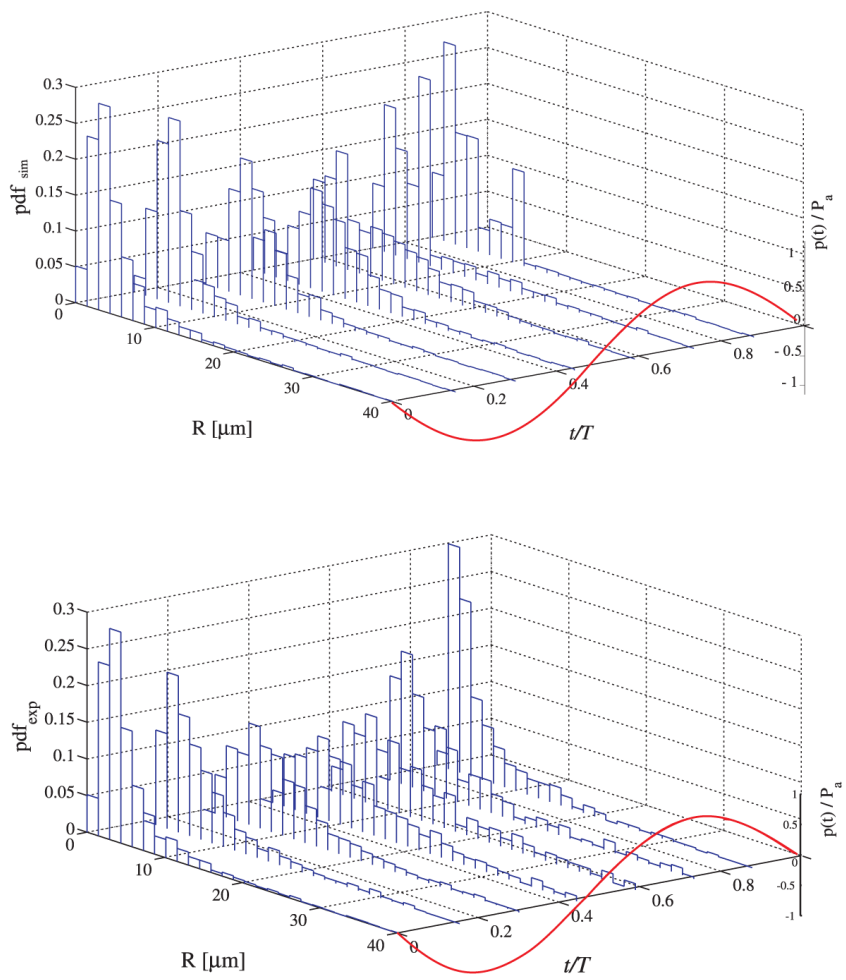

Figure 6.20: Bubble size distribution histograms at high power $(0.981 \mathrm{~W})$ for three pits. From experiments (lower figure), and from simulations (upper figure) with the deduced effective pressure (115 kPa), which gives the best fit to the observed bubble distribution.

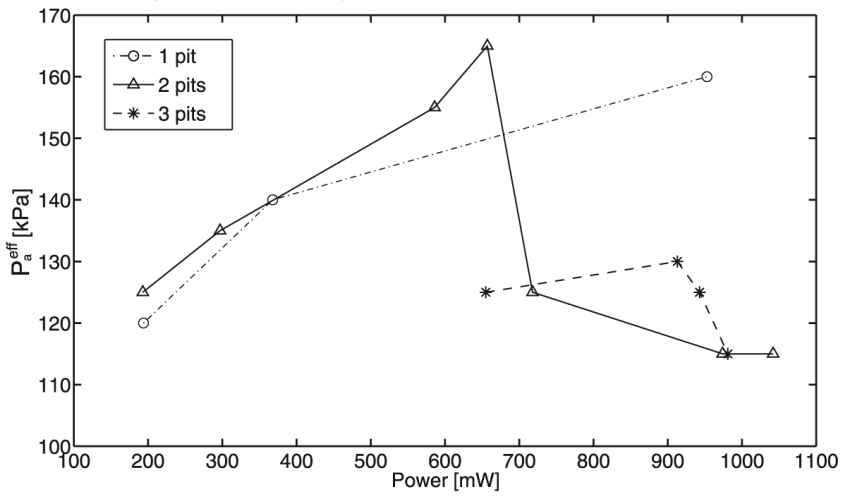

Figure 6.21: Effective driving pressure felt by the bubble population as a function of the applied power. These values were derived from our fitting procedure between experimental and calculated PDF of the radii (see Fig. 6.18). For different number of pits, bubbles started to appear for different applied powers in experiments, although corresponding to the same deduced effective pressure (around $125 \mathrm{kPa}$ ). In the one-pit configuration, the effective pressure monotonically grew with the electric power. In the two-and three-pit cases the effective pressure initially had a similar behavior, but it abruptly decreased when the bubbles started to migrate towards the center point of the pit array above $0.657 \mathrm{~W}$ and $0.914 \mathrm{~W}$ respectively. 

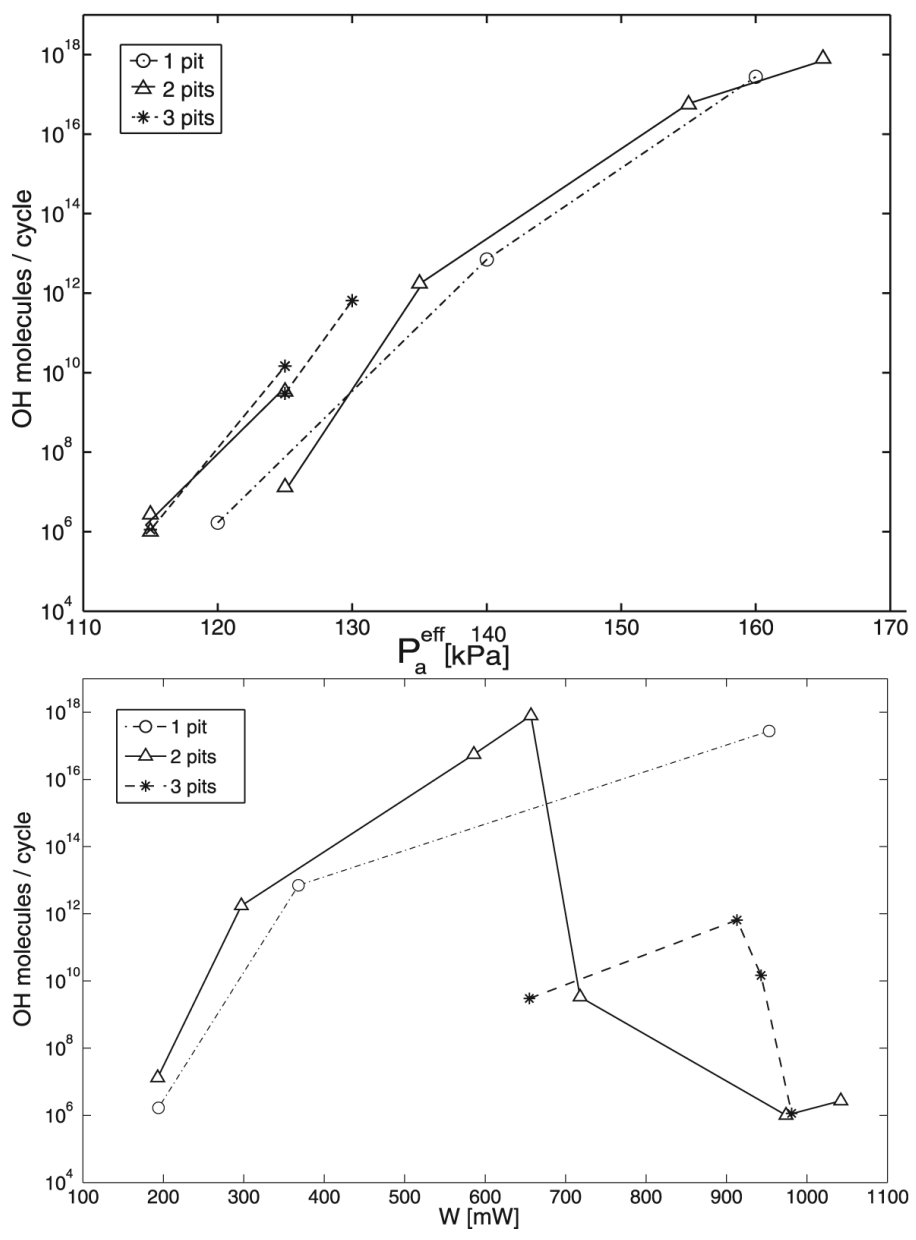

Figure 6.22: Number of $\mathrm{OH}$ - radicals diffusing into the liquid, in each acoustic cycle, as a function of pressure (upper figure) and applied power (lower figure), both calculated from simulations. The calculated chemical production increased with the effective pressure, but also depended on the PDF of the radii. In the two-and three-pit cases, when the bubbles migrated towards the center point of the pit array (at $0.657 \mathrm{~W}$ and $0.914 \mathrm{~W}$ ), more radicals were produced than when they remain near the pits, although the effective pressure was the same $(125 \mathrm{kPa})$. In the upper figure this corresponds to the first branch of the two-and three-pit curves (below $125 \mathrm{kPa}$ ). The right branches correspond to the initial rise of the power. 
effective pressure amplitudes resulted in smaller sizes of the bubbles giving the peak chemical production. This effect is dominant with respect to differences in bubble volume and the surface area of the bubble across which radicals diffuse.
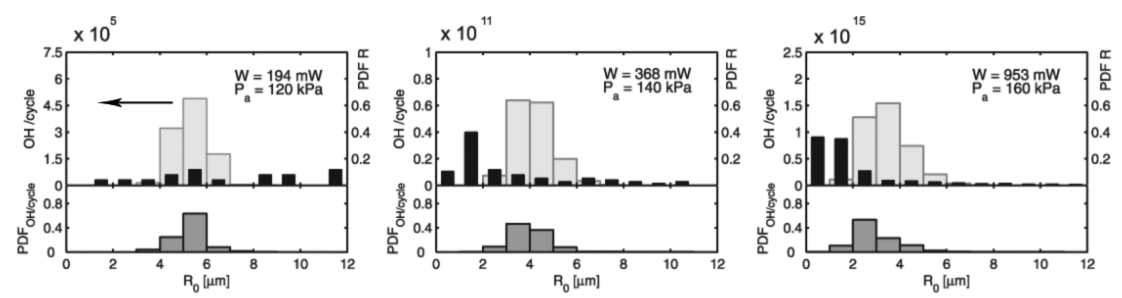

Figure 6.23: One-pit case. Upper figures, light grey: calculated number of $\mathrm{OH} \cdot$ radicals diffusing into the liquid at each acoustic cycle per bubble, as a function of the initial radius $R_{0}$ for a single bubble (see Section 6.3.6 for the details). Upper figures, black: PDF of the initial experimental distributions of the radii in the one-pit case, for different applied powers. Lower figures, dark grey: PDF of the OH. radicals diffusing into the liquid in each acoustic cycle, as a function of the initial radius. These values were obtained by normalizing the number of the OH/cycle produced by every bin of initial radii over the total number of $\mathrm{OH} / \mathrm{cycle}$ from the specific distribution. These lower figures can be seen as a combination of the two upper graphs. Simulations showed that the most radical production came from bubbles smaller than resonant size $(15 \mu \mathrm{m})$. In particular, higher effective pressure values corresponded to smaller sizes of the most active bubbles.

The experimental rates span over one decade, while the theoretical rates shown in Figure 6.22 span six decades. The reason for this is that the experimental precision is much lower than that of the numerical calculations, hence the strength of combining these two approaches.

It must be noted that our spherical bubble model overestimates the reaction rates measured in the experiments. This is perhaps not surprising given that the many factors that induce non-spherical collapse and therefore limit the compression of the gas are absent from the simulation. The simulations should be regarded as establishing an upper limit to the reaction rates. In principle, it may be thought that non-spherical bubble models could reduce the gap between simulations and experiments, although the complexity of the experimental situation will probably lie beyond any practical model in the foreseeable future. 

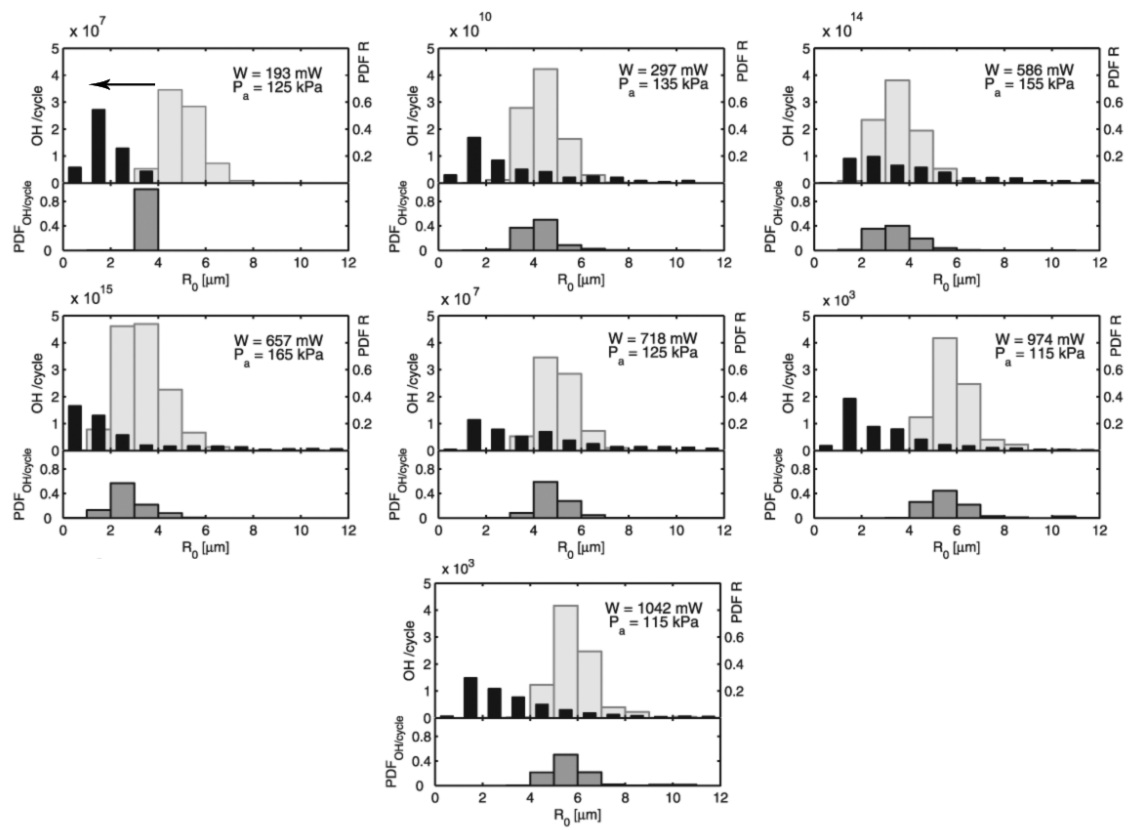

Figure 6.24: Same as Fig. 6.23 for the two-pit case.
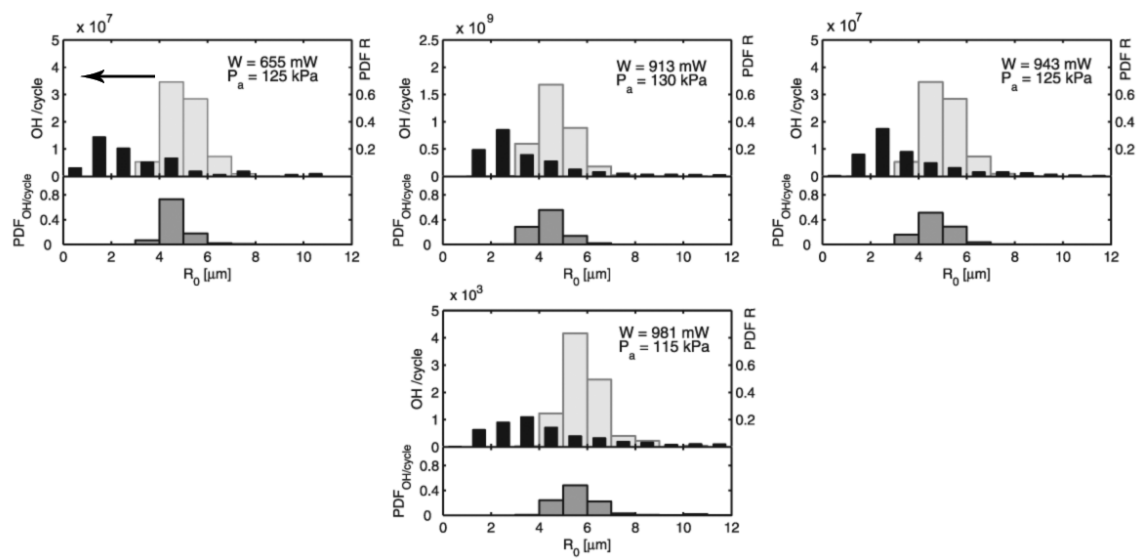

Figure 6.25: Same as Fig. 6.23 for the three-pit case. 


\subsection{Summary and Conclusions}

We achieved a more controllable and more efficient sonoreactor design by microfabricating small pits on a silicon chip immersed in a small sonoreactor of the ultrasonic bath type. Under the action of an ultrasonic field, the air trapped in the pits gives rise to a stream of small bubbles which are responsible for the intense sonochemical activity. A rectified diffusion process is responsible for replenishing the air and maintaining the activity of the pit.

We have shown that this modification of the solid surface yields considerably higher conversion rates. We have measured the bubble size distribution at different power settings and characterized its evolution during the acoustic cycle.

Simulations were carried out in order to give an estimate of the radical production in the different power settings. By fitting the experimental and the calculated PDF of the radii and minimizing the cumulative squared differences between the two, an estimate of the effective forcing pressure felt by the bubbles was found. In the two- and three-pit cases the effective pressure dropped when bubbles started to migrate towards the center point of the pit array. Simulations also allowed us to give a description of the temporal evolution of the smaller bubbles below $3 \mu$ m radius, compatible with the experimental PDF. This turned out to be particularly relevant, as calculations showed that the most $\mathrm{OH}$ - radical production came from the smaller bubbles around $5 \mu \mathrm{m}$ radius. As a general trend, the calculated chemical rate increased monotonically with the effective pressure.

Even though the numerical model considers spherical bubbles without interaction, an important strength lies in the possibility of taking the actual experimental bubble size distribution (with its equivalent radius corresponding to non-spherical bubbles) and giving information on the smallest bubbles that are below the experimental observability. This computation can be considered as a useful complement to the experiment since it permits one to estimate the local pressure value to which the bubbles are exposed and which cannot be directly measured with the available pressure sensors.

It may be possible to extend the present surface modification to larger scale sonoreactors by supplying similar nucleation sites on larger surfaces and in a much greater number. As a guide towards the development and optimization of these types of reactors, it will be useful to investigate in greater detail several aspects such as pit density, dimensions, mutual interaction and many others.

\section{Acknowledgements}

We acknowledge S. Schlautmann (microfabrication) and J. Martinez (image processing) for their technical support and also Prof. F. Grieser and Prof. M. Ashokkumar 
for fruitful discussions. The advices from Prof. C.-D. Ohl and Dr. Tandiono on the experimental pressure measurements is highly appreciated. This research was supported by the Technology Foundation STW, Applied Science Division of NWO and the Technology Programme of the Ministry of Economic Affairs, The Netherlands.

\section{References}

[1] M. Ashokkumar, T. J. Mason, Sonochemistry, John Wiley \& Sons, Inc., 2000.

[2] A. Gedanken, Sonochemistry and its application to nanochemistry, Curr. Sci. 85 (12) (2003) 1730-1734.

[3] N. Mahamuni, Y. Adewuyi, Advanced oxidation processes (AOPs) involving ultrasound for waste water treatment: A review with emphasis on cost estimation, Ultrason. Sonochem. 17 (6) (2010) 990-1003.

[4] M. Ashokkumar, D. Sunartio, S. Kentish, R. Mawson, L. Simons, K. Vilkhu, C. Versteeg, Modification of food ingredients by ultrasound to improve functionality: A preliminary study on a model system, Innov. Food Sci. Emerg, Technol. 9 (2) (2008) 155-160.

[5] D. Lohse, Sonoluminescence-Cavitation hots up, Nature 434 (2005) 33-34.

[6] Y. T. Didenko, K. S. Suslick, The energy efficiency of formation of photons, radicals and ions during singlebubble cavitation, Nature 418 (6896) (2002) 394-397.

[7] U. Parlitz, R. Mettin, S. Luther, I. Akhatov, M. Voss, W. Lauterborn, Spatio-temporal dynamics of acoustic cavitation bubble clouds, Phil. Trans. R. Soc. Lond. A 357 (1751) (1999) 313.

[8] J. Appel, P. Koch, R. Mettin, D. Krefting, W. Lauterborn, Stereoscopic high-speed recording of bubble filaments, Ultrason. Sonochem. 11 (1) (2004) 39-42.

[9] M. P. Brenner, S. Hilgenfeldt, D. Lohse, Single-bubble sonoluminescence Rev. Mod. Phys. 74 (2002) $425-$ 484.

[10] K. S. Suslick, D. J. Flannigan, Inside a collapsing bubble: Sonoluminescence and the conditions during cavitation, Annu. Rev. Phys. Chem. 59 (2008) 659-683.

[11] K. Suslick, N. Eddingsaas, D. Flannigan, S. Hopkins, H. Xu, Extreme Conditions during Multibubble Cavitation: Sonoluminescence as a Spectroscopic Probe, Ultrason. Sonochem. 18 (2011) 842 - 846.

[12] R. Pflieger, H. Brau, S. Nikitenko, Sonoluminescence from $\mathrm{OH}(\mathrm{C} 2 \Sigma+)$ and $\mathrm{OH}(\mathrm{A} 2 \Sigma+)$ Radicals in Water: Evidence for Plasma Formation during Multibubble Cavitation, Chem. Eur. J. 16 (39) (2010) 11801-11803.

[13] F. Burdin, N. Tsochatzidis, P. Guiraud, A. Wilhelm, H. Delmas, Characterisation of the acoustic cavitation cloud by two laser techniques, Ultrason. Sonochem. 6 (1-2) (1999) 43-51.

[14] S. Luther, R. Mettin, P. Koch, W. Lauterborn, Observation of acoustic cavitation bubbles at 2250 frames per second, Ultrason. Sonochem. 8 (3) (2001) 159-162.

[15] N. Tsochatzidis, P. Guiraud, A. Wilhelm, H. Delmas, Determination of velocity, size and concentration of ultrasonic cavitation bubbles by the phase-Doppler technique, Chem. Eng. Sci. 56 (5) (2001) 1831-1840.

[16] S. Labouret, J. Frohly, Bubble size distribution estimation via void rate dissipation in gas saturated liquid. Application to ultrasonic cavitation bubble fields, Eur. Phys. J. AP 19 (01) (2002) 39-54.

[17] W. Chen, T. Matula, L. Crum, The disappearance of ultrasound contrast bubbles: observations of bubble dissolution and cavitation nucleation, Ultrasound Med. Biol 28 (6) (2002) 793-803. 
[18] D. Sunartio, M. Ashokkumar, F. Grieser, Study of the coalescence of acoustic bubbles as a function of frequency, power, and water-soluble additives, J. Am. Chem. Soc. 129 (18) (2007) 6031-6036.

[19] A. Brotchie, F. Grieser, M. Ashokkumar, Effect of power and frequency on bubble-size distributions in acoustic cavitation, Phys. Rev. Lett. 102 (8) (2009) 84302.

[20] C. E. Brennen, Cavitation and Bubble Dynamics, Oxford University Press, Oxford, 1995.

[21] T. G. Leighton, The acoustic bubble, Academic Press, London, 1994.

[22] M. Ashokkumar, The Characterization of Acoustic Cavitation Bubbles-An Overview, Ultrason. Sonochem. 2010 .

[23] R. Tronson, M. Ashokkumar, F. Grieser, Comparison of the effects of water-soluble solutes on multibubble sonoluminescence generated in aqueous solutions by 20 -and $515-\mathrm{kHz}$ pulsed ultrasound, J. Phys. Chem. B 106 (42) (2002) 11064-11068.

[24] K. Okitsu, T. Suzuki, N. Takenaka, H. Bandow, R. Nishimura, Y. Maeda, Acoustic multibubble cavitation in water: A new aspect of the effect of a rare gas atmosphere on bubble temperature and its relevance to sonochemistry, J. Phys. Chem. B 110 (41) (2006) 20081-20084.

[25] T. Tuziuti, K. Yasui, Y. Iida, Spatial study on a multibubble system for sonochemistry by laser-light scattering, Ultrason. Sonochem. 12 (1-2) (2005) 73-77.

[26] S. Hatanaka, K. Yasui, T. Kozuka, T. Tuziuti, H. Mitome, Influence of bubble clustering on multibubble sonoluminescence, Ultrasonics 40 (1-8) (2002) 655-660.

[27] M. W. A. Kuijpers, M. F. Kemmere, J. T. F. Keurentjes, Calorimetric study of the energy efficiency for ultrasound-induced radical formation, Ultrasonics 40 (1-8) (2002) 675-678.

[28] Z. Zeravcic, D. Lohse, W. van Saarloos, Collective oscillations in bubble clouds, J. Fluid Mech. 680 (2011) $114-149$.

[29] D. Fernandez Rivas, A. Prosperetti, A. G. Zijlstra, D. Lohse, H. J. G. E. Gardeniers, Efficient sonochemistry through microbubbles generated with micromachined surfaces, Angew. Chem. Int. Ed. 49 (50) (2010) 96999701.

[30] N. Bremond, M. Arora, C. D. Ohl, D. Lohse, Controlled multi-bubble surface cavitation, Phys. Rev. Lett. 96 (2006) 224501.

[31] N. Bremond, M. Arora, S. M. Dammer, D. Lohse, Interaction of cavitation bubbles on a wall, Phys. Fluids 18 (12) (2006).

[32] P. Marmottant, J. P. Raven, H. Gardeniers, J. G. Bomer, S. Hilgenfeldt, Microfluidics with ultrasound-driven bubbles, J. Fluid Mech. 568 (2006) 109-118.

[33] B. M. Borkent, S. Gekle, A. Prosperetti, D. Lohse, Nucleation threshold and deactivation mechanisms of nanoscopic cavitation nuclei, Phys. Fluids 21 (10) (2009).

[34] A. van der Bos, A. Zijlstra, E. Gelderblom, M. Versluis, ilif: illumination by laser-induced fluorescence for single flash imaging on a nanoseconds timescale, Exp. Fluids 51 (2011) 1283-1289.

[35] F. Meyer, Topographic distance and watershed lines, Signal Process. 38 (1994) 113-125.

[36] T. J. Mason, J. P. Lorimer, D. M. Bates, Y. Zhao, Dosimetry in sonochemistry - the use of aqueous terephthalate ion as a fluorescence monitor, Ultrason. Sonochem. 1 (2) (1994) S91-S95.

[37] Y. Iida, K. Yasui, T. Tuziuti, M. Sivakumar, Sonochemistry and its dosimetry, Microchem. J. 80 (2) (2005) 159-164. 
[38] A. Prosperetti and A. Lezzi, Bubble dynamics in a compressible liquid. part 1. First-order theory, J. Fluid Mech. 168 (1986) 457-478.

[39] R. Toegel, S. Hilgenfeldt, D. Lohse, Suppressing dissociation in sonoluminescing bubbles: The effect of excluded volume, Phys. Rev. Lett. 88 (2002) 034301.

[40] R. Toegel, D. Lohse, Phase diagrams for sonoluminescing bubbles: A comparison between experiment and theory, J. Chem. Phys. 118 (2003) 1863.

[41] L. Stricker, A. Prosperetti, D. Lohse, Validation of an approximate model for the thermal behavior in acoustically driven bubbles, J. Acoust. Soc. Am. 130 (5) (2011) 3243-3251.

[42] T. G. Leighton, Bubble population phenomena in acoustic cavitation, Ultrason. Sonochem. 2 (2) (1995) S123S136.

[43] E. A. Neppiras, Acoustic cavitation, Phys. Rep. 61 (3) (1980) 159-251.

[44] A. Zijlstra, Acoustic surface cavitation, Ph.D. thesis, University of Twente (2011).

[45] H. Gelderblom, A. Zijlstra, L. van Wijngaarden, A. Prosperetti, Oscillations of a gas pocket on a liquid-covered solid surface, Submitted to Phys. Fluids XX (XX) (2012) XX-XX.

[46] A. Moussatov, C. Granger, B. Dubus, Cone-like bubble formation in ultrasonic cavitation field, Ultrason. Sonochem. 10 (4-5) (2003) 191-195.

[47] A. Moussatov, R. Mettin, C. Granger, T. Tervo, B. Dubus, W. Lauterborn, Evolution of acoustic cavitation structures near larger emitting surface, in: Proc. 5th World Congress on Ultrasonics, Paris, France, 2003, pp. 955-958.

[48] R. Mettin, S. Luther, C. Ohl, W. Lauterborn, Acoustic cavitation structures and simulations by a particle model, Ultrason. Sonochem. 6 (1-2) (1999) 25-29.

[49] R. Pecha, B. Gompf, Microimplosions: Cavitation collapse and shock wave emission on a nanosecond time scale, Phys. Rev. Lett. 84 (2000) 1328-1330.

[50] M. P. Brenner, S. Hilgenfeldt, D. Lohse, Single bubble sonoluminescence, Rev. Mod. Phys. 74 (2002) $425-484$.

[51] N. Bremond, M. Arora, S. M. Dammer, D. Lohse, Interaction of cavitation bubbles on a wall, Phys. Fluids 18 (12) (2006) 121505

[52] S. Uzel, M. A. Chappell, S. J. Payne, Modeling the cycles of growth and detachment of bubbles in carbonated beverages, J. Phys. Chem. B 110 (14) (2006) 7579-7586.

[53] J. B. Fowlkes, L. A. Crum, Cavitation threshold measurements for microsecond length pulses of ultrasound, J. Acoust. Soc. Am. 83 (6) (1988) 2190-2201.

[54] L. Crum, Nucleation and stabilization of microbubbles in liquids, Appl. Sci. Res. 38 (1) (1982) 101-115.

[55] J. Berlan, T. J. Mason, Sonochemistry: from research laboratories to industrial plants, Ultrasonics 30 (4) (1992) $203-212$

[56] S. Hatanaka, H. Mitome, K. Yasui, S. Hayashi, Multibubble sonoluminescence enhancement by fluid flow, Ultrasonics 44 (2006) e435-e438. 


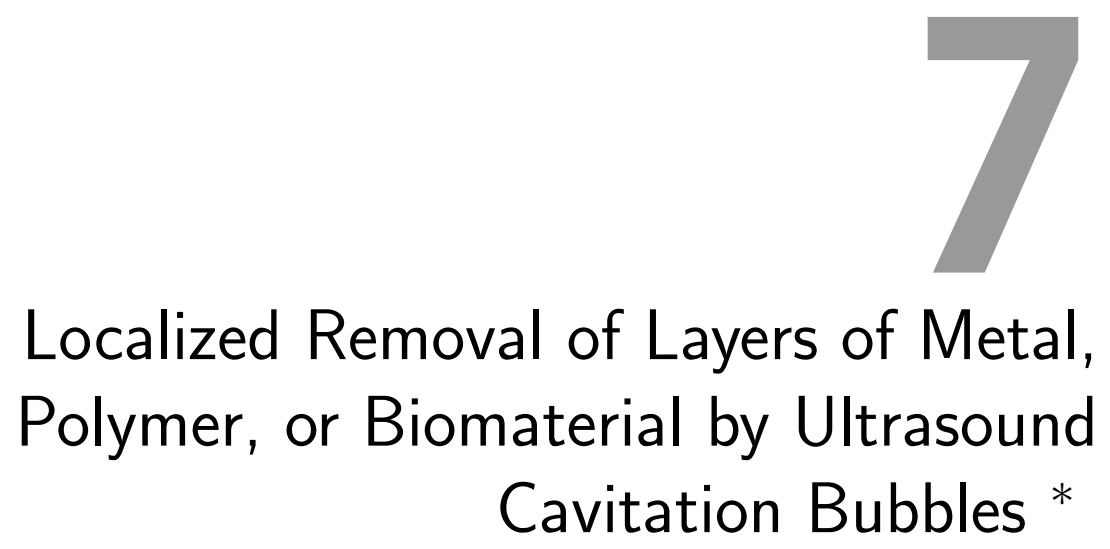

\begin{abstract}
We present an ultrasonic device with the ability to locally remove deposited layers from a glass slide in a controlled and rapid manner. The cleaning takes place as the result of cavitating bubbles near the deposited layers and not due to acoustic streaming. The bubbles are ejected from air-filled cavities micromachined in a silicon surface, which, when vibrated ultrasonically at a frequency of $200 \mathrm{kHz}$, generate a stream of bubbles that travel to the layer deposited on an opposing glass slide. Depending on the pressure amplitude, the bubble clouds ejected from the micropits attain different shapes as a result of complex bubble interaction forces, leading to distinct shapes of the cleaned areas. We have determined the removal rates for several inorganic and organic materials and obtained an improved efficiency in cleaning when compared to conventional cleaning equipment. We also provide values of the force the bubbles are able to exert on an AFM tip.
\end{abstract}

*Published as: David Fernandez Rivas, Bram Verhaagen, James R.T. Seddon, Aaldert G. Zijlstra, Lei-M. Jiang, Luc W.M. van der Sluis, Michel Versluis, Detlef Lohse and Han J.G.E. Gardeniers. "Localized removal of layers of metal, polymer, or biomaterial by cavitating microbubbles", Biomicrofluidics (6) 034114, 2012. 


\subsection{Introduction}

Bubbles have been under analysis at least since the Greek times [1], and play an important role in physics, chemistry, medicine, and technology. The generation of bubbles by acoustic cavitation has been studied for many years, however without special measures there is little control over its occurrence and their behavior can at first sight be surprising and puzzling [2]. Whether or not cavitation due to pressure oscillations will occur, depends not only on the acoustic field, but also on physical properties and conditions such as the amount of gas in the liquid and the presence of nucleation sites. Once these are controlled, one can achieve fully controllable cavitation, in excellent agreement with the theoretical predictions [3].

Cavitation has found use in several applications, based either on its chemical or mechanical effects [4-7]. In water solutions exposed to ultrasound, for example, an enhancement of chemical reactions can be achieved. Short lived radicals form at the breakdown of water molecules, due to the high pressures and temperatures in the collapsing bubble's interior [8-10].

The use of acoustics in microfluidics and specially in bio-applications has received considerable attention in the last decade [11-13]. Acoustic cavitation can produce surface modifications, including damage, through high-speed liquids jets and shockwaves generated upon the asymmetric collapse of cavitation bubbles near a surface [14-16]. These mechanical effects have been considered by many as negative, as it may give rise to significant erosion and abrasion, for example demonstrated by the cavitation damage on ship propellers [2] and artificial heart valves [17, 18]. However, in specific cases the mechanical effect of cavitation has been shown to be useful for practical applications, for example for cell disruption and drug uptake [19, 20], or mixing and particle size reduction [21], besides several other biological effects [15, 22]. Also in electronics, cavitation has been useful for the modification of surfaces (roughening, cleaning and gold plating, among others), using liquids as benign as water. The undesired use of hazardous substances, high temperature and long process times can be avoided or reduced by the use of cavitation [23]. The use of ultrasound has therefore been considered as a "green solution" for the above mentioned applications, given the reduction in power usage and chemicals.

Nevertheless, practical uses of cavitation are in general limited because of a lack of control over the location and amount of cavitation. Under the irradiation of ultrasound to a liquid, bubbles originate from nuclei at random locations. Quantification of cavitation effects (erosion and cleaning particularly) is very difficult due to its random occurrence and also due to different materials and equipment used in different laboratories and industrial equipment [4, 24]. This situation has prevented the upscaling and extended usage of desirable effects of cavitation such as localized cleaning or surface modifications [25]. Control over cavitation location requires special measures as, for example, the use of a laser [19], by seeding the liquid with particles 
and irradiating with a shockwave [26], or from gas-filled crevices in smooth surfaces exposed to shockwaves [27], or continuous ultrasonic irradiation [28].

Here we show the generation of cavitation in various liquids for fast removal (order of tenths of seconds) of several deposited materials from a glass substrate with millimetric control, based on well-defined cavitation nucleation sites [28].

\subsection{Experimental setup}

\subsubsection{Materials to be removed}

The cleaning of surfaces is a process that is required in a wide variety of applications, with an equally wide range of materials that need to be removed from the given surface. Each of these materials and surfaces have their specific mechanical and chemical properties that provide different adhesion strengths between both. Here we list some typical materials to be removed, which are all studied in this work.

1. Solid hard materials: The solid hard materials used in this study are Gold (Au), Platinum (Pt), Chro- mium (Cr) and Titanium (Ti), all categorized as metals, and Olin and AZ96, which can be categorized as organic materials, and are commonly used as photoresists in microfabrication of semiconductors and microelectromechanical systems (MEMS).

2. Soft biomaterials: Contrary to the solid hard materials, soft biological materials may deform (visco)elastically before cohesive or adhesive failure takes place [29, 30]. Elastic materials typically display significant strain when subjected to a stress, and relax back to the initial shape and position when the stress is removed. Only when the stress exceeds the material's yield stress and enters the plastic domain of the soft material, cohesive failure will take place. Viscoelastic materials, however, exhibit viscous behavior when the stress exceeds the yield stress; the material will flow away and there will be a net displacement when the stress is removed [31, 32]. One of the most commonly encountered viscoelastic materials is the biofilm, which can often be found in pipes, on medical implants and inside and on teeth, where it often has a negative influence on efficiency or health [30, 33]. The biofilm is a structure of bacteria enclosed by a self-produced extracellular matrix [34, 35]. This matrix provides the viscoelastic properties of the biofilm, which protects the embedded bacteria from high stresses.

3. Cells: Another biological material of great importance nowadays is a cancer cell. The study of the reaction of a cell to different environmental conditions with minimal interference in their habitat is still a problem. Cavitation has 
been used to address this issue [22, 36], while it also is able to porate cells for drug uptake due to shear forces [19].

\subsubsection{Material removal experiments}

\section{Ultrasonic cavitation setup}

The continuous and localized generation of acoustic cavitation bubbles has been accomplished by ultrasonically vibrating a silicon surface containing micropits [28]. When pouring liquid over the silicon substrate, individual gas bubbles were entrapped in the pits; vibrating the surface ultrasonically resulted not only in acoustic streaming from the oscillating surface, but for large enough pressures also in pinch-off of gas bubbles from the micropits, as detailed elsewhere [37].

The silicon substrate with micropits was placed in a cavitation cell container made of glass with $25 \mathrm{~mm}$ outer diameter, $15 \mathrm{~mm}$ inner diameter and $6 \mathrm{~mm}$ depth, matching one quarter of the wavelength at the operation frequency of $200 \pm 5 \mathrm{kHz}$. A piezo element (PZ27, Ferroperm) of thickness $6 \mathrm{~mm}$ and diameter $25 \mathrm{~mm}$ was glued to the cell bottom. A sketch of the cavitation cell is given in Figure 7.1. For each experiment, the cell was filled with fresh ultra-pure water (milliQ, milliPore). Also, ethanol, bleach and acetone were investigated given their extensive use in general and ultrasonic cleaning applications. Additionally, a cell cultivation liquid medium was used.

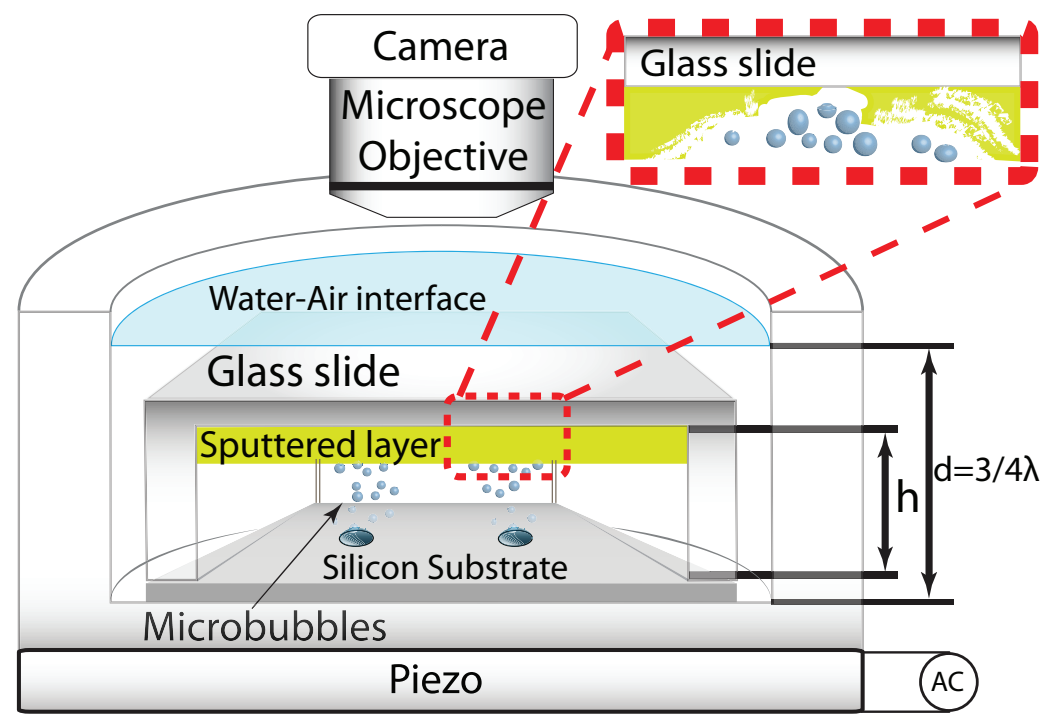

Figure 7.1: Schematic representation of the experimental setup. The gap between the glass slide and the silicon substrate $h=100 \mu \mathrm{m}$. The water column height is $5 \mathrm{~mm}$. 
The micropits were etched under clean room conditions on double-side polished silicon wafers by means of a plasma dry-etching machine (Adixen AMS 100 SE, Alcatel). The wafer was then diced in square chips with a side of $10 \mathrm{~mm}$. The edge of these substrates have a step of $58 \mu \mathrm{m}$ with four notches radially and equally spaced (see Figure 7.2).

Each pit had a diameter of $30 \mu \mathrm{m}$ and a depth of approximately $10 \mu \mathrm{m}$; multiple pits were arranged in four different configurations: single pits, two pits separated by a distance of $1 \mathrm{~mm}$, three pits arranged at the corners of an equilateral triangle with a side of $1 \mathrm{~mm}$, and four pits at the corners of a $1 \mathrm{~mm}$ side square.

The piezo element was driven by a sinusoidal signal generated by an arbitrary waveform generator (33120A, Hewlett Packard) and amplified by means of an audio amplifier (TA-FB740R QS, Sony). The voltage, current and power to the piezo element were monitored with a power meter (HM8115-2, Hameg). The duration of the experiments was short enough (typically less than 5 minutes) so that the temperature increased no more than approximately $5^{\circ} \mathrm{C}$ (measured with a T-type thermocouple with digital read-out; HI 93552R, HANNA Instruments).

Two different power settings were used with all pit arrangements: Low (0.182 W) and High $(0.629 \mathrm{~W})$. The corresponding approximate acoustic pressure amplitudes were measured to be $225 \mathrm{kPa}$ and $350 \mathrm{kPa}$, with error values provided by the manufacturer as $20 \%$. These pressures were measured using a needle hydrophone (HNR-500, Onda) positioned $65 \mu \mathrm{m}$ above the center of the chip [38].

\section{Imaging setup}

The occurrence of cavitation and the removal of the deposited materials was imaged through the air-water interface with a CCD camera (LM165M, Lumenera Corp., Ottawa, ON, Canada), recording at a frame rate of $15 \mathrm{fps}$ and an exposure time of 10 $\mu \mathrm{s}$. For the removal of metal layers, a color high-speed camera (SA2, Photron, San Diego, CA, USA) was used, recording at frame rates between 60 and 2000 frames per second and an exposure time of $30 \mu \mathrm{s}$. The cameras were attached to a microscope (BX-FM, Olympus, Tokyo, Japan) with 10x magnification, with a focal depth of approximately $10 \mu \mathrm{m}$ [39]. Illumination was provided through the microscope in dark-field mode by a continuous cold-light source (ILP-1, Olympus).

The recordings of surface layer removal were analyzed using MatLab (The Mathworks, Natick, MA, USA), by converting each frame to black and white and determining the cleaned area. The size of each pixel was determined using a calibration grid with markers of well-defined size. 


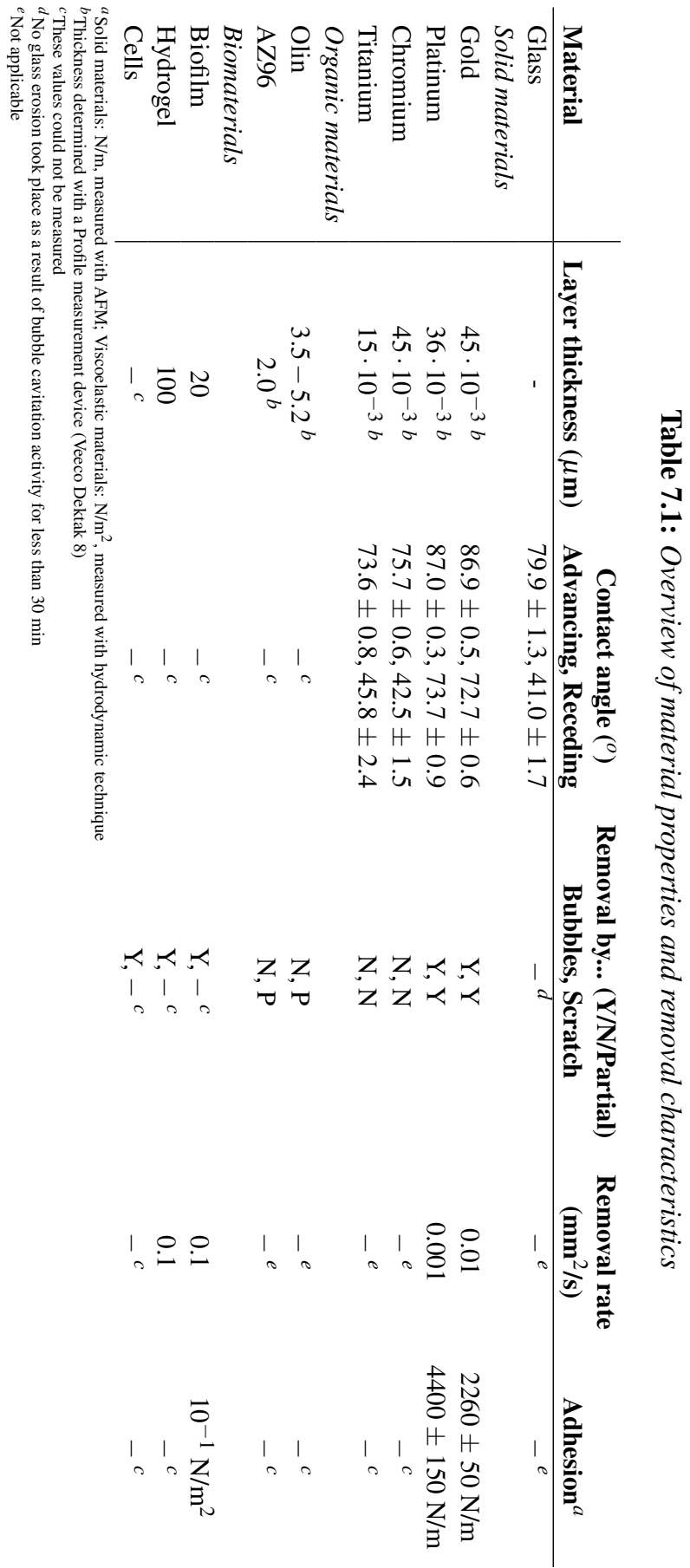




\section{Layer deposition}

The glass slides on which materials of interest were deposited were made of fused silica wafers. A circular chamber of $8 \mathrm{~mm}$ diameter and a depth of $158 \mu \mathrm{m}$ and four protruding notches that match the notches in the silicon substrate (described before) were etched on one face of each glass slide section. The wafer was then diced in chips with a side of $10 \mathrm{~mm}$. In this way when placing the glass slide on top of the silicon substrate a microchamber of $100 \mu \mathrm{m}$ height was formed (see Figure 7.2). Each material type that was investigated required a different deposition procedure, which is now outlined.

Solid hard materials A single wafer sputter coater (home-made system available in MESA+ Nanolab) for deposition of metallic layers was used to deposit gold, platinum, chromium and titanium. To deposit organic materials (photoresists Olin 35 and AZ96), hexamethyldisilazane (HMDS) was first spin-coated as a primer to provide chemical bond of the photoresists and strong adhesion to the glass slide on a single wafer spin-coater (Primus coater WB21).

Biomaterials The biofilm model used here was described recently for use in endodontics [40]. In brief, round glass coverslips attached to a customized stainless steel lid were suspended in a medium inoculated with bacteria. In this model, biofilm attachment and growth is an active process against gravity. Microbiology Cells of E. faecalis V583 and P. aeruginosa HG 1776, both clinical isolates, were grown and maintained as pure cultures on blood agar plates. E. faecalis was cultured under anaerobic conditions $\left(80 \% \mathrm{~N}_{2}, 10 \% \mathrm{H}_{2}\right.$ and $\left.10 \% \mathrm{CO}_{2}\right)$ whilst $P$. aeruginosa was cultured in air, both at $37^{\circ} \mathrm{C}$. Growth medium for overnight cultures of biofilms was BHI broth containing $0.5 \mathrm{BHI}, 50 \mathrm{mmol} / \mathrm{L} \mathrm{1,4-piperazinediethanesulfonic} \mathrm{acid,}$ buffer, $5 \%$ saccharose and $1 \mathrm{mmol} / \mathrm{L} \mathrm{Ca}^{2+}$. Phosphate-buffered saline was used to wash during refreshment of the medium. E. faecalis and P. aeruginosa were inoculated in wells with additional $\mathrm{Ca}^{2+}$ in the broth of a 24-well multiwell plate. Round glass coverslips, diameter $12 \mathrm{~mm}$ and thickness $140 \mu \mathrm{m}$, mounted on a custom-made stainless steel lid, were suspended in the broth. In this experiment, biofilms grown for 96 hours were used. The broth was refreshed daily. Inoculation of bacteria and the biofilm growth took place at $37^{\circ} \mathrm{C}$ in air. The thickness of the biofilm was estimated to be $20 \mu \mathrm{m}$, with some bacteria colonies protruding up to $50 \mu \mathrm{m}$ above the glass substrate.

A biofilm was mimicked with a hydrogel, created by dissolving $1 \mathrm{~g}$ gelatin (Merck, Whitehouse Station, NJ, USA), 0.02 g hyaluronan (Sodium hyaluronate 95\%, Fisher, Waltham, MA, USA) and $0.1 \mathrm{~g}$ red food color (KTC, Wednesbury, UK) in $45 \mathrm{~mL}$ warm water[41]. Hollow glass spheres with a mean diameter of $10 \mu \mathrm{m}$ (Sphericel, Potters Industries, South Yorkshire, UK) were added as tracer particles. The final density of the hydrogel was $1.07( \pm 0.07) \cdot 10^{3} \mathrm{~kg} / \mathrm{m}^{3}$. This hydrogel was cooled down to room temperature before depositing on a glass slide with raised edges of 
approximately $100 \mu \mathrm{m}$ height. The glass slide was then left to cool down for 7 or 15 minutes, during which the hydrogel dries and attaches to the glass, before the ultrasound and imaging was applied.

MCF-7 human breast carcinoma cells were cultured on the same glass slides; its adhesion resistance when exposed to the cavitation bubbles from the triangular array of micropits was investigated in the cavitation setup. The culture medium was made of Dulbecco's Modified Eagle Medium (DMEM, Gibco), L-Glutatmate, fetal bovine serum (FBS), Antibiotics (Penicillin and Stretopmycin) and Fungicide.

Table 7.1 gives an overview of the various deposited materials and their properties.

\subsubsection{Cavitation damage}

The glass substrate onto which the gold was deposited, was inspected for erosion damage using a scanning electron microscope (SEM, HR-SEM 1550, Zeiss). After the gold was removed by cavitation, the glass substrates were exposed to cavitation for another 1, 5, 10, 15 or 30 minutes before SEM images were taken.

\subsubsection{AFM up-thrust measurements of cavitation cloud force}

An Atomic Force Microscope (AFM, Agilent 5100) in non-contact tapping mode was used to measure the up-thrust force from the bubble cloud. A silicon $n^{+}$flat disc tip cantilever (Nanosensors PL2-NCLR-10, spring constant of $48 \mathrm{~N} / \mathrm{m}$ and resonant frequency $190 \mathrm{kHz}$ in air and $73 \mathrm{kHz}$ in water) with a diameter of ca. $1.8 \mu \mathrm{m}$ and tip height of ca. $12 \mu \mathrm{m}$ was used. The cantilever was withdrawn $100 \mu \mathrm{m}$ from a single-pit substrate surface to mimic the separation between the micropits and 'tobe-cleaned' surfaces in the previous measurements. Both low and high ultrasonic powers were used, although for the high power the resulting signal was clipping. The cantilever approached from a distance of $1 \mathrm{~mm}$ in a line passing over the bubble clouds. As it happens in the experiments with the glass slides and deposited layers, the bubbles traveled to the cantilever and collapsed against it. Unfortunately, the cantilever was destroyed during the second pass at this force, so the measurements were stopped.

\subsection{Results}

\subsubsection{Cavitation occurrence, in the absence of deposited materials}

Switching on the ultrasound leads to a continuous generation of microbubbles with a size range of 2-17 $\mu \mathrm{m}$, determined from the video recordings and shown in Figure 7.8 (see also Fernandez et al. (2012) [38]). Bubbles smaller than $2 \mu \mathrm{m}$ could not 
be visualized due to the resolution of the camera. Varying the pressure amplitude (power) and the different arrangements of pits resulted in different bubble patterns, as a result of the complex interplay of primary and secondary Bjerknes forces, microstreaming and also the inertial forces acting on the moving microbubbles in the close vicinity of the glass slide. The bubbles were observed to travel towards the glass slide on top of them where they come in focus of the optical setup. This upward motion is a result of the effect of secondary Bjerkness forces, which makes bubbles close to a hard surface "feel" the presence of their image bubble as an attraction force, driving the bubbles closer to the wall.

Increasing the power resulted in an increase in the number of ejected bubbles around the pits, forming a bubble cloud or cluster. At high power, with more than one pit, the bubble clusters are attracted to each other and at a given power level (between 0.182 and $0.629 \mathrm{~W}$ ), the bubbles start to travel towards the geometrical center of the pit array [28, 38] (see Figure 7.7 left column). These bubble cluster configurations are reversible, although there is hysteresis in the amplitude necessary to return to the separated clouds, which is lower than needed for making the bubbles travel to the geometrical center of the pit configuration.

\subsubsection{Removal of deposited materials by cavitation}

\section{Observations}

Compared to the experiments with and without glass slide, a clear difference can be observed in terms of stabilizing the free water-air interface oscillation when the glass slide is not present. When the glass slide is placed, the traveling and standing wave components change dramatically, contributing to complex effects of bubble cavitation conditions (measured for example through the occurrence of sonochemical reactions) [42].

The opaque materials deposited on the glass slide, when placed on top of the cavitation cell, did not allow for optical visualization of the cavitation bubbles. However, taking as a representative example the case of deposited gold, when switching on the ultrasound at low power $(0.182 \mathrm{~W})$, we see that almost instantly (within 2 seconds) a small opening appears in the gold layer right on top of where the pits are located (Movie 1). If the power is kept constant the removed gold layer area increases slowly over time. For the case of one pit, increasing the power makes the removed area increase until a maximum area is reached (approximately $0.03 \mathrm{~mm}^{2}$ ). Increasing the power for the two, three and four pit geometries, for which situation the bubbles travel to the geometrical center as described above, an area corresponding to the area of the bubble trajectories is removed. Therefore, for two pits a linearly extended area is typically observed. Accordingly, we find a triangular shape for the three pits and a distorted rectangular shape for the case of four pits; see Figure 7.2 and Figure 7.7. 
right column. For Au and $\mathrm{Pt}$ we observed a similar surface removal behavior.

For the cases of deposited $\mathrm{Cr}$, Ti and the organic materials, there was no surface removal with the same initial conditions as for Au and Pt. Manually creating a scratch in the layer close to the geometrical center allowed for confirming the presence of the bubbles in the scratched areas, but no material was removed from $\mathrm{Cr}$ and $\mathrm{Ti}$ layers. After scratching of the photoresist materials the bubbles were able to detach a few pieces of photoresist material only occasionally (Figure 7.3 and Movie 2). The removed pieces of (all) layers could sometimes be trapped by the oscillating bubbles for a relative long period of time (at least more than $30 \mathrm{~s}$ if no conditions changed).

When the ultrasound is switched off abruptly, the pits may be expected to be filled with water and perhaps, if a bubble is still inside any of the pits, are unable to nucleate more bubbles, deactivating the acoustic bubble generation. If ultrasound is turned on again therefore no cavitation from the micropits takes place. This is why this procedure allows for studying the effect of acoustic streaming alone on material removal. We could not observe removal of any material in the situations where no bubbles were present, allowing us to conclude that the forces associated to streaming alone were not sufficient to remove any of the tested materials. The quantification of streaming from the air-filled pits could not be assessed with the current setup.

The biofilm that was grown on a glass substrate was initially visible as a gray area containing dark clusters of bacteria (Figure 7.4 and Movie 3). Due to transparency of the biofilm the bubble cloud below the biofilm-covered substrate was visible when the ultrasound was turned on. The biofilm was then removed quickly when the bubble cloud was attracted by the biofilm-covered substrate. No biofilm was removed by the streaming or by bubble clusters when not attracted to the glass surface. A similar behavior was observed for the hydrogel. The hydrogel appeared softer and attached with weaker force than the biofilm, both after 7 and 15 minutes of drying, but neither was removed by the acoustic streaming alone.

The distance between the hydrogel or biofilm and the micropits was less welldefined than for the metal and hard organic deposited layers, due to the non-uniform thickness of these layers and flexible (softer) nature. Therefore the bubbles were less likely to travel toward these layers, which resulted in slow or no increase in removed area after initial removal.

The MCF-7 cells grown on the glass substrate were also removed when the bubbles were present, leaving the outlier cells attached, as can be seen in Figure 7.5. The liquid cultivation media produced bubbles similar to those in water. The contour of the removed area resembles again the shape of the bubble cloud for the three pits configuration. 
a)

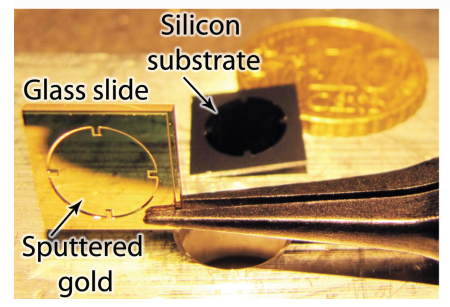

b) Removed material

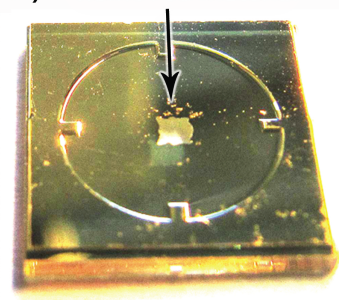

Figure 7.2: a) A black tweezer holds a gold sputtered glass slide prior to exposure to cavitating bubbles; in the background a silicon substrate is shown. b) A zoomed view of the same glass slide after exposure to cavitating bubbles from four pits in the cavitation cell described in this paper. The glass slide has sides of $10 \mathrm{~mm}$.

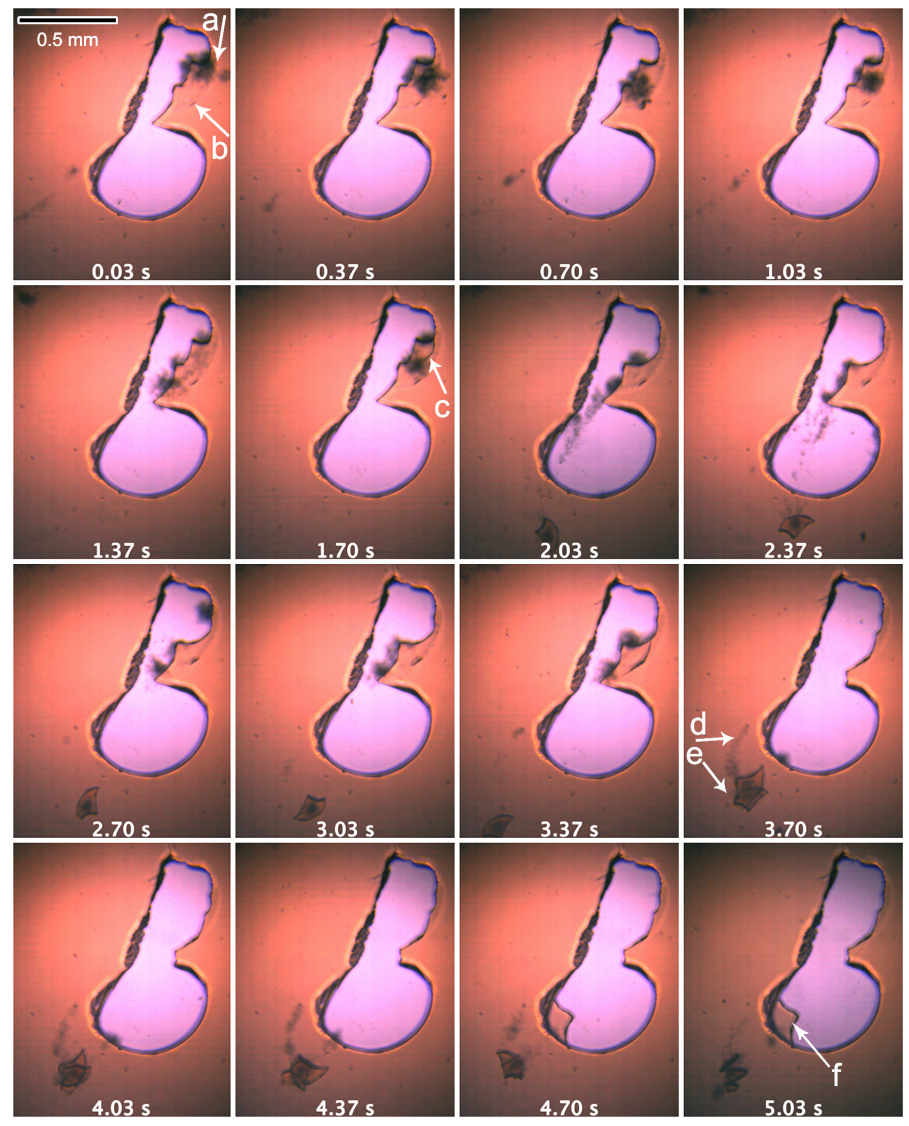

Figure 7.3: Montage of a time series of frames recorded for the case of AZ96 photoresist. The center part of each frame shows a portion of manually removed AZ96. Arrow a points out a cloud of bubbles as a darker region cavitating close to a crack in the AZ96 layer (arrow b). Arrow c shows how the crack grew within about 1 second. After 1.7 s, the bubbles (arrow d) "trapped" a small portion of AZ96 as indicated by arrow e. Eventually, after approximately $4.7 \mathrm{~s}$, the small portion gets stuck in the border line of removed material. The pits do not appear in these pictures since they are out of the field of view (Movie 2). 


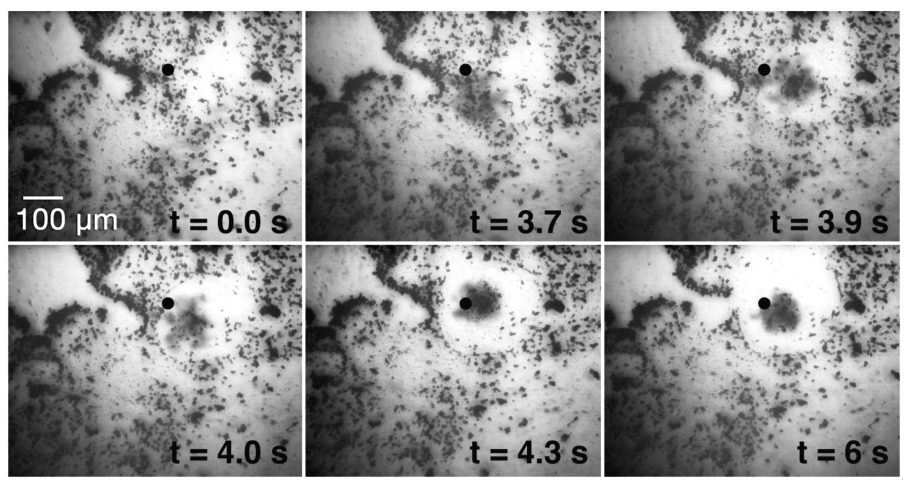

Figure 7.4: Stills from a recording of a biofilm being removed by bubbles. The gray area with black dots is the area covered by biofilm, the location of the pit is indicated with a large black dot. The bubbles can be identified as the blurred dark region around the pit (Movie 3).

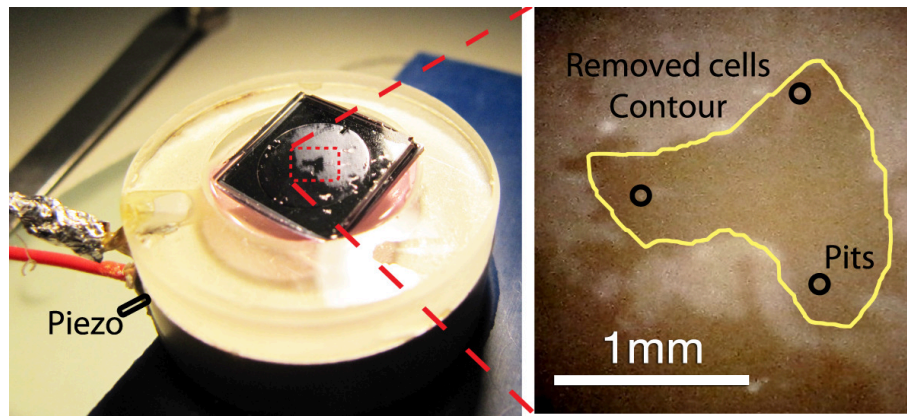

Figure 7.5: Cavitation cell and experimental results after removing MCF-7 cells fixed to a glass slide. The liquid cultivation media's pinkish color is visible inside the cavitation cell glued to the piezo.

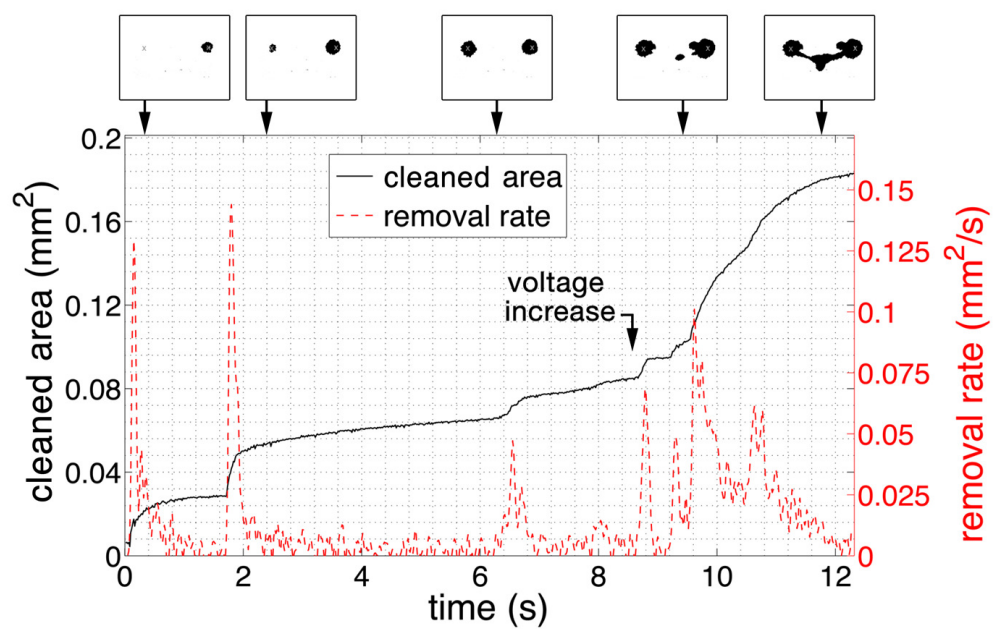

Figure 7.6: Size of cleaned area and its derivative (i.e., removal rate) extracted from video measurements 


\section{Removal rates}

The area in which gold has been removed by the bubbles originated from a single pit, after approximately 1 minute, is of the order of $0.1 \mathrm{~mm}^{2}$; for 4 pits this area increases by one order of magnitude to $1 \mathrm{~mm}^{2}$, see figure 7.6 . The rate of removal during the initial growth of the removed area is of the order of $0.1 \mathrm{~mm}^{2} / \mathrm{s}$; this reduces by one order of magnitude to $0.01 \mathrm{~mm}^{2} / \mathrm{s}$ during steady growth. For platinum, once a scratch had been made, the steady removal rate is of the order of $0.001 \mathrm{~mm}^{2} / \mathrm{s}$.
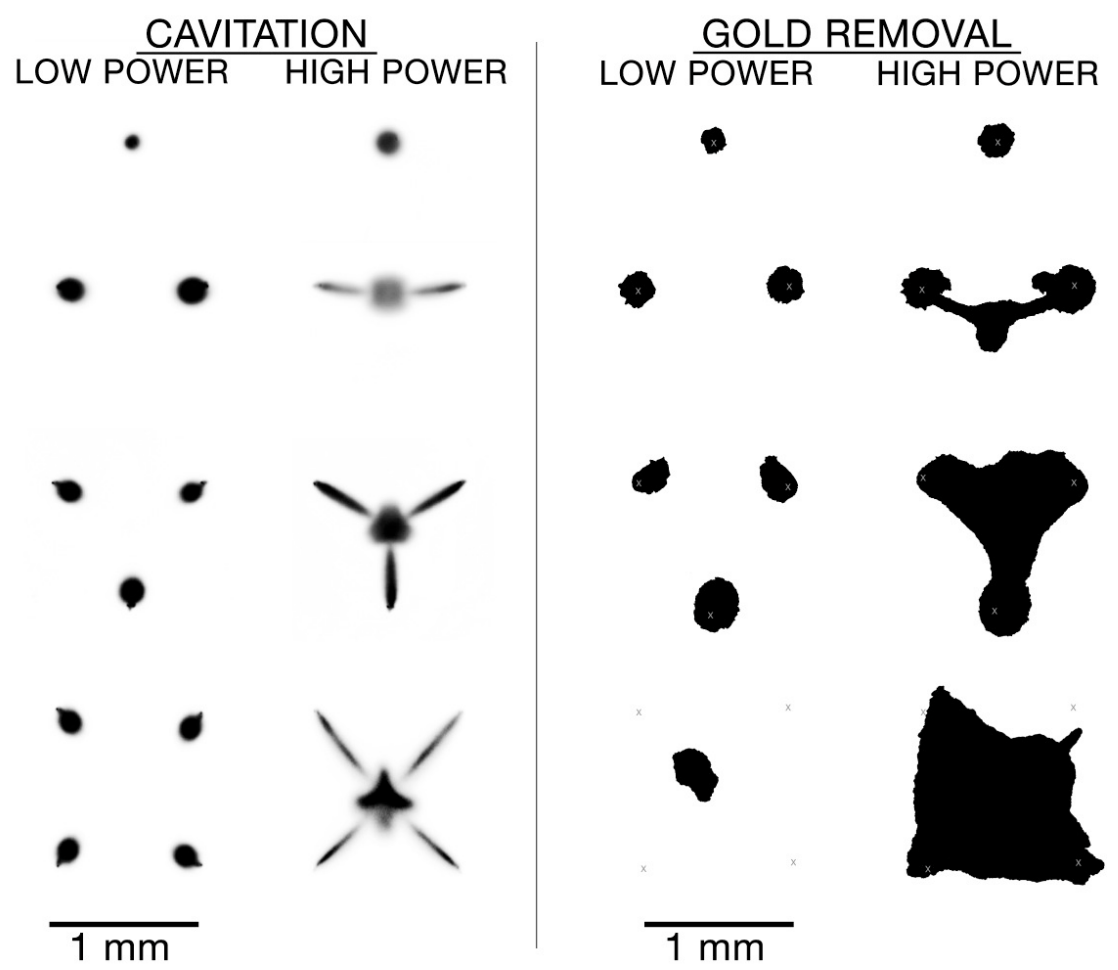

Figure 7.7: From top to bottom and left to right, the cases for 1 to 4 pits at low and high power. Left: Bright field illumination (inverted color) showing bubble patterns with slow speed imaging. Right: Contour shape of cleaned area in gold (black) due to bubble cavitation.

The removal of the hydrogel and the biofilm were recorded at lower imaging speeds, providing less detailed information on the removal rates. However, estimates for the removal rate of the hydrogel give a value similar to that of gold, being of the order of $0.01 \mathrm{~mm}^{2} / \mathrm{s}$, with initial removal rates up to $0.1 \mathrm{~mm}^{2} / \mathrm{s}$. The initial rate of removal of the biofilm was estimated to be $0.1 \mathrm{~mm}^{2} / \mathrm{s}$ as well, with only minor increase in removed area after the initial removal.

The cleaning effect of bubbles is restricted locally by how close the pits and the 
nucleated bubbles are from the surface to be cleaned. The bubbles cannot travel farther than a given distance away from the pits, or nearby other bubbles. This is due to the required conditions for continuous cavitation such as available gas content and negative pressure values provided by the oscillating pressure field.

\subsubsection{Different liquids}

Bubbles were observed to be generated in all five liquids investigated. Individual images are shown in Figure 7.8 . For water and bleach the bubble shapes are similar, with an apparent higher number of bubbles than for ethanol and acetone. For these two organic liquids we can see smaller bubbles ejected from the pits and a larger bubble growing at the midpoint that, upon reaching a given size, floats away. The experiments with MCF-7 cells were carried out with the same liquid cultivation media showing similar bubble cavitation as in water (see Figure 7.5).

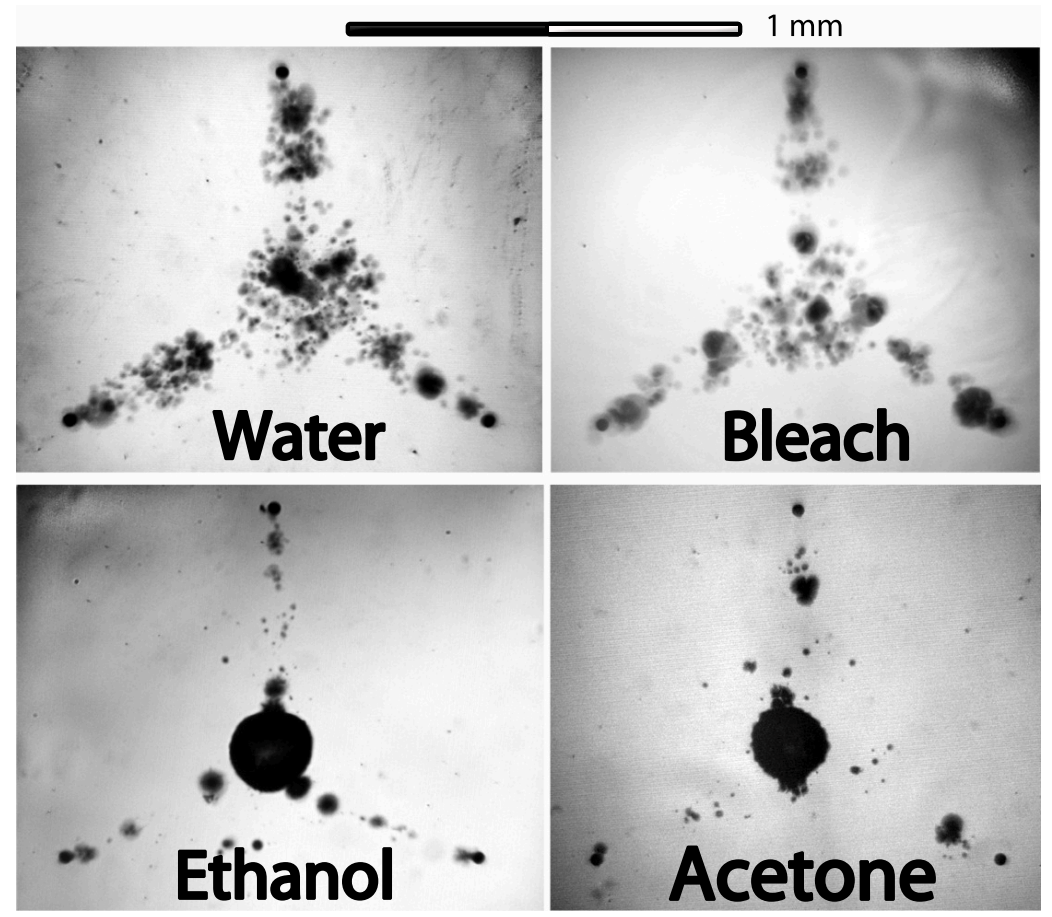

Figure 7.8: Bubble pattern for four different liquids tested (no glass slide on top). The bubbles generated are clearly different for each substance but qualitatively similar between water and bleach and between ethanol and acetone.

We expect similar removal effects for these liquids as for water, but it was not within the scope of this paper to address this topic further. 


\subsubsection{Cavitation erosion}

After the maximum period of 30 minutes of ultrasound exposure, no erosion was visible on the glass surface, as investigated by SEM analysis. This shows a different effect than the erosion observed on Si surfaces in our previous studies [38]. The reasons for this are that $\mathrm{Si}$ has a crystalline atomic structure and is brittle whereas glass is amorphous and does not exhibit the same mechanical properties (see Chapter 87. Additionally, the erosion we observed on $\mathrm{Si}$ surfaces was on the substrates from which the bubbles were generated, whereas here the bubbles have travelled a considerable distance ( $150 \mu \mathrm{m}$ from its nucleation sites) to the glass surface, and the acoustic conditions have changed considerably.

\subsubsection{Bubble cloud forces}

The AFM measurements showed that the force was intermittent, with an average deflection of the cantilever of ca. $500 \mathrm{~nm}$, which corresponds to a force of $20 \mu \mathrm{N}$. Using the tip area of the cantilever, the pressure exerted by the bubble cloud can be estimated to be of the order of $50 \mathrm{~atm}$.

The force measurements in both directions of sweep were symmetric, which is especially important for the direction that runs along the length of the cantilever, across the tip, and into free water (where there are no bubbles present). The sweep force had a plateau maximum attributed to the cantilever being directly above the pit, plus slightly to either side but with the bubble streams redirecting to the tip. The value of the force given is the average of the maxima of both the lateral and long ways sweeps, which agree with each other to within error, which thus represents the bubbles hitting the end of the cantilever.

The sweeps pass over the end of the cantilever so we assume that the force measurements can be interpreted in the usual sense in AFM measurements. That the sweep along the length of the cantilever and into free space appears symmetric, represents the fact that the cantilever's "lever" is more than double the distance away from the surface.

Finally, in terms of incorrectly interpreting interference from bubbles as deflection, the laser spot is wholly and exclusively on the back side of the lever (we do not see interference with the bare substrate from an overlapping spot, as an example of the standard control measure). The chip is much wider than the probe's $2 \mu \mathrm{m}$ end and all bubbles direct towards the end and terminate there for the cases when we use the force measurement.

Also, the temporal resolution limit is given by the resonant frequency $(190 \mathrm{kHz}$ in air and $73 \mathrm{kHz}$ in water), which is lower than the piezo frequency $(200 \mathrm{kHz})$, so we measure only averages of impacts and not individual events. 


\subsection{Discussion}

\subsubsection{Cleaning action of bubbles}

With the micropits employed in this study it was shown that metal thin layers (Au and Pt), biomaterials (biofilm, cells and hydrogel) and organic materials (photoresists) could be removed in a certain local area, defined by the bubble cloud from the micropits. Surprisingly, the removal rates were similar for the metal and the viscoelastic layers, while they all have entirely different mechanical and adhesive properties. This suggests that the bubbles can exert enough mechanical force to remove all of these materials, but that the amount of material that each bubble can remove during one cycle is limited. The deposited material will have to be removed piece by piece, cycle after cycle as a result of several bubbles (clusters) cavitating close to a layer (see Figure 7.5). As more material gets removed, the bubbles have to travel a larger distance from their origin (pit or geometrical center of the pits array), but are becoming less likely to do so, consequently slowing down the removal rate.

When acoustic cavitation bubbles are in contact with hydrophobic surfaces, it could be expected that no cushion layer of liquid exists that would dampen the liquid jet impact as a result of bubble collapse (the hydrophobic or -philic properties of surfaces and adhesion forces among them have been reported elsewhere) [43-45]. Accordingly, the hydrophobicity of the deposited material might have an influence on the material removal, as for bubbles it is easier to collapse towards a hydrophobic wall. It has been reported that the hydrophobic force is uniquely determined by the contact angle [46]. Interestingly, water on $\mathrm{Au}$ and on $\mathrm{Pt}$, both removed by the cavitating bubbles, has a much higher (receding) contact angle than on the other metals (see Appendix 7.D and Table 7.1 for contact angle measurement details and results), suggesting that the bubbles could reach those two metals more easily than the other metals. It is not known how much this effect contributes to the cleaning effect.

The fact that microbubbles were generated in ethanol, acetone, bleach, and cell cultivation medium as well as in water suggests that the cleaning with the help of microbubbles can be enhanced for chemical cleaning processes. AZ96 and Olin are commonly removed by acetone; bleach is used routinely in biological/medical applications like dentistry to kill bacteria. According to the results of this study, cavitation could mechanically enhance the chemical removal.

We focused on water since is the "greenest" solvent normally used in cleaning applications but other liquids may be used with similar effects. Another important fact related to our experiment is the small volumes needed to remove the desired substrates. This becomes important when considering cleaning applications with less green solvents (e.g. acetone, toluene, etc.).

To address the influence of the radicals produced by the cavitating microbubbles 
[28] on the removal of deposited metal materials, all the metals were exposed to hydrogen peroxide (Sigma-Aldrich 30\% as provided). For Au there was no reaction observed at least for the first 5 minutes, after which occasionally some small regions presented small detachment areas (ca. $1 \mathrm{~mm}^{2}$ ). For Pt there was some chemical activity observed (bubbling and gas formation, presumably $\mathrm{H}_{2} / \mathrm{O}_{2}$ ) that led to detachment of virtually the complete layer in a couple of seconds. For $\mathrm{Cr}$ and Ti, there was no reaction within the observation time of 10 minutes. This allows us to conclude that if there is any contribution of chemical radicals (e.g. $\mathrm{H}^{\cdot}$ or $\mathrm{OH}$ ) to the removal rates it will be minimal under the conditions attained with cavitation bubbles nucleated from the pits.

The acoustic microbubble generation from the micro pits can be sustained for at least several hours if the temperature is kept constant and gas loss is prevented. This means that the loss of gas associated to the microbubble generation does not deactivate the pits, evidencing a process of rectified gas diffusion into the pits. More details on the bubble behavior can be found elsewhere [28, 38].

We observed stable cavitation from the pits with the glass slide placed on top for at least $30 \mathrm{~min}$. However, normally the removed layer areas did not increase much more after the first $3 \mathrm{~min}$ if no change in the ultrasound conditions or moving of the glass slide took place. Additionally, the low operation power compared with conventional ultrasonic cleaning devices (one order of magnitude lower in our case) and the control over the areas to be removed, makes the present cleaning device useful in several applications.

\subsubsection{Detachment kinetics}

\section{Solid hard materials}

The detachment of material layers by acoustic cavitation bubbles can occur due to material defects, at which the bubbles can perform further detachment. Once a crack is formed, detachment could proceed by peeling and tearing mechanisms due to bubble jetting, and presumably also due to shock waves generated at cavitation bubble collapses and reflections from the hard surfaces. The contribution of streaming can be ruled out based on the above reported observations that there is no cleaning for streaming only (without bubbles). Note that the removal of a thin film may follow a different timeline than traditional cavitation erosion in bulk materials, as the material properties of the bulk and a layer of the same material might be different [47]. To explain the differences in cleaned area and removal rates between the various metals we have to consider their different adhesion characteristics.

For the metals, a first estimation can be obtained by considering the formation of metal oxides on the surface of the metals. Even though the films are sputtered under Argon atmosphere conditions, the thickness of all layers is small enough to allow 
metal oxides to form on the surface. It is known that thin films for oxygen active metals have a higher adhesion to glass than films of noble metals [48] because of molecular electrostatic bonds between the metal oxides and glass [49].

The likeliness of a metal to form an oxide layer can be obtained from its affinity of oxygen, which is directly related to the (negative) heat of oxide formation of the metal. $\mathrm{Au}$ and $\mathrm{Pt}$ (heats of oxide formation of $79.5 \mathrm{~kJ} / \mathrm{mol}$ and $-134 \mathrm{~kJ} / \mathrm{mol}$ for $\mathrm{Au}_{2} \mathrm{O}_{3}$ and $\mathrm{PtO}_{2}$, respectively) do not oxidize easily since these are noble metals, and are therefore expected to have a lower adhesion to glass than $\mathrm{Cr}$ and $\mathrm{Ti}$ (heats of oxide formation of $-1130 \mathrm{~kJ} / \mathrm{mol}$ and $-912.1 \mathrm{~kJ} / \mathrm{mol}$ for $\mathrm{Cr}_{2} \mathrm{O}_{3}$ and $\mathrm{TiO}_{2}$ respectively) [50, 51].

The bonding of photoresists (AZ96 and Olin) to glass is strengthened by the strong chemical bond provided by the HDMS priming step. For that reason, only where defects in the layer existed, the bubbles were able to remove material.

The exact value for the adhesion for these solids is difficult to obtain, as the ways and experimental techniques to quantify it are still controversial [52]. At the root of this problem lies the fact that the individual surfaces to be bonded (metal/inorganic layers and glass surface in our case) have different properties when bonded than when separated. Many factors can affect a bond, such as impurities, roughness, defects or inhomogeneity on any of both substances, actions of the environmental and mechanical stresses.

Therefore, instead of theoretically predicting the adhesion of the solids of which the layer and substrate are made off, it is more relevant to attempt to measure the actual deposited materials' adhesion with a given experimental system or technique. There are many adhesion measurement techniques described in the literature, including nucleation methods (based on deposition, only able to give basic adhesion values) and mechanical methods (direct pull-off, moment or topple, ultracentrifugal, ultrasonic, adhesive tape peel, scratch, abrasion, laser spallation, etc.) [47]. However, the values provided by one technique or given apparatus might not coincide with another technique or with values given in the literature for similar systems. Comparison with the literature should therefore be treated with care, and it is advisable to perform adhesion tests in each individual system [50].

In order to get a qualitative comparison for the adhesion of the solid hard materials used in this study, we have performed our own scratch tests on the solid hard materials, using an AFM and standard adhesive tape test. The details can be found in Appendices 7.A and 7.B respectively; the relative adhesion strength of these materials can be summarized as follows: $\mathrm{Au}<\mathrm{Pt}<$ Olin and $\mathrm{Az} 96<\mathrm{Ti}$ and $\mathrm{Cr}$.

As another qualitative comparison, and more relevant to ultrasonic cleaning, the removal of the solid hard materials was investigated in a commercial ultrasound bath (Appendix 7.C), showing non-localized removal of Au and Pt under maximum ultrasound conditions, or no removal after 10 minutes. Taking as an example the case 
of $\mathrm{Au}$, our technique provided better localized and faster layer removal (compare Figures 7.2 and 7.11 .

\section{Soft biomaterials}

Biofilms are known to be able to adhere strongly to a substrate. The initial state of biofilm formation is the adhesion of planktonic bacteria to a substrate, after which the biofilm structure is developed. The bacteria themselves adhere to a substrate due to a multitude of forces, based on both physicochemical and molecular/cellular interactions [53, 54]. During its mature state, the biofilm has also been found to increase its adherence through the production of functional amyloids, among others [30].

Literature reports a wide range of viscoelastic and adhesive properties of encountered biofilms, as these properties depend on the ecology of the biofilm and also on the conditions under which the biofilm has been grown [29, 30, 54-56]. Furthermore, the properties of the substrate itself are important, for example the surface roughness and hydrophobicity [30, 54].

A hydrogel can have better defined viscoelastic properties, as there is more control over its production, and can be used as a substitute for a biofilm when studying the mechanical behavior of biofilms [57]. However, a hydrogel is not grown but deposited onto the glass slide and therefore its attachment to a substrate is expected to be generally weaker than for a biofilm, relying only on physicochemical interactions between glass and hydrogel proteins [58].

The elastic modulus and adhesion of the hydrogel were estimated using a hydrodynamic technique, of which the details and results are given in Appendix 7.E. The hydrogel was observed to have a weaker attachment to the substrate than the typical values for a biofilm, which is in agreement with our handling observations. However, considering the observation that the hydrogel did show viscoelastic behavior, it can be considered a soft biofilm. The mechanical properties of the hydrogel can be further tuned by changing its composition and through cross-linking.

The viscoelastic materials were not removed by the acoustic streaming alone, whereas the cavitation cloud could remove them locally within a few seconds. An explanation for this different behavior could be the timescales at which both components react. The pressure magnitude and fluctuations associated with acoustic streaming could be still in the elastic regime of the viscoelastic behavior; the pressure associated with transient bubble dynamics, however, can locally have a high magnitude and fast fluctuation, causing deformations in the viscous regime, and possibly even in the plastic regime.

Many of the MCF-7 cells grown on the glass slide had been detached due to the induced cavitation cloud. However, optical visualization does not give any information on the cells around the area that was cleaned. Those cells might have been lysed 
through sonoporation, as has been demonstrated by Ohl et al. [59]. Their method of dead-live staining could provide the information on the viability of the remaining cells.

\subsection{Conclusions}

The localized generation of cavitation from micropits was shown to be able to remove deposited thin metal and organic films and biomaterials on a millimetric scale in less than 1 minute. The shape and extent of the cleaned area is related to the number of micropits; the rate of removal was of the order of $0.1 \mathrm{~mm}^{2} / \mathrm{s}$. Basic adhesion tests provided some insight into the likeliness of a material to be removed.

The low operation power and small volumes of liquid required, together with the limited cavitation erosion damage, make the cleaning device presented here a "green" solution for localized cleaning in many applications. Additionally, the possibility to create patterned surfaces on a thin film deposited on a glass substrate would be highly appreciated in fields such as Optoelectronics.

\section{Acknowledgments}

The authors acknowledge Stefan Schlautmann from the MCS group, University of Twente for his support in the microfabrication processes, Roy H.A. Visser from the CTW group, University of Twente for lending surface adhesion equipment and R. de Ruijter from the PCF group, University of Twente, for assistance in the contact angle measurements. The cells were provided and cultured by A. Sridhar and Prof. S. le Gac from the BIOS group, University of Twente.

\section{References}

[1] A. Prosperetti, "Bubbles", Phys. Fluids 16, 1852-1865 (2004).

[2] D. Lohse, "Bubble puzzles", Phys. Today 56, 36-41 (2003).

[3] B. M. Borkent, S. Gekle, A. Prosperetti, and D. Lohse, "Nucleation threshold and deactivation mechanisms of nanoscopic cavitation nuclei", Phys. Fluids 21, 102003 (2009).

[4] S. Barnett, G. Ter Haar, M. Ziskin, W. Nyborg, K. Maeda, and J. Bang, "Current status of research on biophysical effects of ultrasound", Ultrasound Med. Biol. 20, 205-218 (1994).

[5] M. Ashokkumar and T. J. Mason, Sonochemistry (John Wiley \& Sons, Inc.) (2000).

[6] J. H. Bang and K. S. Suslick, "Applications of ultrasound to the synthesis of nanostructured materials", Adv. Mater. 22, 1039-1059 (2010).

[7] G. Cravotto and P. Cintas, "Harnessing mechanochemical effects with ultrasound-induced reactions", Chem. Sci. 3, 295-307 (2012). 
[8] M. P. Brenner, S. Hilgenfeldt, and D. Lohse, "Single-bubble sonoluminescence”, Rev. Mod. Phys. 74, 425-484 (2002).

[9] Y. T. Didenko and K. S. Suslick, "The energy efficiency of formation of photons, radicals and ions during single-bubble cavitation”, Nature 418, 394-397 (2002).

[10] D. Lohse, “Sonoluminescence-Cavitation hots up", Nature 434, 33-34 (2005).

[11] L. Y. Yeo and J. R. Friend, "Ultrafast microfluidics using surface acoustic waves", Biomicrofluidics 3, 012002 (2009).

[12] P. Marmottant and S. Hilgenfeldt, "Controlled vesicle deformation and lysis by single oscillating bubbles", Nature 423, 153-156 (2003).

[13] R. Karshafian, P. D. Bevan, R. Williams, S. Samac, and P. N. Burns, "Sonoporation by ultrasound-activated microbubble contrast agents: Effect of acoustic exposure parameters on cell membrane permeability and cell viability", Ultrasound Med. Biol. 35, 847 - 860 (2009).

[14] S. Ohl, E. Klaseboer, and B. Khoo, "The dynamics of a non-equilibrium bubble near bio-materials", Phys. Med. Biol. 54, 6313-6336 (2009).

[15] C.-D. Ohl, M. Arora, R. Ikink, N. De Jong, M. Versluis, M. Delius, and D. Lohse, "Sonoporation from jetting cavitation bubbles", Biophys. J. 91, 4285-4295 (2006).

[16] C.-D. Ohl, T. Kurz, R. Geisler, O. Lindau, and W. Lauterborn, "Bubble dynamics, shock waves and sonoluminescence", Philos. T. Roy. Soc. A 357, 269-294 (1999).

[17] H. Lee, A. Homma, E. Tatsumi, and Y. Taenaka, "Observation of cavitation pits on mechanical heart valve surfaces in an artificial heart used in in vitro testing”, J. Artif. Organs 13, 17-23 (2010).

[18] E.-A. Brujan, “Cardiovascular cavitation”, Med. Eng. Phys. 31, 742-751 (2009).

[19] R. Dijkink, S. Le Gac, E. Nijhuis, A. Van den Berg, A. Vermes, A. Poot, and C.-D. Ohl, "Controlled cavitationcell interaction: trans-membrane transport and viability studies”, Phys. Med. Biol. 53, 375 (2008).

[20] C.-D. Ohl and B. Wolfrum, "Detachment and sonoporation of adherent hela-cells by shock wave-induced cavitation", Biochim. Biophys. Acta 1624, 131-138 (2003).

[21] T. Mason, A. Cobley, J. Graves, and D. Morgan, "New evidence for the inverse dependence of mechanical and chemical effects on the frequency of ultrasound", Ultrason. Sonochem. 18, 226 - 230 (2011).

[22] P. Riesz and T. Kondo, "Free radical formation induced by ultrasound and its biological implications", Free Radical Biol. Med. 13, 247-270 (1992).

[23] A. Cobley, L. Edgar, M. Goosey, R. Kellner, and T. Mason, "Initial studies into the use of ultrasound to reduce process temperatures and chemical usage in the pcb desmear process", Circuit World 37, 15-23 (2011).

[24] T. Okada, Y. Iwai, S. Hattori, and N. Tanimura, "Relation between impact load and the damage produced by cavitation bubble collapse", Wear 184, 231-239 (1995).

[25] P. Gogate and A. Pandit, "Sonochemical reactors: Scale up aspects", Ultrason. Sonochem. 11, 105-117 (2004).

[26] M. Arora, C. Ohl, and D. Lohse, "Effect of nuclei concentration on cavitation cluster dynamics", J. Acoust. Soc. Am. 121, 3432-3436 (2007).

[27] N. Bremond, M. Arora, C.-D. Ohl, and D. Lohse, "Controlled multibubble surface cavitation", Phys. Rev. Lett. 96, 224501 (2006). 
[28] D. Fernandez Rivas, A. Prosperetti, A. G. Zijlstra, D. Lohse, and H. J. G. E. Gardeniers, "Efficient sonochemistry through microbubbles generated with micromachined surfaces", Angew. Chem. Int. Ed. 49, 9699-9701 (2010).

[29] C. Picioreanu, M. Van Loosdrecht, and J. Heijnen, "Two-dimensional model of biofilm detachment caused by internal stress from liquid flow", Biotechnol. Bioeng. 72, 205-218 (2001).

[30] H.-C. Flemming, J. Wingender, and U. Szewzyk, Biofilm highlights, Springer Series on Biofilms, 1st edition (Springer-Verlag, Berlin Heidelberg) (2011).

[31] P. Stoodley, Z. Lewandowski, J. D. Boyle, and H. M. Lappin-Scott, "Structural deformation of bacterial biofilms caused by short-term fluctuations in fluid shear: An in situ investigation of biofilm rheology", Biotechnol. Bioeng. 65, 83-92 (1999).

[32] P. Stoodley, S. Wilson, R. Cargo, C. Piscitteli, and C. J. Rupp, "Detachment and other dynamic processes in bacterial biofilms", in Surfaces in Biomaterials 2001 Symposium Proceedings, 189-192 (Surfaces in Biomaterials Foundation, Minneapolis, USA) (2001).

[33] S. Seltzer, I. B. Bender, J. Smith, I. Freedman, and H. Nazimov, "Endodontic failures-an analysis based on clinical, roentgenographic, and histologic findings", Oral. Surg. Oral. Med. O. 23, 500-516 (1967).

[34] H.-C. Flemming, T. R. Neu, and D. J. Wozniak, "The eps matrix: The "house of biofilm cells"”, J. Bacteriol. 189, 7945-7947 (2007).

[35] I. Portenier, T. Waltimo, D. Orstavik, and M. Haapasalo, "The susceptibility of starved, stationary phase, and growing cells of enterococcus faecalis to endodontic medicaments", J. Endodont. 31, 380-386 (2005).

[36] L. Kuznetsova and W. Coakley, "Applications of ultrasound streaming and radiation force in biosensors", Biosens. Bioelectron. 22, 1567-1577 (2007).

[37] A. Zijlstra, “Acoustic surface cavitation”, Ph.D. thesis, University of Twente (2011).

[38] D. Fernandez Rivas, L. Stricker, A. G. Zijlstra, H. J. G. E. Gardeniers, D. Lohse, and A. Prosperetti, "Ultrasound artificially nucleated microbubbles and their sonochemical radical production", Ultrason. Sonochem. Accepted, xx (2012).

[39] C. D. Meinhart, S. T. Wereley, and M. H. B. Gray, "Volume illumination for two-dimensional particle image velocimetry", Meas. Sci. Technol. 11, 809-814 (2000).

[40] S. V. Van der Waal, L. W. M. Van der Sluis, R. A. Özok, R. A. M. Exterkate, P. R. Wesselink, and J. J. De Soet, "The effects of hyperosmosis or high ph on a dual-species biofilm of pseudomonas aeruginosa and enterococcus faecalis: an in vitro study", Int. Endod. J. 44, 1110-7 (2011).

[41] A. N. Jatariu-Cadinoiu, M. Popa, S. Curteanu, and C. A. Peptu, "Covalent and ionic co-cross-linking - an original way to prepare chitosan-gelatin hydrogels for biomedical applications”, J. Biomed. Mater. Res. 98A, 342-350 (2011).

[42] D. Fernandez Rivas, M. Ashokkumar, T. Leong, K. Yasui, T. Tuziuti, S. Kentish, D. Lohse, and H. J. G. E. Gardeniers, "Sonoluminescence and sonochemiluminescence from a microreactor", Ultrason. Sonochem. 19, 1252-1259 (2012).

[43] J. Israelachvili and R. Pashley, "Measurement of the hydrophobic interaction between two hydrophobic surfaces in aqueous electrolyte solutions", J. Colloid Interface Sci. 98, 500-514 (1984).

[44] A. Freitas and M. Sharma, "Effect of surface hydrophobicity on the hydrodynamic detachment of particles from surfaces", Langmuir 15, 2466-2476 (1999).

[45] R. Vos, M. Lux, K. Xu, W. Fyen, C. Kenens, T. Conard, P. Mertens, M. Heyns, Z. Hatcher, and M. Hoffman, "Removal of submicrometer particles from silicon wafer surfaces using hf-based cleaning mixtures", J. Electrochem. Soc. 148, G683-G691 (2001). 
[46] R.-H. Yoon, D. Flinn, and Y. Rabinovich, "Hydrophobic interactions between dissimilar surfaces", Journal of Colloid and Interface Science 185, 363-370 (1997).

[47] K. Mittal, Adhesion measurement: Recent progress, unsolved problems, and prospects, 5-16, in [60] (1976).

[48] P. Benjamin and C. Weaver, "Adhesion of metal films to glass", Proc. R. Soc. London, Ser. A 254, 177-183 (1960).

[49] D. E. Riemer, "High-adhesion thick-film gold without glass of metal-oxide power additives.", IEEE transactions on components, hybrids, and manufacturing technology CHMT-8, 474-480 (1985).

[50] J. Dini, Electrodeposition: the materials science of coatings and substrates (William Andrew) (1993).

[51] R. Weast, ed., CRC Handbook of Chemistry and Physics, 52 edition (CRC PRESS) (1971).

[52] J. Minford, Durability evaluation of adhesive bonded structures, chapter 9, 239-284 (Plenum Press) (1991).

[53] Y. H. An and R. J. Friedman, Handbook of bacterial adhesion, 1st edition (Humana Press, Totowa, NJ, USA) (2000).

[54] W. M. Dunne Jr., "Bacterial adhesion: Seen any good biofilms lately?", Clin. Microbiol. Rev. 15, 155-166 (2002).

[55] S. Bayoudh, A. Othmane, L. Mora, and H. Ben Ouada, "Assessing bacterial adhesion using dlvo and xdlvo theories and the jet impingement technique”, Colloids Surf., B 73, 1-9 (2009).

[56] P. C. Y. Lau, J. R. Dutcher, T. J. Beveridge, and J. S. Lam, “Absolute quantitation of bacterial biofilm adhesion and viscoelasticity by microbead force spectroscopy”, Biophys. J. 96, 2935-2948 (2009).

[57] C. Mayer, R. Moritz, C. Kirschner, W. Borchard, R. Maibaum, J. Wingender, and H.-C. Flemming, "The role of intermolecular interactions: studies on model systems for bacterial biofilm”, Int. J. Biol. Macromol. 26, 3-16 (1999).

[58] G. Sagvolden, I. Giaever, and J. Feder, "Characteristic protein adhesion forces on glass and polystyrene substrates by atomic force microscopy”, Langmuir 14, 5984-5987 (1998).

[59] C.-D. Ohl, M. Arora, R. Ikink, N. De Jong, M. Versluis, M. Delius, and D. Lohse, "Sonoporation from jetting cavitation bubbles", Biophys. J. 91, 4285-4295 (2006).

[60] K. Mittal, ed., Adhesion measurement of thin films, thick films, and bulk coatings : a symposium presented at ASTM headquarters, American Society for Testing and Materials (Philadelphia : American Society for Testing and Materials) (1976).

[61] J. Vossen, Measurement of Film-substrate bond strength by laser spallation, 122-131, in Mittal [60] (1976). 


\section{A AFM scratch test for the adhesion of solid hard mate- rials}

\section{A.1 Experimental setup}

The adhesion of gold $(\mathrm{Au})$ and platinum $(\mathrm{Pt})$ was determined by scratching these deposited layers using the same AFM as before, but with a different spherical cap tip (Micromasch NSC35, spring constant of $14 \mathrm{~N} / \mathrm{m}$, resonant frequency $315 \mathrm{kHz}$ ) in contact mode. For these measurements, the surface was scanned continuously over a $20 \mu \mathrm{m} \times 20 \mu \mathrm{m}$ areas with the contact force increased gradually every $5 \mu \mathrm{m}$. Once the metal layer began to tear, the cantilever was lifted and moved elsewhere on the substrate, before the process was repeated. Several repeated measurements were carried out.

\section{A.2 Results}

Only Au and Pt could be removed with this AFM scratch test. The required removal forces were found to be $22.6 \pm 0.5 \mu \mathrm{N}(\mathrm{Au})$ and $44.0 \pm 1.5 \mu \mathrm{N}(\mathrm{Pt})$, which, using a removal width of $R=10 \mathrm{~nm}$, correspond to peeling strengths of $2260 \pm 50 \mu \mathrm{N} / \mathrm{m}$ $(\mathrm{Au})$ and $4400 \pm 150 \mu \mathrm{N} / \mathrm{m}(\mathrm{Pt})$.

The gold and platinum layers delaminated differently to each other (Figure 7.9). For the gold layer, once the metal had been compromised it continued to delaminate from the glass substrate even if the contact force was reduced to less than $0.5 \mu \mathrm{N}$. However, the platinum delaminated in a stick-slip, patchy fashion, in any case, so lowering the contact force by only a few percent led to no further damage. Even at a constant force of $44.0 \pm 1.5 \mu \mathrm{N}$, the scratch was intermittent. The scratch widths were increased to $90 \mu \mathrm{m}$ for platinum to better see the effect.

The required forces and peeling strengths for metal removal given above were measured on defect-free areas of the surface. It is interesting to note that the surfaces peeled at much lower applied forces if the AFM probe collided with an opticallysized $(\sim 10 \mu \mathrm{m})$ asperity. For the gold, the required force and peeling strength were dramatically reduced to approx. $2.1 \mu \mathrm{N}$ and $210 \mathrm{~N} / \mathrm{m}$, respectively, whilst those for platinum were reduced to approx. $20 \mu \mathrm{N}$ and $2000 \mathrm{~N} / \mathrm{m}$.

\section{B Macroscopic scratch test for the adhesion of solid hard materials}

\section{B.1 Experimental setup}

A hardness tester kit (SP0010, Thermimport Quality Control) and a cross-cut adhesion tester kit (CC2000 SP1690, Thermimport Quality Control) were used to analyze 

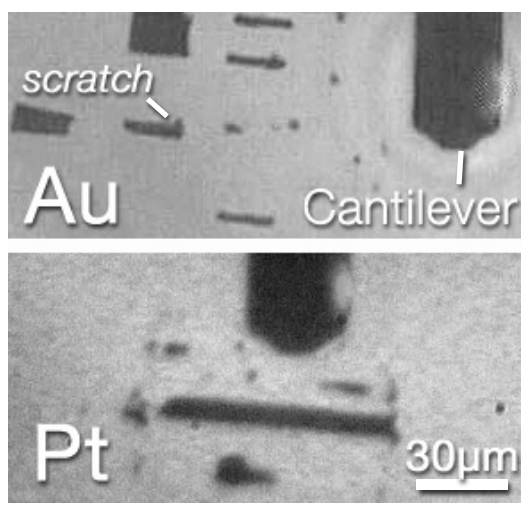

Figure 7.9: The top image is for the gold sample and several scratch lines can be seen. Each one is $20 \mu \mathrm{m}$ wide. The lower image is for the platinum sample. As a guide to the eye, the AFM probe mount is $30 \mu \mathrm{m}$ wide.

the adhesion strength of the solid hard materials. The hardness tester kit consisted of a Tungsten-Carbide tip with $1 \mathrm{~mm}$ diameter and a spring with 0-300 g load capacity and can be used to measure the wear (by scratching) or fail resistance of coatings, plastics, etc. The cross-cut adhesion tester kit is based on ISO 2409 Standards and provides a semiquantitative measurement of adhesion. First, perpendicular cuts with parallel blades were performed through the layers, touching the glass slide; afterwards a dedicated adhesive tape is attached and, when detached, it can remove weakly adhered patches of each layer. According to the amount of removed material, a classification of surface adhesion quality is given with a score between 0 and 5; 0 being the stronger attached where none of the squares is detached after peeling off the tape.

\section{B.2 Results}

The hardness tester is a simple semi-quantitative test for surface hardness in which all materials layers were scratched with $1 \mathrm{~g}$ (minimum setting that could be used).

The scratch test of the different solid hard materials used showed a marked difference between the metal and organic layers (Figure 7.10). When scratching the organic layers (thicker and more brittle) we observed small parts detaching and it was not possible to cut well defined squares as in the case of the metals.

$\mathrm{Au}$ and Pt were the weakest attached materials falling in the 5-and-4 and 4-and-3 category respectively. For Au layers we observed that the layer flaked in large ribbons and along the edges where most squares were removed (more than $35 \%$ of the total area). For Pt we saw that most squares were partially or totally removed (more than $15 \%$ of the total area). For $\mathrm{Cr}$ and $\mathrm{Ti}$ we classified it as 1 -and- 0 as we observed very small flakes of the coating at the intersections of the cuts and no square could be removed (less than $5 \%$ of the area removed).

For AZ and Olin we found that some chunks were removed in a way that could be cataloged 2-and-1, but the squares were not neatly formed as these brittle layers 
cracked; see Figure 7.10

Summarizing, the qualitative adhesion of the solid hard materials can be arranged, in order of weakest to strongest, as: $\mathrm{Au}<\mathrm{Pt}<$ Olin and $\mathrm{Az} 96<\mathrm{Ti}$ and $\mathrm{Cr}$.

This qualitative quantification is consistent with values provided in the literature using other techniques. For example, for the metals, a laser spallation technique was used to find a direct correlation between the bond strength on silicon oxide substrates with the free energy of oxide formation of the arriving metal vapor for both evaporated and sputtered metals [61]. The energies at which the films detached were: Au $\left(25 \mathrm{~J} / \mathrm{cm}^{2}\right), \mathrm{Cr}\left(75 \mathrm{~J} / \mathrm{cm}^{2}\right)$ and $\mathrm{Ti}\left(125 \mathrm{~J} / \mathrm{cm}^{2}\right)$, showing the same relative adhesion strength as found in the present study.

\section{C Ultrasonic bath test for the adhesion of solid hard ma- terials and comparison with current setup}

\section{C.1 Experimental setup}

A commercial ultrasonic bath (VWR) operating at $20 \mathrm{kHz}$ was used to test the adhesion strength of the layers with a conventional ultrasonic apparatus and a comparison of the removal capabilities for each deposited sample was performed.

\section{C.2 Results}

With the ultrasonic bath, no material was removed after 10 min of sonication. All samples were individually placed in a conventional configuration of a glass beaker of $200 \mathrm{~mL}$ (Schott) glued to a microscope glass slide (see Figure 7.11 Test A).

As an extreme test we placed each sample facing down, directly against the bottom surface of the ultrasonic bath. For Au and $\mathrm{Pt}$ we observed surface removal without localization and for $\mathrm{Cr}$ or Ti we saw no removal at all (see Figure 7.11 Test B).

\section{D Contact angle measurement of solid hard materials}

\section{D.1 Experimental setup}

The advancing and receding contact angles of a sessile drop of tapwater on the gold-, platinum-, chromium- and titanium-coated glass chips in air was determined using a commercial contact angle goniometer (OCA30, Dataphysics, Filderstadt, Germany). The advancing and receding contact angles were determined automatically thrice by the contact angle goniometer during volume change of a drop of volume $10 \mu \mathrm{L}$ at a rate of $0.25 \mu \mathrm{L} / \mathrm{s}$. The non-clean conditions in the ultrasound setup were mimicked by using tap water and by not cleaning the chips before contact angle measurement. 

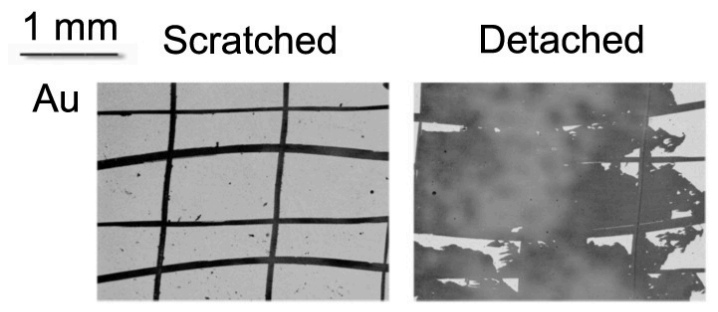

Pt
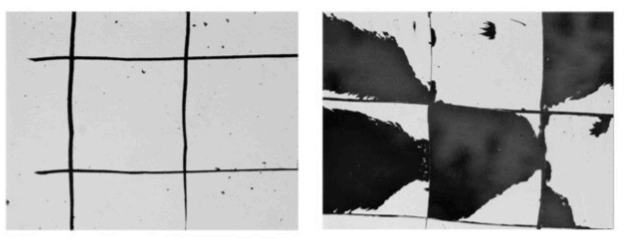

$\mathrm{Ti}$
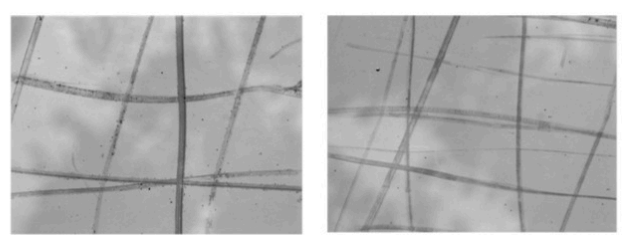

\section{$\mathrm{Cr}$}
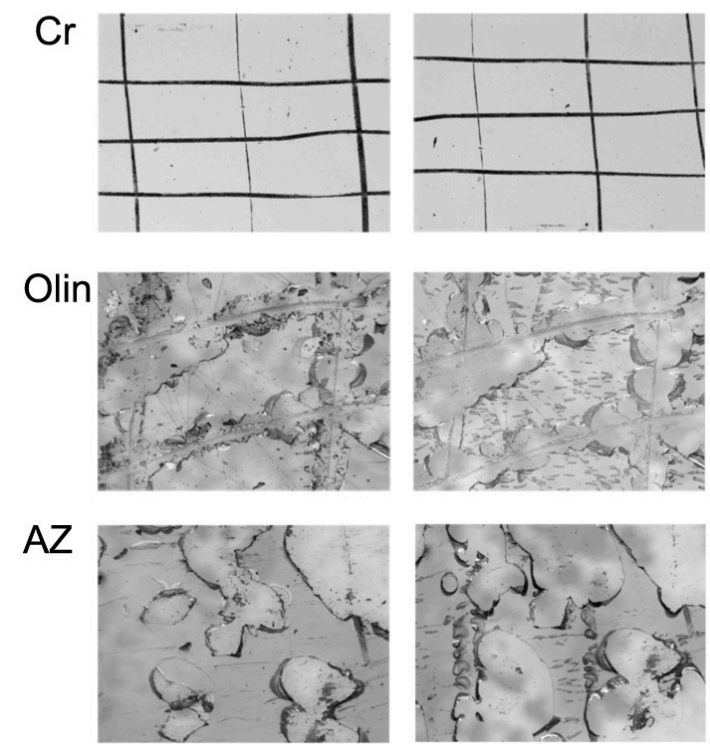

Figure 7.10: The left column shows the various deposited layers after the cuts are performed. The right side shows the same region after peeling off the adhesive tape. 
Figure 7.11: Experimental conditions provided to test adhesion strength for all layers; in this case for Gold deposited on a glass substrate. Test A shows how the glass substrate was glued to a glass slide and inserted in a glass beaker filled with water as normally cleaning US baths are used. After 10 min no material was removed in any case. Test $B$ shows the extreme conditions used to be able to remove material (only $A u$ and Ti) but no localization of the removed material.

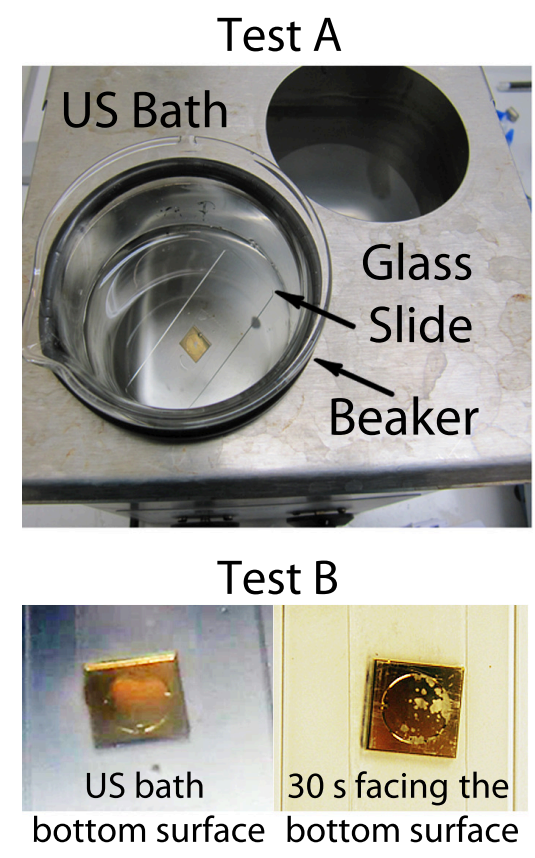

\section{D.2 Results}

The results are included in Table 7.1. Au and Pt were found to have a larger receding contact angle than the other solid hard materials.

\section{E Hydrodynamic characterization of the viscoelastic prop- erties of the the hydrogel}

\section{E.1 Experimental setup}

The properties of the hydrogel used in this study have been measured by determining the strain induced by the stress exerted by the flow from a needle. When the flow through the needle is driven with a positive pressure, a jet is created, which has a stagnation pressure $P$ of $P=\frac{1}{2} \rho u^{2}$, where $\rho$ is the density of the liquid (water: 1000 $\mathrm{kg} / \mathrm{m}^{3}$ ) and $u=Q / A$ is the average velocity, estimated from the flow rate $Q$ and the needle orifice $A$. The setup consisted of a $22 \mathrm{G}$ needle (Terumo, Leuven, Belgium; internal diameter of $0.413 \mathrm{~mm}$ ) positioned at a distance of $300 \mu \mathrm{m}$ above the hydrogelcovered surface, through which a flow with flow rates in the range $0.01-1 \mathrm{~mL} / \mathrm{min}$ were created by a syringe pump (NE1010, New Era Pump Systems, Wantagh, NY, USA). By recording (LM165 camera, Lumenera) the displacement of particles embedded in the hydrogel that appeared to be near the stagnation region of the jet, the 
Young modulus of the hydrogel can be estimated.

Using the same needle but with a negative pressure, a pulling force is exerted on the hydrogel. Parts of the hydrogel detach (sloughing) from the bulk hydrogel when the suction pressure exceeds the cohesive and adhesive forces of the hydrogel. The suction pressure can be estimated roughly from the Bernoulli pressure along the streamlines of the flow toward the needle orifice.

\section{E.2 Results}

The hydrogel, when subjected to stagnation pressure from a jet generated with a needle, displayed elastic behavior for flow rates higher than $0.01 \mathrm{~mL} / \mathrm{min}$, and additional viscous behavior for flow rates higher than $0.1 \mathrm{~mL} / \mathrm{min}$. By tracking the displacement of embedded particles in the elastic regime, the Young modulus of the hydrogel was estimated to be of the order of $10^{-1} \mathrm{~Pa}$. When using a negative pressure (suction) through the needle, sloughing was observed to occur for flow rates above 0.2 $\mathrm{mL} / \mathrm{min}$, from which the adhesive and cohesive strengths are estimated to be of the order of $10^{-1} \mathrm{~Pa}$.

The mechanical properties of the biofilm could not be determined, because its limited thickness prohibited proper recording of its deformation and the other tests used for the organic materials and metals are not applicable. However, overall the biofilm appeared to be stiffer and better attached to the substrate than the hydrogel was. This agrees with the typical range of values of the Young's modulus and cohesive and adhesive strengths for biofilms given in literature [29-32, 54-56], which are typically two or three order of magnitude higher than those measured for the hydrogel.

\section{Supplementary material}

(Online at http://stilton.tnw.utwente.nl/rootcanalcleaning/Gallery_of_Irrigant_Motion)

Movie 1. Removal of a deposited layer of gold by cavitating microbubbles, originating from two pits. After increasing the power (at $t \approx 8 \mathrm{sec}$ ), the bubble clouds start to move toward each other and remove an area in-between the two pits. Recording speed is $50 \mathrm{fps}$.

Movie 2. Removal of a deposited layer of photoresist (AZ96). Recording speed is 50 fps.

Movie 3. Removal of a deposited layer of biofilm (black dots). Recording speed is 50 fps. 


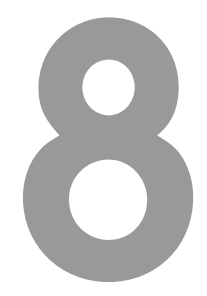

\title{
Erosion Evolution in Mono-crystalline Silicon Surfaces Caused by Acoustic Cavitation Bubbles * *
}

\begin{abstract}
The early stages ( $<180 \mathrm{~min}$ ) of cavitation erosion of silicon surfaces were studied for three different crystallographic orientations. We introduce a quantity defined as the ratio of non-dimensional eroded area to the number of pits, $\alpha_{p}$, to evaluate the evolution of erosion among the different substrates used. Different erosion evolution was observed for (100), (110) and (111) silicon surfaces when exposed to cavitation bubbles generated by an ultrasound signal of $191 \mathrm{kHz}$. (100) silicon substrates showed the most erosion damage, with an eroded area 2.5 times higher than the other two crystallographic orientation substrates after $180 \mathrm{~min}$ sonication. An apparent incubation period of $50 \mathrm{~min}$ was measured. The number of erosion pits increased constantly for (110) and (111), but for (100) no increase was detected after $120 \mathrm{~min}$. The collapse of a spherical bubble was simulated using an axisymmetry boundary integral (BI) method. The calculated velocity of the jet from the collapsing bubble was used to estimate the pressure $P$ that is induced by the jet upon impact on the silicon substrate.
\end{abstract}

${ }^{*}$ To be submitted as: David Fernandez Rivas, Joris Betjes, Bram Verhaagen, Wilco Bouwhuis, Ton C. Bor, Detlef Lohse and Han J.G.E. Gardeniers, to J. Appl. Physics, 2012. 


\subsection{Introduction}

\subsubsection{From cavitation bubbles to erosion}

In 1894 Sir. John I. Thornycroft and Sydney W. Barnaby found bubble formation (cavities) on spinning propeller and speculated that it was connected to vibration and erosion of the propeller. Later on, the English Royal Navy approached John William Strutt (Lord Rayleigh) to study the causes for the damage found on boat and submarine propellors moving at high speeds [1--3]. Rayleigh confirmed that this damage was due to cavitation bubbles. Cavitation is defined as the growth and collapse of a gaseous void when an external negative pressure is applied to a liquid volume [4, 5]. The ship propellers rotate fast enough to reduce the pressure in the liquid near the blades below the water vapor pressure, leading to the formation of bubbles. The subsequent bubble collapse can be so violent that materials as hard as steel can be severely damaged, which is referred to as cavitation damage. In the past decades, cavitation damage has been of interest in engineering since it is an undesirable effect in, among others, artificial heart valves, hydraulic machinery, and bearings [6-8]. The mechanical effects of ultrasound cavitation have found several "greener" applications in the last decade, such as localized cleaning of surfaces, the development and optimization of new coatings, surface functionalizations, nanofoams and several other used in solar cells, imaging sensors, mechanical parts and hip prostheses [9-18]. As an example, it is possible to make Si surfaces porous by exposure to ultrasound cavitation which can be used as photoluminescent structure, additionally reducing aging effects of the Si surface [14, 19-21].

Cavitation is influenced by several parameters such as saturating gas, pressure amplitude and temperature, which are interconnected and are hard to fine-tune [22]. In practical situations, bubbles frequently nucleate from defects on the wall of the container or in dissolved particles, where small pockets of gas are trapped (also termed heterogeneous cavitation). Controlled formation of cavitation bubbles can be achieved by using for example a laser, shockwaves, or ultrasound equipment [23. 28]. Here we use an ultrasound device employing micropits that has been shown recently [10] to enable the control of the location and amount of cavitation and the surface modification effects on a millimeter scale.

Cavitation bubbles display a rich behavior. When bubbles undergo stable cavitation, acoustic streaming and shear forces on the surrounding medium are generated. On the other hand, when the bubble collapse is more inertial, high fluid velocities, high pressures and temperatures, and shock wave emission are present. The presence of a boundary or other bubbles breaks the symmetry around an otherwise spherical collapsing bubble, leading to a high-velocity jet. The velocity of such a jet can be of the order of $100 \mathrm{~m} / \mathrm{s}$ [29, 30].

Secondary effects of inertial bubble collapse include bubble fragmentation, splash- 
ing and deformation of the walls nearby the collapsing bubble [4, 11, 24, 30,-34]. A splashing effect has been described as the damage caused by the spherical collapse of microbubbles near the surface, which are the result of after-bounces or second bubble collapses. After a micro-jet hits the surface, it spreads inside the boundary layer and bubbles are thought to be generated due to friction; the collapses of these small, spherical bubbles lead to powerful shock waves [31].

The majority of literature agrees that the destructive action of cavitation bubbles is due mainly to two phenomena: the generation of the high-speed liquid jet directed towards the (solid) boundary and the emission of shock waves upon the collapse of the bubble. Another effect was suggested by Shutler \& Mesler [35] as early as 1965 , in which the bubble surface collapses directly towards the close-by boundary, increasing the damage capability of the shock waves, jetting or any other damaging effect.

Frequency is an important parameter that will determine several effects of cavitation such as the size of bubbles, surface modifications, chemical effects and light emission, to name a few [13, 22, 36, 37]. At lower frequencies (ca. $20 \mathrm{kHz}$ ), physical surface modification occurs mainly due to the effect of energetic micro-jetting, modifying the surface morphology with high weight loss [13]. For higher frequencies (up to $150 \mathrm{kHz}$ ) the pressure field, luminescence, and erosion location coincide with less erosion as a result related to damped cluster collapse [38]. The concerted collective action of cavities is known to give rise to pressures at the target surface as high as $900 \mathrm{MPa}$ [39].

When a surface is impacted (jetting or shockwave), stress waves are generated and propagate in the solid, which can generate damage not only to the surface but also to the bulk [18, 40]. Two basic types of stress waves exist: body and surface waves. Compression waves propagate in the body, the shear and surface waves are of the Rayleigh type. The orientation and velocity of a liquid jet and the severity of the collapse and subsequent effect will depend on the impedance of the material loaded and its stand off distance $\gamma$ from the boundary. Near a hard boundary, the jet will form towards the boundary, whereas near a soft boundary, the jet may form away from the wall and thereby pull on the wall [30].

Ohl et al. [41] were able to locate the origin of two shock waves as the separate emission of a jet-induced and a bubble-collapse-induced shock wave. A shock wave is generated by the impact of the jet tip onto the lower bubble wall (jet shock wave) [30]. The pressure acting on a rigid wall due to a shock wave can be of the order of $10 \mathrm{GPa}$ [34]. During cloud rebound after the collapse of a hemispherical cloud of bubbles close to a rigid boundary, secondary shock wave emissions occur.

It has been shown that the spherical shock waves arising in a liquid during cavitation bubble collapse lead to formation of deep needle-like pits on the solid surface [39]. This damage is caused by the spallation due to interference of rarefaction waves, 
and it was found for several materials such as steel and duraluminum. Spallation was defined as dynamic damage taking place upon focusing or interference of rarefaction waves. Several spall cracks in the material yield fragmentation and detachment of material grains, forming craters.

\section{Cavitation erosion in Silicon}

The effects of microbubble cavitation close to silicon surfaces in a micro-sono-reactor has been described in previous publications [27, 28, 42]. Within 5 minutes of exposure to cavitation bubbles nucleated from pits, a region of damaged silicon substrate was observed near the region where bubbles collapsed. Remarkably, the shape of the individual damage sites coincided with the $\{111\}$ crystal planes of the crystalline structure of the silicon (100) wafers used. In connection to surface damage, the emission of shock waves by collapsing bubbles and jetting towards the silicon surfaces was highlighted as possible causes. The current study will attempt to obtain more details on erosion evolution in silicon substrates. Additionally, the setup may be used not only to test the erosion on the surface with the etched pits, but in a similar way as presented before [10], on another surface in the vicinity of the bubble generation.

Perhaps the pioneering work with strong similarities to the work we present here was published in 1965 by Howkins [25]. An air bubble was trapped in a hole (1 $\mathrm{mm}$ diameter and depth) drilled into a surface that later is immersed in water and vibrated. When exposed to ultrasound at $20 \mathrm{kHz}$ with degassed water and water saturated with air, the erosion in a nearby brass surface was studied, giving erosion even at ultrasound amplitudes at which, in the absence of the bubble, no erosion took place.

More recently [18] the physicochemical behavior of crystalline silicon (100) under acoustic cavitation was investigated in water sparged with argon. $\mathrm{Ar}^{*}$ emissions generated by mechanoluminescence (emission of light caused by mechanical action on a solid [43]) were detected. Besides physical and chemical changes in the Si surface that altered wetting properties and topology, phase transformations of the Si lattice were studied with Raman spectroscopy and Transmission Electron Microscopy, TEM. Long incubation periods of erosion were found with cracks and defect cleavages aligned with the crystallographic lattice in agreement with the brittle nature of $\mathrm{Si}$ at room temperature. Interestingly, local disorder damage was found in line with plastic deformations [18].

Here we investigate erosion of several silicon surfaces. Silicon has a diamond face-centred cubic crystal structure (fcc). Slip in silicon occurs on $\{111\}$ slip planes with Burgers vectors of $\mathrm{a} / 2<1 \overline{1} 0>$ and $\mathrm{a} / 6<11 \overline{2}>$ types [44]. The angles $(\phi)$ between planes of the family $\{100\}$ and $\{110\}$ are $45^{\circ}$ or $90^{\circ}$. Between $\{100\}$ and $\{111\}$ planes, the intersection angle is $54.7^{\circ}$. Furthermore, $\{111\}$ and $\{110\}$ planes intersect under $35.3^{\circ}, 90^{\circ}$ or $144.7^{\circ}$ [45]. Figure 8.1 illustrates these orientations 
schematically.

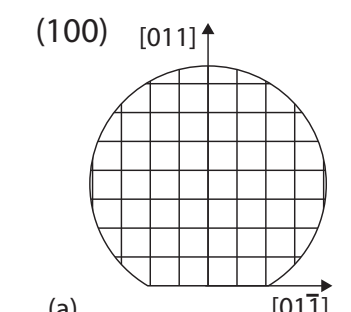

(a)

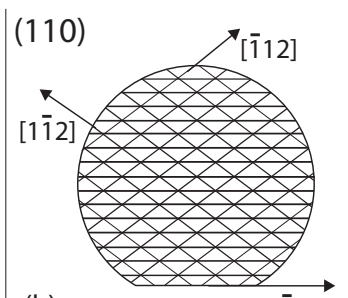

(b)

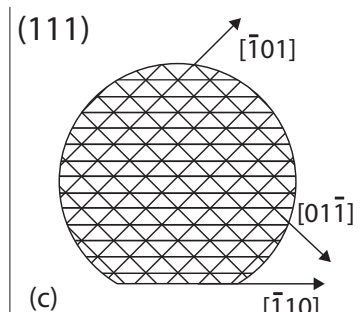

Figure 8.1: Sketch of the crystallographic plane configuration in mono-crystalline silicon for (a) (100), (b) (110) and (c) (111). All lines are the intersecting $\{111\}$ planes with each corresponding specific top surface plane.

\section{Erosion evolution}

Studies of cavitation erosion have covered a wide variety of materials and conditions; from ductile metals, brittle plastics, ceramics and soluble materials to glass [11, 40, 46-48]. All materials have intrinsic defects in its surface and bulk. When exposed to cavitation activity, regularly an incubation period occurs where small deformations (erosion pits) appear on the surface after short duration exposure, or the existing defects start to grow. These defects can serve as heterogeneous nucleation sites and promote cavitation erosion. As exposure to cavitation continues, different behaviors are observed depending on the nature of the material. The erosion can accelerate and eventually reach a stable erosion rate [31, 49, 50].

When cyclic stresses of a given load are applied for a certain time, most materials degrade and can suffer premature failure. This phenomenon is known as fatigue and is the most commonly experienced form of structural failure, yet it is one of the least understood for single crystalline silicon [51].

Brittle materials, such as single crystal silicon at room temperature, have very limited dislocation mobility, making the possibility of cyclic fatigue failure not obvious. The durability and reliability assessment of microscale devices (air bags, blood pressure sensors, and jet cartridge nozzles) has driven studies on the fatigue effect on materials used in the semiconductor industry [52]. The fatigue damage accumulation in silicon can take place with the detachment of inclined $\{111\}$ planes on the fracture surface when energy is dissipated, forming cleavage planes [51, 52].

The cracking behavior of $\mathrm{Si}$ is not that well reported in the literature. The corresponding mechanisms of fatigue in brittle materials, such as the structural films commonly used in MEMS, are quite different from the generation and motion of 
dislocations and the accumulation of plastic deformation in metals. The $\{111\}$ and $\{110\}$ planes are known to be preferred cleavage planes, which means that cracks propagate preferentially along them. Reported values of the fracture toughness are given in Table 8.1, but do not differ much from each other.

The influence of the environmental conditions on $\mathrm{Si}$ fatigue in air and water have also been tested with the conclusion that the fracture surface in water consists of several fracture planes (failed by several cracks), whereas the one in air is a single plane (single crack) [53]. Apparently, water influences the oxidation of the silicon surface and accelerates the erosion-fatigue effects. A similar effect occurs for glass surfaces where silanol forms [54].

Ultrasound cavitation can have an etching effect induced by the formation of monohydrides to the detriment of multihydride formation. Ultrasound cavitation can also lead to porous layers with decreased oxidation and hydrogenation [20].

\section{Quantifying cavitation erosion}

Materials erosion is a complex phenomenon in itself, and due to the rich behavior of bubbles, we find an even more complex correlation in cavitation erosion. Methods for quantifying erosion strongly depend not only on the device, frequency, material, and exposure time, but also on the laboratory conditions available in each research group and the degree of understanding of the physico-chemical processes involved. A popular method to quantify or map cavitational erosion effects has been the damage of aluminum foils [30, 31], but this should be considered mainly as a way to find regions with maximum erosion potentials, and the results cannot be extrapolated to other materials. The relation between impact load by collapsing cavitation bubbles and erosion damage can be addressed by measuring impact loads and erosion damage with sophisticated equipment [55].

Several single-number parameters like the volume loss, the mass loss, and the mean and maximum depth of erosion penetration are determined after exposure to cavitation in order to quantify the amount of erosion that has taken place. Additionally, the instantaneous erosion rate, the mean depth of erosion penetration rate, the incubation period and the time needed to achieve the maximum damage rate are used to characterize a given period of erosion progress.

Efforts to standardize existing erosion test methods have had limited success [56]. As the damage rates of different materials depend on so many factors, the realistic loading of the material under field conditions is usually unknown. Often ultrasonic horns or baths are used, but they have limitations in the range of frequencies, power and pressure values. Also, as erosion at early stages is so small, it is not possible to measure erosion by weighing the samples before and after ultrasound exposure. Here, we demonstrate that the ultrasound device with micropits can be used effectively to study the erosion of silicon; other materials may be used as well. Using 
high-resolution scanning electron microscopy (SEM) imaging, information on the evolution and extend of cavitation erosion can be obtained in a quantitative way, allowing for comparison of the erosion of different materials.

Varvani [57] proposed a model to obtain the number of cycles for fatigue failure of silicon to occur, in which the total fatigue damage is accumulated as the number of cycles progresses. Here, we attempt to relate the number of cycles to erosion by measuring the amount of erosion after a certain time and correlating that to the calculated expected number of cavitation bubbles.

\subsection{Experimental section}

\subsubsection{Experimental setup}

Experiments were conducted with $1 \times 1 \mathrm{~cm}$ square micromachined chips silicon single crystalline with orientation $<100>,<110>$ and $<111>$ (Okmetic, Vantaa, Finland). The chips were diced from different p-type polished wafers with a smooth surface $(3.46-4.22 \AA)$. Two pits were micromachined in the surface with an interpit distance of $100 \mu \mathrm{m}$; each pit has a diameter of $30 \mu \mathrm{m}$ and a depth of $\sim 10 \mu \mathrm{m}$. Further details on the fabrication of the micromachined pits on the substrates can be found elsewhere [27, 28, 42].

The glass cavitation cell, in which the substrates were placed, was glued to a piezo element and details can be found elsewhere [27, 28, 42]. An Agilent 33250A $80 \mathrm{MHz}$ wave generator provided a $191 \mathrm{KHz}$ sinusoidal signal, which was amplified by a Sony TA-FB 740FBR amplifier to $0.07 \pm 0.01 \mathrm{~W}$, providing an acoustic density of $0.14 \mathrm{~W} / \mathrm{cm}^{2}$. The bottom of the piezo element was in contact with a Marlow Industrial Peltier-element to keep the temperature at $23 \pm 1^{\circ} \mathrm{C}$. A heat sink and fan were placed underneath the piezo element to remove excess heat. A glass slide and rubber ring were placed over the reaction cell to reduce evaporation of the liquid during the experiments. A Multimetrix DMM220 multimeter connected to a 0.2 mm diameter T-type thermocouple was used to measure the temperature within the reaction cell. The current and voltage supplied to the piezo element were monitored with a HAMEG HM 8115-2 power meter.

\subsubsection{High-speed visualization setup for bubble dynamics}

The generation of cavitation from the micropits was visualized using two cameras. One was the ultra-high speed camera facility Brandaris 128 [58], which recorded 6 movies (spaced $20 \mathrm{~ms}$ apart) of 128 frames at a frame rate of approximately 10 Mfps. The other was the Photron, model SA1.1 with framerates up to $500 \mathrm{kfps}$. A microscope (BX-FM, Olympus) provided $20 \times$ magnification; illumination was provided in dark-field mode using a Xenon flash source. 
A second system reported before [28, 59], able to capture single snapshots with a short exposure time was used to image shockwave emissions. The camera was a Lumenera LM165 with a sensitive Sony EXview HAD CCD sensor. The Olympus microscope was equipped with two long working distance (WD) objectives, the LMPLFLN 10×, (WD $21 \mathrm{~mm} / \mathrm{NA}=0.25$ ) and the SMPLFLN 20× (WD $25 \mathrm{~mm}$ $/ \mathrm{NA}=0.25$ ) both Olympus. A bright laser-induced fluorescence pulse of $7 \mathrm{~ns}$ duration (full width at half maximum) was used for illumination, amounting to about one thousandth of the acoustic period, providing high-resolution images without motion blur.

\subsubsection{Numerical modeling of single bubble collapse near a solid wall}

The collapse of a spherical bubble was simulated using an axisymmetric boundary integral (BI) method, see Appendix 8.A for details. The BI simulation tracked the interface of the bubble up to the point where the two opposite interfaces of the axisymmetric bubble touched each other. The velocity difference of these two interfaces at this instant was used as an approximation of the velocity $V$ of the jet from the collapsing bubble.

The pressure $P$ that is induced by the jet upon impact on the silicon substrate can be estimated calculating the water hammer pressure [60]:

$$
P=\frac{\rho_{1} C_{1} \rho_{2} C_{2} V}{\rho_{1} C_{1}+\rho_{2} C_{2}}
$$

with $\rho$ the density and $C$ the speed of sound in the liquid (subscript 1 ) and the silicon (subscript 2). The water hammer pressure is associated with the shock wave induced by the impact of a liquid cylinder onto a solid surface [61].

The wall shear stress is estimated from the Glauert solution for the wall jet, as outlined by Ohl et al. [62], and evaluated at a distance of one jet diameter away from the center of the jet. The jet diameter is estimated to be a fraction of the maximum radius of the bubble: $R_{\max } / 60$.

\subsubsection{Silicon surfaces selection and cavitation exposure}

Table 8.1 summarizes the most important mechanical properties of $\{100\},\{110\}$ and $\{111\}$ cleavage planes of silicon. The differences reported by different authors are mainly due to the ways the measurements are carried out and by the exact lattice orientation which can vary from one manufacturer to another [63-68].

All substrates were cleaned using a VWR ultrasonic cleaner at $20 \mathrm{kHz}$ for five minutes, Olympus lens cleaner tissues and three chemicals (acetone, alcohol and milliQ water). The beaker was moved constantly in order to avoid stable bubbles collapsing against the silicon substrate. The cleaned substrates were dried with inert 
Table 8.1: Physical properties of mono-crystalline silicon crystal planes.

\begin{tabular}{|c|c|c|c|}
\hline Crystal planes & $\{100\}$ & $\{110\}$ & $\{111\}$ \\
\hline Surface energy ${ }^{a}\left(\mathrm{~J} / \mathrm{m}^{2}\right)$ & 1.99 & 1.41 & 1.15 \\
\hline Modulus of elasticity ${ }^{b}\left(\times 10^{11} \mathrm{~Pa}\right)$ & 1.40 & 2.13 & 2.46 \\
\hline Fracture toughness ${ }^{c}\left(\mathrm{MPa} \mathrm{m}^{1 / 2}\right)$ & 0.95 & 0.90 & 0.82 \\
\hline
\end{tabular}

nitrogen and placed into the glass reaction cell. Using an Eppendorf $300 \mu \mathrm{L}$ pipette, milliQ water was poured in the reaction cell containing the substrate. The water temperature inside the reaction chamber was measured and a rubber ring and glass slide were placed on the reaction chamber. The Peltier-element was set at $0.80 \mathrm{~V}$ to keep the temperature constant at $23^{\circ} \mathrm{C}$.

The substrates were exposed to US for different time intervals ranging from 5 to $60 \mathrm{~min}$ in different rounds. The final exposure time achieved was $180 \mathrm{~min}$. At the end of each experiment, the substrate was removed and the reaction cell was dried with nitrogen. This procedure was repeated for every measurement.

An oxygen plasma cleaner barrel Tepla 300E was used to remove carbonaceous contamination.

\subsubsection{Fractography, imaging and processing}

The silicon substrate surfaces were imaged using SEM before and after erosion took place, also termed fractography. The surface topology of each surface provides information on the damage initiation sites and the possible failure mechanisms. The fractography in our study was done with a High Resolution SEM Zeiss 1550 under a vacuum of $5 \times 10^{-7} \mathrm{~Pa}$. The substrates were imaged within the region of the two micromachined pits where cavitation bubbles were present. The images were made perpendicular to the specimen surface with electron energies of $5 \mathrm{keV}$ and $100 \mathrm{keV}$ with a resolution of $1023 \times 767$ pixels. We did not follow the growth of individual erosion pits in time, since each SEM analysis was performed at a magnification large enough to provide good resolution, but no complete coverage was possible. The scans were always done in the same region, but after the plasma cleaning step needed to remove carbonaceous contamination, it is virtually impossible to find exactly the same erosion pit.

Using the Image Processing Toolbox of MATLAB R2011A, an in-house developed script was used to post-process the SEM images. IMAGEJ processing software was used to perform filtering and noise reduction. For each SEM image, the erosion pit boundaries and areas were determined. The total eroded area $\Delta A$ was registered as well as the total area processed $A$. The erosion pit depth was estimated for the (100) Si substrates using trigonometry; for an erosion pit having an isosceles triangle 
cross-section shape, the depth $(d)$ was calculated with $d=\frac{w}{2} \times \tan \left(54.7^{\circ}\right) ; w$ being the smallest side of the erosion pit used as the base width.

\subsubsection{Sonication using an ultrasonic horn at $20 \mathrm{kHz}$}

A Branson 250 Sonifier (Danbury CT, USA) of the horn type with a microtip of diameter $3 \mathrm{~mm}$ was used to compare the effects of cavitation bubbles generated with a conventional ultrasonic device. The frequency of such a device is $20 \mathrm{kHz}$ and the power provided to the tip is limited by the hardware manufacturer to $70 \%$ of the nominal power of the power supply $(200 \mathrm{~W})$.

Polished p-type Silicon chips with crystallographic orientation (100), (110) and (111) parallel to the surface were taped to the bottom of a beaker filled with $500 \mathrm{~mL}$ of water and placed on an adjustable stage. The ultrasonic horn was set with a ring stand, and the horn tip was placed perpendicular to the silicon substrate at $0.5 \mathrm{~mm}$. The substrates were exposed to cavitation for 5, 10 and $15 \mathrm{~min}$. These irradiation times and conditions are supposed to fall within the incubation-acceleration period [18].

\subsection{Results}

\subsubsection{Cavitation bubbles: observations and simulation results}

The nucleation of bubbles from stabilized bubbles on micromachined pits on silicon surfaces has been described in detail elsewhere [27, 28, 42]. In short, and observed in the ultra-high speed recordings (supplementary movie available), the bubble stabilized on the pit oscillates and sheds off micron sized bubbles with an average radius and number that depends on the power applied (pressure amplitude). The conditions of the experiment described here are similar to the case of one pit and high power setting described previously, and account for a most probable bubble radius of $2 \mu \mathrm{m}$, average equivalent bubble radius of $8 \mu \mathrm{m}$ and 50 bubbles per acoustic cycle. Figure 8.2 shows a top-view example of cavitation around two pits.

The nucleation of bubbles can be sustained for several hours as long as the conditions are kept constant (avoiding liquid evaporation, constant temperature, etc.) by virtue of a phenomenon similar to rectified diffusion [28]. The nucleated bubbles are observed to break up seeding the liquid with their fragments; a cycle of expansion, collapse, break-up and again expansion. Since the driving ultrasound amplitude was high enough to obtain large and deforming bubbles, they tended to collapse against the surface producing impinging liquid jets. See Figure 8.2 for a side view.

For the BI simulations, the typical distance of a bubble's center to the wall is estimated from high-speed side-view recordings as reported in previous works [28] and is taken to be $25 \mu \mathrm{m}$. The absolute driving pressure obtained for that study was 


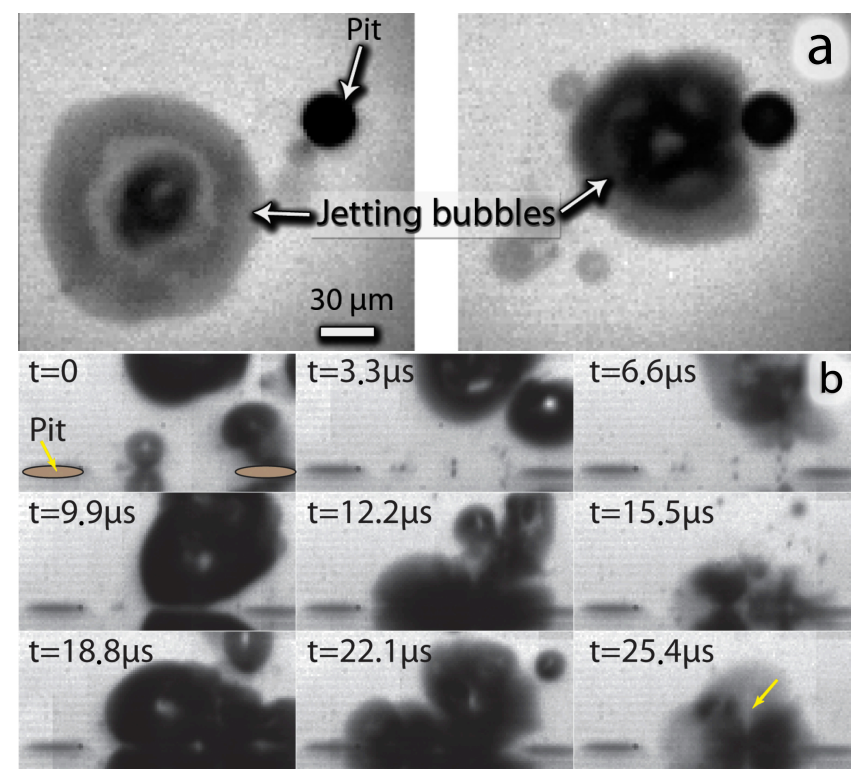

Figure 8.2: (a) Top view of bubbles cavitation close to the silicon surface. The pit has a diameter of $30 \mu \mathrm{m}$. These are individual snapshots taken at $100 \mathrm{kfps}$ and $0.1 \mu \mathrm{s}$ exposure time. (b) Side view of bubbles cavitation close to the silicon surface after being ejected from two pits of $30 \mu \mathrm{m}$ diameter at a distance of $100 \mu \mathrm{m}$. In $t=0$ a bubble ejects from the pit to the right. Subsequent collapses, coalescence and jetting towards the surface (as well as among bubbles) can be seen in all frames, particularly the arrow at $t=25.4 \mu \mathrm{s}$. There are two images per each acoustic cycle.

$130 \mathrm{kPa}$, superimposed on the ambient pressure of 1 bar. These values are used in the BI code, which results in a collapse as shown in Figure 8.3. The final moments simulated in the BI code, for a single bubble of radius $2 \mu \mathrm{m}$, predicted a collapse time from maximum radius to zero of approximately $0.1 \mu \mathrm{s}$ (Figure 8.3). The final velocity of the bubble wall at the point where the BI code stopped was of the order of $10^{2} \mathrm{~m} / \mathrm{s}$. The associated water hammer pressure was calculated to be of the order of 0.1-1 GPa; the shear stress was of the order of 0.1-1 MPa.

As a general trend, smaller bubbles (in the simulated range of $2-25 \mu \mathrm{m}$ in radius) resulted in higher collapse velocities. A larger initial distance $\gamma$ of the bubble from the wall also resulted in higher collapse velocities, with the limit at large $\gamma$ approaching the non-wall-bound spherical bubble collapse velocity.

The collapse evolution calculated by the BI code for a single bubble of radius $15 \mu \mathrm{m}$ near a wall was compared to that of a similar bubble of similar size in the ultra high-speed recording. The collapse time of the bubbles in experiment was of the order of $1 \mu \mathrm{s}$, which is a similar collapse time as predicted by the BI code. More details can be found in the Appendix 8.A 


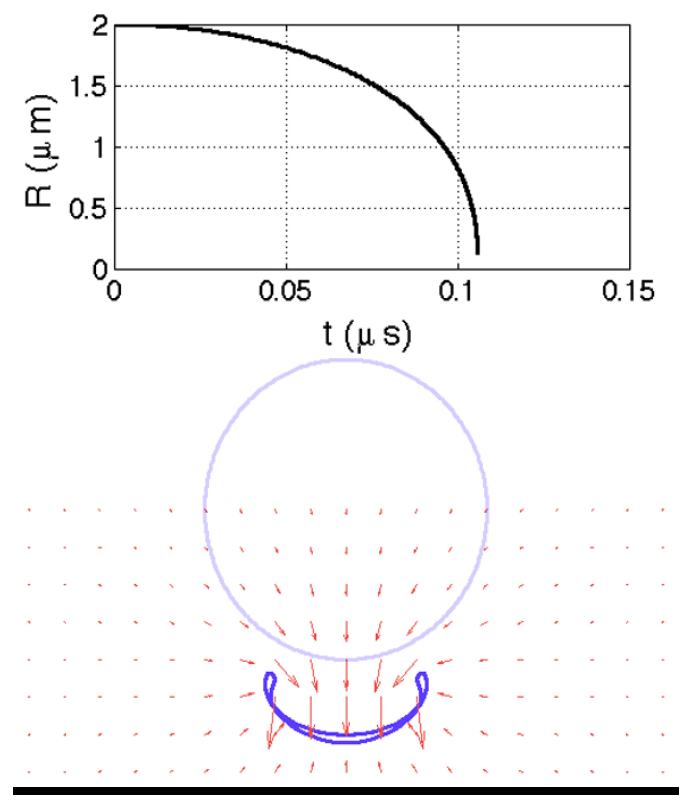

Figure 8.3: Radius-time curve (top) and vector field (bottom; blue is the bubble contour, red are the fluid velocity vectors) as calculated by the BI code for a $2 \mu \mathrm{m}$ initial radius bubble at $4 \mu \mathrm{m}$ from the wall.

The presence of shock wave emissions can be seen in the short exposure photos given in Figure 8.4. This observation was feasible since the refractive index gradient induced by the shock front in the liquid refracts the illumination light. The shock wave width could not be measured properly with the available conditions, nor its propagation speed. The width of the shock wave image should not be mistaken for the shock wave width [34].

\subsubsection{Erosion observations of different crystallographic surfaces of sili- con}

A perspective inclined view for the case of (100) Si can be seen in Figure 8.5 after cavitation exposure for $180 \mathrm{~min}$. Each SEM image showed features depending on the crystallographic orientation; how they form and evolve in time is related to the crystal lattice properties (see Table 8.1). For all orientations we can describe two distinctive types of erosion effects. One is "erosion pitting" and the second is "fracture lines".

If one compares Figure 8.1 and advanced erosion stages as in Figure 8.6, it is possible to observe that for (100) $\mathrm{Si}$, the superficial fracture lines are perpendicular, for (110) the lines have an angle of approximately $54.3^{\circ}$ in between and for (111) an angle of $60^{\circ}$. 

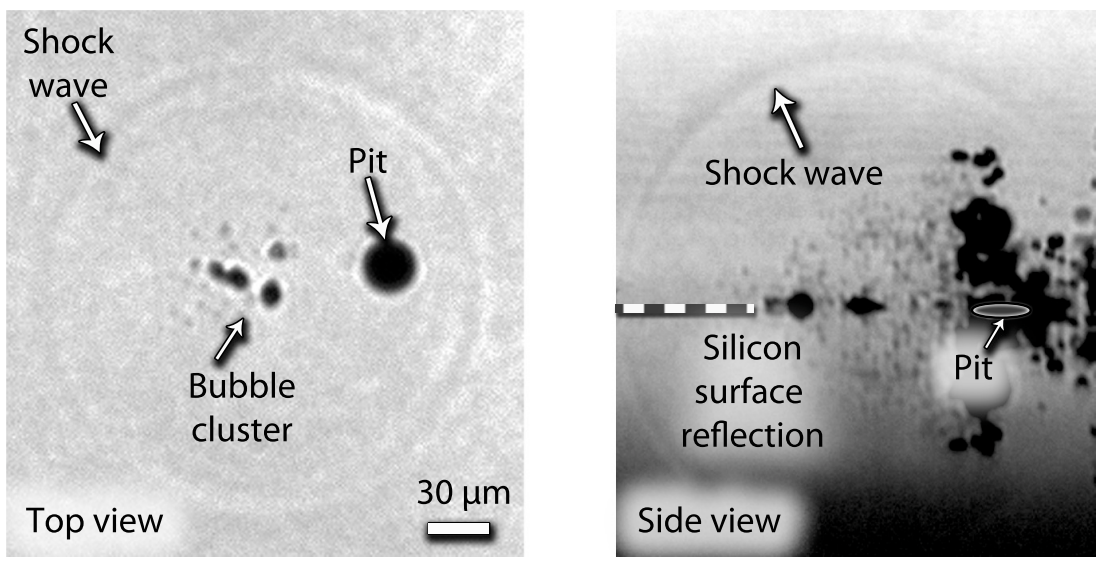

Figure 8.4: Top and side view of bubbles cavitation close to the silicon surface with the emission of a shock wave originated from the bubble clusters. A dashed line indicates the position of the silicon surface.

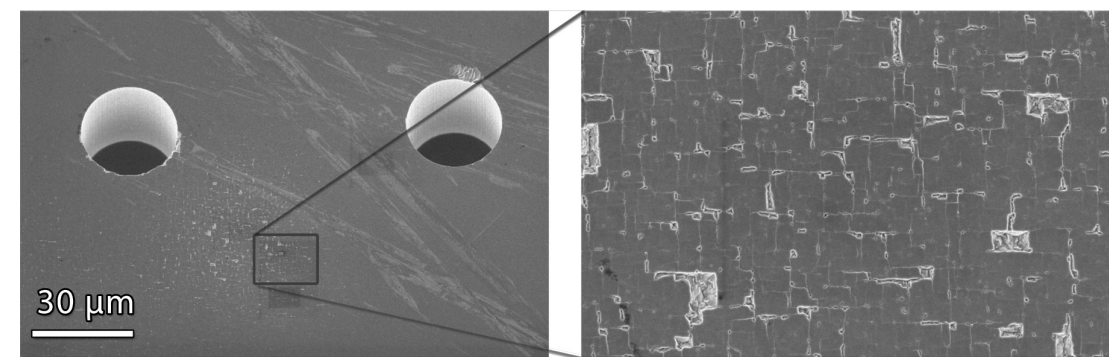

Figure 8.5: An inclined view of a SEM image showing the eroded area in the vicinity of the two pits in a (100) substrate. A zoomed in view to the right side shows the typical erosion features.

\section{Orientation (100)}

A three-dimensional schematic view is provided in Figure 8.7 and compared with SEM images after various times of ultrasound exposure of (100) Si. The red lines represent needle-like cracks which result in grain detachment starting after $50 \mathrm{~min}$ utes. The grains of material detached resemble in most cases a pyramidal shape, as can be seen in detail after $180 \mathrm{~min}$ (formed by the $\{111\}$ planes).

It was not possible to obtain a cross-sectional analysis of the surfaces. Nevertheless, close to the verge of the pit's edge and SEM tilted images (see Fig. 8.5, it is possible to gain some understanding of how the crack lines connect underneath the flat top (100) surface, as depicted in Figure 8.8. From these detailed figures we can understand that the erosion process is not restricted to superficial damage, but also can have effects in the bulk. 
(100)

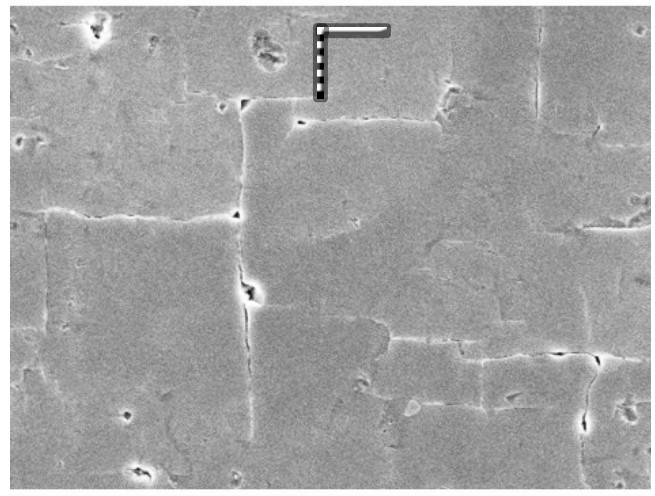

(110)

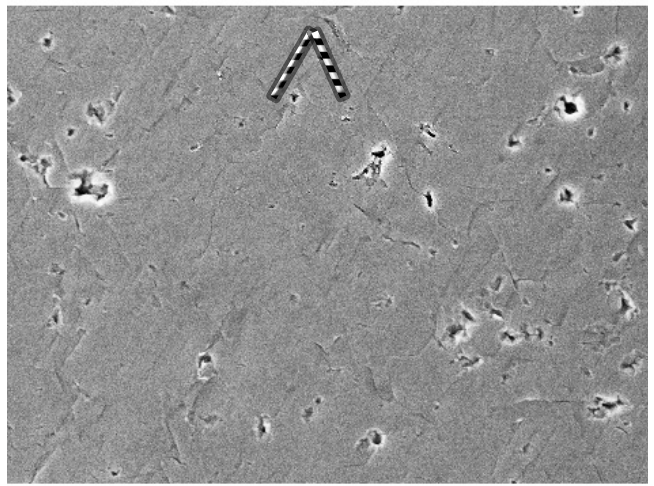

(111)

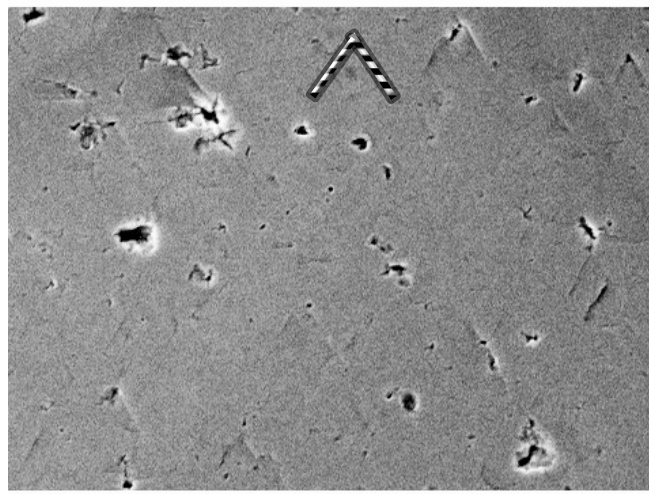

$1 \mu \mathrm{m}$

Figure 8.6: Comparison of the observed advanced erosion fracture lines for (100), (110) and (111) silicon after 180 min of cavitation exposure. The drawn bars indicate the directions followed by the majority of cracks and correspond approximately to Figure 8.1. For (100) these lines are perpendicular, for (110) a combination of $\langle 110\rangle$ and $\langle 112\rangle$ directions complying with (111) cleavage planes at $54.3^{\circ}$ is observed, and for (111) the lines have an angle of $60^{\circ}$ and directions $<110>$ 

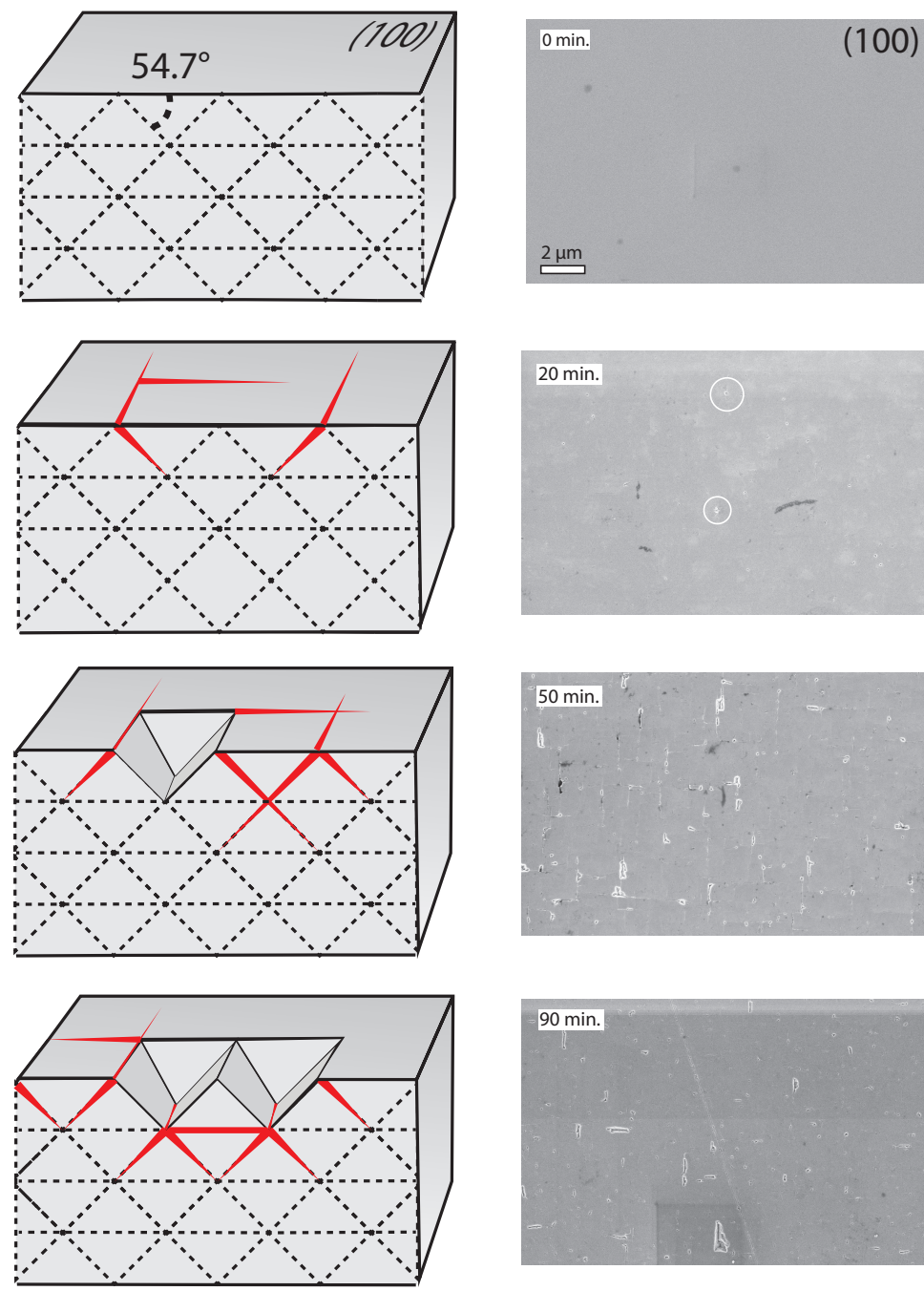

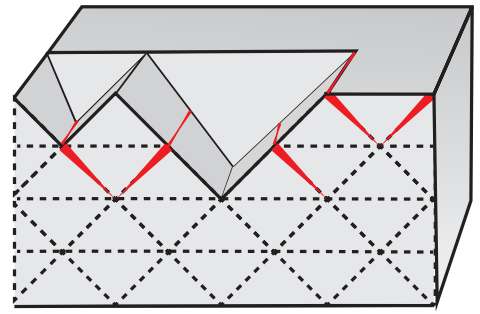

(a)

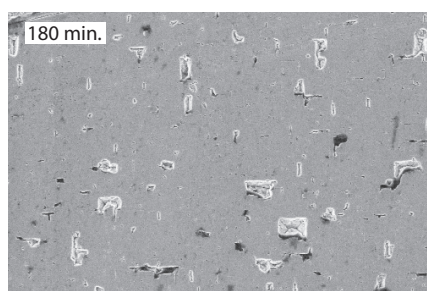

(b)

Figure 8.7: Comparison of an artistic description of the erosion characteristics (a) with the erosion progression in (100) Si wafer (b). The shape of the erosion pits at 180 min shows a pyramidal shape in agreement with the orientations of $\{111\}$ planes. The white circles at 20 min. mark the small cracks observed at early stages. 


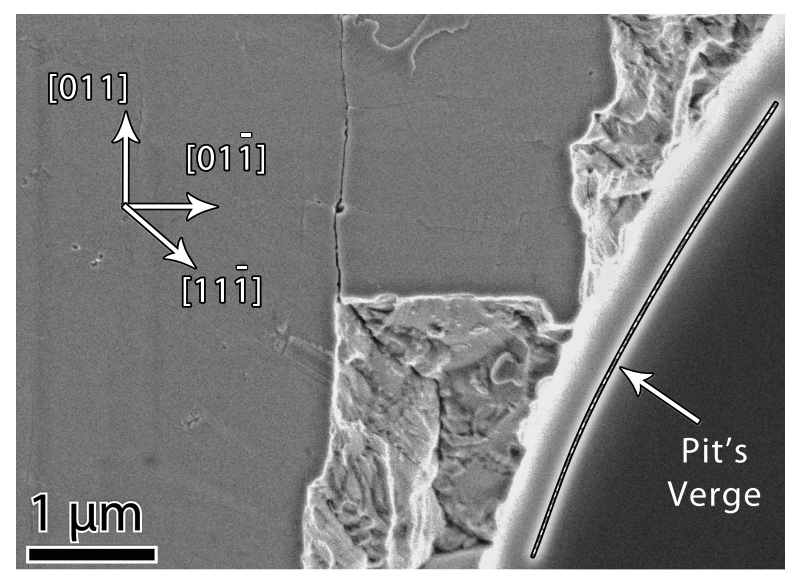

Figure 8.8: Deep cracks at the verge of the pit along $\{111\}$ planes in (100) silicon near a micromachined pit, after 50 minutes irradiation.

All the $\{111\}$ planes are inclined with respect to the (100) surface (top surface of the Si substrate). The surface energy and fracture toughness of $\{111\}$ planes are the lowest, making it energy-wise more probable to create a new surface than in other crystallographic planes (see Table 8.1). The intersecting lines make a $90^{\circ}$ angle.

A certain regularity was observed but not quantified in the distance between the cleavage lines, which got smaller with time. It is recommended for future studies to quantify the location and spread of these lines over time across the whole surface.

\section{Orientation (110)}

Erosion pits and crack lines show different features on (110) compared to (100) Si at the early stages. Some details can be observed in Figure 8.9 .

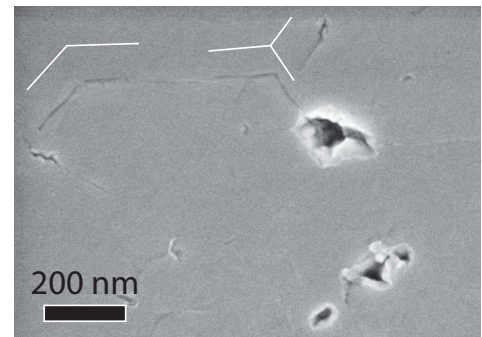

(a)

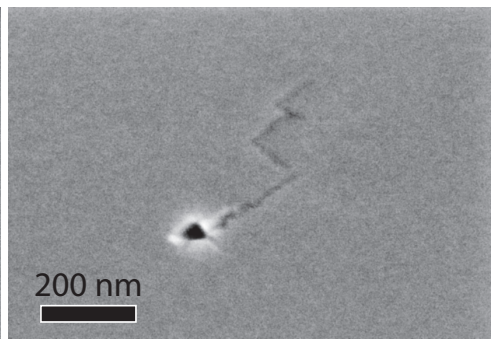

(b)

Figure 8.9: Details of erosion in (110) Si wafer in the early stages (less than $100 \mathrm{~min}$ ). Observe the presence of erosion pits and some cracks having particular shapes: a) lines close to the erosion pits of a length of $200 \mathrm{~nm}$ and b) zig zag cracks with orientations in the [11̄2], [1̄12] and [1̄10] directions. 
There are two types of $\{111\}$ intersecting the (110) surface of the substrate: those that intersect the (110) along [110] are inclined with respect to (110) and those that intersect the (110) along [112] or [112] that are oriented perpendicular to (110).

Based on our observations, the most probable places to develop cracks are at the intersections of $[\overline{1} 10]$ and one of the other two intersection locations. The intersecting lines make an angle of $54.3^{\circ}, 71.4^{\circ}$ and $125.7^{\circ}$ on the (110) surface.

The most striking observation is perhaps the zig-zag crack shown in Figure 8.9p. Such behavior can be understood from literature, as Vicker's indentation tests for the $\{110\}$ indent plane show that the crack "intended" to propagate on the $\{1 \overline{1} 0\}$ planes zig-zags mainly on the $\{1 \overline{1} 1\}$ planes [44, 52].

\section{Orientation (111)}

In the case of (111), the erosion pits and crack lines show a clear difference compared to the two other crystal orientations at the early stages shown in Figure 8.10. The most salient is that crack lines (sometimes six) can intersect and merge in a given point.

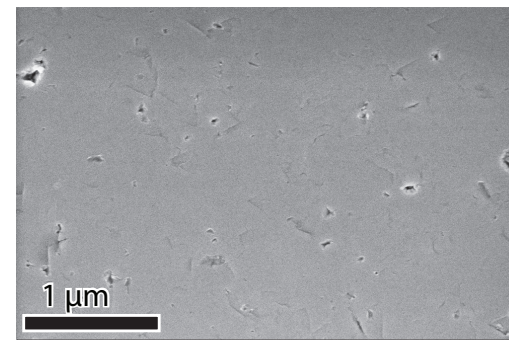

(a)

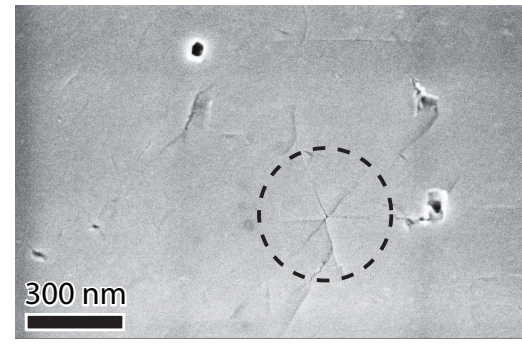

(b)

Figure 8.10: Details of erosion in (111) Si wafer at early stages of erosion (less than 100 min). a) Small crack lines connect in small cornered grooves and small erosion pits scattered on the surface. b) Six crack lines intersect and merge in a given point highlighted by a dashed circle.

There are three $\{111\}$ types of planes intersecting the (111) and as will be shown later, these are all likely to grow cracks. The intersecting lines make an angle of $60^{\circ}$ on the (111) surface in agreement with the situation depicted in Figure 8.10

\subsubsection{Erosion by an ultrasonic horn}

Exposure to the ultrasonic horn also resulted in erosion of the silicon surfaces (100) and (110) (Fig. 8.11), but the eroded parts were not as concentrated as in the micro-pit experiments. The erosion pits look larger for the same sonication period. Evidently the effect of a lower frequency has a strong influence on the mechanical damage when compared to higher frequencies as employed for the micropits setup. For (111) there 
was no damage that could be imaged at least after $10 \mathrm{~min}$. Longer exposure time

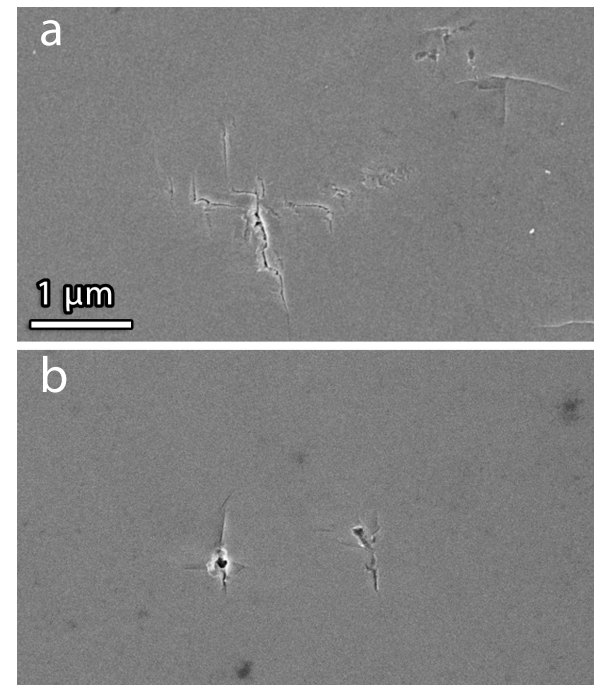

Figure 8.11: Details of the erosion in (100) Si wafer (a) and (110) (b) at early stages of erosion (10 min expossure at $20 \mathrm{kHz}$.)

experiments were not conducted as it was reported that for $20 \mathrm{kHz}$ and a horn cavitation system the incubation period is larger (four hours) [18]. This can be attributed to the differences in the dynamic loading and physico-chemical conditions given by our setup compared to the horn.

\subsection{Discussion}

\subsubsection{Study of Si fatigue}

Even though erosion and fatigue studies in silicon are available in the literature, a one-to-one comparison with our results cannot be given (different types of loading, frequency, test duration). For that reason we consider it important to try to provide some reference values and discussions on this subject.

Each collapsing bubble that was produced by the micropit ultrasound device results in a force load on the silicon surface (either a liquid jet or shockwave). Each load by itself may not be enough to fracture the silicon, but repeated load can lead to fatigue failure. Figure 3 of Varvani's work [52] shows an S/N curve (stress vs. number of loads), without an empirical fitting factor [52, 57, 72]. Using this curve, we can estimate the number of bubbles (loads, $N$ ) needed to make the material fail. The BI method for single bubble collapse near a wall predicted a water hammer pressure ranging from 0.1 to $1 \mathrm{GPa}$, which corresponds to a number of loads $(\mathrm{N})$ larger than 
$10^{8}$. Using the estimated number of bubble impacts as described in Section 8.3.1. along $50 \mathrm{~min}$, when clear damage to the surface is observed, leads to a number of impacts in the order of $10^{6}$. If we consider all these impacts to be loads on a single point, Si would fail if each impact would be of $\sim 2.4 \mathrm{GPa}$.

From these values we can only say that the order of magnitudes of the water hammer jets the BI code provides are relatively close to the expected stresses known to make Si fail by virtue of fatigue effects. However, the contribution of shock waves cannot be ruled out.

The pressure exerted on an AFM tip by bubble clusters similar to the ones given here was estimated to be $\sim 5 \mathrm{MPa}$ [10]. This should be taken as a lower estimate since the distance from the silicon substrate from which the bubbles were generated to the AFM tip was ca. $100 \mu \mathrm{m}$, instead of collapsing near the silicon surface as this study has covered.

When comparing the stresses provided by shock waves reported in literature (10 GPa [34]), with the $S / N$ curves of Varvani's methodology [52] it is not possible to get a value of loads $N$, because the lowest amount of cycles accounted were $\sim 200$, corresponding to roughly $2.8 \mathrm{GPa}$ stress value. If the shock waves impacting the Si surfaces in this study would exert a $10 \mathrm{GPa}$ stress value, that would certainly make Si fail from the first impact; but in reality the zone of impact is embedded in a larger material matrix, making it a more complex situation than a free-standing piece used in other fatigue experiments. We can speculate that the type of bubble collapse encountered in our experiments is different than for the conditions at which those high pressure values were measured; the most important being that we have a bubble cluster collapse, and in those cases, bubbles experience a "shielding effect" that may lead to reduced shock wave pressures [42, 73]. Interestingly, the metallization of c-Si with shockwaves has been described [74].

From the results of Virot and coworkers [18] on Si cavitation erosion, a better resistance to cavitation erosion of $\mathrm{Si}$ was found when compared to glass, having long incubation times ( $\sim 4$ hours). In general, even though pits and cracks were formed, the final erosion state differed from other materials, like glasses or metals. The superhydrophilicity of the surface after sonication was explained as a result of increased density of hydroxyl groups at the newly created surfaces or perhaps an inverse lotus effect. Shifts in the Raman spectra pointed at anisotropic compressive stresses along with lattice deformation; deconvolution analysis gave evidence of the presence of grain boundaries or crystalline $\mathrm{Si}$ particles, the coexistence of crystalline $\mathrm{Si}$ (c-Si), and amorphous $\mathrm{Si}(\mathrm{a}-\mathrm{Si})$. The latter was correlated with plastically deformed areas observed with SEM, and may result from a loss of lattice stability during the cyclic compressions/decompressions of the collapsing bubbles. TEM analysis coincided with SEM results, and additionally revealed a more complex highly stressed and distorted structure, showing several possible phases: c-Si, a-Si, and polycrystalline $\mathrm{Si}$ 
(poly-Si). It was also concluded that local changes in $\mathrm{Si}$, like amorphization and metalization, could fracture, deform, and dislocate the Si sub-surface. This could in turn change its density leading to volume expansion and contraction affecting the electron density of states (thermal and electrical properties of $\mathrm{Si}$ ) promoting the emission of $\mathrm{Ar}^{*}$ by mechanoluminescence. Contrary to other studies found in literature, no aging effect of Si in pure water for several days or in parts not directly exposed to US was found.

To complete our study further experiments might be conducted in the future to measure the effect of shock waves and surface erosion evolution. All these phenomena are certainly interconnected and may have a synergistic effect towards erosion of Si surfaces.

The ultimate cause for fatigue of $\mathrm{Si}$, as a brittle material, would be the damage accumulation (formation and propagation of cracks) as a result of small stress loads (either jet or shockwave impact), aggravated by the chemical corrosion effects of the products of sonochemical products of bubble cavitation. Wave propagations as a result of the pressure pulses against the Si surface may contribute to the complex erosion scenario.

\subsubsection{Area and volume erosion}

The eroded volumes calculated from SEM images are shown in Figure 8.12, The growth and interconnection (coarsening) of small erosion pits creates deep craterlike erosion pits. The amount of smaller erosion pits increases with time (type a) and other larger erosion pits are formed by coalescence (b and c). For instance, the amount of erosion pits with depths 100-200 nm (black line) shows a decline after 50 $\mathrm{min}$ and at the same time, deeper erosion pits $(>500 \mathrm{~nm})$ increase in number. This can be understood as a cascading effect that indicates what seems to be the end of the incubation period.

The erosion features for (110) and (111) were completely different. Smaller in number and eroded area and no clear geometrical figure could be related to the shape of the erosion pits. Hence, a similar assessment as for (100) was not possible.

Comparing the number of erosion pits and eroded area calculated in Section 8.2.5. (100) clearly shows a qualitatively different progression in the erosion process compared with (110) and (111). In all three cases the initial stage seems to be consistent with the presence of small cracks (small openings on the surface) that upon the action of the collapse of cavitation bubbles, start to grow. As the cavitation exposure progresses, these cracks grow and interconnect; (100) show a more pronounced presence of superficial cracks. These cracks can reach deep into the crystal bulk (as seen in Fig. 8.8) and eventually, while the erosion pits also grow in area or volume, the "weaker" planes start to intersect (in a pyramidal fashion matching the $\{111\}$ planes) making it possible for a grain or piece of material to detach. The large detached ma- 


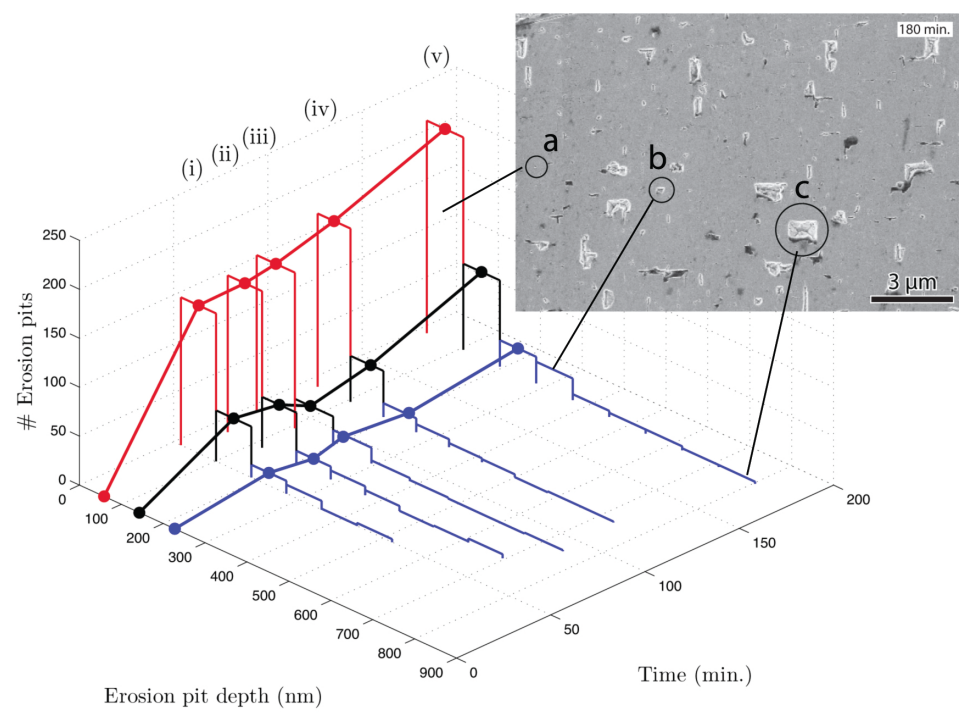

Figure 8.12: Histogram plots of the calculated erosion pit depth in (100) silicon as a function of the number of erosion pits and the irradiation time: (i) 50 min., (ii) 70 min., (iii) 90 min., (iv) $120 \mathrm{~min}$ and (v) $180 \mathrm{~min}$. The first three histogram bins are colored and connected to show their particular evolution. The image resolution did not allow pit depth extraction for irradiation times below 50 minutes.

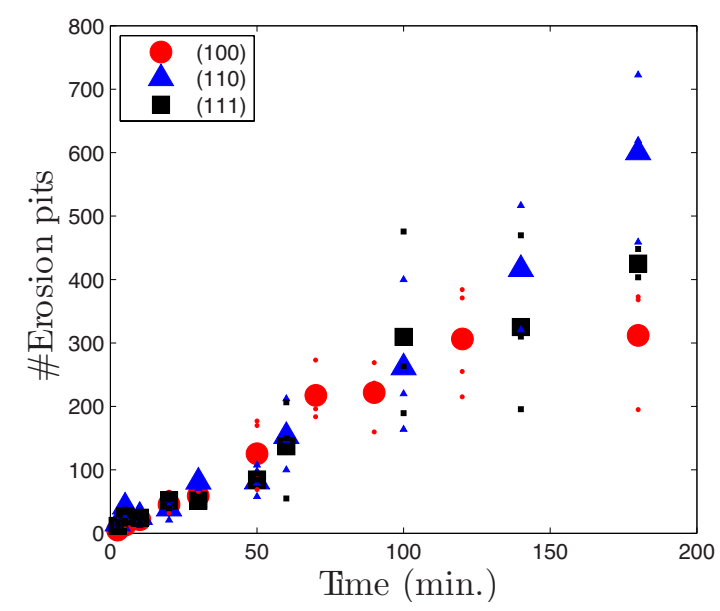

Figure 8.13: Absolute number of erosion pits as a function of the sonication exposure time. The scattered points are individual substrate measurements and the bold symbol represents the averaged value for that specific sonication time. 
terial (which presumably could have contained at least four erosion pits) forms a new and larger pit, reducing the total number $N$ of erosion pits (see Fig. 8.13).

For (110) and (111) the situation seems different. We see that the way the cracks and planes intersect, indeed allows for the formation of erosion pits, but not in a pyramidal shape as cavitation exposure increases (which is the case for (100)). Interestingly, the existing pits do not interconnect in the same fashion, giving an increase in the number of pits, but not an increase in the area (crack propagation is impeded), as is the case for (100) (see Fig. 8.14).

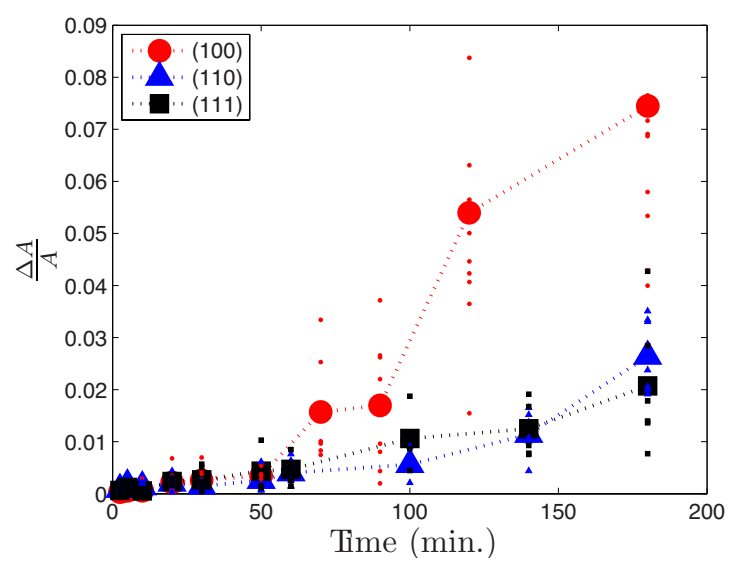

Figure 8.14: Non-dimensional eroded area $\left(\frac{\Delta A}{A}\right)$ plotted as a function of sonication exposure time. The scattered points are individual measurements and the bold symbol represents the averaged value for that specific sonication time.

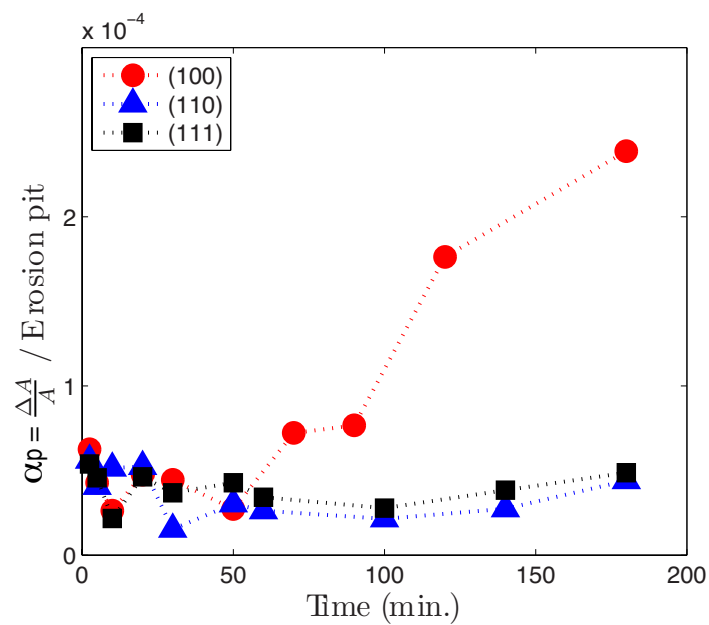

Figure 8.15: Plot of $\alpha_{p}$ (non-dimensional eroded area divided by the number of erosion pits) as a function of sonication exposure time. 
Plotting the non-dimensional eroded area divided by the number of erosion pits, which we define as $\alpha_{p}=\frac{\Delta A}{A} / N$, gives insight into the coarsening progression, i.e. interconnection of the erosion pits. Fig. 8.15 clearly shows the differences among (100) compared with (110) and (111), where these last two show a similar and relatively horizontal slope, whereas after the incubation time (50 min), (100) increases with a higher slope. Note that we define the incubation time as the time in which all the analyzed parameters show clear differences for each silicon crystallographic configuration. Other authors use line extrapolation from their erosion rate plots in the acceleration stage [50], but we are not using this approach because the time span and trends we observe are not conclusive to know which period of erosion has been reached. Other workers reported a longer resistance to erosion [18], which from our horn cavitation preliminary measurements appears to be the case for lower frequencies and a similar cavitation system as shown in Fig. 8.11.

When considering the physical properties of the materials studied here (see Table 8.1 , we can see that the modulus of elasticity for $\{100\}$ is $1.5 \times$ and $1.8 \times$ smaller than $\{110\}$ and $\{111\}$ respectively. The ability of absorbing forcing loads (either liquid jets or shockwaves) seems to be related to the fact that (100) erodes faster. In literature it is reported that Vicker's indentation performed on (100) surfaces, shows higher hardness (resistance to permanent deformation) and lower toughness (ability to absorb energy and deform in a plastic fashion without fracturing) for (100) [44]. This can explain why (100) shows more fragile behavior. But it must also be noted that the dynamic type of loading of cavitation is faster than indentation tests, which can certainly have an influence on the type of erosion [18].

According to literature, the fracture toughness is approximately the same for the three crystal orientations. This might be connected to the fact that the presence of pre-existing cracks, and the appearance of new cracks, follow a similar trend in all cases (Fig. 8.13).

The marked difference of (100) having extended crack lines and detachment of small grains, not observed in the other silicon substrates with different crystallographic orientation, needs further discussion. Based on our observations it is assumed that damage formation on $\{111\}$ is related to shear forces acting on these planes as a result of jetting, shockwaves or any other loading of the Si surface. If the damaging force acts perpendicular to the Si single crystal surface, the resulting maximum shear stress on $\{111\}$ is proportional to $\sin 2 \phi$. This expression can be derived from the Schmid factor $m$ that relates the applied stress $\sigma$, to the resolved shear stress $\tau$ on a slip plane, for a dislocation moving in a certain slip direction: $\tau=m \sigma$. It is defined as $m=\cos (\phi) \cos (\lambda)$, with $\phi$ the angle between the applied load and the slip plane normal and $\lambda$ the angle between the applied load and the slip direction. Since the exact cause of damage formation on $\{111\}$ is not completely clear and might not be directly related to dislocation movement, an indicative value of the Schmid factor 
can be obtained assuming damage to occur in the direction of maximum shear stress. This direction is obtained by decomposing the applied force unit vector in a component perpendicular to and parallel with the $\{111\}$ plane. The first component is in the direction of the slip plane normal and defines the normal stress on $\{111\}$. It is proportional to $\cos \phi$ which is also equal to the first term of the Schmid factor. The second component is parallel to the slip plane and defines the shear stress on $\{111\}$. It is proportional to $\sin \phi$ and constitutes the second term of the Schmid factor. The product of both terms leads to the $\sin 2 \phi$ dependence. It can be concluded that damage formation on $\{111\}$ planes for $\mathrm{Si}(100)$ is most probable, since the corresponding value of $\sin 2 \phi$ is largest. Experimental evidence, such as shown in Fig. 8.14, supports this idea. Furthermore, Fig. 8.15 shows that after some incubation/nucleation time rapid growth of the eroded area of an erosion pit occurs. The collective damage formation on all $\{111\}$ planes at a (100) surface results in pyramidal shaped grains that can break off consecutively. In this way cracks can grow out easily from an erosion pit to form fracture lines. For Si (110) such a mechanism is less likely, as under the action of the damaging force perpendicular to the (110) surface, only two $\{111\}$ are active, i.e. $\sin 2 \phi>0$. For grains to break off more than two active $\{111\}$ are required; four for (100) as can be seen in Fig. 8.7. For Si (111), three $\{111\}$ are active, but the value of $\sin 2 \phi$ and the number of active $\{111\}$ is smaller than for $\mathrm{Si}$ (100), making damage growth slower. It would be interesting to extend the sonication exposure time to see the development of $\alpha_{p}$ in time for $\mathrm{Si}$ (110) and $\mathrm{Si}$ (111).

Following the same methodologies as reported by Virot et al. [18] using TEM, micro-Raman and X-ray diffraction would provide more useful information on the changes due to sonication occurring in all crystallographic Si substrates.

\subsubsection{Ultrasound erosion systems}

To illustrate the advantages of using the device with micromachined pits over conventional testing apparatuses we compared it with the horn experiments conducted. First, the amount of water used is $10^{3}$ larger which, depending on how many experimental tests, or the purity of water, or other liquid used, can result in a significant economical cost. Second, as the area exposed to the horn cavitation conditions is considerably larger, it was harder to find the erosion features. The lower frequency apparently influenced the erosion of (111) substrates for which no damage could be found for the studied times. Furthermore, the conditions to hold the horn and the substrates in place were more difficult to control, hence in a more complex experimental measurement strategy, reproducibility would be affected. Additionally, it is not straightforward to image the events of bubble cavitation in the small gap between the horn and the exposed substrate. For complimentary information on a horn system used to study the (100) erosion and other phenomena like mechanoluminescence and phase changes on silicon structure (amorphization), we refer the reader to the existing 
literature [18].

\subsection{Conclusions}

With the setup described in this work, we demonstrate its advantages to study cavitation in well controlled conditions. A detailed study can be performed on the erosion at the microscale during the incubation period. Several parameters such as frequency, pressure amplitude, gas content and liquids can be varied to address the cavitation erosion effects of bubbles on several types of materials, besides silicon. It also offers advantages over conventional experimental setups like sonicators (baths or horns), hydrodynamic flows or jets cavitation erosion test, in the sense that the current setup uses smaller volumes, allows the direct recording of the phenomena involved, and the location of cavitation bubbles can be better controlled. The possibility to study cavitation erosion, right from the initial incubation period and through into more advanced stages (180 min), has been demonstrated in $\mathrm{Si}$ with the help of micromachined pits.

We observed that the concerted effect of various sources of damage formation such as jetting, shock waves, direct bubble impact and surface stress corrosion can all cause the damage observed for the three crystallographic silicon surfaces studied, although each of the three surfaces have a different resistance to erosion. For (100) silicon, the incubation time was of the order of $50 \mathrm{~min}$, whereas for (110) and (111) apparently the incubation period is larger than the total 180 min sonication. A possible explanation for this occurrence has been provided.

The design of an experiment in which each of the cavitation damage mechanisms can be isolated will be a challenge for future research. These studies could be carried out at different powers and other materials with an improved imaging system to quantify better the shock wave emissions.

\section{Acknowledgments}

The authors acknowledge Stefan Schlautmann for his support in the microfabrication processes. The assistance of Mark Smithers with SEM imaging and Guillaume Lajoinie with the Brandaris recordings and analysis is also appreciated. D.F.R. acknowledges the support provided by Dr. Alberto Rolo in fruitful discussions. The authors also thank the inspiring words of Prof. Andrea Prosperetti.

This research was supported by the Technology Foundation STW, Applied Science Division of NWO and the Technology Program of the Ministry of Economic Affairs, The Netherlands under the number 07391. 


\section{References}

[1] K. Suslick, "The chemistry of ultrasound", Encyclopaedia Britannica Yearbook of Science and the Future 1994, 138-155 (1994).

[2] D. Lohse, "Bubble puzzles", Physics Today 56, 36-41 (2003).

[3] L. Rayleigh, "On the pressure developed in a liquid during the collapse of a spherical cavity", Philos. Mag. Series 6 34, 94-98 (1917).

[4] C. Brennen, Cavitation and Bubble Dynamics, Oxford engineering science series (Oxford University Press) (1995).

[5] F. Lugli and F. Zerbetto, "An introduction to bubble dynamics", Phys. Chem. Chem. Phys. 9, 2447-2456 (2007).

[6] T. Graf, H. Fischer, H. Reul, and G. Rau, "Cavitation potential of mechanical heart valve prostheses", Int. J. Artif. Organs 14, 169-174 (1991).

[7] R. Arndt, "Cavitation in fluid machinery and hydraulic structures", Annu. Rev. Fluid Mech. 13, 273-326 (1981).

[8] D. Dowson and C. M. Taylor, "Cavitation in bearings”, Annu. Rev. Fluid Mech. 11, 35-65 (1979).

[9] M. O. Lamminen, H. W. Walker, and L. K. Weavers, "Mechanisms and factors influencing the ultrasonic cleaning of particle-fouled ceramic membranes", Journal of Membrane Science 237, 213-223 (2004).

[10] D. Fernandez Rivas, B. Verhaagen, J. Seddon, A. G. Zijlstra, L. Jiang, L. van der Sluis, M. Versluis, D. Lohse, and H. J. G. E. Gardeniers, "Localized removal of layers of metal, polymer or biomaterial by cavitating microbubbles", Biomicrofluidics Accepted (2012).

[11] M. Virot, T. Chave, S. I. Nikitenko, D. G. Shchukin, T. Zemb, and H. Möhwald, "Acoustic cavitation at the water-glass interface", J. Phys. Chem. C 114, 13083-13091 (2010).

[12] H. S. Chen, Y. J. Li, D. R. Chen, and J. D. Wang, "Experimental and numerical investigations on development of cavitation erosion pits on solid surface", Tribology Letters 26, 153-159 (2007).

[13] T. J. Mason, A. J. Cobley, J. E. Graves, and D. Morgan, "New evidence for the inverse dependence of mechanical and chemical effects on the frequency of ultrasound", Ultrason. Sonochem. 18, 226-230 (2011).

[14] A. El-Bahar, S. Stolyarova, A. Chack, R. Weil, R. Beserman, and Y. Nemirovsky, "Ultrasound treatment for porous silicon photoluminescence enhancement”, Phys. Status Solidi A 197, 340-344 (2003).

[15] E. V. Skorb, D. G. Shchukin, H. Möhwald, and D. V. Andreeva, "Ultrasound-driven design of metal surface nanofoams", Nanoscale 2, 722-722 (2010).

[16] G. G. A. Fatjo, M. Hadfield, and K. Tabeshfar, "Pseudoplastic deformation pits on polished ceramics due to cavitation erosion", Ceram. Int. 37, 1919-1927 (2011).

[17] A. Podolian, A. Nadtochiy, V. Kuryliuk, O. Korotchenkov, J. Schmid, M. Drapalik, and V. Schlosser, "The potential of sonicated water in the cleaning processes of silicon wafers", Sol. Energy Mater. Sol. Cells 95, 765 $-772(2011)$.

[18] M. Virot, R. Pflieger, E. V. Skorb, J. Ravaux, T. Zemb, and H. Möhwald, "Crystalline silicon under acoustic cavitation: From mechanoluminescence to amorphization”, J. Phys. Chem. C 116, 15493-15499 (2012).

[19] J. Koshka, S. Ostapenko, T. Ruf, and J. M. Zhang, "Activation of luminescence in polycrystalline silicon thin films by ultrasound treatment”, Appl. Phys. Lett. 69, 2537-2539 (1996). 
[20] S. Kalem, O. Yavuzcetin, and C. Altineller, "Effect of light exposure and ultrasound on the formation of porous silicon”, Journal of Porous Materials 7, 381-383 (2000).

[21] E. V. Skorb, D. V. Andreeva, and H. Möhwald, "Generation of a porous luminescent structure through ultrasonically induced pathways of silicon modification”, Angew. Chem. Int. Ed. 51, 5138-5142 (2012).

[22] J. Rooze, E. V. Rebrov, J. C. Schouten, and J. T. Keurentjes, "Effect of resonance frequency, power input, and saturation gas type on the oxidation efficiency of an ultrasound horn", Ultrason. Sonochem. 18, 209 - 215 (2011).

[23] E. Zwaan, S. Le Gac, K. Tsuji, and C.-D. Ohl, "Controlled cavitation in microfluidic systems", Phys. Rev. Lett. 98, 254501 (2007).

[24] D. F. Rivas and J. G. E. Gardeniers, "On the resilience of pdms microchannels after violent optical breakdown microbubble cavitation”, ASME Conference Proceedings 2008, 1939-1942 (2008).

[25] S. Howkins, "Solid erosion in low-amplitude sound fields", J. Acoust. Soc. Am. 39, 55 (1966).

[26] N. Bremond, M. Arora, C. D. Ohl, and D. Lohse, "Controlled multi-bubble surface cavitation", Phys. Rev. Lett. 96, 224501 (2006).

[27] D. Fernandez Rivas, A. Prosperetti, A. G. Zijlstra, D. Lohse, and H. J. G. E. Gardeniers, "Efficient sonochemistry through microbubbles generated with micromachined surfaces", Angew. Chem. Int. Ed. 49, 9699-9701 (2010).

[28] D. Fernandez Rivas, L. Stricker, A. G. Zijlstra, H. J. G. E. Gardeniers, D. Lohse, and A. Prosperetti, "U1trasound artificially nucleated bubbles and their sonochemical radical production", Ultrason. Sonochem. Accepted (2012).

[29] T. B. Benjamin and A. T. Ellis, "The collapse of cavitation bubbles and the pressures thereby produced against solid boundaries”, Phil. Trans. R. Soc. Lond. A 260, 221-240 (1966).

[30] A. Philipp and W. Lauterborn, "Cavitation erosion by single laser-produced bubbles", J. Fluid Mech. 361, 75-116 (1998).

[31] M. Dular and A. Osterman, "Pit clustering in cavitation erosion", Wear 265, 811-820 (2008).

[32] C. M. Preece and J. H. Brunton, “A comparison of liquid impact erosion and cavitation erosion”, Wear 60, 269-284 (1980).

[33] E. A. Brujan, G. S. Keen, A. Vogel, and J. R. Blake, "The final stage of the collapse of a cavitation bubble close to a rigid boundary", Phys. Fluids 14, 85-92 (2002).

[34] E. A. Brujan, T. Ikeda, and Y. Matsumoto, "On the pressure of cavitation bubbles", Exp. Therm Fluid Sci. 32 , 1188-1191 (2008).

[35] N. D. Shutler and R. B. Mesler, "A photographic study of dynamics and damage capabilities of bubbles collapsing near solid boundaries”, J. Basic Eng-T ASME 87, 511-\& (1965).

[36] A. Brotchie, F. Grieser, and M. Ashokkumar, "Effect of power and frequency on bubble-size distributions in acoustic cavitation”, Physical Review Letters 102, 84302 (2009).

[37] M. Ashokkumar, J. Lee, Y. Iida, K. Yasui, T. Kozuka, T. Tuziuti, and A. Towata, "Spatial distribution of acoustic cavitation bubbles at different ultrasound frequencies”, ChemPhysChem 11, 1680-1684 (2010).

[38] C. J. B. Vian, P. R. Birkin, and T. C. Leighton, "Cluster collapse in a cylindrical cell: Correlating multibubble sonoluminescence, acoustic pressure, and erosion”, J. Phys. Chem. C 114, 16416-16425 (2010).

[39] S. N. Buravova and Y. A. Gordopolov, "Cavitation erosion as a kind of dynamic damage”, Int. J. Fracture 170, 83-93 (2011). 
[40] N. K. Bourne, "On the collapse of cavities", Shock Waves 11, 447-455 (2002).

[41] C.-D. Ohl, A. Philipp, and W. Lauterborn, "Cavitation bubble collapse studied at 20 million frames per second”, Ann. Phys. 507, 26-34 (1995), URL http://dx.doi.org/10.1002/andp.19955070104

[42] D. Fernandez Rivas, M. Ashokkumar, T. Leong, K. Yasui, T. Tuziuti, S. Kentish, D. Lohse, and H. J. Gardeniers, "Sonoluminescence and sonochemiluminescence from a microreactor", Ultrason. Sonochem. 19, 12521259 (2012).

[43] N. C. Eddingsaas and K. S. Suslick, "Mechanoluminescence: Light from sonication of crystal slurries", Nature 444, 163-163 (2006).

[44] F. Ebrahimi and L. Kalwani, "Fracture anisotropy in silicon single crystal", Materials Science and Engineering a-Structural Materials Properties Microstructure and Processing 268, 116-126 (1999).

[45] N. Maluf, An introduction to microelectromechanical systems engineering (Artech House) (2000).

[46] G. G. A. Fatjo, M. Hadfield, C. Vieillard, and J. Sekulic, "Early stage cavitation erosion within ceramics-an experimental investigation”, Ceram. Int. 35, 3301-3312 (2009).

[47] B. Karunamurthy, M. Hadfield, C. Vieillard, and G. Morales, "Cavitation erosion in silicon nitride: Experimental investigations on the mechanism of material degradation", Tribol. Int. 43, 2251-2257 (2010).

[48] C. G. Stephanis, J. G. Hatiris, and D. E. Mourmouras, "The process (mechanism) of erosion of soluble brittle materials caused by cavitation”, Ultrason. Sonochem. 4, 269-271 (1997).

[49] J.-P. Franc and J.-M. Michel, Fundamentals of Cavitation, Fluid Mechanics and Its Applications (Kluwer Academic Publishers) (2004).

[50] S. Hattori, T. Hirose, and K. Sugiyama, "Prediction method for cavitation erosion based on measurement of bubble collapse impact loads", Wear 269, 507-514 (2010).

[51] C. L. Muhlstein, S. B. Brown, and R. O. Ritchie, "High-cycle fatigue of single-crystal silicon thin films", J. Microelectromech. S. 10, 593-600 (2001).

[52] A. Varvani-Farahani, "Silicon mems components: a fatigue life assessment approach", Microsyst. Technol. 11, 129-134 (2005).

[53] K. Komai, K. Minoshima, and S. Inoue, "Fracture and fatigue behavior of single crystal silicon microelements and nanoscopic afm damage evaluation", Microsystem Technologies 5, 30-37 (1998).

[54] S. M. Wiederhorn, S. W. Freiman, E. R. Fuller, and C. J. Simmons, "Effects of water and other dielectrics on crack growth", Journal of Materials Science 17, 3460-3478 (1982), 10.1007/BF00752191.

[55] T. Okada, Y. Iwai, S. Hattori, and N. Tanimura, "Relation between impact load and the damage produced by cavitation bubble collapse", Wear 184, 231-239 (1995).

[56] J. Steller, "International cavitation erosion test and quantitative assessment of material resistance to cavitation", Wear 233, 51-64 (1999).

[57] A. Varvani-Farahani, "A new energy-critical plane parameter for fatigue life assessment of various metallic materials subjected to in-phase and out-of-phase multiaxial fatigue loading conditions", Int. J. Fatigue 22, 295-305 (2000).

[58] C. Chin, C. Lancée, J. Borsboom, F. Mastik, M. Frijlink, N. de Jong, M. Versluis, and D. Lohse, "Brandaris 128: A digital 25 million frames per second camera with 128 highly sensitive frames", Rev. Sci. Instrum. 74, 5026-5034 (2003).

[59] A. van der Bos, A. Zijlstra, E. Gelderblom, and M. Versluis, "ilif: illumination by laser-induced fluorescence for single flash imaging on a nanoseconds timescale”, Exp. Fluids 51, 1283-1289 (2011). 
[60] J. H. Brunton, "High-speed liquid impact”, Phil. Trans. R. Soc. Lond. A 260, 79-85 (1966).

[61] S. S. Cook, "Erosion by water-hammer", Proceeding of the Royal Society London A 119, 481-488 (1928).

[62] C.-D. Ohl, M. Arora, R. Ikink, N. De Jong, M. Versluis, M. Delius, and D. Lohse, "Sonoporation from jetting cavitation bubbles", Biophys. J. 91, 4285-4295 (2006).

[63] M. Brede and P. Haasen, "The brittle-to-ductile transition in doped silicon as a model substance", Acta Metall 36, 2003-2018 (1988).

[64] G. Michot and A. George, "Fracture and crack tip plasticity in silicon and gallium-arsenide", in Structure and Properties of Dislocations, edited by S. G. Roberts, D. B. Holt, and P. R. Wilshaw, 104, 385-396 (1989).

[65] A. George and G. Michot, "Dislocation loops at crack tips: nucleation and growth - an experimental study in silicon”, Mater. Sci. Eng., A 164, 118-134 (1993).

[66] P. B. Hirsch, S. G. Roberts, and J. Samuels, "The brittle-ductile transition in silicon. ii interpretation", Proc. R. Soc. London, Ser. A 421, 25-53 (1989).

[67] C. S. John, "The brittle-to-ductile transition in pre-cleaved silicon single crystals", Philos. Mag. 32, 1193-1212 (1975).

[68] M. Tanaka, K. Higashida, H. Nakashima, H. Takagi, and M. Fujiwara, "Orientation dependence of fracture toughness measured by indentation methods and its relation to surface energy in single crystal silicon", Int. J. Fracture 139, 383-394 (2006).

[69] P. J. Hesketh, C. Ju, S. Gowda, E. Zanoria, and S. Danyluk, "Surface free energy model of silicon anisotropic etching”, J. Electrochem. Soc. 140, 1080-1085 (1993).

[70] C. Thaulow, S. V. Schieffer, I. R. Vatne, D. Sen, and E. Ostby, "Crack tip opening displacement in atomistic modeling of fracture of silicon”, Computational Materials Science 50, 2621-2627 (2011).

[71] C. Chen and M. H. Leipold, "Fracture toughness of silicon", American Ceramic Society Bulletin 59, 469-472 (1980).

[72] Y. Wang and W. Yao, "Evaluation and comparison of several multiaxial fatigue criteria", Int. J. Fatigue 26, $17-25$ (2004).

[73] S. Hatanaka, K. Yasui, T. Kozuka, T. Tuziuti, and H. Mitome, "Influence of bubble clustering on multibubble sonoluminescence", Ultrasonics 40, 655-660 (2002).

[74] S. D. Gilev and A. M. Trubachev, "Metallization of silicon in a shock wave: the metallization threshold and ultrahigh defect densities", Journal of Physics: Condensed Matter 16, 8139 (2004).

[75] N. Bremond, M. Arora, S. M. Dammer, and D. Lohse, "Interaction of cavitation bubbles on a wall", Phys. Fluids 18, 121505 (2006).

[76] H. N. Oguz and A. Prosperetti, "Dynamics of bubble-growth and detachment from a needle.", J. Fluid Mech. 257, 111-145 (1993).

[77] R. P. H. M. Bergmann, D. van der Meer, S. Gekle, J. van der Bos, and D. Lohse, "Controlled impact of a disk on a water surface: cavity dynamics”, J. Fluid Mech. 633, 381-409 (2009).

[78] S. Gekle and J. Gordillo, "Compressible air flow through a collapsing liquid cavity", Int. J. Numer. Meth. Fluids 67 (2011).

[79] W. Bouwhuis, R. van der Veen, T. Tran, D. Keij, K. Winkels, I. Peters, R. van der Meer, C. Sun, J. Snoeijer, and D. Lohse, "Maximal air bubble entrainment at liquid drop impact", Phys. Rev. Lett. (2012).

[80] T. Leighton, The Acoustic Bubble (Academic Press, London) (1994).

[81] E.-A. Brujan, K. Nahen, P. Schmidt, and A. Vogel, "Dynamics of laser-induced cavitation bubbles near elastic boundaries: influence of the elastic modulus”, J. Fluid Mech. 433, 283-314 (2001). 


\section{A Simulation of a bubble collapse near a wall}

The collapse of a bubble can be simulated using an axisymmetric boundary integral (BI) method, which has been shown to be able to predict the collapse of single and multiple bubbles [75]. The numerical simulations assume potential (irrotational, incompressible, inviscid) flow around the bubble till the straight solid (impermeable) wall with length much larger than the initial bubble radius $R$. The axisymmetric bubble contour is described using cylindrical coordinates $r, z$ and is solved numerically The simulations are based on the numerical code described elsewhere [76-79]. The dynamic boundary condition on the droplet contours is the unsteady Bernoulli equation:

$$
\left(\frac{\partial \phi}{\partial t}+\frac{1}{2}|\nabla \phi|^{2}\right)=-g z-\frac{\gamma}{\rho_{l}} \kappa(r, t)-\frac{P_{e x t}-P_{\infty}}{\rho_{l}} .
$$

Here $\phi$ is the flow potential $(\nabla \phi=\vec{u}, \vec{u}$ being the local velocity vector), $t$ is time, $g$ the acceleration of gravity, $z$ the absolute height, $\gamma$ the surface tension, $\rho_{l}$ the density of the liquid, $\kappa(r, t)$ the interface curvature, $P_{e x t}(r, t)$ the external liquid pressure applied on the bubble, and $P_{\infty}$ the far field pressure. The initial conditions for the simulations consist of a spherical bubble with radius $R$, distance $d$ from the solid wall, under influence of a pressure $P_{\text {ext }}$. The BI code was checked by obtaining the evolution of the maximum bubble radius of a symmetric collapsing bubble (no wall, or $\mathrm{d}>>\mathrm{R}$ ) during the simulation. This radius-time curve was compared against the radius-time curve for a single, spherical bubble as predicted by the RayleighPlesset equation [80], simulated in MatLab using the ode45 function. The result is plotted in Figure 8.16 and shows good agreement between the two methods.

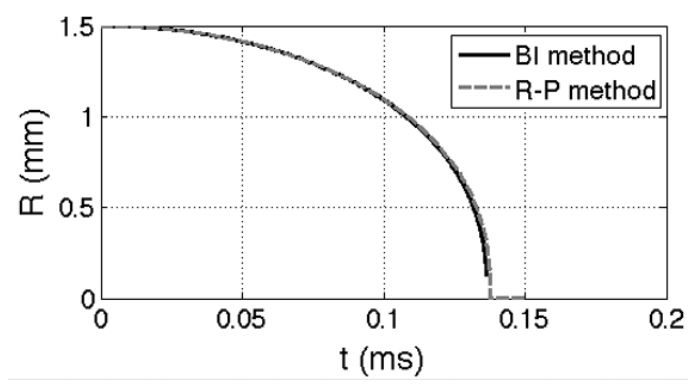

Figure 8.16: Radius-time curve for a $1.5 \mu \mathrm{m}$ initial radius bubble not wall-bound, as simulated with the BI code (black solid line) and a Rayleigh-Plesset model (gray dashed line).

Another validation was performed by simulating the collapse of a millimetersized laser-induced bubble near a solid wall, as studied by Brujan et al. [81], who reported a jet velocity of $130 \mathrm{~m} / \mathrm{s}$ for a bubble of radius $1.5 \mathrm{~mm}$ and a distance of 2.5 $\mathrm{mm}$ from the wall. The BI simulation resulted in a final velocity of order $\sim 10^{2} \mathrm{~m} / \mathrm{s}$. 
Closing Remarks and Outlook 


\subsection{Introduction}

The initial aim of the research program for this Thesis was not limited to the use of sonochemistry, but contemplated the use of other techniques. To obtain the main goal of this project -to improve the energy efficiency of sonochemical reactors by at least one order of magnitude-, as described in Chapter 1 , the way bubbles were to be formed could be by means of laser generation, monodisperse formation of microbubbles by flow-focusing devices, and hydrodynamic cavitation. In addition to what has already been presented in the preceding chapters, we will present some other preliminary results and description of experiments involving cavitation that are under preparation, or would certainly be interesting for future research.

\subsection{Interesting phenomena of bubbles and pits}

\subsubsection{Physical picture of bubble clusters interactions with other clusters and a wall}

As described in Chapters 3 and 6 , when more than one pit exists, and above a given pressure threshold, the bubbles in the cluster above each pit start to travel as streamers to the geometrical mid-point. Similarly, if the bubble cluster is in the vicinity of a boundary or wall, they travel towards it as in Chapter 7 . Qualitative explanations supported by preliminary experiments are given here with the aim to understand why the bubbles show this behavior. A numerical model that could be used to compare these preliminary experiments is still under preparation.

\section{One pit}

For simplicity, three different powers (low, medium and high) are chosen in similarity with the majority of the experiments presented in the rest of this Thesis. To our knowledge, we can define four main forces or interactions acting on the bubbles schematically represented in Figure 9.1 .

$F_{1 . \text { Bjerknes: }}$ The primary Bjerknes force, i.e. the radiation force from the acoustic wave generated by the piezo-container-liquid system. Is the average of the negative of the product between the pressure gradient and the bubble volume [1, 2]. When the bubbles are smaller than the resonant size, they are attracted to the antinode lying on the oscillating bottom of the container (to a great extent by the "image bubble" described later). Then as the power is increased, the bubbles grow larger and this situation may inverse.

$F_{\text {pit.bubble: }}$ The force between the ejected bubble and the bubble stabilized in the pit. Since the bubbles expelled are on average smaller (than resonant size) than the bubble in the pit (larger than resonant size), this force is repulsive when oscillating 

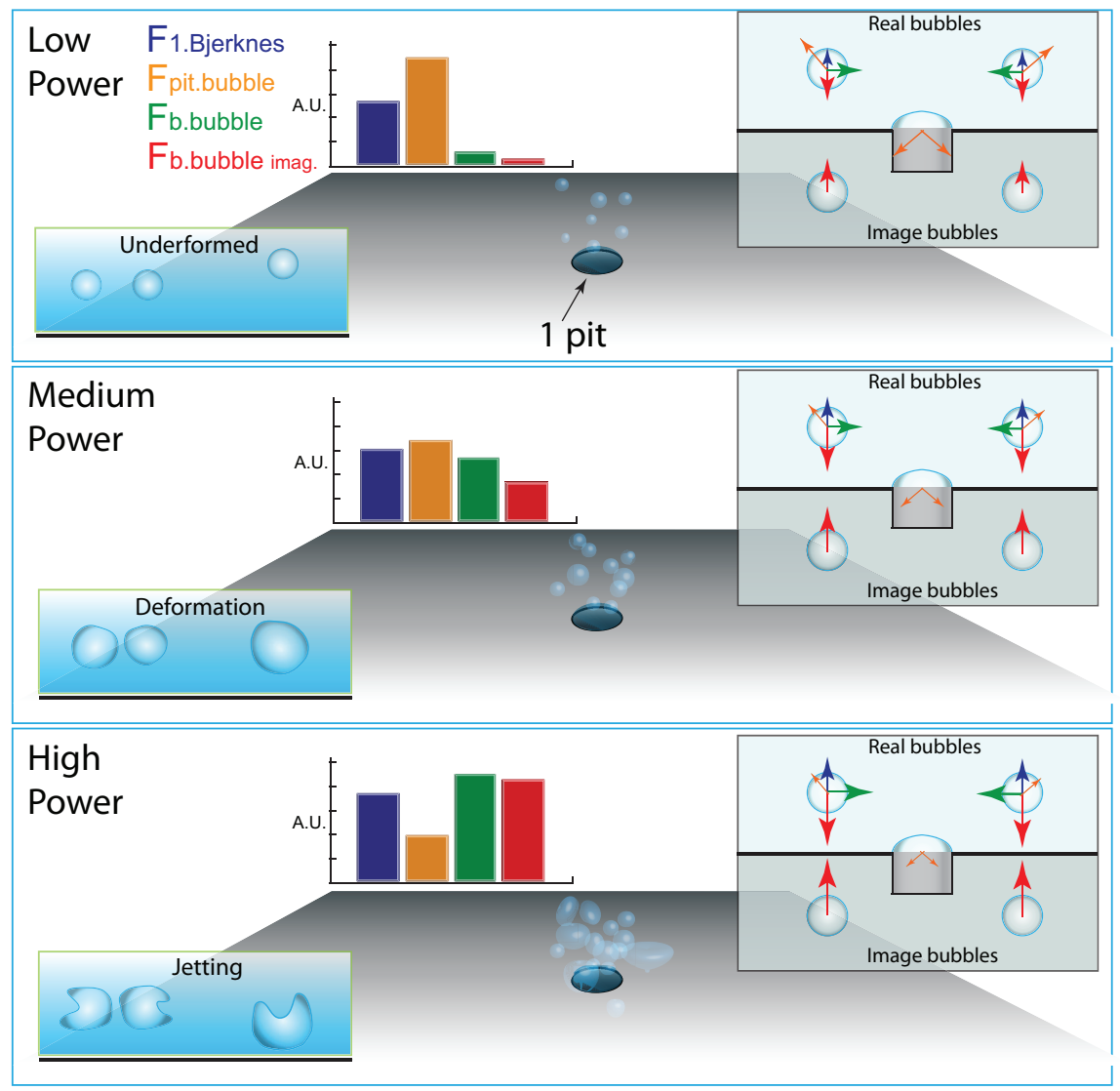

Figure 9.1: Simplified representation of forces involved and shapes of bubbles. The top chart accounts for low power settings; the name of the forces and colors associated to them are represented in arbitrary units in a bar plot. The inset to the top right depicts the force vectors described, while the bottom left inset shows the shape of the bubbles. The middle and bottom chart obtain similar information for different power settings.

in phase. As power increases and the expelled bubbles grow larger the repulsion decreases and eventually can start to attract. At higher power we observed bubbles breaking off in smaller bubbles, with a lifetime not larger than one cycle. For the 200 $\mathrm{kHz}$ frequency that we regularly used, bubbles smaller than $15 \mu \mathrm{m}$ in radius would be repelled by the pits, while larger bubbles would be attracted (see Chapter 6).

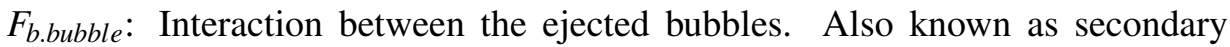
Bjerknes force. The ejected bubbles are approximately the same size and are attracted to each other if oscillating in phase.

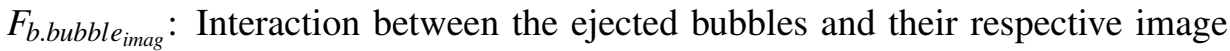
bubble. This stands for the attractive forces towards the nearby boundary experienced 
by the bubbles as power is increased, and would be similar in explanation as $\mathrm{F}_{b . b u b b l e}$. This is sometimes recalled as a "Narcissus effect" [3].

With increasing power, the relative contribution of forces changes as represented in the Bar plots and vectors in Figure 9.1.

\section{Two pits}

When varying the voltage (pressure amplitude) of the ultrasound signal, there seems to be some "hysteresis" in the way the clusters connect or disconnect forming the interesting streamer paths (described in Chapter 6). The "attachment" voltage $\mathrm{U}_{a}$ is larger than the "detachment" voltage $\mathrm{U}_{d}$, and this is what we call "hysteresis". In this case we need to add another interaction: cluster-cluster and cluster-image cluster. Figure 9.2 attempts to explain the interactions for the case of two pits in a simplified way, and also a proposed artistic representation of the dependence of the presumed interaction forces.

A model able to describe this behavior is currently being studied. The clustercluster interactions were also described in Chapters 4 and 6.

\subsubsection{Presence of a wall}

When a wall is near a pit with a bubble cluster cavitating, the situation is similar as when there are two pits, since the "real cluster" feels the presence of the "image cluster". While keeping the wall at a given distance, increasing the power, the bubble streamers travel to the wall at a voltage higher than at which they detach, another type of hysteresis. Figure 9.3 depicts the interactions for the case of one pit near a wall in a simplified way.

\section{Presence of a moving wall}

Similarly, while keeping the voltage constant, moving the wall closer to the cluster of bubbles around the pit, the bubbles "attach" to the wall at a distance smaller than at which they "detach". A simplified sketch is depicted in Figure 9.4. The fact that the "detachment" distance $\mathrm{L}_{d}$ is larger than the "attachment" distance $\mathrm{L}_{a}$ can also be called "hysteresis".

\subsubsection{Variation of the distance between the pits}

The phenomena described for the variation of pressure (voltage) for two pits, or one pit with a wall close to the pit, is somewhat similar to when varying the distance between the pits while keeping the voltage constant. For a larger separation between the pits, the voltage needed to "connect" the streamers is higher. In this case nevertheless, the situation cannot be compared directly since, since the pits are separated 


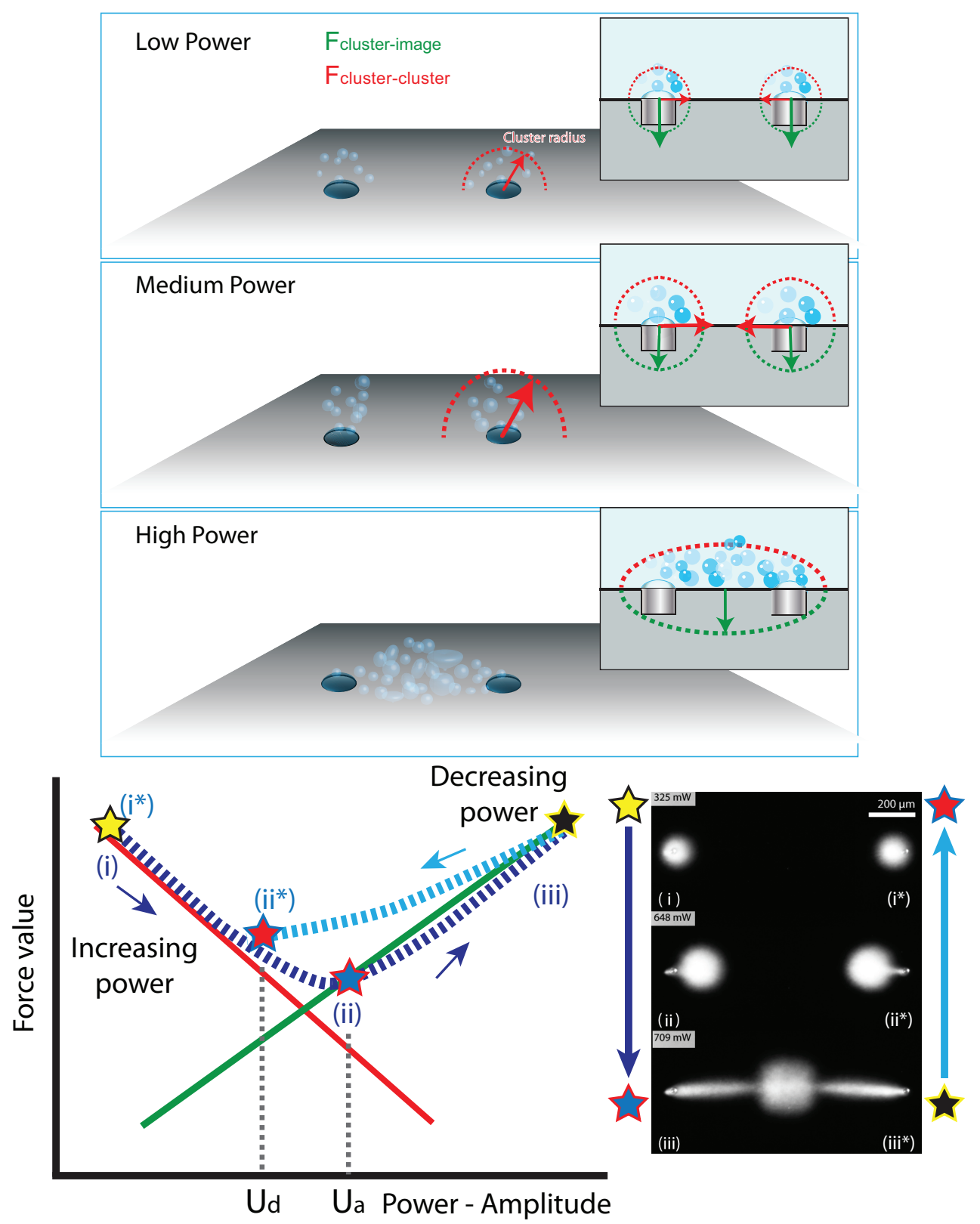

Figure 9.2: Schematics for the "interaction forces" of two clusters nucleated from pits as the power is varied. In red the curve of the attraction forces within one cluster and in green the attractive forces between the two clusters. As the power is increased the interaction between each real cluster increases and as the streamers travel to the geometrical mid-point, the interaction with the image bubbles also increases. Disclaimer: The shape of the curves might not be correct, they are plotted for illustrative purposes. 


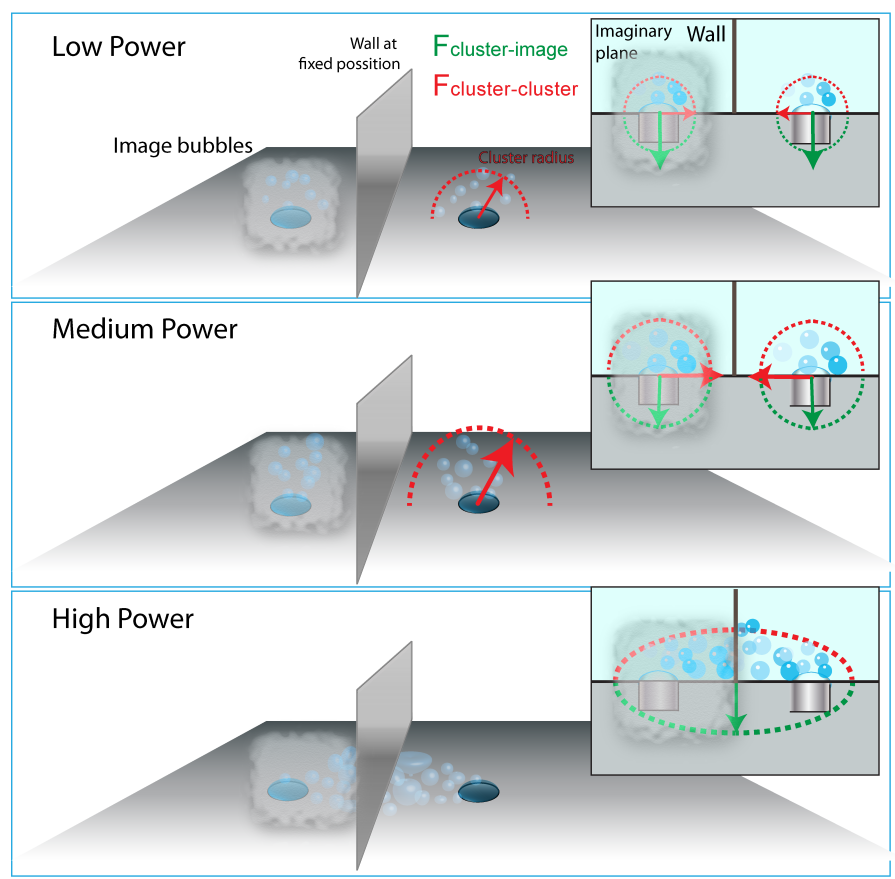

Figure 9.3: Simplified representation of forces involved and bubble clusters with a wall fixed as the power is increased.

farther apart, different pressure gradient exists and the bubbles will not behave the same as in the center of the cavitation cell.

\subsubsection{Pits with different shapes}

One idea to control the conditions at which a bubble can cavitate, is to use a microscopical equivalent of the one dimensional bubble defined by Leighton and collaborators [4]. In their experiment they show how a conical shape end of a tube can stabilize a bubble at its apex. When a meniscus oscillates in a piston-like fashion, at the apex of the cone, SL, SCL and several sonochemical effects occur. In Fig. 9.5 a schematics of a conical-planar reactor concept is shown; the operation principle could be either US irradiation in a similar fashion as with the pits by gluing a piezo to the bottom, or perhaps by making the liquid flow oscillate. The top side of the etched silicon substrate would be capped by a glass slide.

Before such conical-planar reactor was created, a first attempt was done with wet-etching of pyramidal pits in silicon (100) substrates. Interestingly, instead of achieving a piston like movement, ejection of bubbles like in the case of cylindrical 

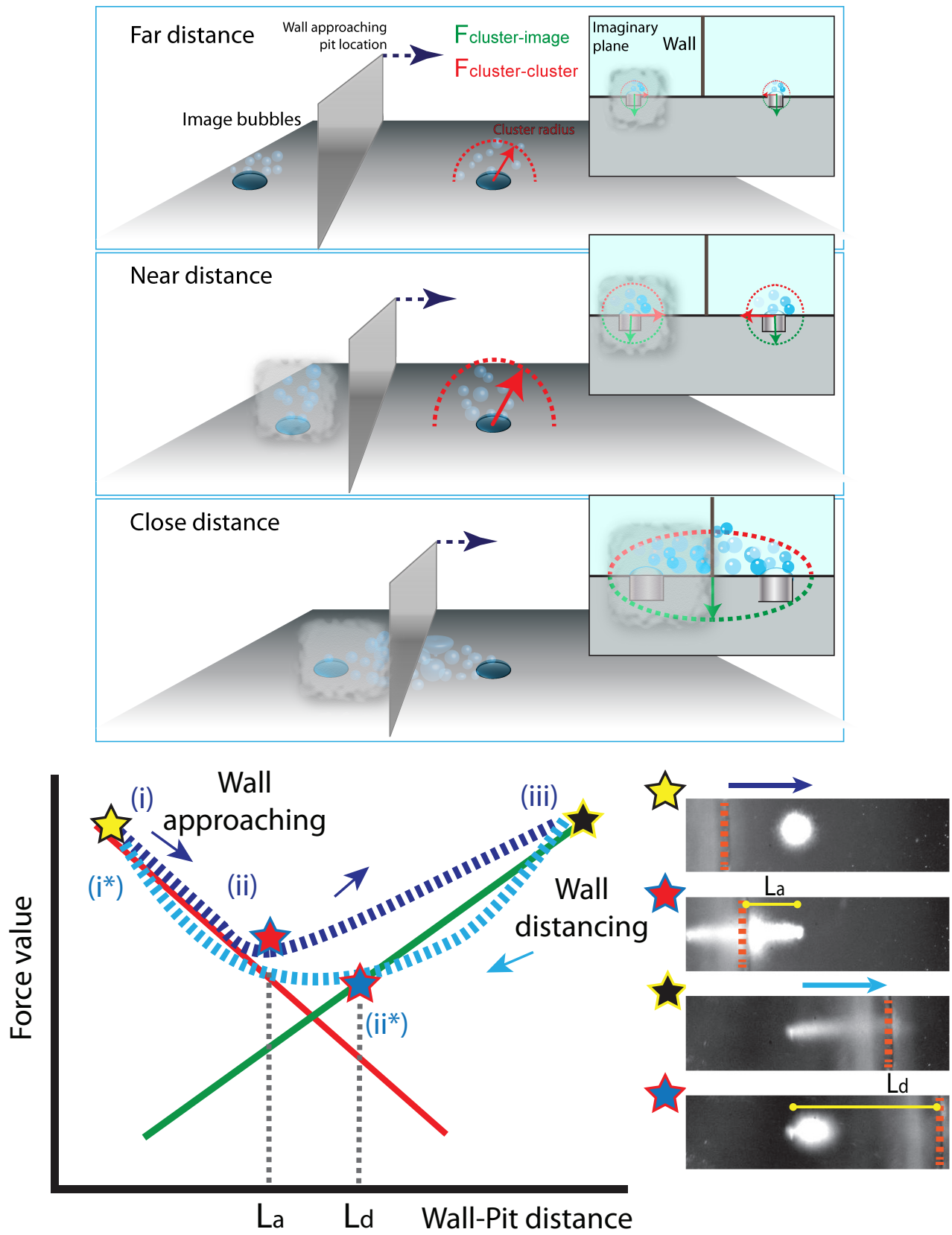

Figure 9.4: Schematics for the "interaction forces" for a cluster nucleated from one pit as the power is constant and a wall moves over it. In red the curve of the attraction forces within the real cluster and in green the attractive forces between the real and image clusters. Disclaimer: The shape of the curves might not be correct, they are plotted for illustrative purposes. 


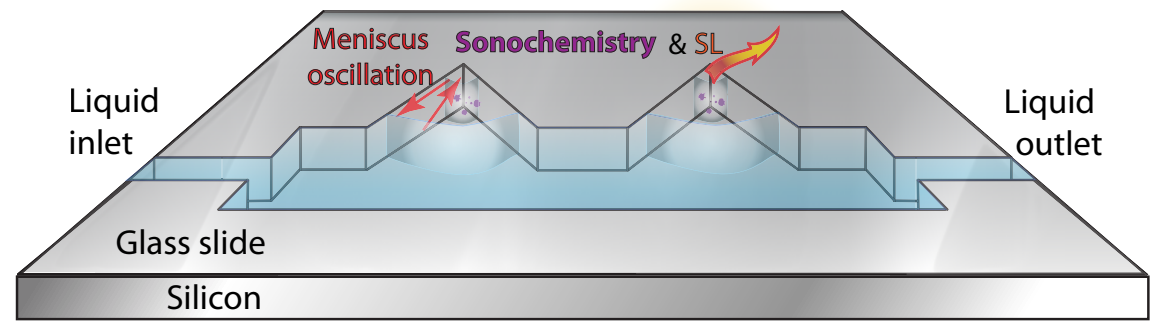

Figure 9.5: Prototype microdevice for the conical-planar bubble experiment.

pits took place, but apparently from the corners of the squared rim of the pyramidal pit. It was not possible to micromachine conical micropits, but it is expected that a conical geometry would increase the compression ratio of the conical bubble compared to a cylindrical bubble.

Additionally, inspired by the observations of bubble ejection from pyramidal shaped pits described in the previous paragraph, we recorded the initial oscillations of gas bubbles stabilized in artificial pits on a substrate having different geometrical configurations. The ultrasonic frequency was $200 \mathrm{kHz}$, the ultra-fast imaging camera Brandaris was operated at ca. $10 \mathrm{Mfps}$, and triggered to record the initial oscillations of the bubble in the pit. During the initial pressure oscillation cycles, the behavior of the liquid-gas meniscus for all pit shapes was similar to the drum-like vibration. After a certain amount of cycles and presumably amplitude increase, the bubble expanded gradually, until certain characteristic bubble shapes started to appear depending on the shape of the pits. The continued expansion and deformation of the pit resulted eventually in microbubble pinch-off. Once the first bubbles are ejected, the scenario does not differentiate significantly from the cylindrical pit case. See Fig. 9.6 for a square pit and a circular pit with a rim around.

\section{Roughness of the inner surface of a pit}

When the inner walls of the pit are roughened (scalloped as a result of the etching process used: Bosch Deep Reactive Ion Etching, DRIE) the bubble is stabilized in the pit as for the results shown on this Thesis. When pits are etched with a machine that leaves the inner wall of the pits smooth (cryogenic DRIE), the bubble trapped in the pit behaves differently. It seems that the meniscus contact line is allowed to move along the inner surface with less resistance. Some experiments were started after $\sim 5$ min from the moment of pouring the water. In this case, the bubble grew (presumably by rectified diffusion) and detached from the pit rising up to the free 


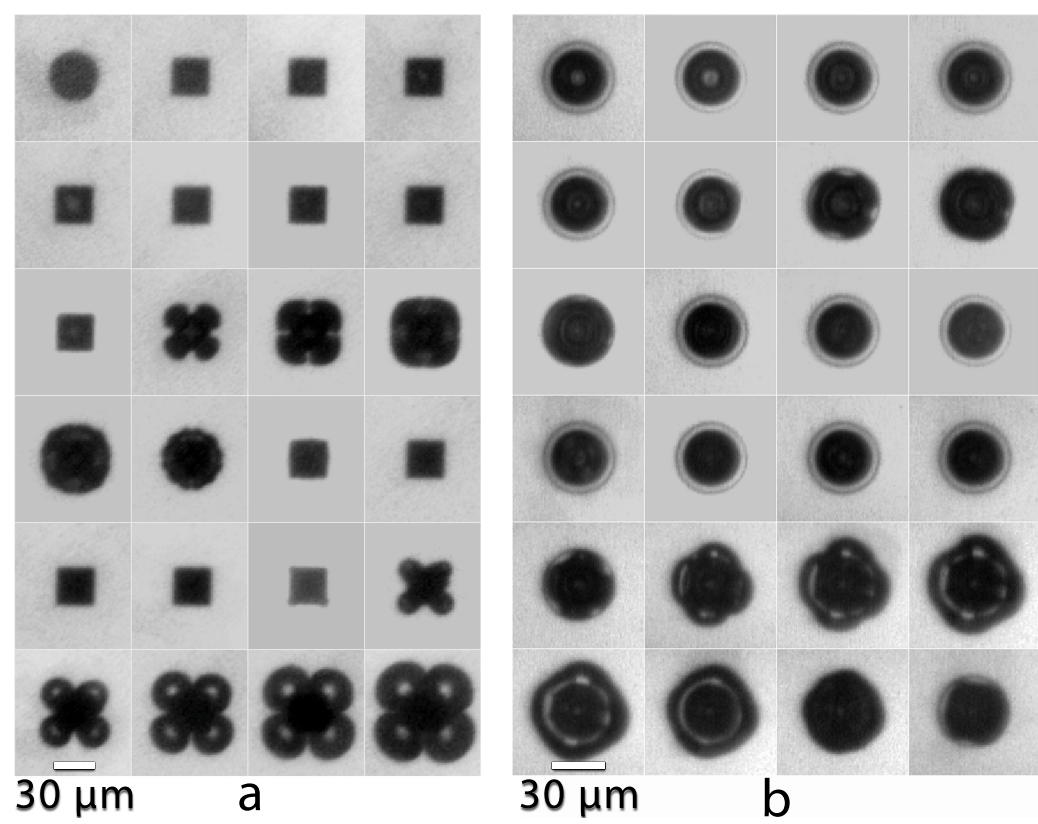

Figure 9.6: First oscillations of a bubble trapped in a) a square pit; b) a circular pit with a rim etched around. Every four frames approximately a new acoustic cycle takes place as it was recorded at 4 Million frames per second [5].

surface sometimes before the ultrasound would be started. In several experiments the bubble detached from the walls of the pit and remained cavitating at the bottom.

\subsection{Novel sonochemistry}

\subsubsection{Monodisperse bubbles}

Ultrasound is used in medicine for imaging and drug delivery with the help of ultrasound contrast agents (UCA). UCA, are microbubbles and their generation and size can be controlled; this feature could lead to interesting sonochemical applications avoiding cluster-cluster interactions. As shown before in this Thesis, these interactions affect the efficiency of micro-sono-reactors. A microfluidic device able to generate microbubbles with radii ranging from 12 to $15 \mu \mathrm{m}$ has been built to sort microbubbles on-line in a traveling ultrasound wave [6]. This sorting device can be coupled to another piezo transducer or group of transducers and in this way, the cavitation of the generated bubbles can lead to sonochemical products in a more controllable way as presented in Figure 9.7 . 


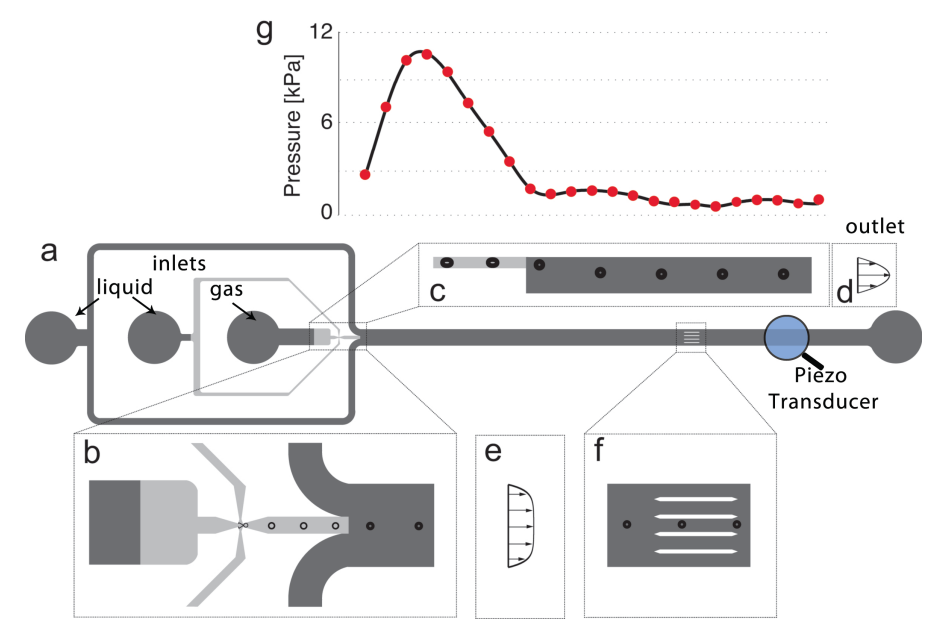

Figure 9.7: (a) Schematic drawing of the fluidic device. (b) Microbubbles are formed in a flow focusing geometry (c) that emanates in a deeper main channel allowing the bubble to attain a spherical shape. (d) shows the parabolic flow profile in the height direction and (e) the mainly constant velocity profile over the width of the channel. $(f)$ shows the outlet channel over which the bubbles can be sorted. Piezo transducers can be located on different position along the channel, each having a different frequency depending of which bubble size is being generated. Another type of transducers could be used to make the sorted bubbles cavitate and produce chemical reactions instead for sorting the bubbles. Adapted from [6]

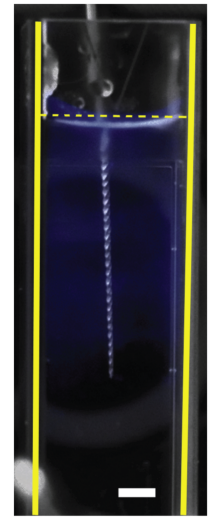

(a)

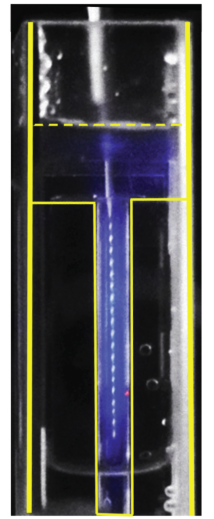

(b)

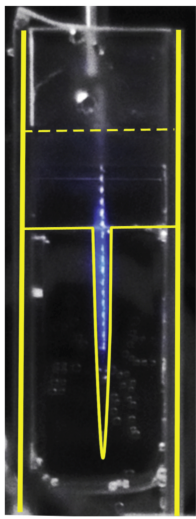

(c)

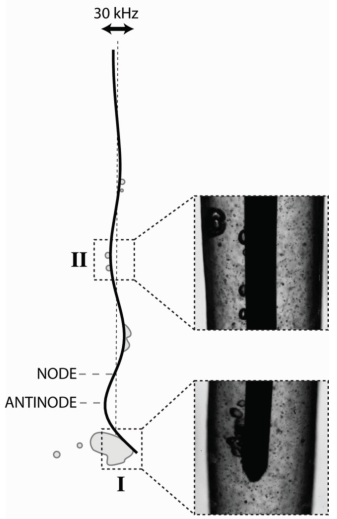

(d)

Figure 9.8: Overlay of luminol photos (blue) with ambient light photos, showing the spatial distribution of luminol inside the cuvette (a) and bovine-sized (b) and human-sized (c) root canal models. The confinements have been outlined with solid yellow lines; the luminol-air interface is indicated with a dashed yellow line. The white bar in (a) is $2.5 \mathrm{~mm}$ wide. d) Sketch of the occurrence of cavitation along the length of an oscillating file. At the tip of the file (I), a large cavitation bubble cloud is formed; at other antinodes (II), only small, single bubbles are observed. The file is oscillating inside the confinement of a root canal model 


\subsubsection{Towards new dental cleaning tools}

Cavitation has been associated with certain effects in dental treatments, but the nature and characteristics of the cavitating bubbles was not yet fully elucidated. Using sensitive equipment, the SL and SCL around these files have been measured, showing cavitation occurring even at a very low power setting. Luminol photography and high-speed visualizations provided information on the spatial distribution of the cavitation bubbles (see Fig. 9.8).

A large bubble cloud was observed at the tip of the files, but this was found not to contribute to SCL. Rather, smaller, individual bubbles observed at antinodes of the oscillating file with a smaller amplitude were leading to SCL. Confinements of the size of bovine and human root canals increased the amount of SL and SCL compared to a very large confinement. However, the root canal models also showed the occurrence of air entrainment, resulting in the generation of droplets and stable bubbles near the air-liquid interface and eventual loss of liquid [7].

\subsubsection{Potential new sonochemical reactors}

\section{Flow-through micro-sono-reactor}

Sonochemical practical applications normally require higher conversion rates than those attained with the micro-sono-reactor shown in this Thesis. A solution might be a microchamber or microchannel with pits etched on any of its surfaces; but in this case with a liquid inlet and outlet. A recirculation loop and a given number of passes may be used to reach the desirable conversion value as suggested in Chapter 2

\section{Sparger with gas control}

In combination with the previous flow-through micro-sono-reactor concept, there are several sonochemical applications where the control over the type and amount of dissolved gas is crucial. Several applications like synthesis of nanoparticles and advanced oxidation processes can benefit from a reactor in which different gases could be used in a sparging regime. In Fig. 9.9 we present the reactor built for this aim.

Currently we are studying a way to increase the energetic potential of low-grade biofuels like guaiacol, one compound found in lignin. Hydrodeoxygenation is one of the most promising routes to upgrade guaiacol. It involves the hydrogenation of guaiacol, in which the oxygenated bonds react with the hydrogen to form water. Normally high temperatures and pressures are required. With this micro-sonoreactor-sparger, and at room temperature, this reaction can be obtained with several advantages like low reactants consumption and better safety conditions of operation conditions. Since hydrogen radicals are formed as a result of bubble cavitation, we may achieve the hydrodeoxygenation of guaiacol without the need for a catalyst. 

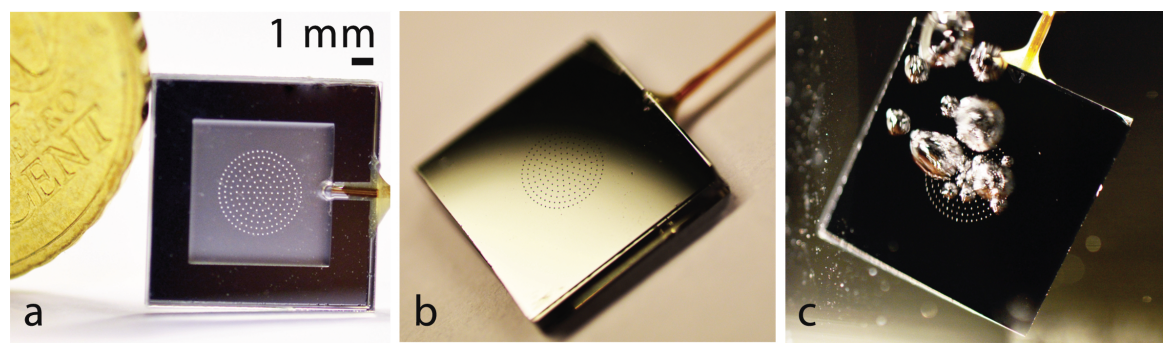

Figure 9.9: a) Photograph of the sono-micro-reactor with gas chamber on the back. $b$ ) Front side. c) Gas bubbles are formed when the reactor is immersed in water and gas is flown through the pits of $30 \mu$ m diameter.

a

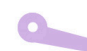

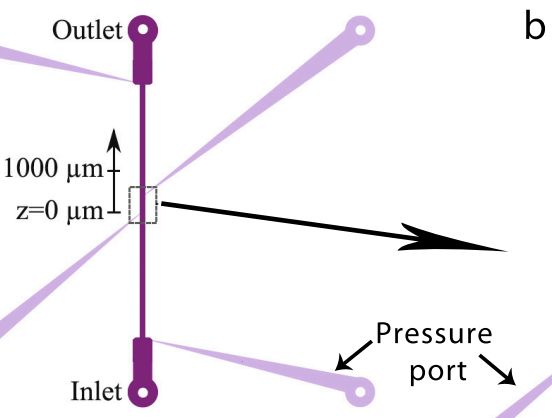

C

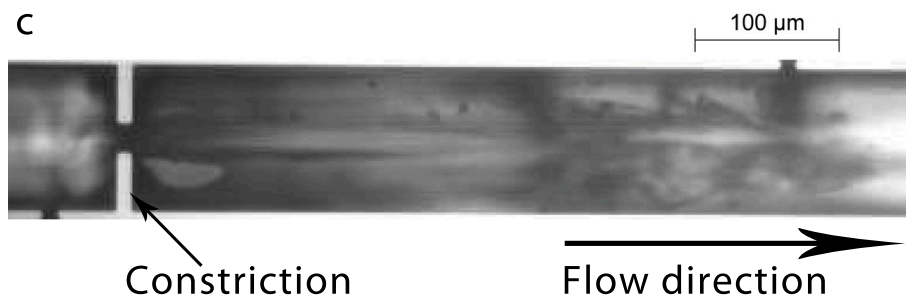

d

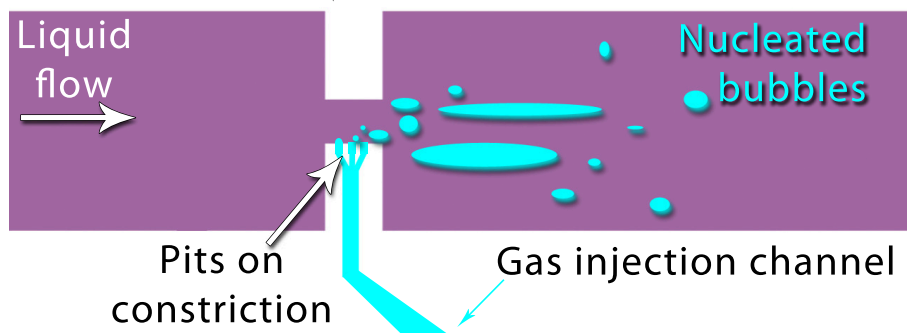
constriction b

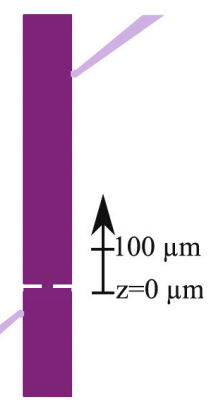

$100 \mu \mathrm{m}$

Flow direction

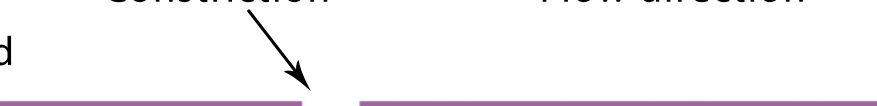

Figure 9.10: $a$, Schematic top view representation of the channel layout; $b$, entire channel; c, magnification of the constriction. The main channel with the constriction is in the center (dark color). The diagonal channels are connected to pressure sensors or dead ends (light colour)(taken from [8]). d, Proposed new concept for hydrodynamic cavitation with micromachined pits. 


\subsubsection{Hydrodynamic cavitation and sonochemistry in a microreactor}

In previous reports we gave results on the study of hydrodynamic cavitation in a millichannel and microchannel with constrictions [8]. There was no measurable chemical conversion for the case of the microchannel. To resolve this, we propose for future research the combination of a similar microchannel with pits. In this case we expect that the flow rates and pressures needed to obtain hydrodynamic cavitation could be lower. Additionally, the combination of such a device with a glued piezo could be feasible, that when operated at a certain frequency, could improve the generation of hydrodynamic cavitation. A schematics is given in Figure 9.10 .

\subsubsection{SAW and pits}

Surface Acoustic Waves (SAW) have gained attention in the manipulation of particles and other microfluidic applications [9]. In a similar way as the pinch-off using US sonication with a piezo glued to a microchamber, a SAW device could be used to promote the pinch-off of bubbles [10].

\subsection{Flow and transport characterization induced by pit-stabilized bubbles}

\subsubsection{Transport of Brownian particles driven by bubble microstreaming flow}

In semiconductor fabrications, a commonly adopted wafer cleaning process is megasonic cleaning. As has been described several times in this Thesis, bubbles can damage the surface of silicon wafers if the power is high enough (see Chapter 8). To achieve a damage-free cleaning performance with low acoustic powers, a fundamental understanding of the cleaning mechanisms is necessary. The microstreaming flow and the capillary force induced by bubbles that are randomly sweeping on the substrate are the main dominant mechanisms for the removal of submicron particles from a wafer substrate. In Figure 9.11 a processed image and transport speeds are presented. The feasibility of controlling a microstreaming flow to promote particle transport in the close vicinity of a stationary substrate can be achieved with micomachined pits. As the gas pocket (bubble in the pit) oscillates, the fluid above it will be set into motion. The experimental tracking of colloidal gold particles and its comparison with numerical simulations is the aim of an ongoing collaboration [11]. The most effective region for particle transport (strongest flow) is found around the pit bubble and decays with distance. The actual range of effective particle transport can vary for different particle size, acoustic power and bubble dimension, etc. As the 
bubble microstreaming flow decays with distance, Brownian motion dominates for particles far away from the bubble.

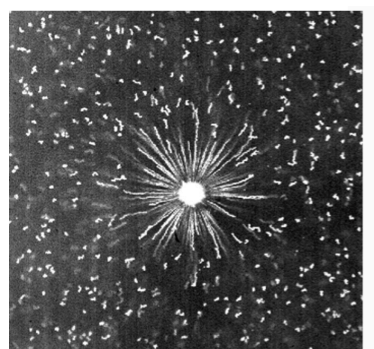

a)

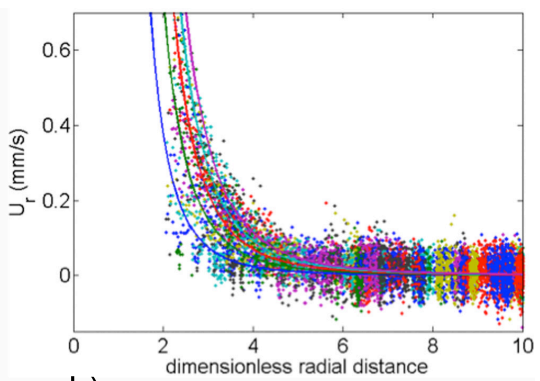

b)

Figure 9.11: Particle transport within $5 \mu \mathrm{m}$ distance to the substrate induced by bubble microstreaming flow driven at $256.1 \mathrm{kHz}$ and $20 \mathrm{kPa}$. a) Top view streaklines from a superposition of 250 successive frames, the recording frame rate is 250 fps. b) Radial transport speeds obtained from particle tracking velocimetry plotted against the radial distance to bubble center normalized by the radius of the micropit. The solid lines are the fitted theoretical radial speeds at 1 to $5 \mu \mathrm{m}$ distance away from the substrate, the fitted microstreaming velocity scale is $15 \mathrm{~mm} / \mathrm{s}$

Particles that are loosely attached to the substrate are carried by the strong vortex, move towards the oscillating bubble, and get removed from the substrate upon approaching the bubble. When driven by low acoustic power, the induced flow intensity shows a quadratic dependence on the driving pressure due to the secondary streaming effect. Brownian dynamics simulation shows an excellent agreement with the experiments. At high acoustic power, the bubble meniscus becomes unstable but the flow intensity is greatly enhanced by some nonlinear effect that turn into bubble pinch-off.

\subsubsection{Astigmatism Particle Tracking in micropits}

Another ongoing project is the accurate measurement of the flow generated by acoustically streaming bubbles and the bubble trapped in the pits. For this main objective we plan to use the 3D A-PTV (Astigmatism Particle Tracking Velocimetry) technique developed at the Microfludics Lab from the Bundeswehr Universität in Munich [12]. This technique follows a simple strategy to resolve 3D flows using a Particle Tracking approach with one single camera, which can be adapted in any experimental set-up.

\subsection{Closing remarks}

With a relatively simple modification of the surface of a micro-sono-reactor, an order of magnitude increase in operation efficiency under laboratory conditions was 
achieved; this the most important result of this Thesis. With such a "passive modification" of a surface several scenarios are possible: increasing the number of pits, the area over which the pits are located, the power, type of gas, positioning inside a given reactor, continuous or batch operation, temperature control, operation frequency, and others. Depending on which application is required, new reactors can be designed and tested keeping in mind the interrelated factors described in this Thesis. Hopefully new researchers and engineers will pick up some of the ideas and experience provided here. Then the development of "greener solutions" with the use of microfluidics and sonochemistry will benefit our society.

\section{References}

[1] K. Yasui, Y. Iida, T. Tuziuti, T. Kozuka, and A. Towata, "Strongly interacting bubbles under an ultrasonic horn", Phys. Rev. E 77, 016609 (2008).

[2] T. J. Matula, S. M. Cordry, R. A. Roy, and L. A. Crum, "Bjerknes force and bubble levitation under singlebubble sonoluminescence conditions”, J. Acoust. Soc. Am. 102, 1522-1527 (1997).

[3] P. Marmottant, M. Versluis, N. de Jong, S. Hilgenfeldt, and D. Lohse, "High-speed imaging of an ultrasounddriven bubble in contact with a wall: "Narcissus" effect and resolved acoustic streaming", Exp. Fluids 41, 147-153 (2006).

[4] T. G. Leighton, B. T. Cox, and A. D. Phelps, “The rayleigh-like collapse of a conical bubble”, J. Acoust. Soc. Am. 107, 130-142 (2000).

[5] D. Fernandez Rivas, G. Lajoinie, B. Verhaagen, J. G.E. Gardeniers, M. Versluis, and D. Lohse, "Strange pits: first oscillation of bubbles stabilized in pits", unpublished (2012).

[6] T. Segers, W. van Hoeve, D. Fernandez Rivas, and M. Versluis, "Acoustic bubble sorting: manipulation of microbubbles by primary radiation forces", to be submitted to Lab-on-Chip (2012).

[7] R. Macedo, B. Verhaagen, D. Fernandez Rivas, H. J. Gardeniers, L. van der Sluis, P. Wesselink, and M. Versluis, "Sonochemical and visual characterization of cavitation generated by ultrasound in root canal models", Ultson. Sonochem to be submitted (2012).

[8] J. Rooze, M. André, G.-J. van der Gulik, D. Fernandez Rivas, J. Gardeniers, E. Rebrov, J. Schouten, and J. Keurentjes, "Hydrodynamic cavitation in micro channels with channel sizes of 100 and 750 micrometers", Microfluid Nanofluid 12, 499-508 (2012).

[9] J. Friend and L. Y. Yeo, "Microscale acoustofluidics: Microfluidics driven via acoustics and ultrasonics", Rev. Mod. Phys. 83, 647-704 (2011).

[10] J. Friend, Personal Communication (2011).

[11] X. Xu, D. Fernandez Rivas, A. G. Zijlstra, S. Brems, P. Mertens, H. Struyf, J. G. E. Gardeniers, and D. Lohse2, "Transport of brownian particles driven by bubble microstreaming flow", (2012), to be submitted to APL.

[12] C. Cierpka, M. Rossi, R. Segura, and C. J. Köhler, "On the calibration of astigmatism particle tracking velocimetry for microflows", Meas. Sci. Technol. 22, 015401 (2011). 


\section{Summary}

This Thesis shows that through microfabrication techniques, an improved efficiency of sono-reactors can be achieved by the control of the nucleation sites of bubble streamers. It has been proofed that one order of magnitude improvement compared to the equivalent conventional sonoreactor is feasible.

The characteristics of the light emitted (SL and SCL) from the reactor can give useful information on the type of bubbles generated. When more sophisticated techniques are available, more details on the nucleation of bubble streamers and its complex behavior can be addressed.

The practical uses of these bubble streamers is shown for removing various types of layers and in different liquids other than water. The negative effects of cavitation erosion were studied for different types of silicon with important insight into future reactors design strategies.

Nevertheless, the complexity of sonochemical reactors still remains, as bubbles generated in this way do not behave in a simpler way than in conventional reactors. What is clear is that there is enough room for improvement and challenging and new applications are around the corner to further tame acoustic cavitation. 


\section{Samenvatting}

Dit proefschrift laat zien dat sonoreactoren efficiënter kunnen worden gemaakt door de positie voor nucleatie van cavitatiebellen te controleren met behulp van microfabricage. Bewezen werd dat een verbetering met een factor 10 ten opzichte van gelijkwaardige conventionele sonoreactoren mogelijk is.

Karakterisering van het licht dat ten gevolge van sonoluminescentie en sonochemiluminescentie vanuit een reactor wordt uitgezonden kan nuttige informatie geven over de bellen die gegenereerd worden. Daarnaast is met behulp van hogesnelheids opnamen en met simulaties het bijzondere gedrag van de bellen en de belnucleaties onderzocht.

Enkele praktische toepassingen van de inklappende bellen zijn gedemonstreerd, in water en in andere vloeistoffen, zoals het verwijderen van verschillende soorten lagen die op een oppervlak waren aangebracht. De negatieve effecten van cavitatie (erosie) werden ook bestudeerd voor verschillende siliciumsubstraat- oriëntaties, wat belangrijke inzichten opleverde voor het ontwerp van toekomstige reactoren.

Ondanks al deze nieuwe informatie blijft de complexiteit van sonochemische reactoren bestaan, aangezien de op genoemde wijze gegenereerde bellen zich op dezelfde complexe wijze gedragen als in conventionele reactoren. Wat echter duidelijk is geworden is dat er mogelijkheden zijn voor meer controle over en verbetering van sonoreactoren, en dat er interessante nieuwe toepassingen binnen bereik komen door het temmen van akoestische cavitatie. 
List of Publications during PhD 


\section{List of Publications during PhD}

\section{Published Papers}

- Fernandez Rivas, D., Cintas, P., Gardeniers, J.G.E., Merging microfluidics and sonochemistry: towards greener and more efficient micro-sono-reactors. In typesetting in Chem. Commun., DOI: 10.1039/C2CC33920J (2012).

- Fernandez Rivas, D., Verhaagen, B., Seddon, J., Jiang, L.M., Zijlstra, A. G., van der Sluis, L., Versluis, M., Prosperetti, A., Lohse, D. and Gardeniers, J.G.E. Localized removal of deposited layers by cavitation microbubbles. Biomicrofluidics, (6), 034114 (2012).

- Fernandez Rivas, D., Stricker, L., Zijlstra, A. G., Gardeniers, J.G.E., Lohse, D. and Prosperetti, A. Ultrasound nucleated bubbles and their sonochemical radical production. Accepted for publication in Ultrasonic Sonochemistry in press, doi:10.1016/j.ultsonch.2012.07.024, (2012).

- Fernandez Rivas, D., Ashokkumar, M., Leong, T., Yasui, K., Tuziuti, T., Kentish, S., Lohse, D. and Gardeniers, H.J.G.E. Sonoluminescence and sonochemiluminescence from a microreactor Ultrasonic Sonochemistry. (19) 1252-1259. (2012).

- Rooze, J., Matthieu André, Gert-Jan S. van der Gulik, David Fernández Rivas, Johannes G. E. Gardeniers,Evgeny V. Rebrov, Jaap C. Schouten and Jos T. F. Keurentjes Hydrodynamic cavitation in micro channels with channel sizes of 100 and 750 micrometers. Microfluidics and Nanofluidics, Springer. Issn: 1613-4982. DOI: 10.1007/s10404-011-0891-5, (2011).

- Fernandez Rivas, D., A. Prosperetti, A.G. Zijlstra, D. Lohse, J. G. E. Gardeniers Efficient Sonochemistry through Microbubbles Generated with Micromachined Surfaces, Angewandte Chemie International Edition, 49: 9699 - 9701. doi: 10.1002/anie.201005533, (2010).

- Fernández Rivas, D., Microfluidos: Nuevas fronteras, Revista Cubana de Física, Vol. 28, No. 1. (2011).

- Fernández Rivas, D., Microfluidos: ¿Cuánto hay de nuevo?, Revista Cubana de Física, Vol. 25, No. 2B. p 142-149. (2008).

- Kashid, M., D. Fernández Rivas, D. W. Agar and S. Turek, On the hydrodynamics of liquid-liquid slug flow capillary microreactors, Asia-Pacific Journal of Chemical Engineering, 3: 151-160. doi: 10.1002/apj.127. (2008). 
- Fernández, D. and Gardeniers, J.G.E., On the resilience of PDMS microchannels after violent optical breakdown microbubble cavitation, 6th International Conference on Nanochannels, Microchannels, and Minichannels, ICNMM2008; Darmstadt Germany. (2008).

\section{Papers Submitted or in Preparation}

- Fernandez Rivas, D., Betjes, J. Verhaagen, B., Lohse, D. and Gardeniers, J.G.E., Erosion evolution in mono-crystalline silicon surfaces caused by acoustic cavitation bubbles To be submitted to J. Appl. Physics. (2012)

- Zijlstra, A.G., Fernández Rivas, D., Gardeniers, J.G.E., Versluis, M. and Lohse, D., Enhancing acoustic cavitation using artificial crevice bubbles, To be submitted, (2012).

- Xu, X., Fernandez Rivas, D., Zijlstra, A. G., Brems, S., Mertens, P., Struyf, H., Gardeniers, J.G.E. and Lohse, D., "Transport of brownian particles driven by bubble microstreaming flow", to be submitted to APL, (2012).

- Macedo, R.G., Verhaagen, B., Fernandez Rivas, D., Gardeniers, J.G.E., van der Sluis, L., Wesselink, P. and Versluis, M., Sonochemical and visual characterization of cavitation generated by ultrasound in root canal models, to be submitted to Ultson. Sonochem. (2012).

- Macedo, R.G., Nikitenko, S.I., Verhaagen, B. Fernandez Rivas, D., Gardeniers, J.G.E., Versluis, M., van der Sluis, L., "Sonochemical dosimetry with tertButyl Nitrite and its potential application in dental research", to be submitted to Ultrason. Sonochem., (2012).

\section{Poster and Presentations (since 2007)}

- Microneedles 2012, Cork, Ireland, 13-15 May, 2012. Poster.

- Micromolded nanoporous ceramic microneedle arrays. Co-authors: J. de Groot, M. Verhoeven,T.D. de Gruijl, R.J. Scheper and R. Luttge.

- Acoustic Waves for the Control of Microfluidics Flows, Lorentz Center, Leiden, The Netherlands. April 23-27, 2012.

- Ultrasound nucleated bubbles in a microreactor: radical production, sonoluminescence, sonochemiluminescence and cleaning control. Various authors. 
- $20^{\text {th }}$ Annual Meeting of the Japan Society of Sonochemistry (JSS) and The International Workshop on Advanced Sonochemistry, Nagoya, Japan, November 2-4, 2011.

- Sonoluminescence and sonochemiluminescence in microreactors. Coauthors: T. Leong, M. Ashokkumar, D. Lohse, J.G.E. Gardeniers.

- XV Convención Científica de Ingeniería y Arquitectura CUJAE La Habana, Cuba. November $29^{\text {th }}$ December 2010. Two Oral presentations.

- Chemical processing and analysis using microfluidic systems. Co-author: J.G.E. Gardeniers.

- Microfluidic Hydrodynamic Cavitation. Co-authors: J. Rooze, M. Andre and J.G.E. Gardeniers.

- The 14th International Conference on Miniaturized Systems for Chemistry and Life Sciences 3 - 7 October 2010. Oral presentation.

- Sonochemical microreactor with microbubbles created on micromachined surfaces. Co-authors: A.G. Zijlstra, D. Lohse, A. Prosperetti and J.G.E. Gardeniers

- $20^{\text {th }}$ International Congress on Acoustics, ICA 2010, Sydney, Australia.23-27 August 2010. Oral presentation.

- Sonochemistry with micromachined pits. Co-authors: A.G. Zijlstra, D. Lohse, A. Prosperetti and J.G.E. Gardeniers

- $2^{d o}$ Seminario internacional de Nanociencias y Nanotecnologías. La Habana, Cuba. 14 al 17 de noviembre de 2008.

- Sixth International ASME Conference on Nanochannels, Microchannels and Minichannels Darmstadt, Germany. 23-25 June 2008. Poster.

- On the resilience of PDMS microchannels after violent optical breakdown microbubble cavitation. Co-author: J.G.E. Gardeniers.

- Physics of Micro- and Nanofluids. Leiden, The Netherlands. From 9 - 20 Jun 2008.

- $11^{\text {th }}$ Conference of the European Sonochemistry Society in La Grande Motte, France 1-5 June 2008 


\section{Conferences and Workshops, presented by collaborators*}

- Stricker,* L., Fernández Rivas, D., Zijlstra, A. G., Gardeniers, J.G.E., Lohse, D. and Prosperetti, A. Ultrasound nucleated bubbles and their sonochemical radical production. $8^{\text {th }}$ International Symposium on Cavitation, Singapore, August 13-16 2012.

- Segers, ${ }^{*}$ T., van Hoeve, W., Fernández Rivas, D., Versluis, M., Acoustic bubble sorting: contrast enrichment by primary radiation forces Lorentz Center. Acoustic Waves for the Control of Microfluidics Flows. April 23-27 2012.

- Rooze, ${ }^{*}$ J., Fernández-Rivas, D., Gardeniers, J.G.E., Rebrov, E., Keurentjes, J.T.F. \& Schouten, J.C. (2010). Hydrodynamic cavitation in a microchannel. Proceedings of the 11th International Conference on Microreaction Technology (IMRET 11), March 8-10 March 2010, Kyoto Japan, (pp. 56-57).

- Zijlstra, ${ }^{*}$ A.G., Fernandez Rivas, D., Versluis, M., Mertens, P.M., Gardeniers, J.G.E., Prosperetti, A., Lohse, D. (2010). Acoustically-driven microbubble pinch-off. Proceedings of $20^{\text {th }}$ International Congress on Acoustics, ICA 2010 23-27 August 2010, Sydney, Australia.

\section{Honors, Awards \& Scientific visits (since 2007)}

- Young Business Award, for the pitch on a business idea for a spin-off named $\mathrm{B} \mu \mathrm{bCLEAN}$, in collaboration with Bram Verhaagen (POF); given at MESA+ Day, Enschede, The Netherlands, September 2012.

- Young Sonochemist Award, given by Japan Society of Sonochemistry, Nagoya, Japan, November 2011.

- Best poster award. Given at MESA+ Day, Enschede, The Netherlands, September 2010.

- School of Physical and Mathematical Sciences (SPMS) Nanyang Technological University Division of Physics and Applied Physics, Singapore 2011.

- Experiments on cavitation with transparent pits and pressure mapping of a microreactor. Prof. C.D. Ohl.

- National Industrial Research Institute of Nagoya AIST, Japan 2011.

- Experiments on the Sonoluminescence and Sonochemiluminescence of sono-microreactors. Dr. K. Yasui and Dr. T. Tuziuti. 
- University of Melbourne, Australia 2011.

- Experiments on the Sonoluminescence and Sonochemiluminescence of sono-microreactors. Prof. M. Ashokkumar and Prof. R. Manasseh.

- von Karman Institute for Fluid Dynamics. Brussels, Belgium. 8-12 October 2007.

- Measurement Techniques in Fluid Dynamics

- Internacional Centre for Theoretical Physics, ICTP, Trieste, Italy.

- Introduction to Nanofluidics, August 2007

\section{Advising}

- Graduate students:

MSc. Henk Klooster. (University of Twente 2012) Currently working.

MSc. Jorge Rodríguez Ramos: Modelación numérica de microreactor bifásico líquido-líquido de canal alargado con reacción química de primer orden. InSTEC 2007.

- Undergraduate Students:

BSc. Joris Betjes: Effects of acoustically induced cavitation erosion on micromachined silicon surfaces. (University of Twente 2012).

BSc. Hylke Donker: On the mechanism of acoustically induced cavitation erosion of brittle materials. (University of Twente 2011).

BSc. Saskia Schildkamp: Efficient sonochemistry using micromachined surfaces. (University of Twente 2010).

Dipl. Eng. Adrián Reyes Cruz: Modelación y estudio experimental del flujo de eslug en micro reactores capilares. (InSTEC 2010). 
Acknowledgements 


\section{Acknowledgements}

It will be difficult to mention all the names (institutions and people) that contributed, or influenced on the finalization of my $\mathrm{PhD}$. To those who made my life more difficult: Thanks for the challenge; in the end I managed with the help of those mentioned here and perhaps some I accidentally missed. I apologize for any omission; it was not on purpose. How could a Cuban end up doing a $\mathrm{PhD}$ in Twente? In the last five years I had to answer this question several times, and it goes more or less like this:

In the summer of 2005, the International Centre for Theoretical Physics (ICTP) in Trieste, Italy, hosted a School on Microfluidics and Prof. Joe Niemela helped me to sneak in into the participants list. There I met Prof. Sreenivasan and Prof. Surya Raghu who later became my MSc. Supervisors. Teaching at the course, there were two professors that drove from the University of Twente (UT): Han Gardeniers and Regina Luttge. I felt such a good connection with both of them that it encouraged me to drop by the UT in December 2005. I was in Europe again thanks to a scientific visit supported by Prof. S. Turek in the University of Dortmund, Germany and with the "unique smuggling assistance" of my good friend Frank Platte (Pancho). That time I got to meet Roald and Kevin while showing me Enschede's "night live" attractions. But I was still not ready to jump in the boat.

After obtaining my MSc. in Cuba, Han and I met again in Algeria in November 2006 while on a conference organized by our friend Dr. Arezki Benfdila. There I had the unique opportunity to choose between two projects and enroll as a $\mathrm{PhD}$ candidate. I was still working for the Instituto Superior de Tecnologías y Ciencias Aplicadas (InSTEC), Havana, Cuba, at the Dpto. de Ingeniería Nuclear with Prof. L. García, Prof. M. Piedra and M.E. Montesinos who appreciated my efforts to open a Microfluidic research line.

So far the story would not differ much from most foreign students in Twente, but the Cuban Ministry of Science Technology and Environment (CITMA), in combination with the whole "stalinist" migration rules existing in Cuba, did not allow me to enroll as a regular candidate, but as part-time employee from August 2007. Initially I was supposed to work six months in Twente and three months in Havana during five years and I was not allowed to travel with my family.

Around that time, the Cuban State Council (OCE) was busy spinning-off a research center with focus on Nanosciences (CEAC) and they were also interested in my progress in the emerging field of microfluidics. The "spoken agreement" was that I could receive authorization to travel with my daughter Gaby and her mother Maylín, within one or two years of the start of my PhD. So I travelled to The Netherlands to start my quest to obtain a $\mathrm{PhD}$.

That agreement vanished when I realized that there was no interest from the Cuban government in moving my family to The Netherlands while I was doing my PhD. I presented my resignation to the then Rector, Prof. Guzmán, in August 2010, 
and became a "half-free cuban". This posed a risky situation and some sort of moral values crisis. As a "half-free cuban" I could travel abroad within certain conditions: to pay the Cuban Government every time I entered and left my own country and never stay in any other country for more than 11 months. Failing to do so would incur in the loss of my properties in Cuba and I would be deprived of all my rights as a Cuban citizen. And all this when my entire life I have defended "leftish" ideals. Unfortunately this situation has not changed, but on the other hand in Twente things went quite well.

When I arrived to Twente, Mesoscale Chemical Systems group (MCS) was in diapers and the Meander building labs were almost empty. Since my project was formulated in collaboration with the Physics of Fluids Group (PoF), Han suggested me to talk to Prof. Michel Versluis, whom I asked for potential collaborators, and available equipment to start doing experiments. Michel introduced me to Valeria Garbin who gave me a tour of the group labs and connected me with Wim, Jeroen, Arjan, Rory, Bram Borkent and Chao. Thanks to them I have got some experiments started and saw how the "system" works.

At the first project committee meeting I met another PhD student from Eindhoven, Joost Rooze, who shared with me part of the frustrations of the first two years as $\mathrm{PhD}$ student. Despite the challenges we succeeded and got some things done!

Eventually I met Prof. Detlef Lohse, with whom I have had interesting discussions during formal meetings, at Lorentz Center in Leiden, or brief ones in front of the coffee machine. With time, new collaborators arrived to the team. Aaldert "enjoyed" as much as I playing around with micromachined surfaces and ultrasonic devices; being your paranymph was a privilege. Laura managed to provide us a solid numerical insight into our experiments and we all had some rough periods getting our "Ulyses" published. At some point, the experiments we were carrying out drew the attention of Prof. Andrea Prosperetti. Without Andrea's guidance, suggestions, and corrections, I would not have acquired most of the knowledge I have on bubbles and their capricious behavior.

Later on, I met the invaluable never-resting collaborator and good friend Bram Verhaagen. We managed to make experiments combining our ambitions, knowledge, and projects with a very good output. Now it seems we will become CEO and CTO from a successful spin-off called $\mathrm{B} \mu \mathrm{BCLEAN}$. Together with other students, such as Wilco, we managed to expand even more the MCS-PoF ties. We have looked at bubbles cleaning inside teeth root canal models and got involved with other good fellows: Ricardo, Guillaume, Tim and Elisabeth; good fun in the lab guys! I know you all wanted me to write about my "bubble analogies", but it would be too long and hard to explain. Sander, thanks for sharing the enthusiasm with coloring my crazy bubbles sketches and cover design! The good collaboration established with PoF would have not been possible without the always efficient assistance of Joanita, 
Gert-Wim, Martin and Bas.

On the MCS group I had the chance to learn and interact with good fellows like: Jacob, Dawid, Anil, Nikolai (paranymph fellow for Liza), Maciej (paranymph fellow for Niko), Arturo, Selm, Mattia, Hoon, Engin, Roland, Sertan, Bilge, Liza (my favorite enemy), and many visitors such as Takayuki, Reyes,... impossible to get all the names here! Jaqueline, bedankt voor de dagelijkse Nederlands oefening. Stefan (fellow "Dudeism" priest) no idea what would have been of myself without your support in the Cleanroom and funny endless jokes, and active karting enthusiasm (I almost forgot to thank $3 \mathrm{M}$ for the adhesive tape!!). Taming students such as: Saskia, Hylke, Joris, Henk and some in POF was always an interesting experience. The help from Bauke Visser from the HR department was decisive in the many battles with bureaucracy.

Han, thanks a lot for allowing me the possibility not only to plan experiments with you, but for so many interesting political, social, and more important, personal debates. Your support and Ingrid's is something I will always remember.

In five years I have interacted with many collaborators, some in Twente and some really far away: Ton (OPM), thanks for joining the crew on the erosion study. Severine (BIOS), thanks a lot for your support and sharing our dream-experiments. DirkJan (OS), thanks for trying hard to see some spectra in the dark. Pino (TST) was always very supportive with "urgent requests" and Gerald (WA) tried with lasers to obtain weird designs for me. Adriana (OPM), gracias por la buena energía e intentos con el microscopio a última hora. Dirk (NBP), thanks for the several inductions to your lab for me and other colleagues. The MESA+ staff was always very helpful; specially the melodious Mark and his fantastic SEM images. A bit farther in Eindhoven were Bob and Ramon; with some more time we would have achieved what we wanted. Across the border in Belgium, XiuMei is still working in a long-standing collaboration; I am sure success is around the corner!

Working with bubbles allowed me to interact with awesome people living as far as Australia. Thanks to Ashok who welcomed me in his lab I got to meet Franz Grieser, Tom, Daria, Sandra, Adam, all in Melbourne Uni. In Swinburne Uni, Richard Manasseh allowed me to "cut the inauguration ribbon" of his brand new lab, I hope to go back and do more work together. Working in the dark in Japan with Yasui-san, Tuzituti-san and Tom was an incredible "illuminating" experience. Tom, without you mate Australia and Japan would have not been the same! In Singapore, Claus and his team, specially Tandiono and Roberto welcomed me with a "hot" environment temperature. Thanks a lot to the whole gang working in Nangyang!

De la Madre Patria, España he tenido el placer de colaborar con Pedro: un millón de gracias por todo. Lamentablemente nos abandonó inesperadamente Jose García, muy buenas ideas se quedaron en el tintero. En Cuba intentamos hacer algo Ulises, Katia y yo, pero lamentablemente la analítica no nos acompañó. Tengo muy buenos 
recuerdos y extraño los debates, sesiones de té y preguntas a veces tontas que les hice a mis amigos del InSTEC: Alfo, Franklin, Amor y Rolo.

I cannot leave out a bunch of the very good friends that have accompanied me or supported me at any time and from any part of the world. Tante grazie a Suzie, from Trieste, who in 2005 gave me the guitar that is still "partying" with me. In Australia, I would have been bored to death if it were not for my best Irish friend Clodagh, "steampunk" Dave, Lily and many other folks. Back in Enschede, walking on a saturday's market it is not hard to find colleagues from work in the city center. Nevertheless, there are people that I found more often, whether by living, eating, dancing, arguing, etc. with them: Roland e Iza, Wojtek, Dagmara, Burak, Gerhard from Macandra, the "winter-movie-evening-club" with Dawid, Kasia, Piotr and Steffie. Marga en Jet, ik heb met jullie een leuke tijd op vele Nederlandse lessen gehad. Essly, my first Couch Surfing experience, bedankt voor alles!!

I had the privilege to have some "multi-cultural-families": Verdoold's family and specially my brother Vincent and wife Jane. Mi familia Cubana-Holandesa Paul y Nancy, siempre apoyándome en los momentos más duros, comidas, compras, chismes, y buena música. En Alkmaar la familia Arubeña-Colombiana-Cubana con Otto, Rosy e hijos ha sido siempre un hogar y refugio.

La Comunidad Latina de Enschede ha crecido con los años y el número de fiestas ha incrementado de una manera proporcional e incommesurable (no me estoy quejando!). Haber formado parte de la primera generación de LAVoz fue y es un privilegio (aunque miembro fugitivo y rebelde). Me traen siempre los mejores recuerdos al pensar en las bromas de Daniel Chehata (La Eminencia), las veladas en tantos lugares en compañía de Nayeli, Eduardo, Jealemy, Andrea (presidenta), Andreea (bailarina), Olga, Michelle, Nazely, Diana, Enrique, Juan, Diru y su Nicolás; el pequeño Emilio y sus padres Rafael y Mariela, Daniela, Ignacio y sus diablillos Mateo y Santiago. Sandra con sus empanadas y comida criolla me teletransportaron varias veces a Cuba con su sabor criollo y caribeño sentado en Carlina's. Infinitas gracias a mi hermano de tantas andanzas Julián; recuerdo el honor que representó atender a los padres Martínez-Mercado y a su "Juliancito" en La Habana.

Music and art have also been an important part of these last five years, especially since the foundation of two "famous bands": Casual Mango and Chilangos Habaneros. Each band has had a particular "cave-operational base" owned by Willem @San Remo and Michael @Rico Latino respectively. Bedankt voor de samenwerking! Casual Mango was a "casual" band, that as its name indicates, just happened as Oscar and Bob were jamming on the CMLF (Canadian-Mexican Liberation Front). Around the same time, the always creative couple Oscar and Daniela, Julian ("chilangos") and myself (the "habanero") found out that people could dance and have fun with the music we played. In a short period of time the Chilangos Habaneros grew in number and many good friends played along and some continued their way: Bob, Ka- 
sia, Alvaro, Pawel, Marine, Edith, Carla, Mateo, Jorge (y el apoyo de la Sra. Pana, aka Mayte), Anne, Juan Carlos, Diego, Effie, Massi, Maurizio (Papaurizzio, aka Grande Mauro). Gracias a todos por darle los grados a "El Comandante".

A special thanks to my paranymphs Stefan and Oscar (paranimfo en castellano? :D). Thanks for standing by my side on such a remarkable date and ceremony.

I know that paper can hold many things in written form, but is almost impossible to use plain words to express the way I have carried in my mind and heart all my family - those physically gone, and those inspiring me to keep up my fighting instincts. In any case, here is an attempt in Spanish:

Mi mamá viene por supuesto en primer lugar pues sin ella no podría haber llegado (antes de tiempo) a donde estoy. Mis hermanas Claudia(s), mi prima-más-quehermana Wendy, todos los primos y tíos; es siempre reconfortante saber que están, donde sea que estén, políticamente de acuerdo o no. El "primo" Joel y Leanne me ayudaron a desconectar en Cangurolandia; Gracias por la fiestona!

Dos mujeres de suma importancia en mi pasado y que sin dudas siguen conmigo en mi corazón: Mama y Tia, tantas cosas que aprendí y aun así me quedé añorando y necesitando tantas otras. Sin Maylín, su apoyo y amor incondicionales y lo mejor que nos ha pasado y por lo que aún batallamos: Gaby, nada serían mis logros.

And then this is the end of this "adventure"... let "life" bring more problems to solve!

David 
About the author... 
David does not like to write or talk in third person...

Not only it seems weird but some people were accused of insanity for doing that in the past.

I was born in Havana, Cuba in 1981 and since an early age I used to fix my toys and those my friends brought with the hope that I could make them work again - unfortunately I was not always successful.

In the high-school period I managed to combine my studies with learning how to play the guitar and produce vocal sounds resembling what some people call "singing".

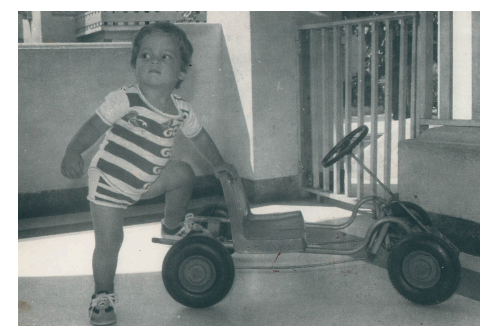

On top of that, due to electricity power-cuts after the communist block collapse, there was no more alternative than to play in front of the neighbors during endless warm tropical nights, which somehow helped me to overcome public fear.

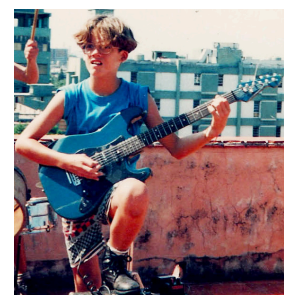

When faced to make a decision on whether to go to music school (and keep playing with the bands I was active with) or to highschool and pursue a "professional" life, I chose to keep music by my side and somehow ended up studying Nuclear and Power Engineering.

The real reason on why that career and not another of the several options I cherished (medicine, geology, physics, etc.), is too complex to type it here; but it turned out to be a great experience and I don't regret a single moment of it.

When I entered the compulsory military service I could have no idea that an event that changed my life forever was coming my way: Gaby was born in 2000. Becoming a young father without the help of family and friends is impossible, so the least I could do in return was to study and work as hard as I could to "honor" so much collective effort.

While working in an International Atomic Energy Agency project, (still as an undergrad student) with unique scientists, professors and friends I started walking in the "Colorful Fluid Dynamics" path. This path lead me to collaborations with colleagues in Europe (bypassing sometimes political regulations) and eventually I detached from my "radioactive past".
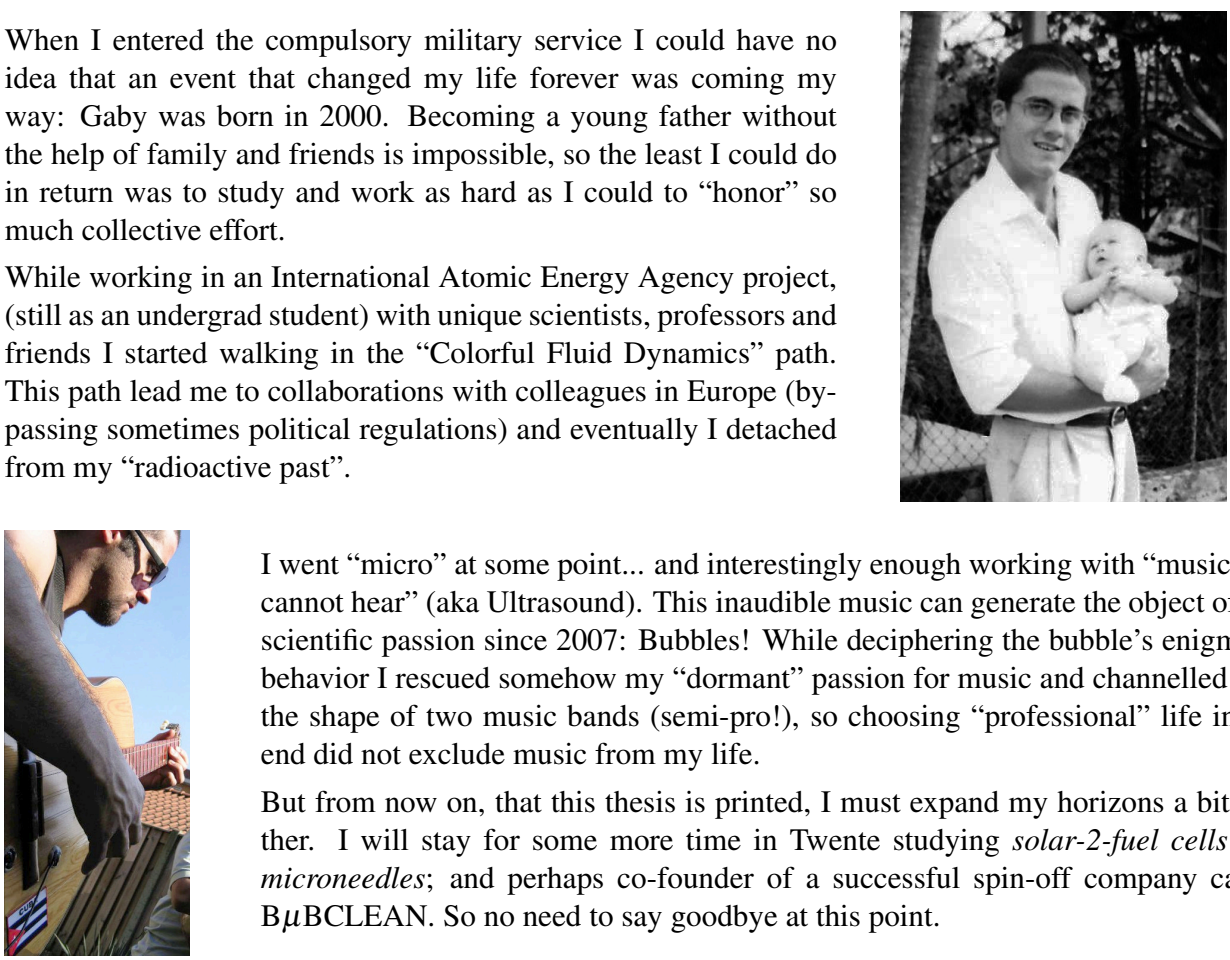

I went "micro" at some point... and interestingly enough working with "music you cannot hear" (aka Ultrasound). This inaudible music can generate the object of my scientific passion since 2007: Bubbles! While deciphering the bubble's enigmatic behavior I rescued somehow my "dormant" passion for music and channelled it in the shape of two music bands (semi-pro!), so choosing "professional" life in the end did not exclude music from my life.

But from now on, that this thesis is printed, I must expand my horizons a bit further. I will stay for some more time in Twente studying solar-2-fuel cells and microneedles; and perhaps co-founder of a successful spin-off company called $\mathrm{B} \mu \mathrm{BCLEAN}$. So no need to say goodbye at this point. 